Florida International University

FIU Digital Commons

$11-16-2020$

\title{
Racial Microaggressions of Indigenous Undergraduate Students in an Ecuadorian Higher Education Institution
}

Adriana Cundar Ruano

acund003@fiu.edu

Follow this and additional works at: https://digitalcommons.fiu.edu/etd

Part of the Education Commons

\section{Recommended Citation}

Cundar Ruano, Adriana, "Racial Microaggressions of Indigenous Undergraduate Students in an Ecuadorian Higher Education Institution" (2020). FIU Electronic Theses and Dissertations. 4562. https://digitalcommons.fiu.edu/etd/4562

This work is brought to you for free and open access by the University Graduate School at FIU Digital Commons. It has been accepted for inclusion in FIU Electronic Theses and Dissertations by an authorized administrator of FIU Digital Commons. For more information, please contact dcc@fiu.edu. 
FLORIDA INTERNATIONAL UNIVERSITY

Miami, Florida

\section{RACIAL MICROAGGRESSIONS OF INDIGENOUS UNDERGRADUATE STUDENTS IN AN ECUADORIAN HIGHER EDUCATION INSTITUTION}

A dissertation submitted in partial fulfillment of the

requirements for the degree of

DOCTOR OF PHILOSOPHY

in

TEACHING AND LEARNING

by

Adriana Cundar Ruano

2020 
To: Dean Michael R. Heithaus

College of Arts, Sciences and Education

This dissertation, written by Adriana Cundar Ruano, and entitled Racial Microaggressions of Indigenous Undergraduate Students in an Ecuadorian Higher Education Institution, having been approved in respect to style and intellectual content, is referred to you for judgment.

We have read this dissertation and recommend that it be approved.

$\begin{array}{r}\hline \text { Sarah Mathews } \\ \hline \text { Ana Luszczynska } \\ \hline \text { Aixa Pérez-Prado } \\ \hline \text { Eric Dwyer, Major Professor }\end{array}$

Date of Defense: November 9, 2020

The dissertation of Adriana Cundar Ruano is approved.

Dean Michael R. Heithaus

College of Arts, Sciences and Education

Andres G. Gil

Vice President for Research and Economic Development and Dean of the University Graduate School

Florida International University, 2020 
C Copyright 2020 by Adriana Cundar Ruano

All rights reserved. 


\section{DEDICATION}

In sad but truly loving memory of Dr. Joane Sanders-Reio who departed from this world in December 2019.

The first time I met Dr. Sanders-Reio was in Fall 2018 whilst a student at Florida International University. I immediately recognized her compassion and empathy. She was always ready to provide support to her students and she did it with a great sense of love and affection. In our conversations, she generously shared her wisdom that went much beyond the course content. She kindly worked as member of my Committee to which she provided guidance, encouragement and hope. My respect and appreciation to an erudite academic and an extraordinary human being.

I humbly dedicate this achievement to God in whose hands I put this pursuit. My faith gave the strength and determination to overcome the obstacles I have found along this stage in my life. My Lord's love and compassion worked countless miracles every single day.

A special feeling of gratitude to my loving children Andres and Adriana whose endless love, support, and offering have been the pillars that pushed for tenacity and resolution. I dedicate this degree to both of you my guardian angels on earth. You will always be the most precious flowers in my garden. You are and will continue to be my greatest achievement.

I am thankful for the love and companionship of my Mother who always believed in me. Thanks to you Mamita Linda for envisioning in education, the power to change my life. Despite you did not have the opportunity to study yourself, you struggled to provide me with one. Your life has been my inspiration. Because I saw you working in 
arduous conditions, mistreated, and invisible but strong and determined; I embarked in continuing my studies to return your sacrifice. This work is my humble tribute to your suffering.

Finally, I want to leave a note of appreciation to my beloved friends for being there for me with your wise advice and encouraging words. Thank you Elizabeth, Laura, Perla, Fanny, Ligia, Jenny, Holly, Roy. 


\section{ACKNOWLEDGMENTS}

First and foremost, I want to sincerely acknowledge and thank my major professor Dr. Eric Dwyer and my dissertation committee, including Dr. Ana Luszczynska, Dr. Sarah Mathews and Dr. Aixa Pérez-Prado for their expertise and precious time to guide me along this entire process. I am forever indebted to my committee. I will never pay you back for your patience and encouraging words that inspired me to achieve this lifetime dream. I am very thankful with all of you for being role models and for always making sure I am in the right direction. This dissertation reflects your long hours of dedication and patience.

I want to express my special recognition to Dr. Dwyer for his leadership and for trusting me. This paper responds to your interest in knowing what personal and professional happenings resulted in me leaving my comfort zone in my home country to embarking in this extraordinary learning process. Your deep questions and reflections sparked my inner self to give voice to the voiceless.

My sincere gratefulness to my twelve Indigenous students who participated in this study. Thanks for sharing with me your stories of oppression and courage. I hope I was able to condense your struggles and dreams in this modest work. That God blesses your deeds and gives you the strength to continue your battle.

No least of all, my note of appreciation to Florida International University where I felt welcomed and respected as a human being. Thanks for providing with all the necessary tools to succeed. 


\title{
ABSTRACT OF THE DISSERTATION \\ RACIAL MICROAGGRESSIONS OF INDIGENOUS UNDERGRADUATE STUDENTS IN AN ECUADORIAN HIGHER EDUCATION INSTITUTION
}

by

\author{
Adriana Cundar Ruano \\ Florida International University, 2020 \\ Miami, Florida \\ Professor Eric Dwyer, Major Professor
}

\begin{abstract}
Although subtle, racial microaggressions have an impact on lives of people who endure hostilities by virtue of their race or ethnicity (Solórzano et al., 2000; Sue et al., 2007). Research on racial discrimination indicates that college campuses can be threatening environments especially for underrepresented students who constantly face elusive hostilities in their interactions with teachers, peers, and administrative (Allen, 2010; Helm, 2013; Nadal et al., 2014, Reid, 2017). The current mixed-methods study examined the most commonly experienced types of microaggressions affecting Indigenous undergraduate students while pursuing a bachelor's degree in a public higher education institution in Ecuador, as well as perceived relationships between microaggressions and students' self-esteem and self-efficacy. The present study also analyzed experiences of Indigenous students in regard to their responses and coping mechanisms for racial hostilities.

A sequential transformative mixed methods design was used to survey and interview participants. In the quantitative component, three surveys-Nadal's Racial and Ethnic Nadal (2011) Microaggressions Scale (REMS), Rosenberg's Self-Esteem Scale
\end{abstract}


(SES) and Schwarzer and Jerusalem's General Self-Efficacy Scale (GSE) — were administered to 112 Indigenous students. In the qualitative component, a semistructured interview was conducted with 12 participants. A Pearson Correlation coefficient was ascertained to assess the relationship between microaggressions and Indigenous students while emotion coding was utilized to capture themes from students' accounts. Findings suggested that Indigenous students experience six principal types of microaggressions contained in the REMS, with assumptions of inferiority and microinvalidations named most commonly by participants. Results showed negative associations between three components of the REMS and students' self-esteem, while a negative correlation was observed for five of the six components of the REMS and self-efficacy. Students' lived narratives revealed the profound manifestations of oppression they endure in their academic environment. Responses to microaggressions were described by participants as those of resilience, pride, and affirmation of identity. Students' accounts revealed that their main coping mechanism to hostilities refers prominently to family support as they pursue academic endeavors. A key recommendation coming from the study suggests creating space where both Indigenous students and university officials can learn about and understand how microaggressions can affect on-campus experiences. 


\section{TABLE OF CONTENTS}

CHAPTER

PAGE

PREFACE 1

DEFINITION OF KEY TERMS …...................................................................

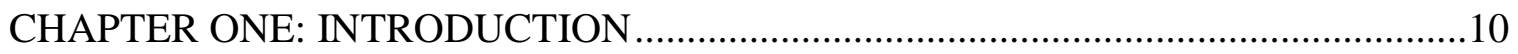

Microaggressions ............................................................................... 10

Microaggressions and Self-esteem ................................................14

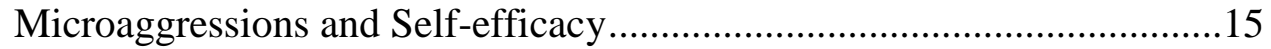

Reacting and Coping with Microaggressions ......................................17

Indigenous Populations and Microaggressions in Ecuador .....................20

Purpose of the Study ..............................................................................22

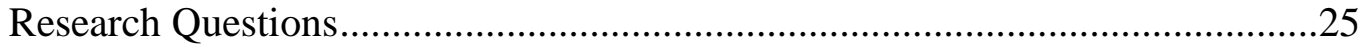

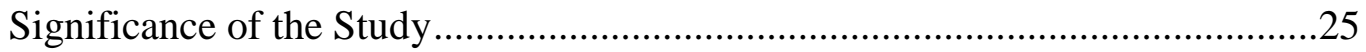

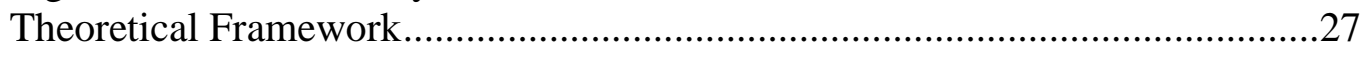

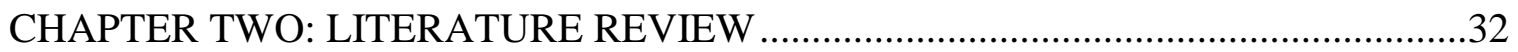

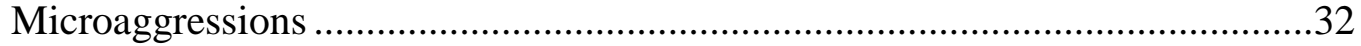

Definitions and Types of Microaggressions ............................................32

Associations between Microaggressions and Mental Well-being ..............38

Microaggressions and Self-esteem ...................................................43

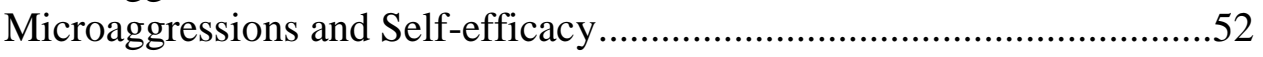

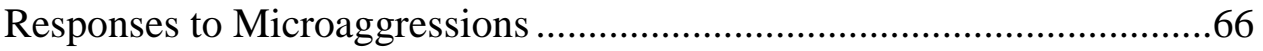

Coping with Microaggressions .....................................................69

Indigenous Populations, Schooling, and Microaggressions in the

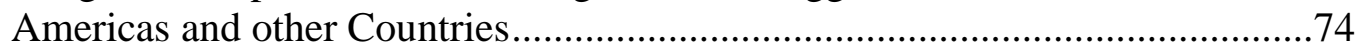

Ecuadorian Context and Indigenous Education ............................................8

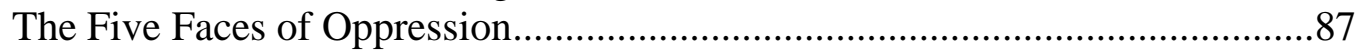

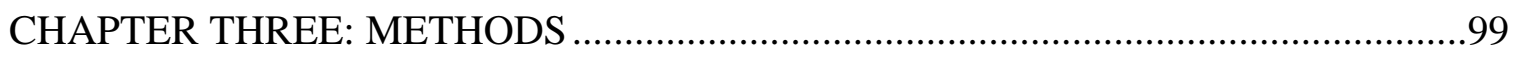

Comparing Positivist and Naturalistic Approaches .....................................101

The Mixed Methods Research Paradigm .......................................................103

Rationale for Sequential Transformative Approach ......................................108

Quantitative Component .......................................................................109

Study Participants and Inclusion Criteria .........................................110

Quantitative Data Collection Procedures ...........................................111

Quantitative Data Analysis ..............................................................113

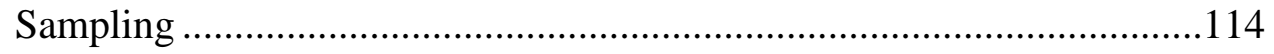


Research Question One. What are the Most/Least Common Types of Racial Microaggressions that Indigenous Students Experience while Pursuing a degree in a Public Higher Education Institution? .116

Definition and Examples of Constructs in the REMSs Survey ...118

Possible Problems with REMS and Ethical Issues .....................121

Research Question Two. What is the Association between Microaggressions and Indigenous Students' Self-esteem.

Research Question Three. What is the Association between Microaggressions and Indigenous Students' Self-efficacy? ..................125

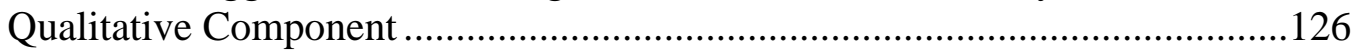

Qualitative Sampling and Participants..............................................129

Qualitative Component Rigor ..........................................................130

Qualitative Data Analysis .................................................................131

Research Question Four. How do Indigenous Students Experience

Racial Microaggressions while Pursuing a Degree in a Public

Higher Education Institution on Issues of Responses to

Microaggressions, and Mechanisms they use to cope with

Microaggressions

Themes Addressed in the Qualitative Interview ........................134

Chapter Summary

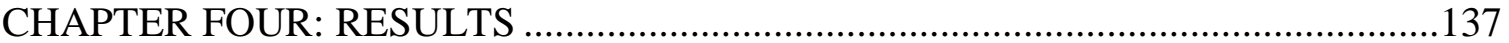

Component One: Quantitative Study Results ..............................................138

Participants' Demographic Information and Descriptions ....................138

Research Question One. What are the Most/Least Common Types

of Racial Microaggressions that Indigenous Students Experience

while Pursuing a Degree in a Public Higher Education Institution? .......142

REMS Component One: Assumptions of Inferiority...................145

REMS Component Two: Second-Class Citizenry and

Assumptions of Criminality ...................................................146

REMS Component Three: Microinvalidations ..........................147

REMS Component Four: Exoticization and Assumptions of

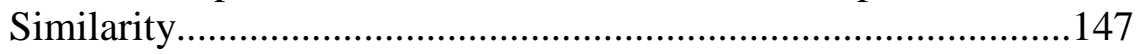

REMS Component Five: Environmental Microaggressions........148

REMS Component Six: Workplace and School

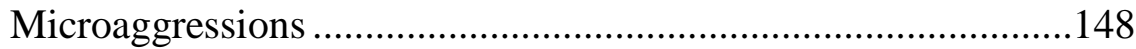

Research Question Two. What is the Association between

Microaggressions and Indigenous Students' Self-esteem.

Research Question Three. What is the Association between

Microaggressions and Indigenous Students' Self-efficacy?.... 
Component Two Qualitative Study Results...................................................152

Participant's Introduction and Pseudonyms..........................................152

Experiencing Microaggressions............................................................156

Assumptions of Inferiority ........................................................156

Second-Class Citizenry and Assumptions of Criminality ...........159

Microinvalidations ........................................................................160

Exoticization and Assumptions of Similarity ..............................161

Environmental Microaggressions ................................................163

Workplace and School Microaggressions.......................................164

Research Question Four. How do Indigenous Students Experience

Racial Microaggressions while Pursuing a Degree in a Public

Higher Education Institution on Issues of Responses to

Microaggressions, and Mechanisms they use to Cope with

Microaggressions?

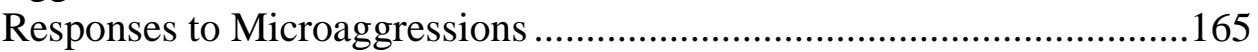

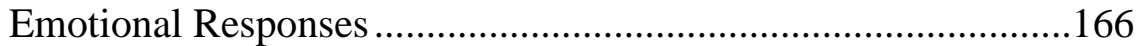

Internal Struggles …………………………………....166

Positive Emotions ............................................................170

Negative Emotions ..........................................................173

Behavioral Responses .....................................................................176

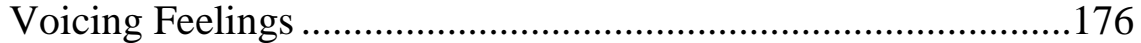

Mechanisms Students use to Cope with Microaggressions .......................178

Interpersonal Support.................................................................178

Parental and Immediate Family Support..........................179

Friends' Support..........................................................180

Stress Relief Activities.............................................................180

Easing up Anxiety ........................................................181

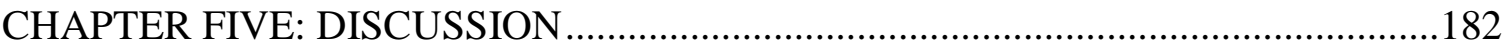

Occurrence of Racial Microaggressions .......................................................183

Microaggressions and Self-esteem ………………….................................184

Microaggressions and Self-efficacy............................................................186

Microaggressions Experiences...................................................................190

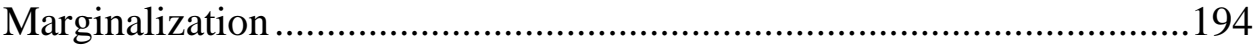

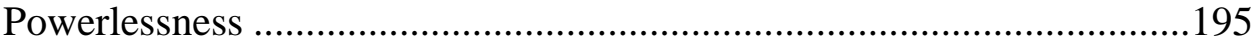

Cultural Imperialism ........................................................................196

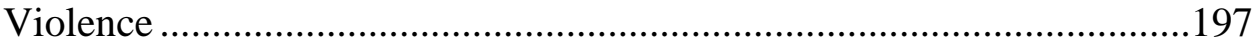

Responses to Microaggressions and Coping Mechanisms ...............................197

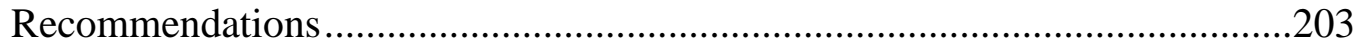

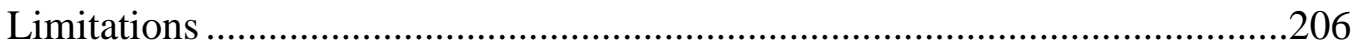

Implications for Further Research ............................................................208

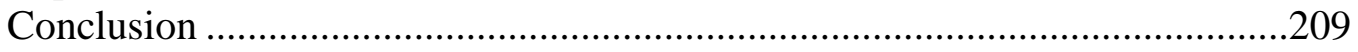


REFERENCES

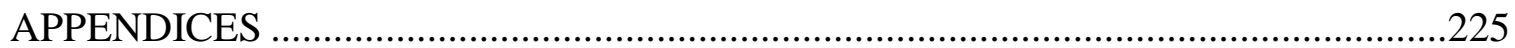

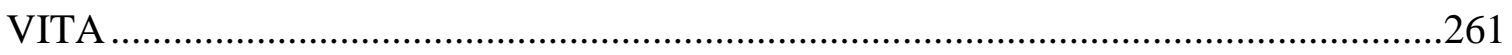




\section{LIST OF TABLES}

TABLE

PAGE

1. Students Self-Identified as Indigenous by College Updated to Fall 2019 115

2. Racial and Ethnic Microaggressions Scale Frequency of Event 118

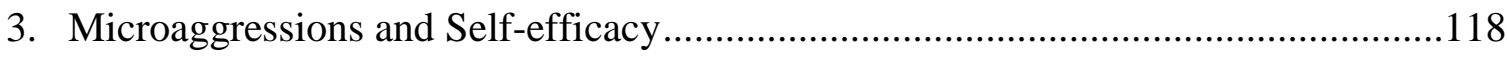

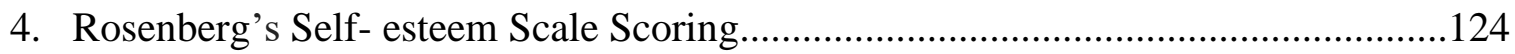

5. Schwartzer and Jerusalem's General Self-Efficacy Scale Scoring ..........................126

6. Racial Microaggressions Semistructured Interview Domains and Leading Statements

7. Summary of Self-Reported Demographic Information

8. Occurrence of Microaggressions. Means and Standard Deviations of REMS

Components

9. Students' Perceptions of Occurrence of Microaggressions. Means and Standard Deviations Summary of Components

10. REMS Correlations for Self-esteem

11. REMS Correlations for Self-efficacy.

12. Summary of Themes and Subthemes for Emotional Responses to Racial Microaggressions

13. Summary of Themes and Subthemes for Behavioral Responses to Racial Microaggressions

14. Summary of Themes and Subthemes for Coping Mechanisms .178 


\section{PREFACE}

"Indigenous students experience schooling, but schooling does not experience them."

My paper responds to my need to give voice to those who have remained in silence for centuries, those whose presence is uncomfortable, those who despite of having suffered all kinds of oppression remain in their struggle to thrive. My paper is a tribute to Indigenous students in all levels but especially to those in higher education.

Talking about racism and oppression is not an easy task, especially in a society that denies the existence of prejudice and segregation. The journey that has taken me to work on unveiling issues related to racism started long ago when I was a child and has continued developing to the present time. My stand is that racial microaggressions have their origin at school, when teachers misunderstand and undervalue students who they believe are less capable by virtue of their race, ethnicity, or social status. It is at school where microaggressions initiate until they permeate society as a whole. Having experienced microaggressions at school and having lived oppression at some point in my life, legitimate and strengthen my eagerness to analyze how racial hostilities impact Indigenous students' journeys in college. My hope is that my study will illuminate how microaggressions are lived in Ecuadorian context. The present study is not rooted in my experience as a student but as a teacher where I have witnessed the struggles of Indigenous students who, despite their efforts, remain unnoticed and microaggressed in educational institutions.

I remember vividly my first encounter with the teaching profession in a school whose population was made up of a majority of Indigenous children. As an acute observer, the school became my boundless s1ource of inquiry. I started noticing events 
that seemed to others ordinary, if not trivial. I noted, for instance, that some Indigenous children came to school, holding the hand of their mother or grandmother. The fact that mothers left their work in the fields for a while to take their children to school had a profound meaning to me. My thoughts were that, despite Indigenous families' limitations in providing an education to their children, these families were convinced that education was the only legacy that they could offer their children to ensure them a more promising future. Sadly, for most Indigenous students, their path to school is plagued by stereotypes and microaggressions.

From my perspective, Indigenous children were incredibly smart and skillful. However, few educators would be able to understand their capabilities. As early as when Indigenous children walked in the classroom, their black coal eyes seemed searching on the gray school walls, the colors of the flowers, the texture of the soil, and the fragrance of the crops in their fields. In a clear manifestation of unintentional microaggression, teachers would say these children were easily distracted, unable to focus, and unable to learn as their peers did. Teachers did not realize that behind the apparent distraction, children would struggle to connect the way they conceived the world with the prescribed reality the school imposed on them. Thus, Indigenous children's efforts to re-adjust to this "new" world were certainly overlooked by teachers. Apart from the lack of concentration, Indigenous children were also described as being rough and unskilled. Children had a hard time when working with their fine motor skills. It would take them very long hours to master school tasks such as cursive writing and drawing. Their hands would certainly not adapt to the use of the slender pencil as they did with the tools they used to cultivate the fields. Therefore, children were designated as "inadequate" at some 
school assignments. However, Indigenous children would exhibit exceptional abilities when using their gross motor skills: They excelled at running, playing, and climbing trees. They would also use their gross motor skills to create all sorts of items with few resources. Unfortunately, students' apparent inadequacy for scholarly work was consistently highlighted. As a result, teachers would label Indigenous children as not being academically inclined. Children were microassaulted when they were assigned "deficiencies" for the fact of being Indigenous, microinsulted when receiving derogatory messages such as "slow learners." and microinvalidated when their thoughts, feelings, and experiences were not considered as valid in the educational environment.

From my early experience as a teacher, I witnessed how Indigenous students were stereotyped with a variety of unfavorable labels that did not acknowledge the countless potential these students brought to school. My naive thinking was that as Indigenous students progressed in the school system, these perceptions as their being inadequate to learning, lazy, or ill-mannered would fade away. Sadly, when stereotypes are reinforced often enough, they become ingrained in all realms affecting how people view Indigenous people, how people view themselves, and what they think they understand about Indigenous cultures. Preconceptions are harmful because once the very few Indigenous students reach college level, stereotypes that generate microaggressions have already permeated to all the spheres they transit, including of course the institutions where they pursue a degree.

To my surprise, as soon as I transitioned to teaching at university level, I witnessed no indication that these microaggressions shifted much over the years. I started hearing similar comments from fellow colleagues who expressed their frustration when 
teaching classes where Indigenous students were present. It was common to hear teachers saying that they could not allot more time to the special needs of Indigenous students at the cost of others. They would also say that once in the university, some Indigenous students exhibit difficulties in the use of Spanish, both in oral and written form, resulting from the interference of their Quichua. Criteria for oral language was that Quichua pronunciation hindered the "correct" use of Spanish, resulting in awkward utterances. Lack of vocabulary as well as inability to express clear complete thoughts were also some of the "deficiencies" Indigenous students showed at the university level. In regard to writing, teachers remarked on students' limitations to produce deep, coherent, and cohesive information. Faculty would conclude, at this point of the students' learning trajectory, that it was too late to address their shortcomings.

Once Indigenous students reach college, perceptions are that they are slow learners who do not contribute to the improvement of their own education and whose participation in class is not what is expected of a college student. The identified weaknesses are of course reflected in their alleged "low performance, low achievement, and ultimately low graduation rates." At college, Indigenous students are also invisibilized as their presence in crucial college events is minimal, practically nonexistent, except for festivals where their culture is depicted as exotic. In general, Indigenous students' experience at college is replete with all kind of hostilities by virtue of their group membership in casual but recurrent ways that undermine their energy.

Unfortunately, being immersed in the system for a long time is likely to result in making teachers insensitive to students' challenges. It is common then that teachers and authorities see the problems around them but do not take any action in making a 
difference. Yet more everyday inequities appear to be normal and therefore accepted. This ordinariness of injustice against disadvantaged students is what I believe Macedo (2000) describes as "students being present and yet not visible, visible and yet not present” (p. 11). Macedo's assumption condemns Indigenous students from the very beginning of their schooling to remain at the periphery, limiting themselves to occupy marginal or secondary positions that let them survive in the system until they obtain their degree. Over the course of years, Indigenous students learn to remain silent and unnoticed, they transit the system accepting how it works and functions to maintain the already established hierarchical levels. Schooling instills in them the position they are supposed to conform to society.

Despite their invisibility in school, Indigenous students who bravely survive to obtain a degree get insights about the system's dynamics and logic. They incorporate all kind of messages from their experience as students and citizens. They learn to resist and this resistance allows them to penetrate and to some extent assimilate schooling. However, penetration takes only one direction since schools do not attempt to understand, facilitate, sustain, or promote the holistic development of Indigenous students. The statement made at the beginning of the chapter — in the sense that Indigenous students' experience schooling but schooling does not experience them—seems valid. Schools where Indigenous students navigate neglect this population in terms of understanding who they are - their culture, background, and history. Often, institutions look at the tip of the iceberg of the experience Indigenous students have at school. The surface is portrayed in festivals and traditional celebrations that makes Indigenous people more a costume than a culture. However, their essence and what their culture brings to college remains 
unexplored in terms of understanding Indigenous students' ways of thinking, learning, experiencing, and being targets of racialized hostilities at school. Sadly, their contributions and what they bring to education are yet to be discovered and discussed in their complexity. In the same manner, the different faces of their oppression remain invisible in their institutions.

By conducting my study, I have aspired to adopt a position of agency, both from an educational and ethical perspective. I want to take the steps to create awareness that results in schools experiencing lives of Indigenous students—an experience that hopefully results in decreasing microaggressive behaviors. I strongly believe that there is still a lot to do to make education a real opportunity for Indigenous people to succeed and break the oblivious oppression they have suffered throughout the years. 


\section{DEFINITION OF KEY TERMS}

Blancos: According to the 2020 Census, $6.1 \%$ of Ecuadorian population selfidentify as White. This population refers to those who are of predominantly European descent. However, defining ethnic groups in Ecuador and particularly Blancos is difficult since people tend to switch their ethnic affiliation according to numerous factors such as social status and financial standing. Some people self-identify as Blancos despite they know that there is a fusion of Indigenous genes in their heritage. People self-identify as Blancos because it gives them power, prestige, and legitimacy. My study refers as Blancos or White Mestizos to the Blanco population.

Illiteracy: Merriam-Webster (n.d) defines illiteracy as the quality or state of being illiterate especially to the inability to read and write. In my paper, the term illiterate goes beyond this definition to refer to people who despite being able to read and write, are unable to properly perform tasks where literacy is required for their own benefit and their community development.

Indigenous: It is difficult to devise a universal definition for the term Indigenous because of the different cultural manifestations the term implies. For the purpose of my paper, Indigenous refers to those individuals who descend from former native cultures inhabiting Ecuador before the Spanish conquest. As a group, Indigenous people share cultural practices, self-identity, language, sociocultural affiliations, and the perception of nature as the genesis of life. An approximate $7 \%$ of the population self identifies as Indigenous in Ecuador. 
Mestizaje: The racial mixing between the Spanish and the people who inhabited the land of Ecuador before the Spanish conquest. My study conceives Mestizaje not limited to the mixing of races but to the mixing of social and cultural attributes. In my paper, Mestizaje also refers to the acculturation of Indigenous people into the dominant culture (Mestizo) to fit the demands of society.

Mestizo: In Ecuador, Mestizos are $72 \%$ of the population (Instituto Nacional de Estadística y Censos [INEC], 2016). In my paper, the definition for Mestizo is a person descendant of a Spanish and Indigenous mixture. From the social and political standpoint, being a Mestizo in Ecuador implies the increase of social opportunities and the lessening of economic difficulties that society imposes to Indigenous people because of their ethnicity. In many cases, individuals who choose to self-identify as Mestizo, do so to avoid discrimination.

Microaggressions: Brief and commonplace daily verbal, nonverbal, behavioral, or environmental slights, snubs, or insults, whether intentional or unintentional, that communicate hostile, derogatory, or negative messages to target persons defined solely upon their marginalized group membership (Sue, et al., 2007).

Self-Efficacy: Bandura (1977) referred to self-efficacy as the personal beliefs about one's capabilities to learn or perform actions at designated levels.

Self-Esteem: Self-Esteem is defined as the individual's opinion or a particular view of one's own self-worth, self-respect and self-confidence and the extent to which the individual considers positive or negative opinions about self (Sedikides \& Gress, 2003). 
Violence: Dimension of oppression that implies a systemic social practice where oppressed people are subjected to unjust treatment for the simple fact of being members of a group that has been assigned as underclass (Young, 2004).

Whiteness: My study conceives whiteness as the position adopted by Mestizos and White Mestizos to establish boundaries between them and other ethnic groups such as Indigenous. These boundaries are established in reference to socioeconomic status, color of skin, and the position people occupy in a group. Relations of power are determined in accordance with how White an individual is considered. 


\section{CHAPTER ONE \\ INTRODUCTION}

My mixed methods study advocates for the improvement of Ecuadorian higher education by exploring microaggressions. The study aims to examine the types of microaggressions affecting Indigenous undergraduate students while pursuing a Bachelor's degree ${ }^{1}$ and the relationships these microaggressions have on their selfefficacy and self-esteem. It also aims to explain the experience of Indigenous students with respect to how they respond to and cope with microaggressions.

Chapter One presents an overview about microaggressions, self-esteem, selfefficacy, and mechanisms people adopt to respond to and cope with racial incidents. The chapter then explores the occurrence of microaggressions in Ecuadorian context. The elements described at the beginning of the chapter lead to the identification of the purpose of the study and the research questions. Finally, the chapter includes a summary of five faces of oppression that shall serve as the lens through which microaggressions in Ecuador will be analyzed.

\section{Microaggressions}

Educational systems are indicative not only of social mobility but also of antagonism between groups divided by social and racial differences (Iannelli, 2013; Reay \& Vincent, 2013). Schooling may act as the critical mechanism through which the system maintains, reproduces, and naturalizes inequalities (Bourdieu \& Passeron, 1977). The assertion made by the authors seems accurate for Indigenous people in Ecuador because,

\footnotetext{
${ }^{1}$ Degree awarded by Ecuadorian colleges and universities to students who completed undergraduate studies. Term equivalent to Licenciada/o
} 
despite having gained political power and the recognition of diversity in the constitution the last two decades, Indigenous people continue to be the poorest of the poor (Instituto Nacional de Estadística y Censos [INEC], 2016). Education as an indicator of social mobility is not equally distributed in Ecuador. As stated by Gachet et al. (2017), "People who do not speak an indigenous language, live in urban areas and have more money; have more years of education in comparison to those speaking an Indigenous language, live in rural areas and do not have money" (p. 12). Indigenous people receive the lowest formal education in quality and number of years that result in excluding this population from the benefits of public goods (Sánchez, 2006). Even if Indigenous people have access to education, they often obtain the lowest economic returns because of a discriminatory labor market. The Instituto Nacional de Estadística y Censos [INEC] (2016) reported that in 2014, the poverty index for Indigenous people was $64.8 \%$, compared to the Mestizo population, which was reported as $21.2 \%$. The unequal distribution produces social disorder in Ecuadorian society (Sánchez, 2006).

Novo and De la Torre (2010) suggested that in Ecuador, educational institutions are racially segregated, produce differentiated racial and ethnic groups, and allow for little social mobility. They emphasized that the Ecuadorian educational system is plagued by segregation, lack of understanding of cultural diversity, and biased attitudes and practices, especially against underrepresented groups. In schools, Novo and de la Torre recognize discriminatory acts against vulnerable students, noting that they are exhibited to different degrees, ranging from blatant physical abuse to more subtle demonstrations of rejection. Although research (e.g., Brown, 2004; Liao et al., 2016) suggests that manifestations of racism in modern society are not as explicit as they used to be decades 
ago when racism was more intentional-existence undisputed. Modern racism is more implicit, and its expressions have become covert and elaborate (Brown, 2004). The intricate nature of current racist demonstrations results in difficulties in recognizing, assessing, and conceptualizing them (Liao et al., 2016). These subtle aggressive comments or actions against particular groups are microaggressions.

Chester Pierce (1970) first coined the term microaggressions to define slight but harmful snubs mainly experienced by African Americans. Since then, microaggressions have received increased attention in an attempt to identify and eradicate manifestations of inequities. The field of psychiatry first defined the construct of microaggressions; however, the term has been extrapolated to a variety of settings such as the workplace and school. Sue et al. (2007) defined microaggressions as "brief and commonplace verbal, behavioral, or environmental indignities, whether intentional or unintentional that communicate hostile, derogatory, or negative racial slights and insults toward people of color” (p. 271). Messages ascribing to how people look, questioning intelligence, assuming low class, denying racism, and pathologizing cultural values are some examples of everyday microaggressions. Sue et al. (2007) emphasized that microaggressions are subtle and, to some extent, unintentional behaviors perpetrators exert over groups considered as minorities. These apparent inadvertent conducts and their elusiveness make microaggressions challenging to detect and therefore more pervasive in society. Moreover, Sue et al. (2007) conceded that microaggressions constitute a broader spectrum, targeting people by not only their race but also their gender, sexual orientation, or disability. Regardless of the reason why people are targeted or the place where 
microaggressions occur, they contribute to augmenting inequities and the gap between the privileged and the marginalized.

Microaggressions not only affect a range of people but also are present in a variety of scenarios. In the workplace, for instance, microaggressions have considerable repercussions with respect to employment acquisition, job satisfaction, employees' performance, and establishing positive relationships at work (De-Cuir-Gunby \& Gunby, 2016; Young et al., 2018). Microaggressive attitudes seemingly play roles in decreased opportunities for minorities in obtaining jobs in accordance with their experience and preparation. Stereotypes attributed to microaggressions have been shown to result in banning people access to leading positions because most recruiters or managers may be unaware of their biases contributing to perpetuating social disparities (Young et al., 2018). Those having a job also suffer consequences of microaggressions. A microaggressive work environment can result in developing conflicting emotions among people, thereby stimulating a non-inclusive and detrimental atmosphere (Holder et al., 2015). On a personal level, victims of microaggressions at the work place suffer from high levels of stress that interfere in their activities, thereby lowering expected outcomes which may eventually cause employees to quit their jobs (Young et al., 2018).

Educational settings of all levels are also plagued with microaggressions that have a tremendous effect on students. Even though higher education institutions claim to be welcoming spaces for all students, it is at college level where differences can be evident because colleges have traditionally been exclusive for privileged groups not only concerning not only class but race and gender (Smith \& Mao, 2016). Campus 
environments respond to conventional norms where the presence of underrepresented students constitute a challenge to established hierarchies of power. Smith and Mao (2016) also argue that campus policies usually neglect the treatment of issues associated with social order, racism, and classism. Higher education institutions need to provide conditions that make pleasant learning a possibility for all students especially for those coming from disadvantaged or marginalized groups. Smith and Mao (2016) suggest the importance of creating awareness of microaggressions on campus to reduce their occurrence.

\section{Microaggressions and Self-esteem}

Rosenberg (1965) defined self-esteem as an individual's overall positive evaluation of the self. People are constantly assessing their worth and importance on different aspects. If the value people put on themselves is positive, they develop a strong self-esteem that translates in growth and trust in who they are and their sense of worth. Conversely, if the appreciation of value is negative, adverse consequences are expected such as depression, anxiety, oversensitivity, stress, or loneliness (Branden, 1969). Although self-esteem is usually regarded as an internal estimation, it also depends on external factors that respond to the need of humans to fit in a group (Baumeister \& Leary, 1995). An environment where individuals feel they do not contribute, do not accomplish, and do not feel accepted by others, will act as a detrimental factor to self-esteem (Branden, 1969).

Research has documented effects of microaggressions on students' mental health (Nadal et al., 2014; Reid, 2017; Moore, 2012). There is evidence of high levels of distress, depression, low self-esteem and low self-efficacy experienced by students in 
school (Helm, 2013; Nadal et al., 2014) as a consequence of suffering constant hostilities. Nadal et al. (2014) stated how low self-esteem as a result of microaggressions manifests as students experiencing social conformity, reluctance to take part in social and academic activities, fear of giving opinions in public, and insecurity their academic success. Allen (2010) studied the experiences of racial microaggressions among Black middle-class male students. The study suggested that students exposed to racial microaggressions develop low self-esteem because they feel undervalued by their schoolteachers and administrators that give rise to academic failure, high dropout rates, and expulsions (Allen, 2007). Results from the above studies indicate the need to understand the impact of racial microaggressions on students from different populations so schools can assist them in developing higher levels of self-esteem.

\section{Microaggressions and Self-efficacy}

People like to exhibit confidence and control over their behavior when trying to accomplish a goal. Bandura (1977) referred to self-efficacy as the conviction on one's capacity to conduct actions required to produce specific performance attainments. Selfefficacy is reflected in people's belief to thrive in a given situation. He also described this confidence on aptitudes as a powerful determinant factor of how we think, behave and feel that consequently affects countless states such as psychological, behavioral, and motivational. Self-efficacy is a crucial element when individuals engage in choosing goals, the actions they take to achieve those goals and the way they take account of their performance.

Individuals develop self-efficacy in their interactions with different environments. At school, students can display performances that can determine the enhancement of their 
self-efficacy. Educational situations are the primary sources for students to acquire many competencies (Bandura, 1994). While instruction develops content knowledge and problem-solving skills that enable students to perform successfully in a variety of circumstances, the sole act of instructing students does not result in developing their selfefficacy. On the contrary, self-efficacy depends heavily on social factors (Bandura, 1994). Observing successful peers performing determined skills at school raises student's beliefs that they are also capable of success. Another source to stimulate students' selfefficacy is verbal persuasion. By persuading students with respect to their abilities, teachers can strengthen their confidence to accomplish a goal. Students who believe in their capabilities are likely to persist in their purposes (Bandura, 1977).

An ideal school environment supports student's self-efficacy. Unfortunately, the school is often the place where students receive negative messages attributed to their race or social status that affect their self-efficacy (Pedditzi \& Passarelli, 2018). Students' selfefficacy decreases when they are immersed in racialized environments. Receiving harmful comments or experiencing negative attitudes from teachers and peers, impacts students' capacity to trust their capabilities to perform at school. McCullagh (2007) looked at the association between racial microaggressions, college self-efficacy and persistence of students of color. Findings from the study suggested that self-efficacy is closely related to the social and academic integration of students. Similarly, students with high levels of self-efficacy achieved noticeable academic and intellectual performance that helped them navigate college effortlessly. A healthy environment where students' self-efficacy beliefs are encouraged positively also determines the persistence students demonstrate to accomplish their academic pursuit (McCullagh, 2007). 
As self-efficacy constitutes a robust predictor of academic success and persistence, educational settings should recognize the importance of developing selfefficacy in students and work to promote its enhancement especially in students who by virtue of their race or social status are subject to microaggressions.

\section{Reacting and Coping with Microaggressions.}

There is no doubt that experiencing racism takes a high emotional toll on its victims (Lowe et al., 2012). As soon as a microaggressive incident takes place, the target generates a reaction that, according to Sue et al. (2007), implies formulating a series of questions that victims ask themselves in their intent to understand and assimilate the event. Questions like “Did what I think happened, really happen?” Was this a deliberate act or an unintentional slight? How should I respond? Sit and stew on it or confront the person? If I bring to topic up, how do I prove it? Is it really worth the effort? Should I just drop the matter?" (p. 279) emerge in the mind of the target. Sue et al. (2007) link these questions to people determining if the microaggressions really happened by establishing patterns with previous events in different contexts. The questions lead the individual to choose between two options, doing nothing or responding with anger. Deciding to do nothing about the incident is an alternative that certainly produces the most psychological harm to the victim. A passive attitude develops feelings of anger and frustration that mine the person's mental balance. On the contrary, an active decision of responding to the episode with anger, may result in increasing levels of hostility and tension between people that ultimately affects the victim who is seen as hyper-sensitive or apprehensive (Sue et al., 2007). 
Regardless of the reaction to a microaggressive event, people seek opportunities that help them deal with incidents of discrimination. Establishing mechanisms to contend with the costs of microaggressions is crucial because doing so helps people shield themselves against feelings of humiliation, marginalization, and frustration (Holder et al., 2015). Research has documented strategies people adopt to alleviate racial pressure such as getting involved in religious activities, developing spirituality, armoring, shifting, engaging in supporting networks, funding sponsorships, mentoring, and self-care (Holder et al., 2015). In the case of college students, they engage in creating methods to deal with the marginalization they are subject to on campus. Lewis et al. (2013) determined how Black women faced microaggressions by generating approaches that implied resistance, collective coping strategies and self-protective coping strategies. Examples of some of the devices used by Black women included using their voice as power, resisting Eurocentric standards, leaning on one's support network, becoming a Black superwoman $^{2}$, becoming desensitized, and escaping. Results from Lewis' study indicated that Black women used these coping strategies according to different contextual factors that determined the type of strategy they used.

Gonzalez (2002) looked at the experiences of Chicano students in a predominantly White university in terms of the challenges they faced as college students. The study reported that one of the most salient difficulties students faced was the constant message that Chicano students received in regard to their culture as not valued and unwelcomed. To contest the racialized campus environment, students opted for adopting

\footnotetext{
${ }^{2}$ Image of a strong woman who is resilient, successful and committed.
} 
approaches of cultural subsistence that helped them navigate the university. The actions students took were useful for reconstructing their culture and sense of selves (Gonzalez, 2002). Other studies such as Wright (2016) exploring strategies students take to battle hostile campus environment, include forming groups where people share similar interests, values, food, music, and cultural symbols aimed to improve their sense of belonging and acceptance. These studies suggest that the actions taken by students to fulfill their psychological needs, equate in some sense with Maslow's (1954) hierarchy for human motivation. The actions described in both studies may be associated with psychological needs such as relationships, friends, prestige, and feeling of accomplishment as indicated in Figure 1 Maslow's hierarchy of needs.

\section{Figure 1}

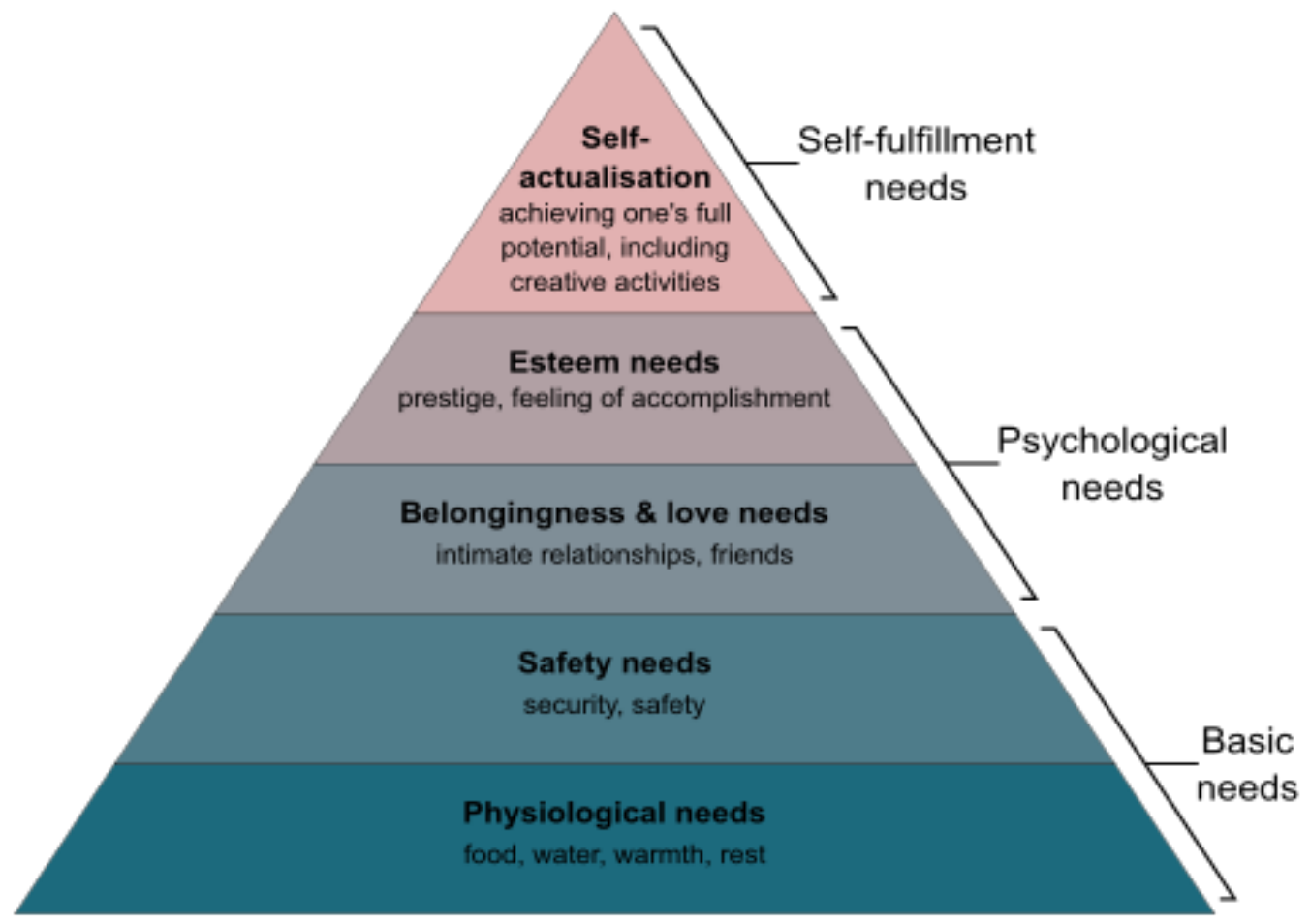

Maslow’s Pyramid of Psychological Needs 


\section{Indigenous Populations and Microaggressions in Ecuador}

The concept of microaggressions is relatively novel. Since the release of the seminal article by Sue et al. (2007), microaggressions have sparked attention in fields such as education, sociology, and psychology (Wong et al., 2013). However, most of the literature around microaggressions has originated from developed countries. Studies have examined the impact of microaggressions on certain populations in particular African American, Latino, Asian American and a variety of underrepresented groups in reference to gender, sexual orientation, class, or phenotype (Wong et al., 2013). Despite microaggressions being present around the world, there is scarcity of studies in regions where this phenomenon is unknown and therefore overlooked.

Hollenstein (2008) contended that in Ecuador, race influences the internal dynamics at schools. The author described schools as racialized environments where symbolic tensions such as racial hostilities take place. However, the phenomenon of microaggressions has not received the attention it deserves despite its prevalence. Private and public institutions have attempted to address the issue, but their intentions lack the support of empirical research. In 2017, the Casa de la Cultura Ecuatoriana (Ecuadorian

Culture Board) launched a campaign called Microaggressions-Macroimpacts aimed to make high school students aware of the impact of verbal misconduct against women. Likewise, the Universidad San Francisco de Quito introduced a uni-diversity program to sensitize and facilitate students' ability to recognize and avoid microaggressions. The program emphasized the need for victims to identify the perpetrators who most of the time are people with more privilege to derogate, devalue, or diminish other people's abilities. The campaign stated that in Ecuadorian cultural context, lighter skin is more 
appreciated than darker skin and that this racist expression produces harmful effects on people (Enfoque Universidad San Francisco de Quito [USFQ], 2017). The report asserted that Indigenous people, Afro-Ecuadorians, and darker Mestizos are less valued and therefore frequently being the targets of discrimination in the form of microaggressions.

Roitman and Oviedo (2016) contended that institutions where Mestizo students are in the majority neglect ethnic minority groups such as Indigenous students whose presence is overlooked. In the same vein, Martinez and de la Torre (2010) claimed that the Ecuadorian educational system reflects a society where segregation and hierarchical structures are one of the most restrictive in Latin America. Issues associated with racism such as discrimination, disenfranchisement, injustice, and microaggressions are common in schools and university campuses where Indigenous students navigate their academic endeavors in a Mestizo majority environment. From these mistreatments, microaggressions have not been subjected to analysis partly because one people believe they do not constitute harm and two because victims are not aware of racist manifestations affecting them (Friedlaender, 2018).

In 2004, the National Office of Social Affairs in Ecuador launched a racial and discrimination survey to determine the sociodemographic and socioeconomic state of Afro-Ecuadorian and underrepresented populations and to assess the prevalence of racial issues in the country. The report Racism and Racial Discrimination in Ecuador (2005) indicated that $78 \%$ of all Indigenous people in the country do not have enough understanding regarding racism, its manifestations and its impact on their lives. ${ }^{3}$

\footnotetext{
${ }^{3}$ Theme 1: Perception of Racism. Survey applied to 37,519 participants. SISPAE
} 
Interestingly, the report Racism and Racial Discrimination in Ecuador (2005) stated that only $52 \%$ of the Ecuadorian population has a clear notion of racism. People who have achieved a college education, with high-income and living in urban areas are the most informed. The report concluded that people in Ecuador, especially those suffering the consequences of racism, are not aware of the impact it has on their lives. Likewise, the report argued that people invalidate racism by mainly associating it with colonial history.

Discussing issues related to racism generates resistance because race is still a taboo topic (Beverly, 1992). The reasons for resistance are partly the result of assuming that social harmony should prevail. Hence, people trying to address racial issues are considered to be spreading social conflict. The discussion of racial matters creates discomfort and powerful emotional responses people are not usually willing to accept (Beverly, 1992). Thus, for the educational system, it is safer to overlook Indigenous students' lives and struggles rather than taking the risk of unlocking the multiple intersections and hassles, they face because of their ethnicity. Roitman and Oviedo (2016) suggested that unless these issues are acknowledged by Ecuadorian society, aggressive and unfair treatment against minorities will prevail.

\section{Purpose of the Study}

The current mixed methods study aims to examine the types of microaggressions affecting Indigenous undergraduate students while pursuing a bachelor's degree in a public higher education institution in Ecuador and the associations these microaggressions have on their self-esteem and self-efficacy. It also aims to explain the experience of Indigenous students with respect to how they respond to, react to, and deal 
with microaggressions. These purposes gear towards giving voice to students and creating awareness of the impact that racialized hostilities have on students' lives. The study also embraces ideals of social justice in Ecuadorian society by advocating for Indigenous students.

The purpose of the study is supported by Nadal (2011) who recommended inquiry into finding relationships between microaggressions and different psychological constructs that might hinder underrepresented students' wellbeing such as symptoms of depression, anxiety, or low self-esteem. Similarly, Sue et al. (2007) emphasized the need to make concealed hostile events affecting minorities visible so it is easier to battle them. To achieve this goal, they also suggested openly and honestly engaging in a dialogue about race and racism, especially in educational settings. Furthermore, Nadal (2011) highlighted the importance of developing studies in contexts and populations other than those identified in the United States. Through evidence of how microaggressions manifest in a variety of settings, determining additional elements related to racialized hostilities, as well as different implications to those already identified, may be possible.

Sue et al. (2007) also remarked that providing marginalized groups opportunities to reflect on their own identity, recognizing biases and prejudices from people around them that have an impact on their lives, are important skills for them to develop. In an Ecuadorian context then, we may extend their argument to suggest that Indigenous students need to develop racial self-awareness that equips them to identify microaggressions in their environment; thereby they might interrogate themselves about prejudice to develop ways which ultimately reduce its impact in their own lives. Indigenous students unaware of these phenomena may also need to know how these 
subtle manifestations have a detrimental impact on their psychological well-being. Finally, Nadal (2011) suggested that it is critical that students assess their responses and the type of agency they take when being targeted by microaggressions because they both have outcomes in their lives.

Given the scarcity of research exploring microaggressions in Ecuadorian educational contexts and the complexity of the topic, mixed methodologies to analysis were appropriate to my study. Creswell (2008) suggested that by using mixed methods, researchers can have a more informed understanding of the problem than using either quantitative or qualitative approaches separately. Additionally, mixed methodologies focus on social justice, advocacy, and concern for the improvement of the human condition (Sweetman et al., 2010). In my study, the quantitative approach (survey instruments) determined the most prevalent types of microaggressions Indigenous students encounter and their association with self-efficacy and self-esteem. In the meantime, qualitative methods, namely interviews, were suitable because they aimed to "explore and understand the meaning individuals or groups ascribe to a social or human problem" (Creswell, 2009, p. 3). Conversations also determined the ways students respond or react to microaggressions, as well as the mechanisms they use to cope with the identified hostilities.

My study contributed to the understanding of Indigenous students' experience and perceptions with microaggressions in that uncovering these issues in Ecuadorian higher education was necessary towards achieving more stress-free, healthy, and equitable environments. 


\section{Research Questions}

The goal of my study is in examining racial microaggressions as they associate to Indigenous undergraduate students' self-esteem, self-efficacy, responses and mechanisms they use to cope with hostilities while they pursue a Bachelor's degree in a public higher education institution in Ecuador.

The study aims to answer the following research questions:

1. What are the most/least common types of racial microaggressions that Indigenous students experience while pursuing a degree in a public higher education institution?

2. What is the association between microaggressions Indigenous students' selfesteem?

3. What is the association between microaggressions Indigenous students' selfefficacy?

4. How do Indigenous students experience racial microaggressions while pursuing a degree in a public higher education institution on issues of responses to microaggressions and mechanisms they use to cope with microaggressions?

\section{Significance of the Study}

Investigating issues related to oppression is a continuing concern within the field of education. Microaggressions as a form of discriminatory behavior are detrimental for students since, according to the literature, they have an impact on their mental and physical well-being. Microaggressions also contribute to creating an unwelcoming school environment that promotes tension among students. Given the scarcity of research on 
microaggressions, the results of this study added to the body of knowledge on to how the phenomenon of microaggressions manifests in higher education institutions in Ecuador.

Nadal et al. (2014) reveals the need to conduct research that leads to the identification of the types of microaggressions as a condition to prevent their occurrence and decrease harmful effects on targets. Individuals, who recognize harmful snubs in their environment, are able to reduce the impact these hostilities represent to their mental and physical well-being by adopting appropriate responses and coping behaviors that in turn help them reduce the toll hostilities take on their lives. In the case of microaggressions in educational settings, the literature reveals the need for studies that contribute to raise awareness of issues related to race, diversity, challenges underrepresented students face as a result of being exposed to racial slurs, and the importance of providing all students with safer educational environments where everybody feels welcomed. Additionally, Wong et al. (2014) stressed that, as a novel field of research, microaggressions are a feasible area that require conceptual development and the use of varied methodologies of study.

The findings may become a benchmark for institutions to design strategies to educate the community on the importance of talking honestly about racism instead of adopting a color-blind stance.

The study intends to open spaces to know more about the Indigenous students, their hassles, experiences, and voices so institutions can effectively serve this population. More than ever before institutions where indigenous students are an ethnic minority need to be more aware and informed of their racialized issues, so they enact agency in supporting at-risk students in their academic endeavors. 
The findings of my study will redound to the benefit of Ecuadorian educational system considering that racial microaggressions are deeply rooted in all levels of schooling. The study will open opportunities to further research on issues such as devising instruments to measure racial microaggressions for Indigenous populations, understanding the impact of racial hostilities on Indigenous students' academic achievement and engagement, and exploring acculturation processes that students undergo in their effort to fit in their institutions. The study considers that presence of oppressive manifestations against Indigenous students justifies the need for reflecting about the multiple challenges they encounter in their search for success.

\section{Theoretical Framework}

By looking at microaggressions Indigenous students experience while pursuing a college degree, the study advocated for social justice in Ecuadorian society in general. Because inequalities are present in the Ecuadorian educational system, there is need for research to include an advocacy perspective that calls for change. My study adopted a transformative framework in that the construction of knowledge should not be neutral but predisposed to aid people improve society (Sweetman et al., 2010). Since microaggressions are manifestations of oppression and domination, in order to achieve the goals of my study, I used the five faces of oppression as the lens to analyze microaggressions which is a form of discriminatory behavior toward members of an oppressed group. Young (2004) offers a comprehensive framework to identify oppression and how it is exerted on people who are systematically marginalized. Young's framework was used in my study to examine the likelihood of Indigenous students to be oppressed as a group by virtue of their ethnicity while pursuing their bachelor's degree. 
In her analysis of oppression, Young proposes five categories that indicate the existence of oppression of a group namely exploitation, marginalization, powerlessness, cultural imperialism, and violence. The rationale for choosing the five faces of oppression to analyze microaggressions in Indigenous students in Ecuador relied in the fact that the criteria facilitate the objective identification of oppression students are subjected because they belong to a specific group. Young's framework gave my study the possibility to operationalize each criterion by using different artifacts such as observable behavior, status relationships, distributions, texts and other cultural items. Another advantage of using Young's framework in the analysis of Indigenous students' microaggressions was that the presence of any of the five conditions, is sufficient for the group being oppressed (Young, 2004).

- Exploitation: The history of humanity is plagued of references of groups exploited by others for a variety of reasons. Young (2004) describes exploitation as "[occurring] through a steady process of the transfer of the results of labor of one social group to benefit another" (p. 46). According to Young (2004), exploitation derives in unequal relations where "the energies of the have-nots are continuously expended to maintain and augment the power, status, and wealth of the haves" (p. 46). Young asserts to say that race is a basic source of exploitation because "wherever there is racism, there is the assumption, more or less enforced that members of the oppressed racial groups are or ought to be servants of those, or some of those, in the privileged group" (p. 48). Young goes further to clarify that exploitation does not only refer to labor exclusively but also the loss of control and self-respect that oppressed groups suffer because of their condition. 
- Marginalization: The concept refers to societies creating groups considered as underclass. Underclass people are condemned to living in social marginality because the system does not use their labor force. Marginalization emanates from different sources such as age and physical and mental health, but mostly race. Young deems marginalization as the most dangerous type of oppression a group can face because it bans people from useful participation in social life resulting in material deprivation that results in extermination. Societies acknowledge people's deprivation caused by marginalization and have tackled it by establishing welfare programs. Although the nature of these programs is to help the marginalized to mitigate their starvation, they create dependency on people since they have to accept authoritarian conditions, punitive acts or arbitrary treatment for the sake of maintaining their government subsidy. As dependents of the system, marginalized people lose their freedom to decide about what is good for their lives and accept being controlled by others. Consequently, people are deprived of their freedom.

- Powerlessness: Society also acts as a buffer to control people's power. Another indicator of oppression is impeding or precluding people from participating in decision-making. Young describes powerless people as those who "do not regularly participate in making decisions that affect the conditions of their lives and actions" (p. 52). In the same vein, powerless people "are situated so that they must take orders and rarely have the right to give them" (p. 52). For oppressed people, lacking power condemns them to a life of discourteous treatment because of the status they occupy in society. As a consequence, lack of power generates a series of associated injustices that have an impact on the life of people. 
- Cultural Imperialism: Young categorizes exploitation, marginalization and powerlessness under the umbrella of relations of power and oppression resulting from the social division of labor that delineate people's access to material resources. However, Young identifies another type of oppression that is not attributed to material resources: Cultural imperialism deals with one group imposing the perspectives, ways of living, ways of knowing, and other manifestations of culture to another group considered insignificant or worthless. The worthless group is invisibilized and stereotyped since it is outside the norm. Invisibleness acts as a way to deny people's essence or identity while stereotyping confines people to attributes they cannot easily deny. Young provides some examples of stereotypes like "gay people are promiscuous, Indians are alcoholics and women are good with children" (p. 55). Moreover, the dominant group enforces acceptance by establishing standards that have to be accepted as the norm. Those who contradict or are outside the norm are considered inferior, and their experiences are deemed unimportant; thus, they are oppressed for being who they are. The oppressed groups follow the archetype group in their attempt to fit in the norm. Young explains cultural imperialism as that "the oppressed group's own experience and interpretation of social life finds little expression that touches the dominant culture, while the same culture imposes on the oppressed group its experience and interpretation of social life" (p. 56).

- Violence: Young sees violence as a systemic social practice where oppressed people are subjected to violence for the simple fact of being members of a group that has been assigned as underclass. Violence comprises "not only direct 
victimization, but in the daily knowledge shared by all members of oppressed groups that they are liable to violation, solely on account of their group identity" (p. 57). Oppressed people live in a constant threat that substantially affects their freedom and dignity making them spend a lot of physical and mental energy to cope with it. 


\section{CHAPTER TWO}

\section{LITERATURE REVIEW}

This chapter consists of a review of the literature in reference to microaggressions, definitions and types, associations between microaggressions and mental well-being with regard to self-esteem and self-efficacy as essential pillars of the image that people have of themselves. Additionally, this chapter explores different responses individuals give when subjected to microaggressions, and mechanisms they adopt to deal with racialized subtle assaults. Next, this review examines literature related to Indigenous populations, schooling and microaggressions and salient aspects of Ecuadorian schooling system. The final section of this chapter looks at the model proposed by Young (2004) in reference to the Five Faces of Oppression namely exploitation, marginalization, powerlessness, cultural imperialism and violence. The model of the Five Faces of Oppression serves as the lens to analyze the practice of power society exerts over certain groups to maintain privilege of other groups.

\section{Microaggressions}

\section{Definition and Types of Microaggressions}

Since its onset in 1970, the concept of microaggressions has become a useful tool for researchers to examine issues related to racism that influence people who belong to racial minorities (Perez \& Solórzano, 2015). Pierce (1970) first used the term microaggressions in the field of psychiatry to refer to subtle offensive mechanisms towards African American targets. The author later expanded the definition as "subtle, stunning, often automatic and non-verbal exchanges which are put-downs of blacks by offenders" (Pierce et al., 1978, p. 66). The purpose of such microaggressions is to 
gradually limit and reduce the spaces of disadvantaged people so they do not invade those of the privileged - in other words, to put people of color into their place with incessant messages that they are insignificant and irrelevant (Pierce, 1969). The implicit racial derogatory lessons that constrain other people's spaces are, according to Pierce (1988), present in all kind of settings such as workplaces, campuses, and schools, invading a variety of people's interactions with consequences that go from augmenting feelings of degradation to eroding self-confidence and self-image. In his original work, Pierce (1988) intended to fill the gaps left by psychology in addressing the specific needs of African American people suffering from an array of mental issues because of their exposure to constant racial incidents.

The most extended definition in the literature for microaggressions that will be used along this study comes from Sue et al. (2007). They asserted that racial microaggressions are "brief and commonplace daily verbal, nonverbal, behavioral, or environmental slights, snubs, or insults, whether intentional or unintentional, that communicate hostile, derogatory, or negative messages to target persons based solely upon their marginalized group membership" (p. 271).

Microaggressions go beyond offenses, insensitive remarks, or generalized negative stereotypes about people. Microaggressions deal with people's disparagement against others by virtue of their membership in a group that is discriminated. However, the core issue of microaggressions is that they occur casually, recurrently, and without any intended harm by perpetrators, but such unintended insults eventually have negative impact on the victims' lives. Victims describe microaggressions as undergoing damage that compromises their existence in multiple ways. 
Although the original foundational concept of microaggressions has not changed over the years, it has become the catalyst for numerous studies. These studies have added more nuances to understanding this concept. Kohli and Solórzano (2012) added new elements to Pierce's definition as

Subtle verbal and non-verbal insults/assaults directed toward People of Color often carried out automatically or unconsciously; layered/insults/assaults, based on one's race, gender, class, sexuality, language, immigration status, phenotype, accent, or name. Cumulative insults/assault that take their toll on People of Color. In isolation, racial microaggressions may not have much meaning or impact; however, as repeated slights, the effect can be profound (p. 447).

Kohli and Solórzano's (2012) definition categorized microaggressions as subtle.

This explains why people, especially victims, have difficulties analyzing or describing them. Microaggressions are so elusive that they often appear to be a compliment or a joke but contain a hidden detrimental message about people. Elusiveness may explain why this phenomenon is deeply rooted in society but unnoticed at the same time. In view of the subtle nature of microaggressions, both perpetrators and victims are unaware of their occurrence. As a result, microaggressions are seen as "normal" behaviors that do not fall into the category of harmful hostilities. This vagueness is what Sears (1988) identified as symbolic racism, responsible for ascribing people as second-class citizens. Another salient feature in this definition is that victims of microaggressions are picked for being different or for challenging what society or majorities consider as acceptable.

Furthermore, given microaggressions are cumulatively harmful, targets are more likely to experience negative permanent emotional, cognitive, behavioral, physiological, and social effects that can manifest in a variety of forms (Sue, 2010). 
Sue et al. (2007) first identified three forms of microaggressions labeled as microassault, microinsults, and microinvalidations. Microassaults are explicit and conscious manifestations of racism that take place when offenders believe they are in a safe situation. Microassaults are common in private situations (micro) where people share the same racialized biases where a group supports the offender's opinions. Microassaults reveal characteristics of conventional practices of discrimination with the only difference being that they are not open proclamations of racialized preconceptions. For instance, saying "that is typical of Indigenous people," suggests that there is something wrong about being Indigenous and that, although people are perfectly aware of the meaning of the words, they may not acknowledge being racist. However, the comment can be offensive for people belonging to this ethnic group. Racial jokes, intended name-calling, avoidant behavior, and purposeful discriminatory actions fall in the category of microassaults (Sue et al., 2007). Jokes ascribing race are common and socially accepted in Ecuadorian environment. These jokes usually ascribe to people's phenotype, customs, clothes, and language. For instance, in a conversation between Mestizo friends, someone would say "Comí como Indio.” Mestizo listeners would laugh in the implicit messages that Indigenous people have bad eating manners, eat a lot, or eat food that is characteristic of Indigenous people's diet. Indigenous people may also be subject to avoidant behavior especially in public transportation where they are usually asked to sit at the back.

Microinsults are unintentional, rude, and insensitive messages against target people. Although these messages are involuntary for the offender, they communicate a concealed derogatory message to recipients who see their racial culture or identity as less 
valued (Sue et al., 2007). Stating that someone occupies a position because the quota for underrepresented groups and Indigenous nationalities had to be filled is a clear example of a microinsult. Such an assertion implies that the person does not have sufficient merits to fulfill a position other than being Indigenous or Afro-Ecuadorian. The case above falls in the category of verbal microinsult. As mentioned previously because microaggressions are unintentional and most of the time difficult to recognize, they are quite common within work and school settings. Nonverbal microinsults also take the form of avoiding eye contact with target people or considering their opinions irrelevant (Sue et al., 2007). Examples of microinsults in educational settings are numerous. Typically, Indigenous students are seen as slow learners. They are also expected to be silent and submissive. If an Indigenous student excels academically, such is seen as abnormal. If the student speaks loudly or expresses their opinion to contradict a given viewpoint, this behavior is considered as going against Indigenous nature.

Microinvalidations are also a rampant form of microaggressions, usually associated with colorblindness. Colorblindness refers to society beliefs that racism is no longer existent as a problem and that everybody has equal opportunities to succeed despite their skin color. A colorblind attitude prevents people from seeing the causes of racial inequality and how it affects people. Colorblindness acts as a buffer that "resolves the tension of endorsing racial equality in a social structure still designed to preserve White advantage" (Mueller, 2017, p. 221). Microinvalidations occur when people adopt colorblind approaches that "exclude, negate, or nullify the psychological thoughts, feelings, or experiential reality of a person of color" (Sue et al., 2007, p. 274). Ignoring the effects of discrimination that people suffer because of their race, social status, or 
gender and the interest of some groups to maintain their privilege, results in the prevalence of dominance of hegemonic ideologies. Sue et al. (2007) also highlight that racism as a nonexistent phenomenon is a widespread opinion that minimizes the existence of biases against African American people in the United States. Similarly, there are widespread criteria that underrepresented people should not complain anymore about exclusion because they receive affirmative action and extra benefits for belonging to a group identified as at risk (Sue et al., 2007). These policies to relieve historical oppression are supposed to minimize the challenges dominated people face because of discriminatory behaviors against them. Examples of microinvalidations are when people receive comments such as "We do not see color anymore," "You overreact when people talk about race," or "There is no racism because the constitution assigns the same rights to all citizens."

In Ecuador, people deny the existence of racism. If they acknowledge any traces of racism, they associate them to events of the past. Roitman and Oviedo (2017) cite examples of people microinvalidating racism in Ecuador such as "I don't think there is still racism in Ecuador ... Although we must consider that it is a natural human tendency to try to have one's own group. But racism, racism in Ecuador, I have not seen!" (p. 2275). In this quote, the speaker pretends to minimize the existence of practices that discriminate individuals because of their race and ethnicity.

The three forms of microaggressions coined by Sue and colleagues (2007) led to the identification of nine categories as follows: alien in one's land, ascription of intelligence, color blindness, criminality assumption, denial of individual racism, myth of meritocracy pathologizing cultural values/communication styles, second-class status, and 
environmental invalidation. The authors state that these categories relate to one another and are indicators of the prevalence of racial biases in a society that maintains underrepresented groups at a disadvantage compared to majorities interested in preserving their hegemony. The three forms of microaggressions with the corresponding categories identified by Sue and colleagues have been subject of numerous studies that have added more elements to the recent literature on microaggressions (Allen, 2010; Burdsey, 2011; Constantine, 2007; Gomez et al., 2011; Wang et al., 2011).

The taxonomy developed by Sue and colleagues (2007) was also used as the foundation to create the Racial and Ethnic Microaggressions Scale (REMS) proposed by Nadal (2011). This quantitative instrument measures racial microaggressions that people experience in their everyday lives in six components: assumptions of inferiority, secondclass citizenry and assumptions of criminality, microinvalidations, exoticization, assumptions of similarity, environmental microaggressions and workplace and school microaggressions. The REMS is an instrument that has been implemented in an effort to assess racial microaggressions on the basis of three attributes: 1) The items correspond to conceptual ideas related to racism; 2) The scale correlates with other scales researchers used to measure issues of racism and discrimination; and 3) People who have taken the test have been able to identify the intention of the instrument to determine racism and discrimination (Nadal, 2011). (See REMS questionnaire by components Appendix A).

\section{Associations between Microaggressions and Mental Well-being}

Kolhi and Solórzano's (2012) vision of microaggressions reveal the profound toll hostilities take on people. The effect racial microaggressions have on psychological wellbeing is high with adverse consequences. People tend to avoid engaging in overt 
manifestations of racism because they lead to immediate reactions of rejection as they are seen as socially unacceptable behaviors. More than ever before, people are more politically correct and abstain themselves from involving in acts that result in public disapproval. In consequence, explicit conscious aggressions have seen an important decline in the last few decades (Nadal, 2008). Although this decline is a positive step towards living in a safer environment, subconscious expressions of racial biases are still deeply ingrained in society although virtually hidden. These biases result in people perpetrating microaggressions that, no matter how subtle, have profound long-lasting outcomes on recipients' physical and psychological well-being. The effects are translated into feelings such as unhappiness, loss, and failure. Another consequence of microaggressive actions is that they contribute to augment levels of racial anger, frustration, and low self-esteem (Solórzano et al., 2000).

Research has provided robust evidence that suffering from discriminatory acts results in high levels of distress that can take the form of chronic life stress, especially for African American people. There is also an undeniable indication that the persistent exposure to discriminatory occurrences can have as extreme effects on people's mental illness compared to those produced by a single life traumatic event (Kessler et al., 1997).

Wang et al. (2011) studied the association between racial microaggressions and the emotional consequences they generate. Results of this study suggested that as an underrepresented group, Asian Americans experienced high levels of emotional outcomes derived from being treated differently not necessarily negatively because of their race. To test if race was a determinant factor in generating differences in the way Asian Americans were addressed by other people, Wang et al. (2011) introduced in their study 
other variables such as gender, height, weight, age, and social class. The study demonstrated that race is the main factor associated with treating people in a different manner, which in turn links both negative externalizing of emotions (anger, frustration, resentment, and scorn) and internalizing emotions (anxiety, embarrassment, sadness, and shame). Participants also reported other emotions like confusion and disappointment. The study concluded that despite multiple social identities that contribute to the appearance of different emotions, a race-relevance appraisal is a central factor in generating negative emotions. The study called for more studies that examine experiences of different groups with microaggressions to determine associations between slurs and their effect on people's lives.

People do not usually acknowledge having racial biases. People believe that being neutral and avoiding their involvement in open discriminatory events makes them racially harmless. However, people may not be aware of hidden biases that result in adopting negative racialized behaviors. Unintentional and repeated actions that convey undermining messages certainly have a profound impact on people's lives for they accumulate negative emotions that compromise their mental health in the long term. Nadal et al. (2014) looked at the impact of racial microaggressions on the mental health of people of color attending to counseling services. The study measured the six subscales proposed in the REMS referred to as assumptions of inferiority, second class citizen/criminality, microinvalidations, exoticization /assumptions of similarity, environmental microaggressions, and workplace and school microaggressions. The six categories were contrasted with the shorter version of the Mental Health Inventory (MHI) to establish possible associations between all variables. The study concluded that 
exposure to frequent racial microaggressions predicts people's mental health issues.

Results indicated that people experiencing racial microaggressions in their daily lives were more prone to develop negative mental health symptoms such as depression, anxiety, negative view of the world and lack of behavioral control (Nadal et al., 2014). Nadal and colleagues (2014) acknowledged the need for further research on the effects of microaggressions on people living in different geographic locations, educational background, age, and gender. Similarly, the study recommended developing more research to analyze other variables that might be associated with microaggressions such as self-esteem, personality, and social support.

Microaggressions have been found to be detrimental for mental health and wellbeing of members of marginalized groups. In the long term, the accumulation of microaggressions can also affect physical health. Several studies have reported racism as a contributor to disparities in health, especially for African American and marginalized groups (Clark et al., 1999; Fisher \& Shaw, 1999; Rollock \& Gordon, 2000). Williams, Neighbors, and Jackson (2008) looked at empirical evidence on the association between racial/ethnic discrimination and health. The study indicated that discrimination is strongly associated with numerous markers of poor physical and mental health. Mental health suffers in aspects such as well-being, self-esteem, control, psychological distress, depression, anxiety, and increased levels of anger. In reference to physical health, studies analyzed reported people suffering from blood pressure and cardiovascular conditions as the result of exposure to discrimination. Overall health behavior consequences were also reported in the form of the increase of smoking and alcohol consumption (Williams et al., 2008). The study highlighted gaps in the literature including limitations in the 
measurement of discrimination, research designs, and the association between prejudice and health over life stages. Williams et al. (2008) recommended studies that focus on individual experiences to assess the type of stressors that affect health as well as coping resources people use to deal with discrimination. In this regard, qualitative approaches and the use of personal interviews were considered as alternatives for researchers to obtain more detailed evidence of how discrimination manifests on people's physical and mental health.

Nadal et al. (2017) explored the correlation between racial microaggressions and physical health problems. As in other studies, the REMS was used in conjunction with the 36-item short form of the health survey (RAND). The results suggested that the exposure to frequent racial microaggressions has detrimental effects on physical health, especially to functions related with the ability of individuals to develop their duties because of the increase of physical and emotional difficulties. Another finding indicated that people exposed to racial hostilities reported to have less energy, poorer emotional well-being, lower social functioning and higher levels of pain that interfere with their activities. The study also concluded that being microaggressed at school and the workplace limits the roles of students as they experience health issues, emotional impairments, and problems with their social relations. Nadal and colleagues (2017) called for the need to educate people so they identify the multiple subtle discriminatory events that surround them and how these events have an effect on their lives. Finally, Nadal et al. (2017) emphasized the importance of people to be conscious of the different expressions of racial hostilities. The authors believe that the understanding of racialized manifestations will help targets adopt mechanisms to better cope with aggressions. 
Overall, the literature confirms several detrimental effects racial discrimination in the form of microaggressions has on people's mental and physical well-being specifically on central aspects like self-esteem and self-efficacy. As a practice to degrade people, racial microaggressions do not occur in isolation. They denote complex intricate underlying implicit biases that plague society contributing to social disorder at all levels. Here is why microaggressions need more attention.

\section{Microaggressions and Self-esteem}

The way other people perceive us, their reinforcements, and evaluations are crucial to how we construct our self-concept. Self-concept is the assembly of different factors shaped by our experiences and interactions with the environment where people's opinions and judgments about us play a decisive role (Schunk, 2012). One of the components of self-concept is self-esteem.

Self-esteem is one of the most complex human traits to define, given the various implications it entails. At its simplest level, self-esteem is defined by Merriam-Webster (n.d) dictionary as "the confidence and satisfaction in oneself ." In Sedikides and Gress (2003), self-esteem is described as “the individual's opinion or a particular view of one's own self-worth, self-respect and self-confidence and the extent to which the individual considers positive or negative opinions about self." These opinions are closely related to people's personal beliefs about their skills, abilities, and social relationships. Similarly, Schunk (2012) described self-esteem as the extent to which individuals accept and respect themselves. In other words, self-esteem is one's perception of personal value and appreciation. Having an adequate perception of ourselves is crucial since it helps us explore our real capabilities and function at our full potential. 
Brown et al. (2001) conceptualized self-esteem as a tridimensional attribute. First, global self-esteem refers to how people subjectively feel about themselves, the feelings of appreciation for themselves. This type of self-esteem is stable despite time and situations. A second form of self-esteem deals with the self-evaluation that relates to the way people appraise their skills and qualities. In this sense, self-esteem derives in concepts such as academic self-esteem and social self-esteem. The third view of self-esteem comprises the feelings of self-worth that may vary according to temporary emotional states that mainly depend on the particular circumstance the individual is experiencing. In this regard, the individual experiences high levels of self-esteem when a positive event occurs and low levels when facing adverse situations (Brown et al.,2001, p. 616).

As an instrument to self-evaluate ourselves, self-esteem is an all-inclusive indicator that allows people to appraise their self-worth and the affective reactions that their own judgments generate (Murphy et al., 2005). Consequently, self-esteem is the mechanism that helps people evaluate themselves and generate an emotional reaction towards themselves that can be positive or negative. The idea of self-esteem as an evaluative mechanism individuals use to judge themselves is present in almost all descriptions and philosophies that have analyzed this construct throughout the years.

Hewit's (2002) contribution to theorizing self-esteem surpassed the psychological perspective from which self-esteem is usually examined. Hewit contended that selfesteem is tied to social conditions. In their interactions with their surroundings, individuals learn to experience and deal with self-esteem. Learning and coping occur in expected social circumstances and is governed by social conventions. The social context then is relevant in shaping people's self-esteem. Therefore, both the internal and external 
components of self-esteem cannot be separated because there is a reciprocal influence between one and the other.

For the purpose of this study, self-esteem is defined as

the cognitive mechanism that people devise to self-assess their own value, as well as their confidence and pride.

The result of performing such self-evaluation generates affirmatory or dissenting opinions of the self that in turn contribute to enhance or diminish value. Additionally, this study proposes that the self-assessment individuals perform does not take place in a void but responds to the specific ordering of the social context and the interaction of individuals with the environment where they develop their activities. Thus, self-esteem is a constantly reshaping attribute associated with people's well-being and mental health.

Self-esteem plays a pivotal role in personal and academic achievement (Arshad et al., 2015; Fairclough, 2005; Mohammad, 2010; Rumbaugh et al., 2009; Zoller \& Gerard, 2011). Students with high self-esteem feel more comfortable with themselves and are therefore more efficient when dealing with schoolwork and school life in general. Students with high self-esteem also exhibit better skills in managing conflict of all kinds of situations at school.

A great deal of previous research on self-esteem has also supported the relationship between this construct and motivation. Motivation is an essential factor that explains the willingness of students to persist in achieving goals, the adoption of appropriate learning behaviors and the time student will devote to keep on task. Adequate motivation substantially augments levels of engagement and encourages students to adopt successful approaches to learning. Similarly, motivated students report showing lower 
levels of anxiety and exhibit healthier social skills that contribute to improving their performance at school (Abouserie, 1995; Basco \& Han, 2016; Zoabi, 2012). Having strong self-esteem is then a fundamental condition when considering students' progress and success, provided that possessing a high self-esteem facilitates learning. In accordance to its importance, self-esteem is equally vulnerable. Myriad factors have detrimental effects on students' self-esteem—including but not limited to underestimating their abilities, criticizing their appearance, or simply overlooking their worth with the consequent negative effects on students' academic and personal development. Students whose self-esteem has been undermined are less motivated to learn as their abilities to focus on their academic tasks decrease and they are not eager to take risks.

A large and growing body of literature is paying particular attention to issues associated with racial microaggressions and self-esteem. Thai et al. (2017) assessed Asian American emerging adults' perceptions of racial and ethnic microaggressions related to their self-esteem using the REMS scale offered by Nadal (2011) as reference. Thai and others also gauged the mechanisms that the target population adopts to navigate and survive an environment that conveys undermining messages against underrepresented populations. The adapted version of the perceived racial socialization scale (Tran \& Lee, 2010) was used to analyze this factor. As for individual and collective self-esteem indicators, Thai et al. (2017) used Rosenberg's (1965) and Luhtanen and Crocker's (1992) scales, respectively. In their study, Thai and colleagues (2017) concluded that Asian American emerging adults experience adverse reactions as a result of being exposed to racial microaggressions that diminish their psychological well-being 
and both individual and public self-esteem. In regard to the mechanisms that the target population adopts to deal with racial microaggressions, the study concluded that specific types of racial socialization messages might mediate the impact of microaggressions on self-esteem. Racial socialization entails the transmission of cultural traditions of battling oppression with actions that empower people to persist with dignity in a racialized environment. Results indicated that being prepared for bias partially moderated the relationship between microaggressions and both, individual and collective self-esteem. Thai et al. (2017) suggested that there is need to promote conversations about race within familial settings. These discussions are according to the authors useful to affirm selfesteem by enhancing bonds between members of a group.

Having a healthy self-esteem is not inherent to a specific social class, age, or race. However, some groups are more susceptible to developing lower self-esteem because of their disadvantaged position in society. Coming from a low-income family, belonging to an ethnically minority group, categorizing as a different cultural orientation, or identifying as LGBT may make people more vulnerable to experience discrimination in a variety of forms, either overt or covert (Golding et al., 1991; Lantz et al., 2005). Research has emphasized the profound cumulative effects of blurred hostilities on underrepresented people's self-esteem. These aggressions are not exclusive to a single place but permeate all kinds of environments and social interactions.

College settings can be threatening for underrepresented students as they may encounter a variety of adversities in their interactions with peers, teachers, or administrative. In most situations, underrepresented students are exposed to frequent hostilities that have a profound impact on their overall wellness. In looking at the 
relationship between racial microaggressions and psychological wellbeing of African American college students, Helm (2013) used the Racial and Ethnic Microaggressions Scale, Rosenberg's Self-Esteem Scale, and the Beck's Depression Inventory. The study reported evidence that racial intolerance is common to higher education institutions. Helm's study stated that African American students are exposed to everyday racial microaggressions in their institutions and that there is a multi-dimensional relationship between subtle racism and psychological well-being. Helm (2013) determined that racist events experienced by African American students were positively related to adverse effects on their self-esteem as well as on depression. Findings showcased how race is still a harmful major issue that underrepresented students face while pursuing a degree.

Additionally, Helm (2013) emphasized that because of their exposure to persistent microaggressions, African American students require spending additional time, energy, and emotional space trying to decipher and cope with the multiple effects microaggressions generate on their lives. In the case of African American students, contending with these hostilities produces layers of pain, frustration, and negative feelings. Sadly, students mostly assimilate their fate without any kind of support from their institutions. Helm's study recommended that higher education institutions start by acknowledging that the subtle manifestations of racism are existent and affect different student populations. Helm (2013) urged colleges and universities to take the agency to provide all students with more inclusive and safer educational environments.

The literature has identified the undeniable negative impact microaggressions have on weakening students' self-esteem (Harris et al., 2007; Tawa et al., 2012). Students with low self-esteem are more likely to have a poor image of themselves that translates 
into discouragement, poor academic performance, and unhealthy interactions. Nadal et al. (2014) studied microaggressions as a predictor of lower self-esteem as well as the types of microaggressions that most affect diverse groups' self-esteem. The study also looked at how these groups experience racial microaggressions. Latino (Hispanic, Latin, or Puerto Rican) and Asian (Pacific Islander or Filipino) college students participated in the study. Nadal's Racial and Ethnic Microaggressions Scale (REMS) and Rosenberg's SelfEsteem Scale (SES) provided data for the study. Evidence of the negative influence of microaggressions on students' self-esteem was manifest in the study. In regard to the types of microaggressions that are more likely to impact self-esteem, second-class citizenry or assumptions of criminality, and microaggressions that occur in school and workplace settings were the most frequent (Nadal et al., 2014, p. 468). Results demonstrated that those microaggressions that occur in educational settings and at workplaces are the most detrimental to students' self-worth. The study indicated that race is a predictor of microaggressions and that Black, Latina/o, Multiracial, and Asian American students, are the most frequent victims of hostilities. Furthermore, racial groups differ in the type of microaggressions they experience. Black and Latina/o students are usually ascribed as inferiors or coming from lower social classes, while Black and Latina/os students are perceived as criminals and second-class citizens. Exoticization is also common in Latinas/os because of their race, ethnicity, immigration, language, and accents, while Asian Americans are usually invisibilized in different spheres. Nadal et al. (2014) highlighted the need for institutions to identify the types of microaggressions students are exposed to as a step to prevent its occurrence. Since racial slurs are detrimental for students' self-esteem, Nadal et al. (2014) suggest accompanying 
students in building strong coping strategies that serve as a shield to protect themselves from developing low self-esteem. Finally, the study emphasizes on the importance of safeguarding self-esteem, as this is a protective factor against suicide and other disorders. The authors call for institutions that are more aware of the campus climate and the creation of programs that promote harmony and inclusion.

In analyzing microaggressions, Thai et al. (2017) and Nadal et al. (2014) relied on Rosenberg' Self-Esteem Scale. This 10-item scale has been widely used to measure global self-worth by assessing people's both positive and negative opinions about themselves (Blascovich \& Tomaka, 1993). In the 1960s, Rosenberg created the first version of the self-esteem measure. The scale has a reliability that ranges between .82 to .88 with a Cronbach's alpha ranges of .77 to .88 demonstrating to be both a unidimensional and two-factor (self-confidence and self-deprecation) scale. People of most ages can take the scale since there are no major differences in the reliability of the test for diverse groups such as adolescents, adults, people with physical or mental illnesses, drug users, or people with low literacy. The translations of the scale into different languages have not affected the reliability of the test to either gender or socioeconomic status (Louise, 2003). What makes this scale appropriate is the fact that it measures self-esteem as a global rather than as a multi-dimensional aspect. By using the Rosenberg scale, researchers can determine if subjects see themselves as worthy or unworthy. Individuals who see themselves as worthy do not consider themselves as superior; they acknowledge their limitations and expect to grow and improve according to their effort and work. On the other hand, people who see themselves as unworthy believe they do not deserve attention or respect, live in a constant self-contempt, and do 
not accept their limitations. The advantages of using Rosenberg's scale for this study are the simplicity of the language used, the number of questions, and the fact that it is available in different languages including Spanish.

Self-esteem is a crucial factor not limited to providing people with self-worth but also as a moderator between perceived discrimination and symptoms of poor mental health. Exhibiting a strong healthy self-esteem helps victims protect themselves against the dangers accompanying microaggressions. Umaña and Updegraff (2007) explored interrelations among discrimination, ethnic identity, cultural orientation, self-esteem, and depressive symptoms in Latino adolescents. Their study concluded that the higher level of self-esteem adolescents possessed, the better they were able to cope with manifestations of discrimination in their environment. Self-esteem then appeared to be a risk reducer operating as a protective mechanism of mental health. Other researchers (Mann et al., 2004; Rice et al., 1998) also analyzed the importance of self-esteem to mediate the negative effects of stress and depression support Umaña and Updegraff's conclusions. However, Umaña and Updegraff (2007) also noted that self-esteem only partially mediates the negative effects of discrimination. This finding suggested that discriminatory events still have a conclusive effect on adolescents despite they exhibit strong self-esteem. In the view that Umaña and Updegraff's study comprised US-born adolescents, the authors recommended the need of studies to determine the role of selfesteem as a risk reducer in other contexts and with different populations for these processes may vary considering factors such as setting, age, and origin.

Two main conclusions derived from the studies cited above. First, although findings from a range of studies suggest that underrepresented students experience low 
self-esteem and other signs associated with depression as a result of the constant exposure to discrimination, their needs in terms of providing them with safer educational environments are still overlooked. In conversations regarding underrepresented students' self-esteem, educators, therapists, and social workers still perceive the problem as a product of personality factors rather than the result of the exposure to constant hostilities that gradually undermine student's mental health and well-being (Tawa, et al., 2012; Umaña \& Updegraff 2007). Second, it seems necessary that institutions assure all students the best conditions for performing their activities in a safe environment despite their race, class, or social status. To do so, a more culturally informed evaluation of the racialized dynamics in educational settings should be performed to address concerns affecting identified at-risk groups.

\section{Microaggressions and Self-efficacy}

As human beings, we constantly face challenges in our lives. We want to feel that we are in control and that we will perform successfully towards our own realization despite obstacles. This realization is, undoubtedly a desire we all have. Self-efficacy plays a crucial role in both how we feel about ourselves and how we face situations toward achieving and succeeding in our goals in life. Self-efficacious people are able to recognize the goals they want to pursue, the aspects they need to change, and the desires they plan to fulfill (Schunk, 2012). However, the achievement of these visions comprises a series of actions that do not appear to be easy to perform since all demand people to believe in their capabilities to handle life circumstances.

Numerous definitions help us understand the concept of self-efficacy. The Cambridge English Dictionary (n.d) conceptualizes this construct as "a persons' belief 
that they can be successful when carrying out a particular task." Self-efficacy refers more to people's perception of being able to do things that result in the possibility of achieving a goal rather than it does to their real abilities. Bandura (1977) referred to self-efficacy as “the personal beliefs about one's capabilities for learning or performing actions at designated levels" (p. 2). This definition emphasizes that individuals use cognitive mechanisms to assess and identify their strengths that in turn will allow them to develop activities. Bandura (1977) also indicated that self-efficacy is a critical factor in promoting a sense of agency in people.

Ormrod, Anderman, and Anderman (2014) defined self-efficacy as “an impression that one is capable of performing in a certain manner or attaining particular goals” (p. 334). This perspective supports Bandura's view of self-esteem as a subjective thought that one has the skills to engage in such activities necessary to approach potential situations. The perception of one's ability to do something might not be necessarily precise, but it might have an effect on the extent and effort the person invests in achieving a goal. Erel (2000) defined self-efficacy as the "central persuasive belief about people's capabilities that they can control their own level of functioning and events that affect their lives" (p. 15). It can be inferred that, through performing deep analysis, people identify their abilities and, to a degree, regulate them so they can adequately perform in events occurring in their lives.

Erel (2000) conceptualized self-efficacy as the mechanism through which people play an internal subjective judgment of their abilities to perceive themselves reacting to different situations. The extent to which people trust their capabilities has an impact on every facet of their life, from their psychological state to behavioral manifestations that 
motivate them to strive to achieve goals. The above definitions concur in that self-esteem involves a conscious self-assessment of one's own capabilities when facing different situations. Once this self-assessment is performed, it results in understanding what we can do with our identified skills.

The study of the importance of self-efficacy dates back to 1977 with Bandura and his influential ideas on social cognitive psychology and social learning theories. Bandura asserted that self-efficacy has a pivotal influence on initiating and persisting in a behavior toward achieving a goal. Having the conviction of being effective defines people's decisions on even starting to cope with an event that seems threatening, and that will require extra effort to complete. In the same vein, Bandura identified self-efficacy as a determining factor in choosing the type of activities people will perform that might eventually result in succeeding in their endeavors. The expectations of successful attainment maintain people's engagement, thereby thwarting them toward persistence of spending more time and energy towards their goals. Thus, the stronger the perceived selfefficacy, the more active the efforts. Bandura argues that persistence in apparently risky situations that gradually become safe generates feelings of efficacy that ultimately remove defensive behaviors. People who go through this process successfully affirm their self-efficacy and are eager to engage again in new challenges. On the contrary, those who renounce striving weaken their expectations and retain feelings of failure and fear for more extended periods (Bandura, 1977). Accordingly, self-efficacy plays an essential function in the way objectives, tasks and difficulties are handled and regulated by people. Self-efficacy is not an innate trait. More specifically, people start building selfefficacy only at early ages and develop it throughout life. To shape self-efficacy, people 
engage in a complex process in cognitively assessing information from different sources (Schunk 2012). Bandura (1982) identified four sources that influence the formation of self-efficacy. The first is directly related to people's experience. A robust perception of efficacy derives from mastery experiences. In this sense, Bandura (1982) pointed out that the more successful people are at a specific domain, the more they develop a higher feeling of being efficacious at that domain. On the other hand, failure tends to weaken efficacy, especially when it has not yet been adequately ascertained. Bandura (1994) abounded on how this first source works in developing self-efficacy. Successful experiences that demand little effort do not contribute to cultivating strong self-efficacy but those that comprise some level of difficulty that demand individuals to sort some obstacles do. Bandura asserts that when individuals achieve their goals despite difficulties and prove themselves they are in control, their self-efficacy increases.

The second generator of self-efficacy is vicarious experience. Bandura (1994) claimed that observing people performing and being successful in their attempts supports people in assessing their efficacy. Following others of similar characteristics, age, or abilities thriving, generates feelings of confidence and encourages observers to venture themselves in achieving the success of their counterparts and vice versa. Social models then play a crucial role in shaping self-esteem. Bandura explained that individuals choose the models they want to learn from. In doing so, people try to find models who, in addition to being proficient, possess the willingness to persevere. Persistence in accomplishing a task despite impediments, interruptions, or delays in achieving success motivates observers to take risks seeking their own success. Models then, he says, not only serve as knowledge suppliers but as a source of extrinsic motivation for observers 
who eventually develop both skills and attitudes. Models as a source of extrinsic motivation, support others to stay motivated and on task.

The social environment also acts as a buffer of self-efficacy. A stimulating atmosphere is able to persuade people that they possess the abilities to prosper in an enterprise (Bandura, 1994). When people do not feel confident enough to perform an activity, persuasion from a peer helps them rely more on their skills. Receiving positive assurance form others has a multiple-fold effect. Persuasion supports individuals to deal with their lack of confidence in performing a task and keeps people on duty because they want to please those who trust them (Burgoon, 1981). However, Bandura (1995) explained that in the day-to-day relationships, people also receive negative persuasive messages and that they are highly susceptible to these kinds of adverse messages. Individuals' receiving constant invalidations about their capabilities has a detrimental effect. People convinced that they are not able to perform a task are not eager to engage in challenging activities and, if even they were, he says, their determination to achieve the goal decreases significantly.

The last element influencing self-efficacy is people's own somatic and emotional states in assessing their abilities (Bandura, 1995). People react to stressful situations with a high degree of tension that provokes physical symptoms like sweats, heartbeat increase, and pains. For people with high self-efficacy, this arousal of stress gives them more dynamism to face the situation. On the contrary, those with low self-efficacy find themselves paralyzed and unable to take action. The emotional state also serves as a buffer to judge self-efficacy. People with healthy self-efficacy maintain a positive attitude towards adversities and believe that with effort and patience they will be able to succeed. 
According to Bandura, people with weak self-efficacy tend to persist in a negative mood and see misfortunes as impossible to surmount.

These considerations imply that forming beliefs of personal efficacy is a complex process of self-appraisal, which entails selecting, weighing, and integrating information from multiple sources. In this appraisal process, the social environment where individuals perform their activities plays its influential role. Culture and the context affect not only the type of information provided by the various sources, but also which information is selected and how it is weighed and integrated into people's self-efficacy judgments (Schunk, 2012). Thus, the importance of a positive environment where people are valued and receive constant encouragement is crucial to enhance their self-efficacy.

Zimmerman (1995) discussed the features of academic self-efficacy. The first indicator encompasses students' assessment of their own aptitudes to develop activities. In doing this assessment, Zimmerman (1995) notes that students look at the abilities they possess to fulfill the task demands, despite personal qualities as physical or psychological traits - that is to say that they perceive their capabilities on how they feel about themselves in general.

A second feature deals with self-efficacy multidimensionality. Rodgers, Wilson, Hall, Fraser, and Murray (2008) showed how students conduct analysis to determine what areas, domains or activities they are more productive at, meaning that they know what their strengths and weaknesses are and perform accordingly. For instance, whereas a student is good at Science, such does not necessarily mean that they are good at literature. The student is conscious of their potential in science and acknowledges their deficiencies in literature. Self-efficacy is also multidimensional in the view that being self-efficacious 
at a particular domain implies the command of a subset of skills and knowledge that operate as pillars of that specific domain.

Additionally, the theory suggests that self-efficacy is highly context dependent. This third characteristic is of particular interest for this study because while performing academic activities, students receive the influence of their context that in turn has an impact on their self-efficacy beliefs. Bandura (1994) stated that, besides instruction, an array of social factors have a positive or negative effect on students' judgments of their efficacy such as type and quality of peer modeling, comparisons students establish with their counterparts, motivational enhancement they receive from teachers and peers, relationships they create with peers, and teachers' interpretations of students' performance. The last component of self-efficacy involves the dependence on a proficiency criterion of performance rather than a normative. In other words, students perceive their skills in reference to the level of difficulty a given task entails rather than on the comparison between their skills and those of their peers (Zimmerman, 1995).

Throughout the years, self-efficacy has received attention from a variety of fields. The interest resides in the established association of self-efficacy with multiple human spheres, especially those related to behavior, affection, motivation, and performance (Williams \& Rhodes 2014). Self-efficacy is especially germane to school learning and other achievement situations (Schunk, 2012). Although self-efficacy is not necessarily a determinant factor of academic achievement, it converges to an array of aspects such as developing deeper interest in activities, forming stronger bonds, and recovering easily from setbacks that make students' experience gratifying and rewarding. Nichols (2007) provides an example of how self-efficacy influences students' better adjustment to 
college. Nichols examined first-generation students' self-efficacy related to their academic performance. Results suggested that, although self-efficacy has a moderate effect on academic achievement, it is a powerful determinant on college adaptation. Students who felt confident of themselves, not only exhibited better academic performance but more importantly adjusted better to college, established bonds with their institutions, were more optimistic on their ability to overcome difficulties and gave more effort on their duties. These students were more likely to persist in achieving their goals and demonstrated higher levels of commitment to their work. On the contrary, first generation students with low self-efficacy were more prone to giving up when facing obstacles and not showing enough strength to perform their tasks. Nichols (2007) concluded that self-efficacy is a predictor of persistence in college with long-term effects on college students.

Linnenbrink and Pintrich (2003) discussed the relationship between self-efficacy, students' engagement and learning in the classroom. The authors identified three types of relationships between self-efficacy and engagement, namely behavioral, cognitive and motivational relationships. Students with high self-efficacy exhibit easily observable behaviors of engagement such as effort, persistence, and help seeking. They stayed on task longer and did not easily resign from their goals. Conversely, those with low selfefficacy were skeptical about their skills to succeed and were more prone to abandon their schoolwork when they faced difficulties. Likewise, the quantity and quality of effort students with low self-efficacy payed to their tasks were weaker compared to their counterparts who exhibited high self-efficacy. Interestingly, self-efficacy was a stronger predictor of persistence than students' knowledge. Students with sufficient prior 
experience but with low self-efficacy were not confident that their skills were appropriate to achieve a task. Thus, they were less likely to embark on an assignment that seemed challenging (Linnenbrink \& Pintrich, 2003).

Another behavioral aspect associated with self-efficacy is learned helplessness. Students with low self-efficacy perceived themselves as unable to regulate their own behavior and believed there was no association between their response and an expected outcome. In other words, these students assumed that no matter their efforts, the result would always be adverse. Conversely, students with high self-efficacy considered that they were able to control some situations in their learning environment and that hard work would always result in achieving success. Another indicator of behavioral engagement is help seeking. In their study, Linnenbrink and Pintrich (2003) demonstrated that self-efficacy related to help seeking. However, looking for help was not an option for students with low self-efficacy as they thought that asking for assistance put them at a disadvantage in the view of their teachers and peers. Students who did not see their counterparts or teachers as potential supporters but as detractors of their work, missed the opportunity to get extra support that ultimately generated more frustration and despair (Linnenbrink \& Pintrich, 2003).

The same study established the relationship between self-efficacy and cognitive engagement expressed as the quantity/quality of action exerted by students as pivotal. Staying cognitively engaged was sometimes tough for students, as it demanded that they put forth extra effort to accomplish the learning task. Self-efficacy was associated with the use of more in-depth processing strategies and cognitive engagement to learning. Students with strong self-efficacy were more inclined to look for strategies that facilitated 
their learning. These students were aware of their thoughts, emotions, and behavior that allowed them to face difficult situations more rationally (Linnenbrink \& Pintrich, 2003). These findings supported Schunk and Mullen (2012) assertions that self-efficacious students are permanently reflecting on their performance; they set attainable goals and evaluate their progress throughout the process. Another characteristic that cognitively motivated learners exhibit is that they are able to choose learning strategies that apply for their specific needs and avoid distractions. These learners are productive in the sense that they manage their time correctly, look for best learning environments and are not afraid to seek help when necessary. During their learning, students with strong self-efficacy are continually monitoring their progress to make sure they are on the right track and to make necessary adjustments when required (Schunk \& Mullen, 2012).

Self-efficacy also connects to motivational aspects such as students' personal interest and values. Although it is not clear if motivation sparks personal interest or if it is interest that generates motivation, what is clear is that there is a close connection between both constructs. In general, students who exhibit high levels of self-efficacy, experience positive emotions that keep them engaged with their school and schoolwork such as pride, happiness or excitement. In contrast, those with low self-efficacy feel negative emotions regarding school and their work that make them anxious or depressed (Linnenbrink and Pintrich 2003). Dogan (2015) supported the relationship between selfefficacy and motivation. Dogan looked at the association of student engagement, selfefficacy and academic motivation on academic performance. Conclusions suggested a strong correlation between academic self-efficacy and academic motivation on students' performance, meaning that self-efficacious students are eager to perform academically to 
the best of their capabilities and motivate themselves to learn. Altogether, self-efficacy is crucial to ensuring students' engagement in their academic work since students who feel confident in their own abilities are more likely engage in terms of their behavior, cognition, and motivation. As stated at the beginning of this analysis, self-efficacy is not an innate fixed characteristic but a malleable trait that can strengthen when contextual features are favorable. In this light, teachers and institutions play a role in influencing students' self-efficacy perceptions that contribute to their academic success.

Self-efficacy is susceptible to environmental factors. Smith (2017) examined the relationships among race, racial microaggressions, academic motivation, and academic self-efficacy. Founded on the evidence that racial microaggressions are frequent in the classroom, the study demonstrated that the higher degree of microaggressions resulted in a more substantial impact on students' self-efficacy. Smith's research showed that the intensity of microaggressions depends on race with African American students as the most frequent targets. The study indicated that African American students who experience high levels of microaggressions displayed the lowest levels of self-efficacy compared to their Asian American, Hispanic and White counterparts. Smith (2017) used the Racial Microaggressions Scale (RMAS), this is a scale that mirrors Sue and colleagues microaggressions taxonomy different from the one proposed by Nadal, the Academic Motivation Scale (AMS) and the College Academic Self-Efficacy Scale (CASES) to measure the different constructs of the study. The results suggested that selfefficacy for the case of students of color was predicted by invisibility and environment. Students of color perceived that they were not meaningful contributors to the class, thus invisible and not prominent. Smith (2017) concluded that students' self-esteem remained 
low in environments where their academic worth was not valued or enhanced.

Conclusions from this study attributed schools a prominent role in the personal and academic development of their students. Educational institutions should thrive towards the holistic realization of students despite race or social status. As for implications of this study, Smith (2017) stated that schools failing to provide a nurturing environment for all students where their self-efficacy as a component of academic success was not enhanced, augmented the gaps between marginalized and privileged populations. Some steps toward reducing gaps, according to the author, were augmenting the representation of people of color in their institutions, developing awareness on issues related to diversity, and identifying challenges students at risk may have with their motivation. As for further research, Smith (2017) recommended examining the relationships between microaggressions and academic self-efficacy, as well as assessing the nuances this phenomenon may take in variables such as gender and socioeconomic status.

Students of color and minorities experience unique challenges in college. McCullagh (2007) looked at the role of racial microaggressions on college students' selfefficacy, academic integration, and persistence in predominantly White institutions. Participants in this study took three different surveys to determine the constructs under analysis: REMS, Persistence/Voluntary Dropout Decisions Scale (P/VDDS) and College Self-efficacy Inventory (CSEI). Surprisingly, the study did not report an association between microaggressions and self-efficacy, social integration, academic integration, or persistence in students of color, which according to the author contradicted most of the previous research done in this respect. However, McCullagh (2007) presumed that this contradiction might derive from different reasons such as participants already possessing 
skills to handle discriminatory experiences or the inadequacy of the REMS to capture all the variables associated to microaggressions. The fact that only one out of the six scales of the REMS inquires about school and workplace-related microaggressions is a limitation to capture students' experiences. McCullagh's assumption that the REMS was not a powerful instrument to measure all the manifestations and impact of microaggressions indicated that other type of methodologies could be adequate to study this phenomenon. In this regard, Wong, Derthick, David, Saw, and Okazaki (2014) highlighted the limitations of quantitative research when looking at sensitive and complex issues such as microaggressions. Wong et al. (2013) identified some of the reasons for such limitations as the inability of self-reported scales to capture and determine the effects of microaggressions.

Smith (2017) and McCullagh (2007) used quantitative approaches to their analysis. In these studies, self-efficacy was measured with the College Academic SelfEfficacy Scale (CASES) and College Self-efficacy Inventory (CSEI) correspondingly. For the purpose of the present study, Schwartzer and Jerusalem's (1995) General SelfEfficacy Scale (GSE) will be used. The ten items of the GSE are unidirectional and have been used in several studies to measure perceived self-efficacy in different countries and all kind of populations. The instrument has been shown to have high levels of reliability with Cronbach's ranges from .76 to .90 . Another reason for using this scale is that its short version has been adapted to several languages including Spanish.

According to Wong et al. (2013), naturalistic approaches help researchers to understand microaggressions thoroughly. These inquiry methodologies assist in having a more thorough consideration of the cumulative effect of racial microaggressions. 
Qualitative approaches have also contributed to explaining how racial hostilities affect students' self-efficacy. Crispen (2017) used qualitative methods to examine the experience of underrepresented college students with racism, microaggressions, campus climate, and how these elements influence students' self-efficacy. Despite Crispen's acknowledging that the qualitative survey used in the study was not enough to capture students' experience in a more detailed fashion, some valuable conclusions were drawn. A population of African/biracial, Hispanic/Latino, Asian and White students participated in the study. Results suggested that all the African American students who completed the qualitative survey reported having witnessed or experienced racism on campus. Despite the prevalence of microaggressions in this population, many respondents indicated that they would not report the events because they were afraid of the repercussions of doing so or because they believed the events lacked enough importance to be reported. In terms of self-efficacy, Crispen stated that underrepresented students doubted their ability to succeed in college although some of them were top students. In terms of campus climate, Crispen's study suggested that having friends, belonging to a social group that provides support, teachers, allies, and staff are decisive elements in helping students succeed in their academic pursuits. Conclusions of this study showed concern in the sense that targets do not perceive microaggressions in their real dimension. Underrepresented students do not understand the pervasive problem affecting them; the reality is that microaggressions challenge their sense of belonging to the institution and their abilities to perform academically.

Self-efficacy is associated to students' learning in terms of their behavioral, cognitive and motivational engagement in the classroom. It also links to students' 
persistence and how they develop their academic activities in their institutions. Different factors have a detrimental effect on students' self-efficacy. Racial hostilities as a source of emotional abuse and distress against minorities have the power to undermine students' self-efficacy. Effects of hostilities are so severe that students believe that failure to attain goals is because of their personal shortages or inabilities rather than the inequities of society. These studies give self-efficacy a paramount importance in students' development. They also support the idea that institutions need to give closer attention to manifestations of prejudice that can affect students and urge them to continuously monitor and adjust their climates for the benefit of at risk populations.

\section{Responses to Microaggressions}

Exploring microaggressions is not limited to determining the types of racial offenses people experience in their lives. In the view of the harm that racial microaggressions cause on people's lives, another important aspect to consider is the responses or reactions indignities generate. A popular motto from Maya Angelou says "people easily forget what others say and do but they hardly forget how others make them feel." Research shows racial hostility, dismissiveness, invisibility, stereotyping and subjugation take a toll on how people about themselves and consequently on their mental health (Sue et al., 2007). Unfortunately, manifestations of discrimination by virtue of race are pervasive to all kind of settings, and higher education is not the exception. To this end, Chesler and Crowfoot (1989), maintain that racism is so engrained in colleges and universities that at times, it is difficult to recognize. However, that racial frictions between people belonging to different groups are hard to see does not mean they do not provoke a profound effect on victims. Crowfoot (1989) maintains that as soon as a racial 
hostility occurs, victims incorporate it to their experience and intentionally or involuntarily generate a response.

What targets of racial microaggressions experience when hostilities happen is explained by Sue et al. (2007) as a series of events that affect their people's energy and emotional health. Immediately after a hostility takes place, targets start formulating a number of questions that help them clarify the event. Such questions start with asking themselves about the real occurrence of the hostility and continue with them determining if the event was intended or accidental, considering the right response according to the circumstance, finding the suitable evidence to prove the case, and deciding if reacting to the incident is worthwhile (Sue et al., 2007). All this process of questioning takes place in a short span resulting in victims feeling completely drained and overwhelmed.

Once targets have undergone the multiple questions that strike their minds, three possible internal courses of action might emerge. Sue et al. (2007) suggest people first try to determine the incidence of the microaggression. To do this, people turn to their previous experience with similar events. This exercise is "contextual in nature and involves life experiences from a variety of situations" (p. 279). The purpose of this internal operation is to find similarities with other events and as Sue et al. (2007) explain, people intend to connect episodes. In most of the cases, they conclude, people experiencing microaggressions, despite of the setting or source, come to the conclusion that the only condition that triggers the biased hostile event is the color of their skin.

A second stage comprises the nature of the reaction the person generates that has effects both on the perpetrator and the victim. The most frequent option is to sit back and do nothing concerning the hostility. Several reasons explain this behavior. It is possible 
that the targets cannot clearly define if the hostility happened. It might also happen that the victims are at a loss to providing a response. They may also be afraid of the costs their reaction might produce. Similarly, victims may conclude that reacting to the hostility neither contributes to improve the situation nor changes the perspectives of the offenders. Finally, victims try to convince themselves that the event did not occur and, therefore, they continue their lives (Sue et al., 2007). Although all these alternatives are plausible for the victim to avoid reacting, they entail a high emotional and psychological cost because, as Sue et al. (2007) assert, “it may mean a denial of one's experiential reality, dealing with a loss of integrity, or experiencing pent-up and frustration likely to take psychological and physical tolls" (p. 279).

A final potential response entails replying to the offense with anger, which despite being the healthiest reaction in terms of reducing targets' frustration, provokes the most adverse outcomes for victims. Given microaggressions are typically underestimated, an outburst might result in people accusing the victim of being oversensitive about race and racism. Reacting with anger also leads to being judged as unreasonable, aggressive, unfriendly, and antagonistic, which ultimately results in augmenting the conflict between people (Sue et al., 2007). In sum, Sue et al., (2007) conclude that a healthy psychological reaction from the victim in the form of an angry reply, does not contribute to stopping hostilities in the racialized environment; conversely, it exacerbates the animosity between the parties. The limited options targets of racism have to react to, as well as the negative consequences they produce, trap people in what Sue et al., 2007 call "damned if you do, and damned if you don't”' (p. 279). In any case, Solórzano et al., (2010) offer advice regarding the importance of extending research on campus racial climate that contributes 
to giving voice to underrepresented students, getting a deeper understanding of the effects of racial microaggressions, and examining the responses to racism.

\section{Coping with Microaggressions}

As discussed earlier, every microaggressive event involves a response where victims are restricted to deciding between two options: remaining silent and swallowing the incident, or reacting with anger. Regardless of the reaction, people also engage in looking for suitable alternatives that help them cope with microaggressions. Sue et al. (2007) recommend that because racial hostilities have repercussions on mental health, looking into the mechanisms people use to mitigate those effects is advisable. By the same token, the authors consider that a deeper understanding of coping strategies is necessary to guide students in the adoption of the best approach according to the characteristics of their context.

In psychology, coping refers to "the use of cognitive and behavioral strategies to manage the demands of a situation when these are appraised as taxing or exceeding one's resources, or to reduce the negative emotions and conflict caused by stress" (VandenBos, 2015, p. 252). Coping is innate to human beings and denotes the actions people take to safe ward themselves from any type of psychological harm that causes pressure or tension (Pearlin \& Schooler, 1978).

Coping behaviors are triggered by a situation that individuals want to solve because it increases their levels of stress. Targets of microaggressions experience psychological, physiological, and behavioral stress as a consequence of their exposure to racial hostilities (Franklinet al., 2014; Pizarro \& Kohli, 2018). In order to alleviate the 
stress caused by hostilities, individuals engage in various coping strategies that can be adaptive or maladaptive (Holder, Jackson, \& Ponterotto, 2015).

Decuir-Gunby et al. (2019) state that individuals using adaptive coping strategies address stressful situations in positive manners. Adaptive strategies not only reduce stress but also result in healthy outcomes for people. In dealing with racial stressors, people adopt problem-solving behaviors, look for emotional support, and try to reframe-reassess the stressful situations cognitively. The authors provide examples of adaptive strategies people use including: engaging in conversations with people experiencing similar situations, communicating their emotions to others, confronting racism, forming networks with other people in similar situation, looking for appropriate guidance or mentorship, seeking religious support, establishing boundaries to protect themselves, and engaging in self-care actions (DeCuir-Gunby et al., 2019). Other adaptive actions mentioned by the authors include meditating, exercising, playing an instrument, going shopping, reading, spending time with friends, and enjoying nature. People using any of the aforementioned strategies employ their knowledge and internal strength to deal with negative situations. In turn, these actions prevent them from negative reactions that may result in augmenting their stress.

DeCuir-Gunby et al. (2019) emphasize that despite the benefits of using adaptive strategies to microaggressions, people usually involve themselves in maladaptive actions in their attempt to physically, emotionally, and mentally remove the stressors. However, these coping strategies result in negative consequences that do not help to reduce the stress but to increase it. Maladaptive coping strategies result in people: suppressing or internalizing their feelings (social isolation, self-criticism), developing physiological 
effects (high blood pressure), experiencing loneliness, and refusing to confront racial hostilities. Other maladaptive strategies are oversleeping, procrastinating, leaving, detaching, eating unhealthily, drinking alcohol, taking revenge, saying hurtful things, being hostile, and lying. By using maladaptive strategies, people intend to deny racialized hostilities around them. This denial has an effect on their emotions that can cause severe anxiety disorders (DeCuir-Gunby et al., 2019).

The study of coping is crucial in the way that it is a mediator between stress suffered by targets and their mental health. Research has reported various approaches victims of racialized hostilities adopt to reduce stress and harmful consequences derived from such aggressions. Lowe et al. (2012) used qualitative inquiry to analyze racism trauma, coping and the implications for victims of racism. Interviews from people of different ethnicities led to recognize some strategies they use to cope racial hostilities. In the study, interviewees asserted that a suitable approach to cope with racism is to maintain themselves in a constant state of "denial." People believe that by avoiding the constant recall of aggressive events, they are able to reduce harmful outcomes of racism. However, although people consider denial as a good coping strategy, the internal suffering it causes and the implications on their mental and emotional health are, to say the least, disturbing.

Silence is another strategy people adopt as a coping strategy (Lowe et al., 2012). Remaining silent after a hostile event reported to be very overwhelming for victims who would continue to experience negative feelings after several years the aggression occurred. The story of a participant in their study highlighted an episode where she openly reacted against a racist comment. Her reaction provoked an argument with the 
interlocutor, resulting in the victim experiencing depression and even guilt for having felt shame of her identity and cultural background. Evidently, when targets deny or remain silent, they are unable to address the racist slurs properly producing the accumulation of harmful feelings with unpredictable consequences. Similar results appear with those who react. They tend to feel embarrassed by their reactions or remorseful for having initiated a turmoil that did not take them to solve the racial issue. An interesting point raised by Lowe, Okubo, and Reilly (2012) was that, in looking for some relief to their emotions, people also confide their experiences in someone who has lived similar hostile situations because they understand how being attacked feels and are therefore able to provide better support. Common emotions people experienced in the stories depicted by Lowe, Okubo, and Reilly (2012) were loneliness, powerlessness, frustration, anger, devastation, confusion, melancholy, dismissiveness, and of course a sense of loss. The study recommended providing effective support to people exposed to microaggressions so they can deal with the multiple consequences racism leaves on their lives.

The literature also offers confirmation of racialized stress among faculty and how they cope with stressors. Salazar (2009) looked at strategies faculty of color use to survive and thrive in academia. This qualitative study analyzed responses of seventeen faculty on the strategies they used to navigate day-to-day and longer-term academic life. Results suggested that although campus climate was to some degree "harmless," faculty believed their institutions struggled to put into effect an adequate language of diversity. They believed that in their environment, there were still traces of segregation and prejudice. Regarding the climate at higher education institutions, some faculty recognized the presence of new racism, institutionalized racism, and inconsistencies between what is 
said about addressing racism and actual practice. Interestingly, participants in this study experienced what Sue et al. (2007) described as the Catch-22 effect to responding to microaggressions, meaning that faculty went through a difficult process of "internal struggle of decoding experience, deciding not to respond, weighing the consequences, and expending effort to respond to microaggressions" (Salazar, 2009, p. 194). In Salazar's study, coping is described as survival approaches faculty adopt to deal with the detrimental outcomes of microaggressions. Such strategies included protecting self from harm, finding allies, shielding aspects of self from scrutiny or attack, affirming one's self sense of selfhood as a racial/cultural being and validating one's perceptions.

Drywater-Whitekiller (2017) analyzed Native Americans experiencing and coping with racial microaggressions through narrative. Nineteen Native American students pursuing an undergraduate degree participated in the study. Students came from three federally-funded higher education institutions in the Midwest and Southwestern part of the United States. The study identified four types of microaggressions against Native Americans, namely alien in one's own land, identity scrutiny (you do not look Indian), second-class citizenry, and invalidation of Indigenous ways of knowing. Coping analysis was carried out in reference to the alien in one's in own land category. Findings suggested that, for the most part, Native American students used resilient coping mechanisms in terms of enhancing their bonds to the land. From Indigenous students' perspective, they belong to the land. Participants said that their land marks the spiritual connection they have with the world as it maintains the bond to their ancestral heritage: Land for indigenous people, they said, is the "home" and the "uniqueness of being" that ultimately creates their collective identity. The land also provides Indigenous people with 
a different basis of education pathways and thinking that does not pertain to the development of intellect but to the understanding of themselves. Because Indigenous communities were forced to abandon their land, the people became the source of their connection to the land. This explains the fact that for Indigenous students, the place where their people live is their land no matter the location. Home, as they call it, is the place where they feel safe, valued and understood. In other words, their people, their

home - these are the sources of their resilience. It is at home where they are able to face environmental hostilities. Home is the place where they can make meaning of their culture and identity (Drywater-Whitekiller, 2017). Participants in this study adopted adaptive coping strategies by identifying in each microaggression they received, an opportunity to reinforce their Native identity, a space to evolve through a process of personal reassessment in an effort to "restore their balance of who they believe they are as Native Americans" (Drywater-Whitekiller, 2017, p. 168). Results of this study also suggested that the identity of Native American students, created in a spiral fashion of intertwined overlapping circular patterns, took them to constant growth and renewal. Drywater-Whitekiller concluded that for Native American students, "moving on to more positive things reflects the ability to return and re-engage in spite of obstacles, and this is the foundation of cultural resilience" (p. 170).

\section{Indigenous Populations, Schooling, and Microaggressions}

\section{in the Americas and other Countries}

As a new field of study, racial microaggressions have gained attention over the past few years. Abundant research on microaggressions has been carried out in the United States, analyzing African-American, Asian-American, Latina/o people, and people with 
disabilities. The contributions of research in US contexts have sparked the interest of understanding the presence of this social phenomenon in other countries. To narrow the focus of this study, there is a need to look at the literature that describes how microaggressions manifest in indigenous populations outside the United States.

Canel and Yohani (2019) studied whether microaggressions were still present in the Canadian context. The authors validated their study acknowledging that despite Canadian universities are responsive to make indigenous education and worldviews an essential component of education; there are still gaps in the understanding of how this population navigates Canadian institutions. Canel and Yohani (2019) asserted that attention has been paid to institutional racism exerted against the Indigenous community, but little attention has been given to subjective experiences touching this population. This qualitative study used focus groups and follow-up interviews to collect data from seven participants. Experiences of interviewees led to the identification of seven domains of microaggressions as overt discrimination, the assumption of intellectual inferiority, the assumption of criminality, invalidation or denial, the concept of the second-class citizen, racial segregation, and the myth of meritocracy (Canel and Yohani, 2019). Traces of overt discrimination mentioned by participants included mockery, name-calling, physical violence, and exclusion from education that caused feelings of anger, confusion, and sadness. However, students reported more exposure to subtle types of discrimination. Canadian aboriginal students described being deemed as intellectually inferior and feeling constant academic pressure that negatively influenced their self-efficacy. Doubts about their integrity and honesty were also common events aboriginal students faced to the extent that they worked harder to prove to people that they were not criminals or gang 
members. Aboriginal students' history, culture, identity, and racial reality were invalidated in the Canadian educational context. Additionally, being ascribed as secondclass citizens was also a theme that emerged throughout the interviews. Students reported having been avoided, ignored, rejected or treated differently when requesting a service for the sake of their looks. Societies create implicit barriers between people because of their race or social condition. Canadian aboriginal students perceived these inherent obstacles as unwritten racial segregation rules everybody follows but denies at the same time. Privileges received by aboriginal students in the form of scholarships and other benefits were likewise seen as undeserved by their counterparts. White students were constantly sending messages accusing Indigenous students of abusing the system (Canel and Yohani, 2019).

Canel and Yohani (2019) concluded that, in the Canadian educational context, Indigenous students still suffer from racial discrimination in terms of racial microaggressions. A remarkable aspect of this study was that Canadian aboriginal students were highly aware of racial issues affecting them. This awareness of the racialized environment is pivotal because it is the first step victims take to contest hostilities. Evidence from this study suggested that Canadian higher education does not have enough preparation to tackle racial issues. Canel and Yohani's study is an example where a heavy load of microaggressions is still rampant against underrepresented students in Canada. The study called for giving more attention to the numerous aspects that need further exploration like the association between systemic racism and microaggressions, as well as the analysis of microaggressions with other groups in Canada. 
Several studies in Canadian educational contexts have analyzed Indigenous students' experience with racism and how it influences their academic achievement. These studies have revealed disparities in terms of school completion and academic performance between Indigenous and White populations. Although statistics on completion and academic performance are essential for knowing the situation of Indigenous students in Canada, Bailey (2016) proposed the need of an in-depth analysis that informs how anti-Indigenous racism in Canadian higher education contexts manifests. Bailey conducted one-to-one semistructured interviews with Indigenous and non-Indigenous students in an effort to examine their experiences and perceptions about racism issues in university settings. Several themes emerged from the interviews. Overall, the analysis revealed low interaction levels between Indigenous and non-Indigenous students indicating that there is still unbeatable racial barriers that impede adequate social interactions. As for perceptions and forms of racism identified by students, Bailey (2016) concluded that views depend on whether students were indigenous or non-indigenous. The non-Indigenous students in this study perceived campus environment as welcoming to all students while Indigenous described the environment as not necessarily hospitable for Native people. In terms of actions involving racism or discrimination, students described events as covert rather than overt. Another thread in Baily's study dealt with the audience. Both Indigenous and non-Indigenous students would be extra careful when talking about racial issues or disclosing identity. Discussing race depended largely on the audience they were addressing. In fact, in-class experiences with racism were also described in Bailey's study. The majority of Indigenous and non-Indigenous students reported having witnessed at least one episode of racism in the classroom. Bailey raised 
concerns about the university system, challenging it as responsible for creating barriers that affect Indigenous students. Lack of program opportunities for Indigenous studies, resistance to change from administration, and minimization of Indigenous students' concerns were the most common issues identified by the researcher. A final thread theme identified in Bailey was the feeling of academic and social isolation that Indigenous students said they experience in their institution. As a closing remark, Bailey promoted the need for more research in different contexts to gauge how institutions put in place practices that address Indigenous populations' needs.

In Latin America, there are more than five hundred Indigenous groups. Starting from Patagonia in Argentina to northern Mexico, the account for Indigenous people is about 42 million, which represents approximately $7.8 \%$ of Latin America's population (World Bank, 2015). Although the onset of the millennium brought considerable improvement to all countries in Latin America, with substantial reduction of poverty and progress in the access to services and common goods, this growth was not proportionate to all population. World Bank (2015) stated that Indigenous people did not receive much of the benefit from the "golden decade" and that they still suffer from the consequences of poverty and marginalization. Poverty in Latin America touches $43 \%$ of the Indigenous population, which doubles the percentage of poverty of non-Indigenous people (World Bank, 2015).

Although education as an indicator of progress and social mobility has seen development over the last decade in Latin America, this advancement has not necessarily meant the reduction of disparities especially in the quality of education provided to people living in poverty and at-risk populations (World Bank, 2015). The World Bank 
report highlighted the need for education systems to improve the inclusion of indigenous people in Latin America as the only option to turn things around in the region. The report called for an education that does not concentrate merely on the numbers but in assuring cultural pertinence where biases, prejudice, and discrimination do not affect Indigenous students' academic endeavors.

Regardless of apparent improvements in Latin American education, the presence of Indigenous students in academic environments still represents challenges to them as they are often subject to discrimination. Chavez (2008) stated that although in Mexico ethnicity is not necessarily a factor influencing academic failure, Indigenous students pursuing a university degree face adverse conditions such as isolation and discrimination. Chavez (2008) asserted that admission to higher education in Mexico is open to all kinds of students despite social status or ethnicity and that the number of Indigenous students in universities has increased in recent decades. In fact, some institutions, especially those located in areas where the Indigenous population is high, offer Indigenous students support that includes scholarships and counseling services. The author highlighted that, regardless of diversity in Mexican universities, the experiences of Indigenous students while in these institutions have not been addressed. In examining the opinions of Indigenous students in a Mexican university, Chavez (2008) determined that they undergo two types of challenges: personal and academic, being personal conflicts the most difficult to surmount (Chavez, 2008). According to the author, personal struggles have profound repercussions in academic achievement and dropout rates. Chavez (2008) determined that solitude is the common obstacle Indigenous students experience since their being accepted in already established groups of non-Indigenous students is a real 
challenge. To survive loneliness, students usually look for peers belonging to their same ethnicity in whom they find acceptance and mutual care. More importantly, Indigenous students adopt mechanisms that drive them to persevere in achieving their goals such as continually reminding themselves of their parents' struggles to afford their studies and the possibility to help them overcome poverty in the future (Chavez, 2008). In a concluding remark, Chavez (2008) stressed that ethnicity was the main reason for Indigenous students' feelings of discrimination and that they opted for isolating from their peers as a mechanism to protect themselves against hostilities.

One of the most straightforward mechanisms to assess inequities in society is to look at the educational system. Martinez and De la Torre (2010) asserted that in the "school system in Ecuador as a whole, we find a segregated, closed, and hierarchical structure that faithfully reflects Ecuadorian society, perhaps one of the most segregated in Latin America" (p. 19). This claim depicts the undeniable reality of Ecuadorian education. In all levels, institutions do not guarantee a welcoming and healthy environment for students. In fact, it is at the school where students especially those coming from low-income families or ethnic minorities start experiencing their unbeatable disadvantages. For many Indigenous students, the school setting is the source of discrimination that has become so ingrained that they assume it to be usual (Martinez and De la Torre, 2010). Despite this reality, there is a paucity of studies discussing issues related to race.

The work of Hollenstein (2008) pioneered an innovative approach to examining microaggressions and provided valuable insight into the nuances of this phenomenon in the Ecuadorian education system. Hollenstein (2008) proposed a model for the analysis 
of racial microaggressions. The model comprised two steps. The first involved two elements: the trigger (conflict situation) and symbolic resources (racial markers). The occurrence of both components results in the microaggressive act where the perpetrator plays a key role. In a second step, the victim's response to the microaggressive act takes a central role. In this second phase, Hollenstein's model also examined the responses given by perpetrators, authorities, and witnesses.

Hollenstein (2008) applied this model to analyze the course of daily racial dominance and contesting. The stories of discrimination suffered by three indigenous women while in primary and secondary school illustrated the nuances of racialized school environments where indigenous people interact with White-Mestizos.

Hollenstein (2008) analyzed three aspects: racial hostilities (perpetrators and symbolic resources), responses given by victims, and the emotional impact of microaggressions. The author determined that racial hostilities were almost the same all over the country but with different nuances depending on the ethnic characteristics of the school targets attended. Hostilities also resulted from different racial rituals where Indigenous women suffered the intersection of gender and race. In describing hostilities in the school setting, Hollenstein (2008) described discriminatory acts as sporadic and unpredictable, happening inside or outside the classroom. In relation to perpetrators, they were usually female students attacking their Indigenous female counterparts. Teachers were also perpetrators whose hostilities were spontaneous in assuming that Indigenous students were inferior when compared to their peers. The most common racial symbolic resources determined by Hollenstein were racial markers such as skin color, outfit, language, hair, poverty, and racial myths such as assuming Indigenous students are dirty 
or inclined to steal and tell lies. The responses identified by Hollenstein were situated in a continuum of possibilities going from evading the hostility to confronting it. Other forms of intermediate responses were self-segregation, verbal encounters, and seeking the intervention of a third party to cease the conflict. The emotional impact of microaggressions on Indigenous women were described as having a cumulative effect on victims that generated feelings of isolation and exclusion. This cumulative effect is remarkable because, as Hollenstein highlighted, victims find it difficult to forget the hostilities they have faced throughout their lives, which ultimately creates a collective memory to members of the Indigenous community.

Hollenstein (2008) concluded that in Ecuador schools are racialized environments where symbolic encounters are common. Schools act as mechanisms to impose ethnic boundaries where the idea of race is produced and reproduced. This study mainly analyzed microaggressions in primary and secondary schools with the intersection of women. Hollenstein's article is valuable to this study since it acknowledges the existence of microaggressions in the Ecuadorian system and establishes the need for further research in the identification and reconstruction of other racialized settings to a better understanding of this phenomenon.

\section{Ecuadorian Context and Indigenous Education}

An Ecuadorian constitution amendment in 1998 evidenced a prosperous outcome for Indigenous organizations. For the first time and after decades of constant tension among the government and Indigenous organizations, the latter were able to include demands in the Constitution. A complete chapter was dedicated to acknowledging Ecuador as a pluricultural and multi-ethnic country that celebrates diversity as an asset to 
achieve social, cultural and economic growth. This body of law guaranteed communities and indigenous nationalities to maintain, develop and enhance their identity, sense of belonging, ancestral traditions and social organizations (Constitution of Ecuador, Act 2011). Likewise, the new Constitution denied any racism or discrimination on the basis of origin and ethnic or cultural identity. In terms of supporting minorities' access to quality education, the government assured development and improvement toward intercultural bilingual instruction from elementary to higher education (Constitution of Ecuador, Act 2011).

In a further attempt to bridge prevalent gaps identified in the 1998 constitution, the Asamblea Constituyente 2008 acknowledged that pluricultural and multi-ethnic perspectives were not sufficient with respect to a more democratic and inclusive society. Indigenous movements proposed a redefinition for the Ecuadorian state. After extensive debate, pluricultural and multi-ethnic views for Ecuadorian state were removed and replaced with intercultural, plurinational, and secular ones (Asamblea Constituyente, 2008). The amendment involved granting equal value to people, accepting diversity, validating different cultures comprehensively and guaranteeing equity. In consequence, the perspective of the new Ecuadorian constitution was to walk toward a state that achieves equity, and that terminates colonial heritage (Sarango, 2016). Ideally, as pillars of the Ecuadorian state, the three principles mentioned above are mandatory and aimed at compensating marginalized groups for centuries of exclusion and oppression.

One of the mechanisms toward achieving a more democratic and inclusive society, as it is proposed in the constitution, was the provision of an education that assures all students, especially those coming from disadvantaged groups with equal 
opportunities to succeed. However, as Avalos (2012) stated, profound inequalities still mark Ecuadorian society in all realms. The most evident inequality deals with the quality of education citizens have access to because it is disproportionately distributed with enormous differences in enrollment, goal achievement, retention, and completion rates on the basis of gender, ethnicity, class, cultural background and other factors (Post, 2011).

To understand disparities, an in-depth look at the characteristics of Ecuadorian society is necessary. Oviedo and Wildemeersh (2008) described Ecuadorian society as complex in terms of ethnicity because the criteria for determining who belongs to what group are relatively ambiguous. The 2010 census determined that $8 \%$ of the Ecuadorian people — roughly one million people — identified themselves as Indigenous. However, this number might be inaccurate according to the Confederation of Indigenous Nationalities of Ecuador (Confederación de Nacionalidades Indígenas del Ecuador, CONAIE) which estimated approximately three million Indigenous people (Oviedo \& Wildemeersch, 2008). The inconsistency lies in the fact that when answering the question about ethnicity, many people prefer to identify themselves as Mestizos because this condition might give them more acceptance than being acknowledged as Indigenous (Martinez \& de la Torre, 2010).

Unfortunately, access to quality education resides not only in ethnicity but also in the distribution of wealth. Ecuador is a country with profound disparities that affect both Mestizos and Indigenous people. Distribution of services and public goods is disproportionate being Indigenous people are those who receive the fewest benefits. The National Board for Statistics and Census (Instituto Nacional de Estadística y Censos, INEC) stated that the Indigenous population continues to be the most deprived group in 
Ecuador with a poverty rate of $64.8 \%$ in 2014 (INEC, 2016). The same report highlighted poverty as responsible for depriving Indigenous people from access to education. Martinez (2002) claimed that although Indigenous people acknowledge the importance of education to escape poverty, they face challenges in providing their children with education mainly because of school expenses and the need for young workforce to plow the fields. Levels of education in Ecuador depend on the region where people live. Areas where higher proportions of Indigenous and Afro-descendant populations live are those with the lowest educational attainment level (Sánchez, 2006).

As stated before, the Ecuadorian educational system is far from being egalitarian in terms of offering all citizens the same opportunities to access education regardless of their class, gender, income, or ethnicity. Fabara (2013) revealed that a substantial number of illiterate people live in poor rural areas. The analysis demonstrated that ethnicity is a decisive marker in access to education. Illiteracy affecting Indigenous population are evident as this group showed the shortest schooling permanence compared to Mestizos with an average of 5.5 years. Statistics also showed that only $16.6 \%$ of Indigenous students finish high school. The last census reported by INEC (2010) regarding the presence of the Indigenous population in higher education revealed that Indigenous participation is scarce, especially in urban areas where Mestizos are predominant. The census showed that less than $1.2 \%$ of the students who have achieved a college degree are Indigenous. These statistics supported the fact that the Ecuadorian educational system still exhibits extreme disparities that affect deprived minorities, condemning them to an everlasting condition of poverty and oppression. As a consequence, Ecuadorian society is still obligated toward reducing the gap between the haves and the have-nots. 
Regarding how educational structures operate to systematically invalidate diversity, plurinationality, and multiculturality that results in ethnic and racial segregation, Martinez and de la Torre (2010) discussed how Indigenous students are compelled towards Mestizaje to be accepted in their institutions. According to the authors, the system does not produce homogeneous Mestizo subjects. On the contrary, they suggested that elite private institutions emphasize the value of whiteness while in middle-class schools there is an evident exclusion of students because of their race and social status. Schools for low-income students, especially those serving Indigenous communities, do not contribute to reducing gaps in terms of both racial discrimination and quality of education. To avoid bias and increase acceptance in institutions and society in general, Indigenous students switch to "Mestizaje" meaning that they abandon their traditional outfits, language, and customs in what Avalos (2012) described as the "social whitening" of people. This social whitening is essential to Indigenous students for symbolically allows them to gain power and legitimacy. However, the change leaves them in limbo because they are acknowledged neither as Mestizo because of their skin nor as indigenous because of their looks.

Martinez and de la Torre (2010) proliferated in the description of inequalities in the Ecuadorian educational system. The authors claimed that education in Ecuador does not guarantee social mobility and contributes to reproducing the status quo where the powerful reaffirm and maintain their position of dominance while the majority of the population, especially Indigenous groups, are condemned to living in poverty and exclusion. Although the constitution guarantees free access and quality of education in public institutions, equity for all citizens is still a fallacy. The authors believed that the 
national curriculum recreates colonialism given that idealized western cultures become models to follow while local customs, traditions, and people receive less credit. The system subtly pushes underrepresented students to embrace a culture they do not belong to while invalidating their ancestral principles and knowledge.

\section{The Five Faces of Oppression}

For many, society has overcome oppression since practices such as slavery in the U.S. or encomiendas, mitas y obrajes (colonial institutions that forced Indigenous people to work for extraneous hours for the Spanish in servitude, mining, and manual labor) in Ecuador were surmounted long time ago. However, the $21^{\text {st }}$ century exhibits more elaborate manifestations of oppression that condemns millions of people to live in the margins of society enduring all kinds of hardships. Oppressed people suffering is explained for no particular reason other than belonging to a group that is disadvantaged or historically vulnerable.

Young (1990), a U.S.-based political theorist, philosopher and feminist who based her work and research on issues related to theories of justice, democratic theory, feminist theory and social difference, penned The Five Faces of Oppression where she explains the prevalence of oppression in much of modern social experience and offers a framework that facilitates its diagnosis. In her work, Young calls for movements to adopt different modes of analysis that lead to recognize oppression. Since its first release in 1990, Young's multi-nuanced idea of oppression has provided the foundation to numerous studies to identify the prevalence and manifestations of oppression in different social groups. In her seminal article, Young (2004) provides a straightforward description of the oppression categories that enables researchers to operationalize each component 
separately. The model can be used to examine the reality of any society or organization to determine if a given group is being subject to unjust treatment or control. The five faces of oppression proposed by Young are violence, exploitation, marginalization, powerlessness, and cultural imperialism.

Oppression is a complex term that is usually misunderstood or underestimated mainly because people believe oppression is a matter of the past. In its simplest definition, oppression is the extended or undeserved treatment or control of a group over to other with a heavy connotation of colonialism and conquest. The colonial attribute assigned to oppression explains why people regard oppression as an event that occurred in the past with little repercussion to the present. The Oxford Dictionary of Philosophy (2008) defines oppression as the "unjustifiable placing of a burden on someone or some group, by interfering with their powers, interests, or opportunities. Oppression may be deliberate or an unintended outcome of social arrangements; it may be recognized for what it is or may go unremarked even by those oppressed" (p. 342). This more comprehensive description highlights two features of oppression. First, oppression is an indefensible practice that affects people's capacities by interfering with their development and taking away their power. Second, because oppression can take subtle manifestations, it is in most cases, difficult to identify.

Young (2004) sees oppression as a structural concept that refers to "systemic constraints on groups that are not necessarily the result of the intentions of a tyrant" (p. 41). Oppression in this sense is structural, rather than the result of a few people's choices or policies. Oppression causes are "embedded in unquestioned norms, habits, and symbols, in the assumptions underlying institutional rules and the collective 
consequences of following those rules" (p. 41). For Young, oppression extends to all components of society causing a myriad of injustices against groups because of involuntary assumptions of people. These injustices take place in everyday realms such as social interactions, media, and public spheres. Oppression is structural because it permeates rulers and the laws they enforce that oppress people in economic, political and cultural realms (Young, 2004).

Thus, oppression is an intricate concept implying the control or reduction of a social group. Young (2004) recommends that researchers first recognize and understand the existence of what she calls social groups before analyzing if a determined population is subject of oppression. According to Young (2004), a social group is not limited by surface traits such as gender, race, or any other observable characteristic. Social groups arise "in the encounter and interaction between social collectivities that experience some differences in their way of life and forms of association, even if they regard themselves as belonging to the same society" (p. 42). Additionally, a social group is acknowledged by a sense of identity that comes from the identification of a person's feelings with a specific group in accordance with the common cultural forms, social situation, and history that the members of a group share in common. Furthermore, social groups exist only in relation to other social groups and the synergy between them. Young supports the idea of society integrated by different social groups. However, Young (2009) maintains that the existence of groups in society is beneficial only when institutions promote the respect for group differences without oppression.

Under Young's definition and conception of group, Indigenous people meet all the conditions to be acknowledged as a social group. In their day-to-day activities and 
from different scenarios, Indigenous people establish constant interactions with other social groups that purportedly should produce benefit for both parties. Likewise, Indigenous people identify themselves with a common history and culture that shape their identity. Indigenous people also share particular ways of living, knowing and understanding the world that differ with conceptions of Mestizo or White people. Indigenous people's underlying representations, ethnicity, cultural products, practices and perspectives represent their particular view of the world that produces a sense of unity among members. From these viewpoints, Indigenous people are a social group influencing one another and the social landscape around them.

The analysis to determine if Indigenous people as a social group are target of oppression comes from a careful assessment of the five faces of oppression offered by Young (2004). Her framework depends on the examination of the presence of one or more of the five conditions the author describes as exploitation, marginalization, powerlessness, cultural imperialism, and violence. The occurrence of any of these conditions constitutes enough evidence of the oppression of a social group:

- Exploitation. For Young, exploitation goes beyond the Marxist conception of means of production, private ownership and labor value that produce the transfer of power from one group to another. Exploitation is not limited to inequality of status. Exploitation also involves the weakening and even removal of people's control of their lives and freedom to make their own decisions. In addition, exploited people, suffer from deprivation of essential components of a person's wellbeing such as self-respect Young (2004). Indigenous people in Ecuador are exploited. Sánchez (2006) stated that even if Indigenous people have access to 
education, they obtain the lowest economic returns because of a discriminatory labor market. The wage indigenous people receive is lower compared to the wage a Mestizo or White person would receive for the same type of work provoking inequality of status. Another example to illustrate exploitation is that most of the Indigenous people's arduous work in the fields does not return to them in the proportion to their effort and time investment. Indigenous people's labor benefits Mestizos and Whites because although Indigenous people take the best of their crops to the market, the payment they receive is not enough to satisfy needs such as good education and health care. Poverty in Indigenous populations in Ecuador reached $64.8 \%$ in 2014 (INEC, 2016).

Although for decades Indigenous people have struggled for equity, their efforts have encountered enormous resistance because Ecuadorian society is determined by a solid structure of exploitation against this population. As Ibarra (1992) stated, it is unlikely that groups maintaining long-standing power, want to give away their privileges. However, the transfer of energies from Indigenous people to other social groups is not limited to labor. Since Indigenous people receive the least investment from the government in education, health services, electricity, Internet, drinking water, transportation, and other types of public services; it is dominant groups receiving those benefits at the expense of Indigenous people. Young (2004) asserts that race is the main reason behind exploitation because people are classified in two main groups-one as superior because of their skin color, and the others being the superior those who have more privileges in society. In this sense, Indigenous people are usually assigned to 
low-wage jobs especially in agriculture, and domestic service while high-income jobs are reserved to Mestizo or White entailing a transfer of energies from the servers to the served who enhance their status (Young, 1990).

- Marginalization. Marginalization entails treating a person, a group or an idea as being irrelevant or marginal. People are marginalized when the system of labor cannot or will not use their workforce for considering them as underclass. Because people are underclass, they are condemned to permanently reside in social marginality. This marginalization happens mainly because of racial reasons. However, a myriad of other factors also results in excluding individuals such as age, instruction, marital status, and mental or physical disability (Young, 2004). The Five Faces of Oppression acknowledges marginalization as the most dangerous form of coercion because it sentences people to deprivation and in extreme cases extermination. In order to mitigate the starvation of social groups, societies have devised mechanisms like welfare payments and special services. However, this apparent benevolence hides a second intention. Marginalized people receiving government assistance become dependent and therefore target the often dictatorial and intrusive control of social service that demands conditions people must satisfy in an effort to maintain their assistance. In complying with welfare requirements, marginalized people lose their freedom, power, autonomy, and privacy. This dependency is not oppressive by itself but generates feelings of uselessness, boredom, and lack of self-respect for people, depriving them from exercising their capacities in a context of recognition and interaction (Young, 2004). 
In Ecuador, the government provides vulnerable groups like the elderly, single parents with young children, people with terminal diseases, and people with disabilities with a monthly stipend that ranges from $\$ 50$ to $\$ 150$ that helps them satisfy basic needs. The amount depends on a number of factors, for example, the number of children in the family, type of disability, and age. Families receiving this incentive must prove they are living in extreme poverty and that they do not have any income other than the government's stipend. Although some advantages for people in extreme poverty have been reported after receiving the human development bonus in Ecuador, poverty rates have not decreased significantly in the last decade.

- Powerlessness. Young (2004) distinguishes between professionals and nonprofessionals in the view that the former group receives more privilege compared to the latter. This distinction generates a division of labor and status where nonprofessionals are oppressed and therefore powerless. Professionals usually reach directive positions in society from which they dictate policies, have authority and make decisions that may affect nonprofessionals. Therefore, the powerless (nonprofessionals) lack the authority, status, and sense of self that professionals tend to have. The status of privilege professionals reach because of their capacities produces their progressive development, autonomy, and respectability. Nonprofessionals lack these three conditions in their lives, which produce oppression for this social group (Young, 2004, p. 55). Progressive development comes from college education and acquisition of specialized knowledge that entails professionals working with abstract symbols and concepts. 
Progressive development gives professionals the autonomy and authority they exercise over nonprofessionals. Being able to make decisions and having authority gives professionals respectability. In their status as professionals, this social group benefits from respect, freedom of expression, and greater value compared to nonprofessionals. In consequence, nonprofessionals exhibit less power than those who have had the opportunity to achieve a university degree. The division of labor between people who perform at the conceptual level and those at the manual level also produces division in myriad other aspects such as access to prestigious schools, neighborhoods, towns, food, clothes, music, vacation, health, insurance and the like. As previously reported, INEC (2010) reported that in Ecuador less than $1.2 \%$ of the students who have achieved a college degree are Indigenous; thus, we may understand that there is scarcity of Indigenous professionals in every field. Consequently, Indigenous people as a social group have been systematically banned from achieving progressive development, autonomy, respectability and therefore power in comparison to Mestizos and White citizens.

- Cultural Imperialism. Exploitation, marginalization, and powerlessness are the consequence of social division of labor that determines the position of social groups (Young, 2004). These faces of oppression are structural and institutional and define people's material existence. However, there are other types of oppression that do not depend on institutional structures. These forms of oppression are more difficult to determine because a careful scrutiny into society arrangement is required. Cultural imperialism refers to "[experiencing] how the 
dominant meanings of a society render the particular perspective of one's group invisible at the same time as they stereotype one's group and mark it out as the Other" (Young, 2004, p. 56). Cultural imperialism implies the imposition of the interpretation of the world of one dominant group on other that has to assimilate the perspectives of the dominant passively. In this way, one group remains as prevailing while the other occupies a position as inferior. The culture of the dominant group becomes the social paragon that has to be accepted by other groups, which originates differences between groups. The paradox according to Young (2004) is that dominated groups are "both marked by stereotypes and at the same time rendered invisible" (p. 57). Stereotypes are so engrained in society that they are not noticed in society nor contested by people. At the same time, dominated people make efforts to both assimilate in the dominant culture and resemble it to gain more prestige, validation, legitimacy and acceptance. Culturally dominated groups live their lives in a state of double consciousness. On one hand, the dominated people refuse to accept their devalued, objectified, stereotyped visions of themselves; on the other, however, their desire for recognition as humans provokes judgment from the powerful who only see differences and inferiority (Young, 2004). Injustice of cultural imperialism lies in that oppressed groups' experiences and viewpoints of social life do not touch the dominant groups while the dominant groups impose theirs to the oppressed.

Cultural imperialism is cultural in that a different set of habits, behaviors, language, and social norms are introduced to oppressed groups causing a voluntary or involuntary reshaping of their ways of living, beliefs or values. It is 
imperialism inasmuch as the imposing community gets authority over the oppressed population by changing or swapping features of the subordinate culture (Weynand, 2016).

Cultural imperialism in Ecuadorian Indigenous populations started with the Spanish conquest and has prevailed for more than five centuries. Throughout history, cultural imperialism has taken different nuances. Today, Mestizos and Blancos $^{4}$ impose cultural imperialism. They enforce Whiteness as a parameter of being civilized, accepted, and normal while Indigenism is portrayed as secondclass, inferior, or secondary. Ecuadorian society opted for Mestizaje as an option for the elites to homogenize society under a unique cultural code that defines and shapes Ecuadorian society because being Mestizo is acceptable while being Indigenous is not (Santillana, 2013).

- Violence. The concept of violence has been reassessed throughout the years. Many forms of violence are no longer overt but covert. Kilby (2013) argues that today, violence is gentle and often invisible making it difficult to grasp. Violence against oppressed groups is not as observable as it was years ago. However, it has become more intricate and elusive. Because violence is covert, it is to some extent tolerated and accepted by members of society (Sigler, 1995). Oppression generates violence in that people live in fear of attacks, humiliations or abuse simply because they do not belong to a dominant group. Millions of people live their lives frightened of unreasonable and unprovoked attacks with the simple

\footnotetext{
${ }^{4}$ Blancos. In Ecuador, some people self-identify as Blancos despite they know that there is a fusion of Indigenous genes in their heritage. Also referred as White-Mestizos.
} 
purpose degrading, stigmatizing, and humiliating them. In her work, Young (2004) considers both physical and nonphysical violence in the form of harassment, intimidation, or ridicule as manifestations of violence that threaten people's lives. She argues that "what makes violence a phenomenon of social injustice, and not merely an individual moral wrong, is its systemic character, its existence as social practice" (p. 59). Violence against social groups, especially those considered as vulnerable, is according to Young institutionalized and systemic because society promotes, sustains, or facilitates the perpetration of violent acts against members of specific groups.

Racism entails violence. As a social group, Ecuadorian Indigenous people suffer systemic and institutionalized violence because racism is deeply rooted in Ecuadorian society. As Almeida (1996) affirms, racism flows smoothly in every process of socialization generating conflict in micro-spaces where incongruences are evident.

As a framework for analyzing oppression, Young's work offers a view of this phenomenon as the intersection of different aspects rather than as a unity. The advantages of looking at oppression as the convergence of multiple facets lies in that this way of conceiving oppression avoids the exclusion of social groups and reductions of criteria. Young' criteria to determine oppression are easy to identify even for those who doubt its existence. The framework does not place hierarchy between each aspect but facilitates identifying and comparing the different manifestations of oppression. By providing an account of the prevailing symptoms of oppression Indigenous students endure, we may be able to overcome stereotypical assumptions that society refuses to acknowledge. 
The purpose of this literature review was to present an overview of previous literature on microaggressions, its connection to self-esteem, self-efficacy, and responses and mechanisms people use to cope with racial hostilities. The review also looked at Indigenous populations, schooling, and manifestations of microaggressions in Ecuadorian context. Finally, we included an analysis of the five faces of oppression as the framework that will assist this study to identify prevalence of oppression of Indigenous students because of being targets of microaggressions.

The research reviewed on microaggressions reveals that racial hostilities against underrepresented groups are still rampant in today's educational settings. Furthermore, it is evident that despite the number of studies on microaggressions, a deeper understanding of how this phenomenon behaves and affects college students in different contexts is necessary for the benefit of targets, higher education institutions, and society at large. Microaggressions deserve attention, especially in settings where racial issues are in interdiction because of colorblindness. This field of inquiry is also relevant to creating awareness not only in targets but also in the community toward making campus environments spaces where everybody feels safe and appreciated.

In regard to Ecuador, the analysis of the literature suggests a need to determine the most common type of microaggressions Indigenous students are exposed to, as well as the understanding of how these hostilities relate to their self-esteem and self-efficacy. Likewise, there is demand for knowing how victims of microaggressions respond to and cope with microaggressive events. A key goal of this study is discovering as many of these aspects as possible. 


\section{CHAPTER THREE}

\section{METHODS}

Chapter Three describes a mixed methods study aimed at examining types of microaggressions affecting Indigenous undergraduate students while pursuing a Bachelors' degree in an Ecuadorian institution as well as the associations of such racial hostilities on their self-efficacy and self-esteem. It sought to explain the experience of Indigenous students in terms of how they respond and react to racial hostilities and how they use mechanisms to cope with microaggressions. With these ideas in mind, I put forward the following research questions:

1. What are the most/least common types of racial microaggressions that Indigenous students experience while pursuing a degree in a public higher education institution?

2. What is the association between microaggressions and Indigenous students' self-esteem?

3. What is the association between microaggressions and Indigenous students' self-efficacy?

4. How do Indigenous students experience racial microaggressions while pursuing a degree in a public higher education institution on issues of (a) responses to microaggressions, and (b) mechanisms they use to cope with microaggressions?

The questions posed in this study required the use of mixed methodologies. Mixed methods research merges quantitative (close-ended) and qualitative (open-ended) research procedures to integrate information so that one procedure builds on or extends 
the other. Each method contributes to a deeper understanding of the phenomenon.

Johnson et al. (2007) identified motives for researchers to use mixed methods research:

- Triangulating to enhance the validity of the study. The collection of data through more than one method makes it possible to corroborate findings.

- Seeking complementarity to enrich, clarify or elucidate results from one method with results from another method.

- Developing more information from one method to inform the other approach.

- Discovering paradoxes and contradictions. These inconsistencies may lead researchers to initiate or reframe the study research question.

- Expanding the breadth and range of inquiry and get in-depth understanding of the phenomenon (pp. 115-116).

Aside from the practical reasons mixed method approach entails, a fundamental motivation for adopting this stance for this research was that mixed methods help researchers look at issues containing social justice and human condition from both objective and subjective perspectives. Given the complexity of microaggressions, the combination of approaches was crucial for the purposes of this study. As a researcher, I was interested in microaggressions that are a form of oppressing underrepresented groups in society generating social conflict and inequities. Given the relevance of the topic, it needed a broad analysis that contained rigorous and persuasive methods in the search for advocacy and justice.

The following section includes a comparison between positivist and naturalistic approaches to research as an introduction to mixed methods approach and the rationale to 
the use of the sequential transformative model. Next, both quantitative and qualitative studies are described in detail.

\section{Comparing Positivist and Naturalistic Approaches}

Positivism appeared as a theoretical paradigm in the $19^{\text {th }}$ century. Positivist approaches to research were considered as the only valid method for creating new knowledge. Science was a synonym of lab work where experiments were performed to test the hypothesis. The main characteristic of positivist-based research is the posing of hypotheses tested by deductive methods (Neurath, 1973). Hypotheses testing follows a systematic procedure to retain or reject assumptions (Morse \& Field, 1995). Different procedures are followed to achieve validity and reliability criteria. Objectivity is central to researchers who can only rely on numerical data and whose beliefs, passions, politics, and thought cannot intervene in the inquiry process. Another characteristic of positivist approaches is that the application of sophisticated statistical models determines relationships between phenomena. For positivist approaches, the reality is stable, and variables are constant (Neurath, 1973).

Despite the wide acceptance of quantitative methods in the scientific community, they do present limitations. Quantitative research does not always explain the full intricacy of human experience or perceptions. Quantitative methods are useful to describe what but not necessarily how a phenomenon operates because it focuses only on those aspects of social behavior that can be quantified (Rahman, 2017).

On the other side of the spectrum, though, is naturalistic inquiry. The naturalistic paradigm relies on the belief that reality is not immutable. Reality is composed of multiple interdependent factors that cannot be explained by mathematical formulas 
(Guba, 1990). Guba proposed the idea of different realities that occur as multifaceted mental constructions that are specific to the individuals who hold them. The author stated that naturalistic methods contribute to understanding human experience from the perspective of specificity and uniqueness. Guba also suggested that another characteristic of the naturalistic approach is that it concedes importance to the researchers' values and beliefs. Researchers then are not mere instruments for applying formulas, researchers bring to the inquiry process their experience. The nature of qualitative research is holistic and inductive. It neither formulates hypotheses nor controls the conditions of the study because its aim to gain deep understanding of a phenomenon.

In contrast to positivistic stands, naturalistic approaches consider phenomena as integral occurrences rather than as fragmented pieces in a laboratory. For researchers adopting naturalistic approaches, data analysis is inductive, and instrumentation is subjective (Tavakol \& Zeinaloo, 2004). Maxwell (2013) advocated for qualitative research because it connects knowledge with the conjunction of several aspects such as universe of meanings, motives, aspirations, beliefs, values, and attitudes that establish understanding of relationships, processes, and phenomena that cannot be reduced to the operationalization of variables.

However, qualitative approaches to research pose some limitations. The major limitation is that small sample sizes used in qualitative studies hinder generalizability to the population. Another limitation is that conclusions need to be carefully interpreted since they belong to a specific community with particular attributes. Despite limitations, qualitative approach offers a detailed practice for researchers to describe aspects of human behavior that are not possible to quantify. 


\section{The Mixed Methods Research Paradigm}

A third dominant research paradigm to research is mixed methods. Creswell (2008) emphasized the importance of using the strengths of qualitative and quantitative analysis in a mixed method approach to inquiry. Creswell (2008) also stressed the importance of combining both approaches to gain more insights and expand the understanding of research problems. Mixed research methodology has its origin on the endless debates to view the world as a universal truth or as multiple relative truths. Mixed methods situate in the middle of the spectrum.

Mixed methods research first emerged in the late 1980s. A mixed methods approach is then new in the field of research and still in the process of construction.

Although there are still some discrepancies with definitions and philosophical foundations, what is clear for theorists is that mixed methods is an approach of centrality (Creamer, 2018). The most accepted definition for mixed methods research is provided by Creswell and Plano-Clark (2011) as

Mixed method is a research design with philosophical assumptions as well as methods of inquiry. As a methodology, it involves philosophical assumptions that guide the direction of the collection and analysis and the mixing of qualitative and quantitative approaches in many phases of the research project. As a method, it focuses on collecting, analyzing, and mixing both quantitative and qualitative data in a single study or series of studies. Its central premise is that the use of quantitative and qualitative approaches, in combination, provides a better understanding of research problems than either method alone. (p. 5)

Creamer (2018) analyzed this definition focusing on the conception of mixed approaches as both method and methodology. As methodology, mixed methods underpins four philosophical assumptions. First, mixed methodology does not deem qualitative and quantitative data and methods as incompatible but complementary, 
meaning that the combination of both enhances the qualities of each other. In the same vein, the combination of both approaches makes findings more robust and dependable. Next, Creamer emphasized the idea of added value that translates into the improvement of the characteristics of both quantitative and qualitative approaches. A third assumption referred to increasing validity since multiple types of data often better inform conclusions. Finally, Creamer discussed the complementarity argument in the sense that the mixed approach compensates the weaknesses of both qualitative and quantitative methodologies. As a method, the combination of quantitative and qualitative parts lead to a unique product that has been enriched by the contributions from both primary constituents.

Creswell (2008) recommended that researchers using mixed methods consider some aspects that influence the design of procedures. Researchers need to reflect on the timing for data gathering that can be sequential or concurrent. Sequential data collection occurs when data are collected at different times. The order to which data are collected, depends on the researchers' interest. The concurrent method implies collecting both quantitative and qualitative data simultaneously which, according to Creswell, is in many cases unworkable. By the same token, researchers need to decide about the weight or priority they will give to data. Any priority will depend on researchers' interest, the audience, or the emphasis researchers want to give to the information collected with either method. Creswell also asserts that when and how to mix data are questions researchers need to define. The moment at which data will be combined can take place at three points: data collection, data analysis, and interpretation. How the information is mixed in the study takes three forms: quantitative and qualitative data can be combined 
on one end of the process, they can keep divided at the end of the continuum, or they can be connected in some way between these two extremes.

A final but not unimportant consideration that researchers have to attend to is the type of mixed methods design to use in their study. Creswell (2008) identified six different mixed methods strategies: sequential transformative, sequential explanatory, sequential exploratory, concurrent triangulation, concurrent nested and concurrent transformative.

First, the sequential transformative strategy is, as Creswell (2008) stated, a twophase project supported by a theoretical lens that outlines the direction of the study. The sequential transformative approach aims to assist the lens taken by the researcher. The lens adopted overlays the whole research process and marks the path that the research has to follow to examine the problem. This means that the lens-whether it be a conceptual framework, a specific ideology, or advocacy—is paramount in guiding the study rather than the use of a specific method by itself. Sequential transformative strategies are useful for researchers interested in exploring topics related to inequality, discrimination, or injustice oriented to create sensitivity and a call for action. Aligned to the nature of sequential transformative approach, the analysis of racial microaggressions of Indigenous students in Ecuadorian higher education brings together evidence from this underrepresented group both in quantitative and qualitative form to give this social group voice, generate awareness and provoke change.

Methodologically, data collection for the quantitative or qualitative phase is indistinctive for sequential transformative approach (Creswell, 2008). Although in theory no quantitative or qualitative data receive more weight, one of them usually receives 
more importance than the other. Furthermore, the use of separate phases to data collection makes it easier for researchers to implement, describe, and share results. Although this method is the most popular among researchers, it also has some drawbacks. One limitation is that it requires researchers to have enough expertise to deal with two separate bodies of data, which to some extent makes it difficult to compare results. The characteristics of the sequential transformative approach fit the needs of this study. A more detailed rationale for adopting this strategy will be provided later in this chapter.

Second, in a sequential explanatory mixed methods approach, researchers collect quantitative data first and use this information as the foundation for a second qualitative phase. Although both sets of data are separated, a common thread connects them. In this approach, quantitative information receives more weight. According to Creswell (2008), a theory is not a sine qua non for a study adopting a sequential explanatory approach. These studies are useful when researchers believe they might obtain unexpected results from quantitative research. An apparent weakness of this method is that it is timeconsuming as data collection stages are separate.

Third, the sequential exploratory strategy collects qualitative data before quantitative. Qualitative data inform quantitative data. However, more weight is given to qualitative information. Similar to the sequential explanatory method, data are shared and mixed by a common thread. Although a theoretical foundation is essential, this component is not mandatory for sequential exploratory studies. Additionally, sequential exploratory studies are also recommended when researchers are interested in developing instruments. This design is easy to implement and report. Researchers wanting to explore a phenomenon and testing elements of an emergent theory might find in sequential 
exploratory design a good option. However, researchers using this approach should be cautious of time constraints since this design is time consuming. Another consideration for researchers is the difficulty they may face to decide which finding from the qualitative data is going to inform the quantitative component of the study (Creswell, 2008).

Fourth, concurrent triangulation comprises the use of two or more methods to confirm, cross-validate, or corroborate findings in the study. This method implies concurrent data collection to overcome the weaknesses of one of the methods in the study with the strengths of another. Ideally, both quantitative and qualitative approaches receive equal weight. However, Creswell (2008) warns researchers that the perfect balance between both approaches is difficult to achieve.

Fifth, the concurrent embedded/nested method gives quantitative or qualitative data the priority that provides support to the other. The term nested is given to the data that depends on the other. The nested data can answer a different research question that the primary method cannot. Creswell (2008) suggested that data should reside side by side as two different pictures of the phenomenon that gives an optimal view of the problem. In concurrent embedded studies, a theoretical perspective that guides the process is recommended. The purpose of researchers using this method is to describe an aspect of the problem that has not been addressed by the limitations of either qualitative or quantitative procedures. A disadvantage is that some discrepancies may appear between the two sets of data Creswell (2008).

Finally, concurrent transformative studies use a specific theoretical perspective and collect data concurrently. This method is guided by the purpose of the study or the research questions. Creswell (2008) asserted that this model could take on both 
triangulation or embedded approaches. Regarding mixing data, the researcher can merge, connect, or integrate them. The transformative framework guiding this type of study is an advantage for researchers using this model.

\section{Rationale for Sequential Transformative Approach}

Microaggressions is a complex and sensitive topic. Restricting the analysis to identifying the most prevalent types of microaggressions Indigenous students encounter and determining the extent to which these microaggressions correlate to self-efficacy and self-esteem is not enough to understand this issue holistically. There is also need to complement findings with examples or comments from the lived experiences of Indigenous students. Therefore, the reason to expand information about how Indigenous students react to and cope with microaggressions was required to a more in-depth view of racial hostilities in Ecuadorian higher education institutions. Mixed methods research in the form of sequential transformative approach offered the flexibility for the researcher to look at microaggressions as a social problem in a more comprehensive way.

This study analyzed microaggressions affecting Indigenous students in Ecuadorian higher education. Since the topic is socially sensitive, the sequential transformative approach as a new methodology concerned to social inquiry, offered the possibility to, as Sweetman, Badiee, and Creswell (2010) suggest, embrace ideals of social justice to shape a more equitable society. Similarly, the sequential transformative approach fills the need for more advocacy research within the field of mixed methods (Creswell \& Plano Clark, 2007). Sweetman et al. (2010) also highlight the applicability of tranformative approaches to analyzing issues of oppresion and domination in favor of disadvantaged groups who are targets of inequities because of their race, ethnicity, 
disability, or sexual orientation. The way this study was conceived, attended to Sweetman' et al. recommendations for researchers interested in using transformative research approaches. In this study, the transformative research approach assisted the lens taken by the researcher, helped to explore topics of inequality, diversity and oppression, and facilitated the sharing of results

The sequential transformative method was the most suitable for this study because it requires the use of a lens that, in this case, is Young's (2004) Five Faces of Oppression. As mentioned in previous chapters, Young's framework is useful to determine the prevalence of oppressive tendencies that much of modern society still practices against disadvantaged groups and facilitates its diagnosis. Young's framework aligns with the purpose and research questions in this study since it calls for social justice and advocacy for the benefit of systematically relegated people. The lens I am adopting to this study, as Creswell (2008) suggested, "creates sensitivity to collecting data from marginalized or underrepresented groups and ends with a call for action" (p. 212). Creswell also emphasizes that the use of both quantitative and qualitative data sets gives "voice to diverse perspectives to better advocate for participants" (p. 212). Despite the benefits of using transformative approaches to research, Creswell (2008) warns researchers of some limitations. One limitation is that there are not many studies informing the use of this method. This scarcity resulted in insufficient direction for the researcher with respect to how to use the transformative approach properly.

\section{Quantitative Component}

The quantitative component of this study answered the first three questions related to the most/least common types of microaggressions Indigenous students 
encounter in their everyday activities, the associations between microaggressions and students' self-esteem, and the associations between microaggressions and students' selfefficacy. To answer these questions, data from three surveys were collected: The Racial and Ethnic Microaggressions Scale Nadal (2011), the Spanish version of Rosenberg's Self-Esteem Scale offered by Albo et al. (2007), and the Spanish translation of Schwarzer and Jerusalem's General Self-Efficacy Scale given by Brenlla et al. (2010).

\section{Study Participants and Inclusion Criteria}

Participants for the study were recruited via advertisements posted on flannel boards and public places all over the campus where the study was carried out (Appendix B). An oral recruitment announcement was also devised for teachers working at the institution who willingly spread the word to their students (Appendix C). Ary et al. (2002) highlighted the advantages of public advertisement and word-of-mouth strategies especially when recruiting specific populations as in the present study. The authors state that these methods are affordable and offer effective results. An additional recruitment approach to call for participants was snowballing. Participants were encouraged to spread the word about the study to their peers. Snowball is useful because previously selected subjects suggest other participants who fit the requirements of the sample (Ari et al., 2002). Students interested in taking the survey filled a sign-up sheet with their contact information. The researcher contacted the participants and established a schedule for the students to take the surveys. The participants of the study were 127 Indigenous students who voluntarily agreed to participate in the study. However, the final sample consisted of 112 participants. Respondents were deleted for three reasons: having missed the survey 
meeting, not satisfying eligibility criteria requirements, and missing the background information component of the questionnaire.

As an essential requirement, participants had to be currently enrolled in any major at the institution where the study was conducted. Additionally, three criteria for participation were decisive in this study: The most important criterion for selection was that participants self-identified as Indigenous. All participants in this study satisfied this criterion. Another condition was that students had to be at least in their sophomore year. This requirement was fundamental to the present study because, after their first year, sophomores have already acquired some experience in navigating university and they are usually more aware of their environment. The last condition was their free will in deciding whether to participate or not. The researcher avoided any kind of coercion exercised by teachers, administrative or authorities. As a researcher, I believe that by accepting to participate voluntarily, participants demonstrated their interest in the topic that ultimately resulted in providing more genuine and truthful answers.

\section{Quantitative Data Collection Procedures}

Three paper-based surveys were administered to participants: the REMS, the Rosenberg Self-Esteem Scale, and the General Self-Efficacy Scale. In response to institutional policies in the institution where the data were collected, an approval procedure was necessary before distributing the instruments to the target population. The Vice-president of Academic Affairs gave authorization to conduct the study (Please see Appendix D). The Office of Academic Affairs copied the authorization letter to the deans of each college who respectively approved data collection. In some cases, and attending to teachers' suggestions, classroom visits were necessary for data collection. The protocol 
for each class visit consisted of handing teachers the approved documents and providing them with a brief explanation of the aims of the study.

The three instruments were administered simultaneously. For convenience, the researcher was present while students took the surveys to assure participation of the target population and hopefully increase the response rate (Mathers et al., 2007). Another advantage of delivering the surveys in the classroom is that if students are uncertain about the content of a question, they can ask for clarification from the researcher. Reja et al. (2003) suggested that the presence of the researcher is desirable, especially when participants are not motivated enough to complete the questionnaire or when respondents have difficulties understanding and following instructions. Finally, another strategy for increasing the response rate for the study was to create an appropriate survey environment. In every visit, the researcher gave a brief overview of the purpose of the survey, as well as the goals regarding its application.

Since microaggressions is a sensitive topic, the brief introduction also included an explanation of aspects related to the confidentiality and anonymity of the information. Explaining aspects related to confidentiality and anonymity was necessary since students would feel more confident in answering the survey increasing their cooperation in the process. Likewise, I offered to help respondents in the case they did not understand how to proceed with the questionnaire.

The rationale for having adopted a paper-based survey instead of an online survey also attended to specific characteristics and needs of the population in this study. First, Indigenous students coming from communities likely have limited access to computers inside and outside the campus. Although a few of them may have a personal computer at 
home, there might not be internet access in their towns, a key factor which could hinder response rate. Another reason was that although the institution provided students with an email address when they first registered in the university, students did not use it regularly. Therefore, contacting them through regular email encompassed limitations.

Finally, the self-administered questionnaire mode for the administration of the three instruments was suitable for the study because the topic has not been explored before in the Ecuadorian context and is sensitive. In this sense, Bishop and Fisher (1995) recommended that, when studying delicate issues, self-administered questionnaires give participants privacy, thereby fostering the free expression of their opinions with more confidence compared to other procedures. Furthermore, self-administered questionnary as a mode of data collection is recommended when dealing with populations that may be marginalized — which indeed is a factor in this case.

\section{Quantitative Data Analysis}

After collecting information from the three instruments, the first step was to determine the occurrence of the five types of microaggressions from the REMS. To achieve this goal, descriptive statistics was used to identify the primary characteristics of data, organize, and summarize information (Ari et al., 2002; Johnson \& Christensen, 2000). The descriptive analysis performed an accurate description of microaggressions of Indigenous students and helped identify the most and the least common types of hostilities they experience. In a second phase, correlation analysis was performed to determine the statistical relationship between the types of microaggressions with participants' self-esteem and self-efficacy respectively. The use of correlation in the study is justified because of the interest in establishing the degree of association between 
the variables. There was no manipulation of the independent variable (microaggressions) because this element is assumed to be a constant in the environment where students develop their academic activities; therefore, it was impractical and even unethical to manipulate it.

\section{Sampling}

Indigenous students enrolled in a public higher education institution located in Riobamba, one of the most highly populated Indigenous cities in central Ecuador, took the three self-administered surveys. The reason for having chosen Riobamba as the location for my study is that the city has a notable history of tense relations between two racial groups identified as Whites/Mestizos on the one hand and the Quichua/Indigenous population on the other (Klaufus, 2012). Klaufus stated that because Riobamba has become a multiethnic city, racialized incidents are ordinary yet concealed. In Riobamba, the presence of Indigenous people is to some degree considered as arbitrary and troublesome for the city (Klaufus, 2012). Despite tensions, Indigenous people have managed to access higher education albeit in a reduced number compared to Mestizos. The 32\% Indigenous population and the specific characteristics of Riobamba's environment justify having designated this context for the present study.

For my study, Indigenous students attending a Mestizo majority university took three questionnaires: the REMS, the Rosenberg Self-Esteem Scale, and the General SelfEfficacy Scale. The REMS identified the most and least prevalent types of racial macroaggressions students face in their daily activities: assumptions of inferiority, second-class citizenry and criminality, microinvalidations, exoticization, assumptions of similarity, environmental microaggressions and workplace and school microaggressions. 
The Rosenberg Self-Esteem Scale examined self-esteem by asking participants to reflect on their feelings. The general self-efficacy instrument assessed students' self-efficacy to measure their belief in their ability to react and cope with challenging situations.

The office of the register reported that 686 students attending this public university identified themselves as Indigenous in the university admissions survey; this corresponds to $7 \%$ of the college population. The information summarized in Table 1 presents the distribution of Indigenous students by college. The information was provided by the office of the registrar and corresponded to the Fall 2019 semester.

\section{Table 1}

Students Self-identified as Indigenous by College Updated to Fall 2019

\begin{tabular}{lcc}
\hline College & Ethnicity & Total of Students \\
\hline College of Education & Indigenous & 170 \\
College of Health Sciences & Indigenous & 129 \\
College of Political Sciences and & Indigenous & 196 \\
Administration & & \\
College of Engineering & Indigenous & 191 \\
Total Students & & 686 \\
\hline
\end{tabular}

Source: UNACH Department of Development and Technology Systems

The quantitative portion of the study used non-probability sampling that does not involve random selection of subjects (Etikan et al., 2016). Despite restrictions resulting from the subjective nature in selecting the sample, the author recommended nonprobability sampling in situations where randomization is not suitable because of implementation, time, workforce, and resources constraints. Likewise, Etikan et al. (2016) suggested that for social science researchers, using non-probability sampling is suitable because there may be circumstances where it is not practical, feasible, or theoretically sensible to do random sampling. 
The non-probability approach in the form of convenience sampling technique was selected for the quantitative component of the study since "the target population meets certain practical criteria, such as easy accessibility, geographical proximity, availability at a given time, or the willingness to participate" (Etikan et al., 2016, p. 1). Additionally, I work for the institution where the study was conducted thereby permitting me easy access to students. Convenience sampling was affordable, easy, and practical because the prospective participants were readily available. Etikan et al. (2016) indicated, "the main assumption associated with convenience sampling is that the members of the target population are homogenous" (p. 2). The author's assertion implied that results obtained from the population selected by convenience method would not differ from results obtained by using a random sampling procedure.

Participants were recruited by posting print advertisements on flannel boards located across the campus. Another method was through snowball sampling where participants were encouraged to spread the word about the survey to their peers who met the eligibility criteria. We expected to have from 15 to $20 \%$ of Indigenous students to take the instruments - that is approximately 120 students. The final number of students for the study was 112 .

\section{Research Question One. What are the Most/Least Common Types of Racial Microaggressions that Indigenous Students Experience while Pursuing a Degree in a Public Higher Education Institution?}

In reviewing the literature, Nadal's (2011) Racial and Ethnic Microaggressions Scale (REMS) has been widely used in several studies (Helm, 2013; McCullah, 2007; Nadal et al., 2017; Thai et al., 2017). The instrument consists of a six-factor model 
analyzing the following: assumptions of inferiority, second-class citizenry and assumptions of criminality, microinvalidations, exoticization/assumptions of similarity, environmental microaggressions and workplace and school microaggressions. In his study, Nadal surveyed college students belonging to Latin, Asian Americans, and multiracial backgrounds. Nadal followed procedures to create the final version of the survey achieving evidence of satisfactory internal consistency and validity through correlations with other scales used for individuals of all racial minority groups.

Nadal's REMS survey for microaggressions has reported a Cronbach's level of overall internal consistency $\alpha=.912$ and subscales ranging from .783 to .873 , indicating that the instrument quite reliable. Statements for each of the six factors were revised in two phases for clarity and use the first person in the singular to state opinions or feelings in a more direct manner.

Nadal concluded that the survey was useful to measure racial microaggressions in the six components. The author recommended the use of the survey to assist researchers in investigating various aspects related to microaggressions. However, he also suggested that researchers conducting similar studies could add or alter items to the survey according to their specific needs (Nadal, 2011).

The survey consists of a 45-question survey using a 5-point scale. Participants taking the survey indicate their perception of the number of times a microaggression had occurred to them in the past six months on a scale from one to five as represented in Table 2. 
The number of questions for each component in the survey varies from five to nine. The survey consists of a set of randomized items displayed into one uncategorized list. Table 3 shows the number of items for each component.

\section{Table 2}

Racial and Ethnic Microaggressions Scale Frequency of Event

\begin{tabular}{cl}
\hline Assigned value & Times a microaggression occurred in the past six months \\
\hline 1 & I did not experience this event in the past six months \\
2 & I experienced this event $1-3$ times in the past six months \\
3 & I experienced this event $4-6$ times in the past six months \\
4 & I experienced this event $7-9$ times in the past six months \\
5 & I experienced this event 10 or more times in the past six months \\
\hline
\end{tabular}

\section{Table 3}

Racial and Ethnic Microaggressions Scale Items by Component

\begin{tabular}{llc}
\hline & Component & $\begin{array}{c}\text { Number of } \\
\text { items }\end{array}$ \\
\hline 1 & Assumptions of Inferiority & 8 \\
2 & Second-Class Citizenry and Assumptions of Criminality & 7 \\
3 & Microinvalidations & 9 \\
4 & Exoticization/Assumptions of Similarity & 9 \\
5 & Environmental Microaggressions & 7 \\
6 & Workplace and School Microaggressions; & 5 \\
& Total Items & 45 \\
\hline
\end{tabular}

\section{Definition and Examples of Constructs in the REMS Survey}

The survey considers microaggressions defined as "brief and commonplace daily verbal, nonverbal, behavioral, or environmental slights, snubs, or insults, whether intentional or unintentional, that communicate hostile, derogatory, or negative messages to target persons based solely upon their marginalized group membership" (Sue et al., 2007). Targets of microaggressive acts are not just spotted by race but also by any other 
characteristic such as ethnicity, nationality, religion, sexual orientation, gender, or disability. The current study examined racial microaggressions in the six categories identified in the Racial and Ethnic Microaggressions Scale Nadal (2011): assumptions of inferiority, second-class citizenry and assumptions of criminality, microinvalidations, exoticization/assumptions of similarity, environmental microaggressions, and workplace and school microaggressions. Nadal defined each category as follows:

- Assumptions of Inferiority: Assumptions of inferiority manifest when people are assumed less intellectually capable, more impoverished or second-class because of belonging to a specific group (Sue et al., 2008). Marginalized and oppressed people develop feelings of inferiority that can take different nuances. In the case of Indigenous students, they might experience emotions such as: being less capable to perform academically, feeling less intelligent than others, considering themselves as not deserving high quality education because of their race, not being able to get a well-paid job or position in the university, being acknowledged as low income, or underclass.

- Second-class citizenry and assumptions of criminality: People are alleged as dangerous, criminal, or deviant for the sake of their look, phenotype, or race. These assumptions may manifest as body language or actions that show rejection or avoidance towards people. Behaviors that convey messages of devaluing or undermining others are also common (Sue et al., 2008).

- Microinvalidations: Microinvalidations are verbal comments that despite having good intentions, carry adverse messages to people denying their feelings or minimizing them. Microinvalidations are also defined as acts that exclude or 
negate people's thoughts, feelings or opinions. Microinvalidations manifest when perpetrators do not acknowledge their behavior as a manifestation of racism (Sue et al., 2008).

- Exoticization/assumptions of similarity: People receive comments regarding language, outfits, food or customs associated with a cultural background (Nadal, 2011). In particular, this exoticization might result in Indigenous students feeling embarrassed about using their home language in front of their peers. They may also hide their customs or believes in avoiding uncomfortable questions.

- Environmental microaggressions: This type of microaggressions is systemic and manifests at the macro level (Sue et al., 2007). Some examples of this macro level of hostilities are the curriculum not including the culture and contributions made by groups that have been excluded, as well as voices of the marginalized being silenced by a false notion of impartiality and fairness. Potential results include minorities being invited to prominent positions that let them succeed. Environmental microaggressions are challenging to identify as the system continually disguises the reality of oppression.

- Workplace and school microaggressions: Either in the workplace or at school hostilities can contribute to creating a negative environment resulting in augmenting dropout and desertion rates in students. An unfriendly atmosphere, overlooking people's opinions, treating people differently because of their ethnicity or ignoring others' needs are some examples of everyday microaggressions in the workplace (Nadal, 2011). 


\section{Possible Problems with REMS and Ethical Issues}

In implementing Nadal's (2011) Racial and Ethnic Microaggressions Scale in Ecuadorian contexts, a number of difficulties were anticipated. First, Nadal's original version was designed with consideration for specific characteristics and interactions of US colleges where students are more familiarized and informed of social issues. United States contexts are to some extent supportive and responsive to debate on racial topics. This openness may make it easier for college students to take a survey that requires them to elaborate on concepts related to racism. Although in Ecuador racism is prevalent, Beck, Mijeski, and Stark (2011) highlighted that around 70\% of Ecuadorians do not know what racism involves or what racial prejudice looks like. Even more remarkably, they revealed that Indigenous people "are the least aware of racism, discrimination, and prejudice" (p. 120). There was concern that this lack of awareness about racism might hinder Indigenous students' ability to understand the statements in the survey. In this regard, the original version of the REMS was adapted with vocabulary that was accessible to students before its application.

Second, the original version of the survey is in English. I followed a process of back translation for the REMS survey. The instrument was back-translated by a separate party who did not have affiliation with the study. The three steps of back translation recommended by Son (2008) were appropriate to ensure an adequate treatment of the sensitive topic, minimize language mistakes and assure quality. Back translation is a procedure recommended for researchers working with sensitive or high value content and has been used for survey research for more than 50 years (Son, 2018). As a translation technique, back translation involves three stages. First, the survey is translated from the 
original language into the target language. In a second phase, another person translates the target survey back into the source language. Finally, both the original and backtranslated versions are compared to modify the target language instrument until accuracy is properly achieved (McKay et al., 1996). It is advisable that the back translator does not have any previous knowledge regarding the original content of the document to give the translation more accuracy (Son, 2008). Because of language differences, a back translated document will never produce a $100 \%$ correspondence to the original text. However, the procedure helps identify misunderstandings, doubts, or language errors. Back translation is a suitable tool for researchers interested in translating questionnaires and surveys across cultures. Another advantage of back translation is that researchers can assert that their questions are as clear and exact as possible. In this sense, back translation is a valid option because it provides direct control over the quality of the content (Ozoling, 2009). The current study used back translation of the REMS from English to Spanish.

Ethical issues were important to consider in the application of the REMS survey. Any major moral constraint was directly associated with this study. However, given the topic is perceived as sensitive, I believed that some ethical principles had to be considered along with the process of surveying participants. Confidentiality was essential for protecting participants and animating them toward providing responses that accurately reflect their opinions. Throughout the process, I paid attention to aspects such as showing respect for respondents' freedom to participate, decision-making, and dignity. See REMS original version by components (Appendix A) and REMS survey randomized items in one category (Appendix E). 


\section{Research Question Two. What is the Association between Microaggressions and Indigenous Students’ Self-esteem?}

Self-esteem has been widely studied by using different measures available for psychologists, teachers, and counselors. In reviewing the literature, the most frequently scale used is the Rosenberg Self-Esteem Scale (RSES). This scale presents several advantages for researchers interested in having an overall estimate of people's selfesteem.

Conditions such as conciseness of the scale that includes only ten statements, easy to understand vocabulary even for people with low literacy levels, validity and reliability have made the RSES the tool of choice for use with different populations (Sinclair et al., 2010). RSES was developed in 1965, and since then it has been used in a variety of contexts with varying purposes including predicting delinquent behaviors, pregnancy in adolescents, drug abuse, and suicidal behaviors. RSES as a unitary self-administered scale to measure self-esteem has reported good levels of consistency with values of 0.85 for the scale (Bagley, Bolitho, \& Bertrand, 1997). Throughout the years, the scale has been translated into different languages. (Please see Appendix F for Rosenberg SelfEsteem Scale).

Albo et al., (2007) turned the RSES into Spanish language and applied it to 420 Spanish university students. Results of this study suggested that the unidimensional structure of the instrument proposed by Rosenberg has appropriate levels of internal consistency, stability and test-retest correlation that supported the reliability of the scale. This evidence justified the application of the instrument in a university context to assess self-esteem (Albo et al., 2007). Rojas, Zegers, and Foster (2009) determined the 
reliability and validity of Rosenberg Self-Esteem Scale in a sample of 473 young adults, adults, and seniors evenly distributed by gender, age, educational level, and income in Chile. The study concluded that the RSES fulfills validity and reliability criteria to measure general self-esteem in the view that the results obtained from the population were similar to those in other contexts where the instrument has been applied.

In comparing Albo et al (2007) and Rojas et al (2009), the wording of the latter is more precise and refers more to the way Ecuadorian people use the Spanish language. The instrument includes a short introduction for survey takers to know about the purpose of the survey as well as instructions on how to provide answers. These characteristics make Rojas' instrument more user friendly.

The Rosenberg Self-Efficacy Scale consists of 10 items that are measured on a 3point Likert scale from 0 to 3 as follows: $0=$ strongly disagree, $1=$ disagree, $2=$ agree, and $3=$ strongly disagree. The higher the score, the higher the self-esteem of the respondent. Scores between 15 and 25 are within normal range; scores below 15 suggest a low self-esteem level. Given the advantages of Rojas et al.'s (2009) adaptation of Rosenberg's original version of the self-esteem scale, this instrument was used with the present study. Table 4 shows assigned values for the scale.

\section{Table 4}

Rosenberg's Self-Esteem Scale Scoring

\begin{tabular}{cl}
\hline Assigned Score & Value \\
\hline 3 & Strongly Agree \\
2 & Agree \\
1 & Disagree \\
0 & Strongly Disagree \\
\hline
\end{tabular}




\section{Research Question Three. What is the Association between Microaggressions and Indigenous Students' Self-efficacy?}

Schwarzer and Jerusalem (1995) developed the General Self-Efficacy Scale (GSE) to measure people's perceived self-efficacy. The concept of perceived selfefficacy was theorized as the positive self-belief that an individual could complete a challenging task or deal with difficulty in diverse areas of human activity (Schwarzer and Jerusalem, 1995). The instrument is not limited to predicting how people handle daily problems but also their level of adaptation when experiencing different types of stressful life events. The survey can be applied to general adult individuals including adolescents. This self-administered scale has 10 items on a 4-point scale going from exactly true $=4$, moderately true $=3$, moderately untrue $=2$, and not at all true $=1$. The total score is calculated by finding the sum of all the items with a higher score indicating more selfefficacy. Schwarzer and Jerusalem (1995) created a broader questionnaire for selfefficacy, but the most widely used is the short version with ten statements (Please see Appendix G for General Self-Efficacy Scale).

The self-efficacy survey measures people's positive resistance as a resource factor. This brief, easy instrument has gained relevance because it operationalizes the construct of self-efficacy as related to subsequent behaviors that people manifest when facing hassle situations. Schwarzer and Jerusalem report that the criteria of validity and reliability of this scale were tested with high internal consistency ratings ranging from 0.82 to 0.93 . Regarding reliability, the instrument reached 0.47 and 0.63 for men and women respectively. Individuals scoring high in the questionnaire are people with positive emotions, optimistic and report good levels of work satisfaction. On the contrary, 
people with low scores in self-efficacy are more prone to depression, apprehension, stress, fatigue, and health problems (Schwarzer and Jerusalem, 1995).

Brenlla et al., (2010) tested criteria for validity and reliability of the Argentinian General Self-Efficacy Scale adaptation. A total of 292 individuals took the survey. Results showed 0.76 reliability, significant internal consistency, and validity suggesting that this instrument is appropriate to assess perceived self-efficacy. This version of the General Self-Efficacy Scale was used in this study. Table 5 indicates Self-Efficacy Scale values and scoring. Please see Appendix G for representation in English and Spanish of General Self-Efficacy Scale and how it was presented to participants.

\section{Table 5}

Schwartzer and Jerusalem's General Self-Efficacy Scale Scoring

\begin{tabular}{cl}
\hline Assigned Score & Value \\
\hline 4 & Exactly true \\
3 & Moderately true \\
2 & Moderately untrue \\
1 & Not at all true \\
\hline
\end{tabular}

\section{Qualitative Component}

The qualitative component of the current mixed methods study involved interviewing Indigenous undergraduate students from the target population. Interviews were conducted because, as Seidman (2013) notes, as a researacher, I am interested in understanding the lived experiences of Indigenous students with microaggressions and the meaning they make of that experience. Creswell (2014) stated that interviews are useful instruments when it is not possible for the researcher to observe participants. It is also suitable when there is interest in controlling the line of questioning to obtain 
evidence that is more comprehensive. The main advantage that interviews offered was that in their narratives, participants provided unique information that helped understand how they perceive microaggressions. Creswell (2014) indicated that interviews also offer a more customized and natural approach that supports a thorough exploration of participants' experience. Creswell's advantages for interviews are summarized in Galleta (2013) who concluded that the purpose of the interview is for the researcher to get an indepth understanding of the issue under analysis.

A semistructured interview was applied to Indigenous students who volunteered. Galletta (2013) described semistructured interviews as instruments that include both open-ended and more theoretically driven questions to stimulate participants to draw answers from their experience. The reasons for using a semistructured questionnaire for this study were multiple. Advantages for researchers using semistructured tools are that they give participants the opportunity to delve into their experiences and provide more details of their thoughts, attitudes, feelings, understandings, or beliefs. A semistructured questionnaire also allows the researcher to better access to participants' true feelings about the problem under analysis. Additional items also appear spontaneously during the free-flowing conversation and contribute to a better understanding of the topic. However, researchers must be aware that openness requires them to be properly prepared in their knowledge of the issue because unexpected answers can come from participants (Galletta, 2013).

Additionally, using semistructured instruments to interviewing is flexible and allows both researcher and interviewee to deliberate a theme in more detail. It also facilitates the provision of stimuli from the researcher to encourage the participant to 
elaborate on the answer with follow-up, why, or how questions. Furthermore, a semistructured interview is an option when unexpected responses arise during the interview so the researcher can change some questions during the interview to get information that contributes to clarify the topic. As for disadvantages, semistructured interviews are time-consuming and require the researcher to be knowledgeable on the subject and experienced in conducting interviews (Galletta, 2013).

The interview process in the study considered it was paramount to observe principles of building equity in the process of interviewing. Seidman (2013) defined equity in the interviewing process as "a balance between means and ends, between what is sought and what is given, between process and product and a sense of fairness and justice that pervades the relationship between participant and interviewer" (p. 111). Seidman (2013) explained equity as the researcher giving voice to people's stories, being explicit about the purposes of the research, providing participants with written consent that clarify responsibilities and expectations, and scheduling time for the interview that is appropriate for participants. Although equity principles stated above are crucial, it is in the interview where researchers need to observe equity. In addition, Seidman (2013) reminded researchers to avoid invading participants' space and induce answers founded on their preconceptions.

Another aspect of equity deals with being cautious of hurting participants' sensitivity with questions that may affect their emotional and mental health. Equity in interviewing means to focus interest on the participants' experience because their words need to be valued. Finally, Seidman (2013) recommended that researchers honor the dignity of participants. 


\section{Qualitative Sampling and Participants}

Purposive sampling was suitable mainly because the study focused on participants who share similar characteristics in terms of ethnicity and culture and who have experienced the central phenomenon (Creswell, 2008). Purposive sampling is recommended when researchers need to select candidates who satisfy qualities associated with the topic of the study (Etican, Abubakar, \& Sunusi, 2016). The main advantage of purposive sampling in my study was that it did not constrain limit the number of participants for the study but to choosing participants according to what needs to be addressed in the study. Therefore, participants were met mainly by their knowledge and experience.

The demographic information collected in the survey was used to identify potential participants. Selection conditions required students to identify themselves as Indigenous, preferably those who were in their sophomore, junior, or senior year, since they had more exposure to campus life and had already interactions with teachers, administrators, and peers.

There were no restrictions to age with respect to choosing participants. Regarding the number of students answering the semistructured questionnaire, I secured a sample size that was large enough to appropriately depict the phenomenon of interest and address the research questions. The goal was to interview as many participants as necessary to add new perspectives to the analysis of microaggressions. Bowen (2008) recommends that researchers ponder the point when no new information is discovered. This point was reached when interviews yielded similar results across participants. 


\section{Qualitative Component Rigor}

Guba (1981) provided a set of criteria for assuring the trustworthiness of findings when using naturalistic methods. Four aspects of integrity are important to consider in qualitative research: truth-value, applicability, consistency, and neutrality.

The current study found the element of truth-value (internal validity) as the most salient condition to achieve trustworthiness. Truth-value in naturalistic terms is analogous to the credibility of findings and interpretations (Guba, 1981). There are several strategies researchers can use to give their studies credibility. Having a prolonged engagement at the research site, using persistent observation, doing peer debriefing, doing triangulation, collecting referential adequacy materials and doing member checks (Guba 1981).

For the current study, member checks was an option to assure credibility. Member checks according to Guba (1981) is "the single most important action inquirers can take, for it goes to the heart of the credibility criterion" (p. 85). Member check or participant validation is defined as a quality assurance process through which researchers strive for improving accuracy, credibility, and validity of what has been documented during the research interview procedure (Doyle, 2007). In the case that participants confirmed the accuracy and completeness of the information, the study is recognized as credible (Harper \& Cole, 2012).

By using member checking, participants of my study had the opportunity to review if the researcher adequately understood their thoughts, ideas, and opinions. The approach to conduct member checking consisted of the researcher restating information given by the participant and then asking if what was summarized was accurate to his/her intentions, views, feelings, and experiences. The study adopted member checking 
because of time and accessibility constraints. The member checking strategy was used with five participants whose answers were difficult to interpret. Additional feedback from participants was necessary to clarify their ideas for one question in the understanding of racial microaggressions and perception of racialized environment domain and two questions in the coping with racial microaggressions domain respectively. The researcher contacted the participants via Zoom and worked with them individually. The procedure started with the researcher presenting the students the transcript of the answers they provided. Students were given some time to validate their answers. The following step was to restate the participant's answers and then asking them to confirm the accuracy of the researcher's interpretation.

\section{Qualitative Data Analysis}

The lived experiences of Indigenous students with microaggressions were examined. To be able to capture participants' experiences, the researcher adopted Prus' (1996) approach to qualitative data analysis. The author suggests researchers to involve in a process to profoundly understand students' thoughts, feelings, and actions. My involvement as a researcher was necessary to enter the participants' world with depth, openness, and appreciation of their experience. Given the interaction the researcher had with Indigenous students, their experiences needed to be carefully analyzed and in search for meaning (Prus, 1996). The underlying meaning of students' accounts was examined with emotion coding. Saldaña (2009) recommended the use of affective methods, otherwise known as emotion coding, to delve into the cognitive and subjective systems of participants. 
Saldaña argues that emotion coding is suitable for a variety of qualitative studies but specifically for those exploring intrapersonal and interpersonal experiences and actions. Emotion coding is adequate when researchers want to understand the feelings of participants from a particular experience in their lives. Researchers using emotion coding label the recalled emotions of participants and make inferences from those expressed feelings. Saldaña emphasizes that emotions are inherent to any human experience and that understanding them provides "deep insight into the participants' perspectives, worldviews, and living conditions" (p. 86). Researchers' ability to infer emotions lived by participants is crucial to have a clear understanding of the phenomenon under analysis. In this study, Indigenous students' exposure to microaggressions needed to be examined from the perspective of their emotional responses, since it was the researcher's interest to understand their feelings that ultimately affect their campus life experience.

Qualitative data analysis began by a careful process of reviewing the audio recordings. The researcher assigned a number to the recordings in the order they were collected. Once the recordings were organized, the researcher listened to the audios searching for poor sound quality, accidental pauses and lack of clarity resulting from external disruptions during the interviews. The researcher determined that environmental noises and disruptions that arose in some interviews did not hinder the quality of the audio recordings. Then, the researcher listened to the audios once again and started taking notes for preliminary ideas and thoughts. In the next step, I transcribed each interview. Once the interviews were transcribed, the researcher read each participant's transcript. This initial reading was necessary to take notes and have a clear notion of participants' experiences. Reading each transcript in its entirety provided a broad sense of the data 
and to reflect on its overall meaning (Creswell, 2009). In a second round of reading, the researcher looked at the information thoroughly watching for excerpts that captured students' encounters with microaggressions. A third reading identified the most outstanding quotes that gave shape to the tentative codes that emerged from the raw data. Identifying quotes represented what Creswell (2009) calls the heart of this qualitative portion of the study. The codes led to the identification of themes that were used to write the narrative that resulted from data exploration.

\section{Research Question Four. How do Indigenous Students Experience Racial Microaggressions while Pursuing a Degree in a Public Higher Education Institution on Issues of Responses to Microaggressions, and Mechanisms they use to cope with \\ Microaggressions?}

I interviewed each student twice. The first interview lasted approximately 30 minutes. The purpose of the first interview was to grasp students' life experiences in the context they lived in by asking them to provide details about themselves, their families, their place of origin, their school experience, the nature of their relationships between teachers, peers. In this first interview, I also asked them how respected, visible and supported they feel in their institution. The first step in the interview process was crucial because it created a sense of confidence between the researcher and the interviewees. In addition, their answers let me take some initial notes and follow-up questions that I used in the second interview.

The second interview included questions that provided information on how aware students were regarding microaggressions. The second interview lasted about 45 minutes. In the second interview, I used an elicitation technique to help students bring their own 
lived experiences to scene and make connections to the topic. I asked students to read stories of people who have suffered racial hostilities. While reading, students were asked to evoke their own experiences in similar situations. I used this elicitation technique to help interviewees have a clear idea of microaggressions and situate their experience within the flow of the interview. The use of the elicitation technique was an asset since it not only anchored students' experience and facilitated the conversation but also helped to reduce tension during the interview.

\section{Themes Addressed in the Qualitative Interview}

The qualitative component of the study consisted of a semistructured interview to give voice to Indigenous students who depicted their experiences with racial microaggressions. While the interview for this mixed-method study answered research question 4, it also aimed at enhancing the information obtained in the quantitative stage.

The interview protocol consisted of four domains. Each domain had a leading question with subsequent follow-up questions. Domain one, participants were asked to provide details of their personal and family background. Personal information was necessary to get in-depth knowledge of participants' place of origin, residence, career choices, previous school encounters, current college experience, and the nature of interactions with teachers, peers, and administrative. Domain two addressed participants' understanding of racial microaggressions and their perceptions of the racialized environment. Students were introduced to the concept of microaggressions by presenting a prompt that helped them recall similar racial assaults they may have suffered. Students were asked to reflect on the concept of microaggressions and to report their feelings when being targeted by a racial hostility. Additionally, students were asked to describe the 
campus environment in relation to their positive or negative experiences as Indigenous students when interacting with Mestizo people. Students also explained the extent to which they believed their institution was culturally welcoming. They were required to gauge if they considered the curriculum contributed to the understanding and validation of their culture. In domain three, students explained their responses to microaggressions. Students described their initial feelings or thoughts when they believed they had been racially attacked and the corresponding response it generated in them. Finally, domain four involved descriptions of the coping mechanisms Indigenous students adopt to protect themselves and diminish the adverse impact of microaggressions. Table 6 summarizes the domains in the interview protocol and leading statements. Please refer to the complete interview protocol in English and Spanish (Appendix H) and the adult consent form to participate in the study in both languages (Appendix I).

Table 6

Racial Microaggressions Semistructured Interview Domains and Leading Statements

\begin{tabular}{ll}
\hline Domain & Leading statement \\
\hline Getting to know the participant & $\begin{array}{l}\text { Provide details of your personal and family } \\
\text { background information }\end{array}$ \\
$\begin{array}{l}\text { Understanding of racial } \\
\text { microaggressions and perception of } \\
\text { racialized environment }\end{array}$ & $\begin{array}{l}\text { Definition of racial microaggressions. } \\
\text { Example. }\end{array}$ \\
$\begin{array}{l}\text { Responses/reactions to } \\
\text { microaggressions }\end{array}$ & $\begin{array}{l}\text { Description of a racialized environment. } \\
\text { Coping with racial microaggressions }\end{array}$ \\
$\begin{array}{l}\text { Description responses/reactions when being } \\
\text { targeted by a racial slur. }\end{array}$ \\
$\begin{array}{l}\text { Description of different strategies used by } \\
\text { participants to address microaggressions }\end{array}$ \\
\hline
\end{tabular}




\section{Chapter Summary}

This chapter presented an overview of the methods used in this study to examine racial microaggressions. The chapter started with a comparison between positivist and naturalistic approaches followed by the rationale for adopting mixed methods in the form of sequential transformative approach. Finally, both quantitative and qualitative components were described in detail including descriptions of surveys, semistructured interview, sampling, participants, data collection and data analysis. 


\section{CHAPTER FOUR}

\section{RESULTS}

This current mixed methods study examined microaggressions of undergraduate Indigenous students in a public higher education institution in Ecuador. The purpose of this chapter is to present the findings according to the quantitative and qualitative data collected in the study. The following information describes the type of microaggressions Indigenous students identified as happening in their lives, the association between microaggressions and students' self-esteem, the association between microaggressions and students' self-efficacy and the experience students have regarding microaggressions on aspects such as responses and mechanisms they use to cope with hostilities. The current study addressed the following research questions:

1. What are the most/least common types of racial microaggressions that Indigenous students experience while pursuing a degree in a public higher education institution?

2. What is the association between microaggressions and Indigenous students' self-esteem?

3. What is the association between microaggressions and Indigenous students' self-efficacy?

4. How do Indigenous students experience racial microaggressions while pursuing a degree in a public higher education institution on issues of (a) responses to microaggressions, and (b) mechanisms they use to cope with microaggressions? 
The results and findings of this chapter are divided into two components. The first component comprises quantitative results from the demographic information of participants, racial microaggressions survey, associations between microaggressions and self-esteem, and associations between microaggressions and self-efficacy. The results of this component deal with research questions one, two, and three. The second component includes the qualitative results from the semistructured interview containing a brief introduction of the interviewees, an account of their experiences with microaggressions, and a description of their responses/reactions and coping mechanisms they use to deal with racial hostilities. This component answers research question four.

\section{Component One: Quantitative Study Results}

\section{Participants' Demographic Information and Descriptions}

This first section includes demographic information of respondents in this study for both quantitative and qualitative components. One hundred and twelve Indigenous students attending a public higher education institution consented to participating in the quantitative component of this study. The sample consisted of $50 \%$ males and $50 \%$ females ranging in age from 18 to 26 years old with the average being 20.7 years of age. Eighty-nine respondents (79.5\%) identified as single, $22(19.6 \%)$ identified as married, and only $1(0.9 \%)$ identified as divorced. In reporting wages, $71(63.4 \%)$ of the respondents said they live on an income that goes from $\$ 50$ to $\$ 200^{5}$. Thirty-eight (33.9\%) had an income that ranged from $\$ 201$ to $\$ 350$ that still does not cover the basic needs of a low-income family. Only three students $(2.7 \%)$ had an income that is enough

\footnotetext{
${ }^{5}$ In 2000, the Ecuadorian Congress passed the law to replace the Sucre with the United States Dollar. Since then, the U.S. Dollar is the official currency in Ecuador.
} 
to subsidize basic needs. Regarding the place of residence, 45 (40.2\%) of the respondents live in town while 67 (59.8\%) live in communities located outside town. For housing, 37 participants (32\%) live with their parents while $56(50 \%)$ rent a place. Participants who responded "other," 19 (17\%), indicated they cohabited with friends or close relatives. As for employment, 4 (3.6\%) of the respondents said they are employed in the private sector. Three $(2.7 \%)$ work for private businesses in the city. Only one student $(0.9 \%)$ had a job in the public sector. Seven students (6.3\%) had established their own small business as a means of earning their living. Twenty-one (18.8\%) of respondents were unemployed or looking for a job. Thirteen (11.6\%) students worked as housekeepers and other domestic tasks. Thirty-three (29.5\%) respondents had occasional jobs where they did not have any expectation to be hired in the future times. Twenty-two (19.6\%) students did not report any activity as a source of income. Finally, eight (7.1\%) who endorsed other as an employment type, indicated they had sporadic jobs such as parade dancers, gardeners, housekeepers, and taxi drivers. Table 8 shows demographic characteristics of participants.

Of the 112 respondents, all identified as Indigenous. Forty-two (37.5\%) reported speaking Spanish exclusively while 70 (62.5\%) spoke both Spanish and Indigenous language identified as Quichua. In terms of written languages, 83 (64\%) responded they were able to write in Spanish only while twenty-nine (25.9\%) reported being able to write in both Spanish and Quichua languages. Twenty participants (17.9\%) responded their parents speak Spanish as their main language while ninety-two (82.1\%) said their parents speak Spanish and Quichua. 
Finally, for parents' educational attainment, eighty-seven $(77.7 \%)$ responded that their parents completed primary school. Twenty-three (20.5\%) said their parents completed secondary school. Only two $(1.8 \%)$ responded that their parents completed college and obtained a degree. Table 7 displays a summary of respondents' demographic information.

\section{Table 7}

Summary of Self-Reported Demographic Information $(N=112)$

\begin{tabular}{|c|c|c|}
\hline Categorical Demographic Variable & Frequency & Percent \\
\hline \multicolumn{3}{|l|}{ Gender } \\
\hline Male & 56 & $50 \%$ \\
\hline Female & 56 & $50 \%$ \\
\hline \multicolumn{3}{|l|}{ Age } \\
\hline $18-20$ & 50 & $44.6 \%$ \\
\hline $21-23$ & 41 & $36.6 \%$ \\
\hline $24-26$ & 18 & $16.1 \%$ \\
\hline 26 and beyond & 3 & $2.7 \%$ \\
\hline \multicolumn{3}{|l|}{ Marital Status } \\
\hline Single & 89 & $79.5 \%$ \\
\hline Married & 22 & $19.6 \%$ \\
\hline Divorced & 1 & $0.9 \%$ \\
\hline \multicolumn{3}{|l|}{ Separated } \\
\hline \multicolumn{3}{|l|}{ Monthly Income } \\
\hline$\$ 50-200$ & 71 & $63.4 \%$ \\
\hline$\$ 201-350$ & 38 & $33.9 \%$ \\
\hline$\$ 351-500$ & 3 & $2.7 \%$ \\
\hline \multicolumn{3}{|l|}{$\$ 501-650$} \\
\hline \multicolumn{3}{|l|}{ Residence } \\
\hline In town & 45 & $40.2 \%$ \\
\hline Outside town & 67 & $59.8 \%$ \\
\hline \multicolumn{3}{|l|}{ Housing } \\
\hline Parents with mortgage & 11 & $9.8 \%$ \\
\hline Parents without mortgage & 26 & $23.2 \%$ \\
\hline Rented & 56 & $50 \%$ \\
\hline Other & 19 & $17 \%$ \\
\hline
\end{tabular}




\section{Employment type}

Employed private sector $\quad 4 \quad 3.6 \%$

$\begin{array}{lll}\text { Private business } & 3 & 2.7 \%\end{array}$

Employed public sector $\quad 1 \quad 0.9 \%$

$\begin{array}{lll}\text { Own a business } & 7 & 6.3 \%\end{array}$

Unemployed and looking for a job $\quad 21 \quad 18.8 \%$

Household $\quad 13 \quad 11.6 \%$

Occasionally Employed $\quad 33 \quad 29.5 \%$

None $\quad 22 \quad 19.6 \%$

$\begin{array}{lll}\text { Other } & 8 & 7.1 \%\end{array}$

\section{Race and Ethnicity}

Indigenous

112

$100 \%$

Mestizo

Mulato

Afro Ecuadorian

Other

\section{Languages}

Spanish only

$42 \quad 37.5 \%$

Spanish and Indigenous Language

$70 \quad 62.5 \%$

Other

\section{Languages written}

Spanish only

$83 \quad 64 \%$

Spanish and Indigenous Language

29

$25.9 \%$

Other

\section{Languages spoken by parents}

Spanish only

Spanish and Indigenous Language

Other

\section{Parents' level of education}

Primary completed

Secondary completed

University completed with a degree

Other

$\begin{array}{cl}87 & 77.7 \% \\ 23 & 20.5 \% \\ 2 & 1.8 \%\end{array}$

The quantitative portion of this mixed methods study used three scales to answer the first three research questions. The Racial and Ethnic Microaggressions survey 
(REMS) assessed the most/least types of microaggressions perceived by students. The Rosenberg Self-Esteem Scale (RSES) measured students' global self-worth by measuring their positive and negative feelings about themselves. The General Self-Efficacy Scale (GSE) determined participants' perceptions of their capacity to achieve goals and perform tasks. What follows is the results for quantitative and qualitative research components for this mixed-methods study.

\section{Research Question One: What are the Most/Least Common Types of Racial} Microaggressions that Indigenous Students Experience while Pursuing a Degree in a Public Higher Education Institution?

The first question in this study aimed at determining the occurrence of racial microaggressions in daily the daily lives of Indigenous students. To achieve this aim, 112 Indigenous undergraduate students took the Racial Microaggressions Survey (REMS). The scale measured the types of microaggressions that individuals experience in their everyday lives (Nadal, 2011). REMS is a survey that utilizes Likert-type scale to rate the incidence of an event over a six-month period for each 45 questions divided in six components. The mean for every statement corresponds to the average of occurrences in the last six-months according to participants' perceptions. Table 8 displays the descriptive statistics, mean and standard deviation for all items in the survey arranged by components. 
Table 8

Occurrence of Microaggressions. Means and Standard Deviations of REMS Components.

\begin{tabular}{|c|c|c|c|}
\hline & Students' Perceptions & $M$ & $S D$ \\
\hline & Component 1: Assumptions of Inferiority & & \\
\hline 32 & $\begin{array}{l}\text { Someone assumed that I would have a lower education } \\
\text { because of my race/ethnicity. }\end{array}$ & 3.42 & 1.05 \\
\hline 38 & $\begin{array}{l}\text { Someone assumed that I was poor because of my } \\
\text { race/ethnicity. }\end{array}$ & 3.53 & 1.08 \\
\hline 21 & $\begin{array}{l}\text { Someone assumed that I would not be educated because } \\
\text { of my race/ethnicity. }\end{array}$ & 3.26 & 1.00 \\
\hline 17 & $\begin{array}{l}\text { Someone acted surprised at my scholastic or } \\
\text { professional success because of my race/ethnicity. }\end{array}$ & 2.58 & 1.09 \\
\hline 9 & $\begin{array}{l}\text { Someone assumed that I would not be intelligent } \\
\text { because of my race/ethnicity. }\end{array}$ & 3.18 & 1.00 \\
\hline 36 & $\begin{array}{l}\text { Someone assumed that I held a lower paying job } \\
\text { because of my race/ethnicity. }\end{array}$ & 2.91 & 0.95 \\
\hline 5 & $\begin{array}{l}\text { Someone assumed that I grew up in a particular } \\
\text { neighborhood because of my race/ethnicity }\end{array}$ & 3.35 & 0.96 \\
\hline 22 & $\begin{array}{l}\text { Someone told me that I was "articulate" after she/he } \\
\text { assumed I would not be. } \\
\text { Component 2: Second-Class Citizenry and } \\
\text { Assumptions of Criminality }\end{array}$ & 2.63 & 0.97 \\
\hline 6 & $\begin{array}{l}\text { Someone avoided walking near me on the street because } \\
\text { of my race/ethnicity. }\end{array}$ & 1.96 & 0.86 \\
\hline 31 & $\begin{array}{l}\text { Someone clenched her/his purse or walled upon seeing } \\
\text { me because of my race/ethnicity. }\end{array}$ & 1.81 & 0.71 \\
\hline 8 & $\begin{array}{l}\text { Someone avoided sitting next to me in a public space } \\
\text { (e.g., restaurants, movie theaters, subways, buses) } \\
\text { because of my race/ethnicity. }\end{array}$ & 2.16 & 0.81 \\
\hline 40 & $\begin{array}{l}\text { Someone avoided eye contact with me because of my } \\
\text { race/ethnicity. }\end{array}$ & 1.88 & 0.84 \\
\hline 2 & $\begin{array}{l}\text { Someone's body language showed they were scared of } \\
\text { me, because of my race/ethnicity. }\end{array}$ & 2.37 & 1.22 \\
\hline 34 & $\begin{array}{l}\text { Someone assumed that I would physically hurt them } \\
\text { because of my race/ethnicity. }\end{array}$ & 1.46 & 0.59 \\
\hline 11 & $\begin{array}{l}\text { I received substandard service in stores compared to } \\
\text { customers of other racial groups. } \\
\text { Component 3: Microinvalidations }\end{array}$ & 2.77 & 0.94 \\
\hline 27 & Someone told me that they "don't see color." & 3.54 & 0.90 \\
\hline 30 & Someone told me that they do not see race/ethnicity. & 3.26 & 0.90 \\
\hline 39 & $\begin{array}{l}\text { Someone told me that people should not think about } \\
\text { race/ethnicity anymore. }\end{array}$ & 3.33 & 0.94 \\
\hline 7 & Someone told me that they were color-blind. & 3.27 & 0.97 \\
\hline 26 & $\begin{array}{l}\text { I was told that people of color do not experience racism } \\
\text { anymore. }\end{array}$ & 3.49 & 0.91 \\
\hline
\end{tabular}


33 Someone of a different racial group has stated that there is no difference between the two of us.

4 I was told that I should not complain about race/ethnicity.

14 I was told that people of all racial/ethnic groups experience the same obstacles.

10 I was told that I complain about race too much.

Component 4: Exoticization/Assumptions of Similarity

3 Someone assumed that I spoke a language other than Spanish because of my race/ethnicity.

29 Someone asked me to teach them words in my "native language."

$3.19 \quad 0.98$

45 Someone assumed that I speak similar languages to other people in my race.

35 Someone assumed that I ate foods associated with my race/culture every day.

42 Someone told me that all people in my racial group look alike.

23 Someone told me that all people in my racial group are all the same.

13 Someone wanted to date me only because of my race.

20 Someone did not believe me when I told them I was born in the U.S.

43 Someone objectified one of my physical features because of my race/ethnicity.

\section{Component 5: Environmental Microaggressions}

37 I observed people of my race/ethnicity portrayed positively in movies.

24 I observed people of my race/ethnicity portrayed positively in magazines.

19 I observed people of my race/ethnicity portrayed positively on television.

28 I read popular books or magazines in which a majority of contributions featured people from my racial group.

18 I observed that people of my race/ethnicity were the CEOs of major corporations.

41 I observed that someone of my race/ethnicity is a government official in my state.

12 I observed people of my race/ethnicity in prominent positions at my workplace or school.

\section{Component 6: Workplace and School Microaggressions}

25 An employer or co-worker was unfriendly or unwelcoming toward me because of my race/ethnicity. 


\begin{tabular}{llcc}
\hline 15 & $\begin{array}{l}\text { My opinion was overlooked in a group discussion } \\
\text { because of my race/ethnicity. }\end{array}$ & 2.71 & 0.91 \\
1 & $\begin{array}{l}\text { I was ignored at school or at work because of my } \\
\text { race/ethnicity. }\end{array}$ & 2.56 & 1.01 \\
16 & $\begin{array}{l}\text { Someone assumed that my work would be inferior to } \\
\text { people of other racial groups. }\end{array}$ & 3.16 & 1.08 \\
44 & $\begin{array}{l}\text { An employer or co-worker treated me differently than } \\
\text { White co-workers. }\end{array}$ & 2.76 & 0.84 \\
\hline
\end{tabular}

While Table 8 presents descriptive statistics for all the statements in every component in the survey, Table 9 condenses the mean and standard deviation for the six components separately. Assumptions of inferiority and microinvalidations displayed the highest means. Second-class citizenry and assumptions of criminality reported the lowest mean.

\section{Table 9}

Students' Perception of Occurrence of Microaggressions. Means and Standard Deviations Summary of Components

\begin{tabular}{lcc}
\hline Categorical Variable & Mean & $S D$ \\
\hline Component 1: Assumptions of Inferiority & 3.11 & 0.74 \\
Component 2: 2nd-Class Citizenry and & & \\
Assumptions of Criminality & 2.06 & 0.56 \\
Component 3: Microinvalidations & 3.09 & 0.60 \\
Component 4: Exoticization/Assumptions of Similarity & 2.72 & 0.59 \\
Component 5: Environmental Microaggressions & 2.46 & 0.63 \\
Component 6: Workplace and School Microaggressions & 2.86 & 0.75 \\
\hline
\end{tabular}

* Perceived Frequency of microaggression in a six-month span

\section{REMS Component One: Assumptions of Inferiority}

Overall, assumptions of inferiority reported the highest mean of all the components $(M=3.11)$. Students' answers indicated three microaggressions related to assumptions of inferiority as the most frequent "Someone assumed that I was poor 
because of my race/ethnicity" $(\mathrm{M}=3.53)$, "Someone assumed that I would have a lower education because of my race/ethnicity" $(\mathrm{M}=3.42)$, and "Someone assumed that I grew up in a particular place because of race/ethnicity" $(\mathrm{M}=3.35)$. The annotated means suggest that students perceived these events as occurring four-to-six times in the past six months. The lowest mean for this component was "Someone acted surprised at my scholastic or professional success because of my race/ethnicity" $(M=2.58)$. All the statements in the assumptions of inferiority component accounted means above one. REMS Component Two: Second-Class Citizenry and Assumptions of Criminality

Second-class citizenry and assumptions of criminality obtained the lowest mean of the six microaggressions components $(\mathrm{M}=2.06)$. However, students' perceptions revealed prevalent microaggressions related to this category. The three highest scores for this component were "I received substandard service in stores compared to customers of other racial/ethnic groups" $(\mathrm{M}=2.77)$, “Someone's body language showed they were scared of me because of my race/ethnicity" $(M=2.37)$, and "Someone avoided sitting next to me in a public spaces because of my race/ethnicity $(M=2.16)$. Results indicated that participants perceived the occurrence of these events one-to-three times in the past six months. The least common microaggression perceived by participants was "Someone assumed that I would physically hurt them because of my race/ethnicity" $(\mathrm{M}=1.46)$. According to participants' means responses, four subscales in the second class citizen and assumptions of criminality component had means ranging from $(\mathrm{M}=1.46$ to $\mathrm{M}=1.96)$ meaning that none of the participants perceived they had experienced these hostilities in the last six months. 


\section{REMS Component Three: Microinvalidations}

Microaggressions in the form of microinvalidations showed the second highest mean $(M=3.09)$ of the six components in REMS. Seven items in this component had means above three revealing that students perceived these events happening four-to-six times in the past six months. The microaggressions with the highest mean were "Someone told me that they "do not see color"" $(\mathrm{M}=3.54)$ followed by "I was told that people of color do not experience racism anymore" $(\mathrm{M}=3.49)$. "Someone of a different race/ethnicity believe that there is no difference between the two of us" $(\mathrm{M}=3.43)$ reported the third highest mean. Interestingly, two more items reported identical means $(\mathrm{M}=3.33)$ "I was told that people of color do not experience racism anymore" and "I was told that I should not complain about race." These means indicate that Indigenous students perceived these occurrences happening four-to-six times in the past six months. Finally, students' opinion about people of all racial groups experiencing the same obstacles endorsed the lowest mean $(M=2.04)$ indicating that participants perceived this racial microaggression affecting them one-to-three times in the past six months.

\section{REMS Component Four: Exoticization and Assumptions of Similarity}

The most endorsed statements within component four were "Someone assumed that I spoke a language other than Spanish because of my race/ethnicity" $(M=3.35)$, followed by "Someone asked me to teach them words in my native language" $(\mathrm{M}=3.19)$, and the statement "Someone objectified one of my physical features because of my race/ethnicity" $(M=3.08)$. Eight out of nine of the statements in this category showed means ranging from 3.35 to 2.58 , meaning that participants perceived these events occurring one-to-six times in the past six months. Interestingly, in component 
four, exoticization/assumptions of similarity, participants endorsed the statement "Someone wanted to date me only because of my race/ethnicity" as the lowest mean (M $=1.35$ ). This mean was in turn the lowest mean of all the 45 statements in the survey.

\section{REMS Component Five: Environmental Microaggressions.}

The most endorsed statement in this component was "I observed people of my race/ethnicity portrayed positively on television" $(\mathrm{M}=2.73)$ followed by the statement "I observed people of my race/ethnicity portrayed positively in magazines" $(\mathrm{M}=2.53)$. Respondents endorsed $(\mathrm{M}=2.51)$ to the subscale "I observed that people of my race/ethnicity were managers of major corporations." Finally, the statement "I read popular books or magazines in which a majority of contributions features people from my racial group" endorsed the lowest mean $(M=2.16)$. Means for the environmental microaggressions component were roughly uniform ranging from $(\mathrm{M}=2.73)$ to $(\mathrm{M}=$ 2.16) implying that students perceived people of their race/ethnicity being positively represented one-to-three times in the past six months.

\section{REMS Component Six: Workplace and School Microaggressions}

For the items in this component, students perceived "Someone assumed that their work is inferior compared to people of other racial groups" was the most common event they experience $(M=3.16)$. Respondents endorsed "An employer or co-worker was unwelcoming toward me because of my race/ethnicity" a mean of $(\mathrm{M}=3.11)$. Both means say that participants perceived these events occurring four-to-six times in the last six months. The lowest mean for this category according to respondents was "My opinion was overlooked in a group discussion because of my race/ethnicity" $(\mathrm{M}=2.71)$. 


\section{Research Question Two. What is the Association between Microaggressions and Indigenous Students' Self-esteem?}

A Pearson correlation coefficient was run to assess the relationship between microaggressions and Indigenous undergraduate students' self-esteem using SPSS version 2.0. Each component of the REMS was compared to students' self-esteem results. There was a significant negative correlation between the assumptions of inferiority reported by participants and their self-esteem $(\mathrm{r}=-.235, \mathrm{p}=.05)$ two-tailed. This result indicates that students' self-esteem decreases as they perceive being treated as inferior. The second class and assumption of criminality component was also significantly and negatively correlated with students' self-esteem $(r=-.209, \mathrm{p}=.05)$ two-tailed. The microinvalidations factor reported negative association with students' self-esteem but the relationship was not significant $(r=-.173, \mathrm{p}=.05)$ two-tailed. Exoticization and assumptions of similarity also showed a negative non-significant correlation. Environmental microaggressions showed a positive and significant correlation $(\mathrm{r}=.324$, $\mathrm{p}=.01)$ two-tailed. Component six, workplace and school microaggressions correlated negatively and significantly according to students' answers $(r=-.246, \mathrm{p}=.05)$ two tailed.

Overall, components one, two, and six showed a negative and significant correlation to students' self-esteem. Additionally, components three and four endorsed negative correlation but were not significant. Finally, component five reported positive and significant correlation. Table 10 summarizes correlation results for microaggressions and self-esteem. 
Table 10

REMS Correlations for Self-esteem.

\begin{tabular}{|c|c|c|c|c|c|c|}
\hline REMS Component & 1 & 2 & 3 & 4 & 5 & 6 \\
\hline 1. Assumption of Inferiority & 1 & & & & & \\
\hline $\begin{array}{l}\text { 2. Second Class and } \\
\text { Assumption of Criminality }\end{array}$ & $.761^{* *}$ & 1 & & & & \\
\hline 3. Microinvalidations & $.780^{* *}$ & $.703^{* *}$ & 1 & & & \\
\hline $\begin{array}{l}\text { 4. Exoticization and } \\
\text { Assumptions of Similarity }\end{array}$ & $.761^{* *}$ & $.799^{* *}$ & $.721^{* *}$ & 1 & & \\
\hline $\begin{array}{l}\text { 5. Environmental } \\
\text { Microaggressions }\end{array}$ & -.106 & .078 & .086 & .156 & 1 & \\
\hline $\begin{array}{l}\text { 6. Workplace and School } \\
\text { Microaggressions }\end{array}$ & $.856^{* *}$ & $.783^{* *}$ & $.761^{* *}$ & $.772^{* *}$ & -.097 & 1 \\
\hline $\begin{array}{l}\text { Self Esteem Sum per } \\
\text { Participant }\end{array}$ & $-.235^{*}$ & $-.209^{*}$ & -.173 & -.167 & $.324^{* *}$ & $-.246^{* *}$ \\
\hline
\end{tabular}

\section{Research Question Three. What is the Association between Microaggressions and}

\section{Indigenous Students' Self-efficacy?}

Likewise, the association between racial microaggressions reported by Indigenous undergraduate students' and their self-efficacy was assessed with Pearson correlation coefficient.

Results showed that there was a negative and significant correlation between component one assumptions of inferiority and self-efficacy $(r=-.380, p=.01)$ two tailed. This outcome, suggests that while occurrences related to assumptions of inferiority such as people assuming students live in poverty, have low education, or grow up in a particular place because of their race/ethnicity increase, students' self-efficacy decreases. In addition, results showed statistically significant negative relationship between the second component of the REMS named as second-class citizen and assumptions of criminality and students' self-efficacy $(r=-.382, \mathrm{p}=.01)$ two-tailed. The third component 
of the REMS referred as microinvalidations was also found as significant and negative correlated to self-esteem $(\mathrm{r}=-.250, \mathrm{p}=.01)$.

Based on the correlation analysis performed, we found out that there was a negative significant correlation between the exoticization and assumption of similarity component and students' self-efficacy $(r=-.332, p=.01)$ two-tailed. Environmental microaggressions were found to have a positive and significant relationship with selfefficacy $(r=-.363, p=.01)$ two-tailed. Finally, the data revealed a negative and significant relationship between workplace and school microaggressions and students' self-efficacy $(\mathrm{r}=-.463, \mathrm{p}=.01)$. In other words, the more perceived hostilities in the school environment, the lower self-efficacy levels for participants. Table 11 summarizes the information above.

\section{Table 11}

REMS Correlations for Self-efficacy.

\begin{tabular}{|c|c|c|c|c|c|c|}
\hline \multicolumn{7}{|l|}{$\begin{array}{l}\text { REMS Microaggressions } \\
\text { Component }\end{array}$} \\
\hline 1. Assumption of Inferiority & 1 & & & & & \\
\hline $\begin{array}{l}\text { 2. Second Class and } \\
\text { Assumption of } \\
\text { Criminality }\end{array}$ & $.761^{* *}$ & 1 & & & & \\
\hline 3. Microinvalidations & $.780^{* *}$ & $.703^{* *}$ & 1 & & & \\
\hline $\begin{array}{l}\text { 4. Exoticization and } \\
\text { Assumptions of Similarity }\end{array}$ & $.761^{* *}$ & $.799^{* *}$ & $.721^{* *}$ & 1 & & \\
\hline $\begin{array}{l}\text { 5. Environmental } \\
\text { Microaggressions }\end{array}$ & -.106 & .078 & .086 & .156 & 1 & \\
\hline $\begin{array}{l}\text { 6. Workplace and School } \\
\text { Microaggressions }\end{array}$ & $.856^{* *}$ & $.783^{* *}$ & $.761^{* *}$ & $.772^{* *}$ & -.097 & 1 \\
\hline $\begin{array}{l}\text { Self Esteem Sum per } \\
\text { Participant }\end{array}$ & $-.380^{* *}$ & $-.382^{* *}$ & $-.250^{* *}$ & $-.332^{* *}$ & $.363^{* *}$ & $-.463^{* *}$ \\
\hline
\end{tabular}




\section{Component Two: Qualitative Study Results}

\section{Participants' Introduction and Pseudonyms}

The qualitative component of this study required a smaller sample. Students who completed the survey were invited to express their interest in participating in the semistructured interview process. Seventeen students contacted the researcher, but only twelve set up an appointment for the interview. Interviewees' selection attended to the same four criteria for the quantitative component: Students had to self-identify themselves as Indigenous, be enrolled in any major offered by the institutions where the study took place, be in at least their sophomore year, and indicate their desire to participate in the interview. The researcher took steps took steps to protect the anonymity of participants. To do this, each participant received a name in Quichua language as a pseudonym. Twelve Indigenous students participated in this study; six males and six females. Interviewees ranged in age from 18 to 27 years old. The information for each individual is presented below.

Interviewee 1, Atuq (Sly) was a 21-year-old student who was born in a small Indigenous village called Rumicruz located in Calpi village. He shared that both his parents work in agriculture. He highlighted having learned Quichua from his grandmother. He was thankful to her for having taught him the language of his ancestors. At the time of the interview, Atuq still lived with his parents and siblings. He said that in his spare time and on weekends, he worked in a carpentry shop to contribute both to the family income and to his own daily expenses.

Interviewee 2, Inkillay (My Little Flower) was 19 years old. She came from Gerardo Morales village located in Cañar Province. Her father worked in agriculture 
while her mother was a homemaker. Inkillay was the fifth of six siblings. In addition to being a student, she was also working as a housekeeper on weekends. She shared that she did not wear her Indigenous attire anymore to avoid being discriminated by people.

Interviewee 3, Pumawari (Strong and Brave). Pumawari was 19 years old. He was born in Curiquinga village located in Columbe village. Both his parents worked in agriculture. This student was married and a father of a young baby. At the time of the interview, Pumawari was living in Curiquinga village. He said the population in his community has decreased significantly because people migrate to other countries, looking for better opportunities in life. However, he said he would stay there to support his village.

Interviewee 4, Yurak (White). This 18-year-old student was born in Baltar village located in Pungalá village. She was the fourth of ten siblings. She proudly mentioned being Puruhá. The Puruhás are indigenous people who have lived in central Ecuador for centuries. Puruhás mostly occupy much of the Chimborazo and Bolívar Provinces. Yurak's parents worked for farm owners. Although she had been living in the city for a while now, she admitted to missing her life in the countryside that she described as peaceful and content. Yurak has occasional jobs. She uses this money to cover her university expenses.

Interviewee 5, Mallki (Tree). This student was 19 years old at the time of the interview. He was born in Palmira located in Guamote parish. His father was a mason and his mother was a homemaker. He mentioned that most of his family including his siblings still lived in their village located in the countryside. He said that as a kid living in 
a small village, he was not aware of racial issues affecting Indigenous people. However, he started to experience prejudice as soon as he started his studies in the city.

Interviewee 6, Pacha (Earth). Pacha was born in an Indigenous village called Pompeya located in Licto parish. At the time of the interview, she was 23 years old. She said that before starting her college studies, she migrated to the capital to work. She decided to continue her studies in the hope that a college degree would provide her and her whole family with more legitimacy. She wanted to show others that Indigenous people are also able to succeed academically. Pacha had a job in her free time. Pacha shared that to her, working was a way of being grateful to her mom for all the effort she put to raise the family.

Interviewee 7, Jarawi (Poet). Jawari was 20 years old. This student was born Cajabamba. Cajabamba is a small town located in a fertile valley in Colta parish. Jarawi's mother worked for the city hall while his father worked in agriculture. He was the oldest sibling. He shared that to him, being the first essence implied being the role model to his younger brothers and sisters. As a role model, he was also a provider for the home since his father was unemployed.

Interviewee 8, Intiawki (Prince Sun). Intiawki was a 19-year-old student. He was born in Guamote parish. Both his parents worked in agriculture. He was the sixth of ten siblings. He said that out of his nine siblings, only five were attending school. He shared that despite his brothers and sisters were already living in different towns because of their jobs or private businesses, they always got together for special celebrations. He said that his family bonds were tight and provided him with the emotional support when he needed to cope with difficulties. 
Interviewee 9, Tamya (Rain). Tamya was 21 years old. Her parents worked in agriculture. She was born in Lorenzo de Telan village located in Guamote parish. Her family relocated to a bigger community when she was a child. The family had to leave their village because of the lack of pipe borne water supply. She was the second daughter of eight siblings. Only two of her siblings were attending school at the time of the interview. She explained that her siblings were not able to continue their studies because her parents were not able to afford school expenses. She felt fortunate to have the opportunity to study and indicated that she wanted to make the most of it.

Interviewee 10, Wayta (Flower). This student was born in Yuchi village. At the time of the interview she was 20 years old. Her parents worked in agriculture. She described her community as a peaceful place to live in. She shared that although she did not contribute financially to the family, she worked on the family farm on the weekends. She also shared that relocating to the city was a hard experience for her because, in her village, she engaged with Indigenous people like herself. She believed that speaking the same language and having the same traditions with the people in her Indigenous community made her feel more confident and happy.

Interviewee 11, Koya (Main Queen). Koya was a 27-year-old student. She was born in the Pulingui community located in San Andrés parish. She was married with two children. Her father was a bus driver and her mother was a homemaker. In her introduction, she said that this was her third attempt to obtain a college degree. She mentioned that the first time she withdrew form college was because she felt judged and discriminated by her teachers and peers. She mentioned feeling more empowered and confident of succeeding after having overcome past issues. 
Interviewee 12, Ninawillka (Holy Fire). Ninawillka was born in Gatazo village located in Cajabamba parish. He had turned 21 two days before the interview took place. He lost his mother at a very young age. His paternal grandmother raised him and his other siblings. Ninawillka was married and a father of a son. He shared that he had migrated to the capital when he was a child, but he went back to his village in his teenage years. He said that his experience in the capital had helped him navigate college life. $\mathrm{He}$ was currently working as a teacher in a small rural school.

\section{Experiencing Microaggressions}

Although Indigenous students' experiences with microaggressions were not associated with question four, it was my interest to include some of their most touching encounters with racial hostilities that emerged throughout the interview process. The rationale for considering these accounts is because they are the underlying stimuli that trigger students' responses, reactions, and subsequent adoption of coping mechanisms.

All 12 Indigenous students who participated in this study were able to describe encounters with racial microaggressions on issues linked to assumptions of inferiority, second-class citizenry and assumptions of criminality, microinvalidations, exoticization/assumptions of similarity, environmental microaggressions, and workplace and school microaggressions. Students' perceptions are presented with examples that provide evidence of their experiences while navigating college.

\section{Assumptions of Inferiority}

Indigenous students are commonly seen as socially, intellectually, and academically inferior. Participants described reasons for Indigenous people feeling 
ashamed of their race/ethnicity and the impact it has on their on their lives. Atuq's

account summarized reasons for being assigned as inferior,

I believe some Indigenous people are ashamed of who they are because Mestizos undervalue us for being Indigenous. Mestizos depreciate us for wearing a poncho, a hat, and an anaco $^{6}$. It seems to me like because of being Indigenous, we do not have voice, as if we were underclass people.

Jawari described how he perceived Indigenous people are deemed as inferior in terms of knowledge. He believed that Indigenous people are mainly judged because of their ethnicity and place of origin. Jawari stated the following,

Indigenous people are seen as less compared to others. It is as if this person comes from the countryside; therefore, this person does not know how to answer a question. Indigenous people are discriminated. Indigenous people are judged by their looks.

Intiawki provided another example of Indigenous people seen as intellectually

inferior. He mentioned that,

People believe I do not have enough knowledge because I am Indigenous, because I belong to other class. They believe such because I come from the countryside. They think I do not know enough.

Indigenous people are generally ascribed as less intelligent than Mestizo people

are. Yurak's experience depicted this claim. Yurak said that she did not usually wear her traditional attire on campus except on special occasions because she does not want other people question her capabilities. She recalled an incident where she decided to wear her Indigenous attire to present her project to the class. Yurak said,

It was clear that the teacher believed I was not capable enough to present in class when he saw me wearing my traditional attire. I do not think it was fair. In the presentation, I explained what my mother did as a traditional midwife. When I was finished with my talk, the teacher asked me "So, where are you from?" When I responded from Pungala (Indigenous village) he replied with surprise so are you Indigenous?! I said yes.

\footnotetext{
${ }^{6}$ Anaco is a type of skirt Indigenous women wear as part of their Indigenous traditional outfit.
} 
Koya also identified assumptions of inferiority related to Indigenous people's educational background. She considered that studying in the countryside is usually seen as a disadvantage. She explained,

They push us away, they make us feel inferior. We feel inferior with regards to our education. We usually think, if I had studied in the city, they would not treat me this way.

She continued to say that

They always say that we as Indigenous people, we do not know enough. They believe we do not have sufficient preparation for college. However, when they see we are able to use language correctly, they get very surprised.

Indigenous students perceived the way they use Spanish language is another reason for being targeted as inferior. Intiawki said,

Coming to study from the countryside to the city is difficult for us. When we start speaking and if we make a mistake, classmates start bullying us, humiliating us. Classmates start telling us that we do not have enough knowledge.

Wayta added another event where language became a source of racial microaggressions. She said, "They make fun of me because I am not able to speak clearly. They believe that because we are Indigenous, we are not able to succeed."

Koya recalled an event where a teacher admonished an Indigenous student in front of the class because of her mispronunciation. She remembered,

We as Indigenous people have difficulties to pronounce letters ' $\mathrm{O}$ ' and ' $\mathrm{U}$ '. Once a teacher told one of my classmates that he had to pronounce words accurately. She explained letter ' $\mathrm{O}$ ' is letter ' $\mathrm{O}$ ' and letter ' $\mathrm{U}$ ' is ' $\mathrm{U}$ '.

In her account, it appeared that pronunciation of Spanish language was a determinant of undervaluing Indigenous people's capacities. Koya complained that Indigenous students are constantly bullied because of language issues. 


\section{Second-Class Citizenry and Assumptions of Criminality}

Participants also reported being treated as second-class citizens and receiving differential treatment compared to people from groups holding more power. Pacha remembered an event that occurred to her as soon as she started college. She shared,

When I just started college, I used to wear my traditional attire. However, it was difficult for me to make friends. I recall the first school day. I was trying to find a spot to sit in the classroom. I took a seat next to this fancy classmate. She looked at me and then she started to call another girl asking her to take the seat. I felt very offended. It really hurt me.

Yurak shared a similar experience. She said, "People make me feel bad even with innocent suggestions such as saying, Hey! Go sit somewhere else (emphasis added)." Interactions with teachers were also described as occasions where Indigenous students feel as second-class citizens. Pacha mentioned,

In class, when I have a doubt, some teachers are open and kind with us, but others are not. They do not even let me get closer to them. I believe those teachers do not want us succeed because we are Indigenous. I do not think it is because we are bad people; it is just because we are Indigenous.

Perceptions of second-class citizenry go beyond the classroom and permeate to

other social spheres. Jawari remarked that colleges are largely judged by people based on the characteristics of the population they have. Jawari said,

If a college has a large number of Indigenous students, people say that university is not good. People say that is a college for Runas ${ }^{7}$ or Longos $^{8}$. On the contrary, if the population of a college is mainly White (Mestizo) and they are all well dressed, then people think the quality of education is better.

\footnotetext{
${ }^{7}$ Runa is the Quichua word for Human Being. However, it is used as a pejorative term to establish differences between White Mestizos and Indigenous people.

${ }^{8}$ Longo is pejorative term used to create a racial/class distinctions among White Mestizos and Indigenous. Longos are discriminated because of their dark skin color and looks.
} 
Interestingly, none of the interviewees reported having being labeled as a

dangerous person, criminal, or deviant person, based on their race or ethnicity. Also

worth noting it that none of the interviewees in this study referenced an experience where

Indigenous people were treated or deemed as criminals.

\section{Microinvalidations}

Indigenous students perceived being constantly microinvalidated about their names and

outfits. They also observed microinvalidations in the form of denying the existence of

racism. Yurak shared her experience in class,

When a teacher could not read my last name then all the students started to laugh.

Then the teacher asked me, Where are you from 've mamita.' I was so embarrassed; I never expected to live such an experience here in the city. That made me feel really bad.

This student was prolific in her descriptions of microinvalidations. In fact, among her stories, she remembered another event where she was wearing her poncho. She described the experience as,

Sometimes, when I am wearing my poncho, they stare at me and it is like if they ask themselves, Where are you from, what is wrong with you, why do you dress like that?

In the same vein, Yurak questioned people who deny the existence of racism. She shared,

I have always heard people saying 'I do not care who you are' but that is a lie. That is a lie for sure because when I wear my Indigenous attire or my poncho, people stare at me.

Yurak continued her account saying,

Although people say I do not care about your skin color, if you are Indigenous, they look at you as if - you know - but they say a lot behind your back. They do not dare to tell you what they think to your face. 
Another student perceived that racism is rampant despite people's disregarding its

existence. Pacha's example encapsulates the idea of being constantly microinvalidated.

She mentioned,

When I was in first semester, in one of our classes, we were taught about the Constitution. The articles stated that the Ecuadorian government declared there is not discrimination of any kind anymore and that we all deserve being treated the same. I believe this statement is just written on paper. In real life, this is not implemented.

Another student recalled feeling as being a foreigner or coming from a remote

place when she mentioned her village. Koya said,

At the beginning, every single teacher asked me what high school I had studied at. When I responded, they replied, "Where is that school located?" It was as if they had never known about my village or my school.

Reacting with surprise when an Indigenous student uses language correctly is also

a common occurrence associated with microinvalidations. Ninawillka shared,

When a Mestizo gives a public speech, nobody gets surprised of his good performance. Mestizos are believed to be well read, with multiple abilities, which is not necessarily true. However, when a person wearing a poncho and hat performs well, people get surprised. I believe that as Indigenous people, we try to demonstrate that we are as capable as others.

\section{Exoticization and Assumptions of Similarity}

This form of racial microaggressions also prevailed throughout the interviews.

Experiences where students were required to participate in events or get involved in activities for the sake of their ethnicity were common remarks made by Indigenous students. Yurak said,

Often times my classmates ask me how to say things in Quichua. They also want to know what we prepare with the guinea pig. I believe they ask questions because they are apparently interested in learning about my culture. However, I feel like they make fun of what I say because maybe they are unfamiliar with traditions or ancestral knowledge. 
Ninawillka expressed his frustration and sadness when his peers laughed at his ancestral language. Ninawillka said,

After a presentation I did in Quichua, some peers asked me for the translation of some words. When I translated the information for them, they just started to laugh. Ninawillka noted feeling at a loss when his classmates laughed at him.

People assign erroneous value to Indigenous attire and Quichua language. They see both as folkloric traits that generate curiosity for people. An instance where Indigenous attire and language were temporary convenient occurred to Inkillay. She mentioned,

When teachers assign group work, peers would object having an Indigenous student in the group. They would say, "She is Indigenous, so she does not have enough knowledge". However, when it comes to a special celebration like the Inti Raymi then would they say, "Let's take her in our group because she is Indigenous so we can ask her to wear her Indigenous attire and speak in Quichua."

Inkillay also recalled an incident with a teacher: "Once I came to campus wearing my Indigenous attire. Everyone looked at me very surprised. The teacher told me in front of my peers 'Congratulations for wearing your attire; you look beautiful in your pollera $^{9, .}$,

Atuq exemplified Indigenous people exoticization. He said, "Sometimes when there is a celebration such as the Inti Raymi, Mestizos would dance wearing Indigenous costumes. However, after the celebration is finished, they all go back to normal." Assumptions of similarity were associated with language use as depicted in Intiawki's account. He mentioned, "When presenting, I mispronounced a word. All my peers started to laugh, and I heard someone saying 'All Indigenous speak like that'..'

\footnotetext{
${ }^{9}$ Pollera, also known as Anaco, is the word used to name Indigenous womens' skirt that is part of their traditional attire.
} 


\section{Environmental Microaggressions}

Interviewees also assessed environmental microaggressions by referring to their perceptions of a campus environment where their culture and identity are made insignificant. Indigenous students' criteria revolved around the need for having more spaces that highlight their culture, so people get to know them better. Similarly, they called for opportunities to have open discussions about issues affecting them.

Inkillay shared, "I would like to see Indigenous culture represented on campus. I would like to see posters with messages on how the university supports our culture."

Atuq added more thoughts in this respect,

I personally believe that here at the university, there should be spaces to debate and discuss about our culture so we do not lose it and others understand it. Debate is necessary so people understand who we are and where we come from.

Tamia stated the importance of the representation of both ethnicities working together in harmony. She said,

I would like to see a picture of a Mestizo and an Indigenous student working together in the classroom. Images depicting both ethnicities together. I would like to see Indigenous people on my institution's web page. There are a few but I would like to see more.

Ninawillka insisted on the need of people having a better understanding of Indigenous culture to avoid discrimination. He said,

I would like to see my culture, my values represented on campus so Mestizo society gets to know us better. Discrimination is the result of ignoring who we are. If they knew who we are, they would treat us differently.

Yurak's opinion centered on diversification of activities and more presence of Indigenous culture on campus. She mentioned, "It would be useful if there were phrases in Quichua language around campus. That would help both teachers and students take 
into account our language." Another interesting remark from Yurak highlighted the limited spaces given to Indigenous culture on campus:

I have not seen activities other than the Inti Raymi. There are no open discussions about sensitive topics. They should bring an Indigenous person to give a speech. I believe activities like these are very scanty on campus.

Yurak instilled:

It would be a good idea to organize activities where our traditions and customs are put forward through plays maybe, not only the Inti Raymi. Through plays, we should be able to say what is actually happening in society.

\section{Workplace and School Microaggressions}

Accounts on workplace and school microaggressions were abundant throughout the interviews. Ninawillka said he has been constantly bullied because of his last name,

They make fun of my last name. They say 'Inca, Inca, vienes de los Incas' and then everybody starts laughing. I certainly believe this is an indirect way of being racist. They believe they are not offending me though, but they actually do.

Jarawi shared being bullied because of his Indigenous last name. He said, "I feel sad when people make fun of my last name. When this happens, I think to myself and say 'I hate my last name.' I would like to change it if I had the chance."

Being ignored or invalidating opinions were also common remarks. Mallki said,

Classmates simply do not value your effort. However, they do not tell what they think about you straight to your face. They would not tell you that you are unskillful. They would not do that. They would simply reject you. It is as...do not approach that person. That is what we feel. It is quite disappointing. Instead, they should try to help us. Similarly, teachers do not value our work. They simply ignore us.

Teachers also treat indigenous students differently, as Koya stated,

When I ask a favor to teachers, some of them change the way they treat me when they see I wear anaco. The way they treat Indigenous and Mestizas is different. They would tell Mestizas mija' or 'no worries at all.' However, they would not treat us the same. 
Jawari described demonstrations of rejection at school as "They reject you because of your looks, your outfits, or the way you speak. Indigenous people are excluded simply because of your looks." Another student also spoke about feeling rejection. Pumawari said, "Teachers believe that because students are Indigenous, they do not have enough knowledge. When Indigenous students answer questions in class, teachers would say 'No, no, no', that is not correct.” The cited examples of racial microaggressions briefly touched Indigenous students' experiences with hostilities. As I mentioned at the beginning of this section, I considered it necessary to include these accounts as a crucial element to enhance the understanding of how students respond to microaggressions in their college context. What lies ahead is an analysis of Indigenous students' responses and the coping mechanisms they adopt when facing racial hostilities. The analysis of these elements led to answering research question number four.

\section{Research Question Four. How do Indigenous Students Experience Racial Microaggressions while Pursuing a Degree in a Public Higher Education Institution on Issues of Responses to Microaggressions, and Mechanisms they use to Cope with \\ Microaggressions?}

\section{Responses to Microaggressions}

This section presents the findings for Indigenous students' responses to racial microaggressions. Several examples with abundant descriptions of internal battles, conflicts and feelings demonstrate participants' actions and feelings in reply to hostilities.

Immediately after microaggressions occur, they generate a response by the victim. Content analysis of the interview transcripts in both the initial and follow-up interview generated themes regarding students' responses that emerged from their lived experiences 
with microaggressions. Participants described a number of responses when being targets of a racial hostility. In general, these responses tended to be rather passive by either remaining silent or trying to explain to the perpetrator how they feel. In any case, students' responses were classified in two main themes: emotional and behavioral responses.

Emotional Responses. Emotional responses comprised a wide range of possibilities. The researcher categorized students' emotional responses in three subthemes: internal struggles, positive emotions, and negative emotions. A summary of themes and subthemes is for emotional responses is presented in Table 12.

\section{Table 12}

Summary of Themes and Subthemes for Emotional Responses to Racial Microaggressions

\begin{tabular}{ll}
\hline Theme & \multicolumn{1}{c}{ Subtheme } \\
\hline Emotional Responses & 1. Internal Struggles \\
& a. Making meaning of the event \\
& b. Ignoring or dismissing the event \\
& c. Setting Boundaries \\
& 2. Positive Emotions \\
& a. Enhancing Pride \\
& b. Building up resilience \\
& c. Demonstrating integrity \\
& 3. Negative Emotions \\
& a. Sadness \\
b. Anger/discomfort \\
c. Resignation/acceptance
\end{tabular}

Internal Struggles. Racial hostilities triggered internal struggles in students. Accounts showed three different types of responses that involved students in the process of internal dissonance. After the assault, students tried to make sense of the situation. 
They would ponder several questions in their attempt to rationalize the hostile event.

Their efforts are aimed to resist the attacks. Examples of questions are:

Why is this happening to me?

What did I do to provoke this animosity? or

Why is this person attacking me?

Another response that generated internal conflict was students trying to ignore the experience. Ignoring as a response to racial microaggressions resulted in two gains for students: protecting themselves and avoiding more conflict in the already racialized environment. A final response implied that students embark in a process of establishing their tolerance limits that will eventually determine if they adopted other type of responses.

Making meaning of the event. Immediately after Indigenous students perceived being harassed, they revealed going through a process of making sense of the hostile event. In their attempt to find an explanation, they start asking themselves a series of questions. For instance, Koya's response was trying to find if something in her behavior caused the hostility. She shared,

When I notice an adverse behavior against me, I start asking myself, 'What did I do? What happened? Why did this person tell me this? I remain in doubt. I would like to know the truth. I would like the person to tell me what I did wrong.

In this example, it was clear that in a sense, Koya blamed herself for having caused the offender's behavior.

Other responses showing victims undergoing internal struggles included trying to find the reasons for being harassed. Intiawki shared, "When I receive an offense, I think that I would like to know why the person did that to me." Mallki's questions were 
oriented to knowing if the perpetrator was conscious of their hostile behavior. He wondered, "Why doesn't this person realize what he is saying?" Jawari's internal struggles aimed toward assigning value to the hostility. He said, "When people make fun of me, I ask myself 'Why should this impact me?' If I figure out it was just a joke, then I decide it will not impact me."

Asking questions regarding the hostile event went beyond finding the reasons that triggered it. Questions also revolved around the common racial background people share. Koya said that when someone mistreats her, she asks herself,

It is like your skin is a bit lighter than mine, or your last name is-I don't knowBenitez, so you have the right to mistreat me? I do not know. I have always asked myself why if we all have Indigenous ancestry, so why do we mistreat each other?

From the answers cited above, it was clear that students' responses were replete with internal struggles. Additionally, these responses implied students investing extra energy in processing their emotions. Although students said that in a sense they are accustomed to living in uncomfortable situations because of their race, they still experience internal battles as a consequence of microaggressions.

Ignoring or dismissing the event. Ignoring the event or at least trying to do so was the most common response endorsed by participants. Ignoring seemed to give Indigenous students the serenity they needed to continue with their activities despite being the subject of systematic disrespectful actions against them. For instance, Inkillay said, "I try not to give the event much importance because that would make me feel even worse. I had better said, 'I will ignore the person'." Pumawari shared a similar opinion:

One has to let it go because sometimes the person who mistreats does not know about the other person's intelligence or capacity. One has to listen and let it go. One should not take it too personal. Just let it go. 
However, Pumawari also responded to microaggressions by engaging in a sort of internal selection process of taking the positive and discarding the negative comments. Pumawari stated,

I have gone through a lot of things in my life. If they say good things about me, I embrace those words. If they say bad things I just ignore. I listen and evade whatever they say. Those comments do not stay with me. I just ignore.

Jawari also manifested that ignoring was the best approach to respond to microaggressions. Though he confessed being tempted to react verbally or physically he said that by ignoring, he protects himself from further events. Jawari said,

I have realized that when I ignore people who make fun of me, it is like if they little by little start forgetting to tease me. That is why I prefer to remain silent. I just listen to them while they laugh at me. At some point, they get tired of me. Once they get tired of me, they do not harass me anymore.

Avoidant responses to an offense are also an option for Indigenous students. This is so because they do not want to get involved in situations that are worse than the hostility itself. Pacha's testimony reflects this assertion. Pacha said,

When I notice a negative attitude or behavior against me, I pretend I did not notice it. I pretend I did not hear, like if nothing happened. Life is like that. If we complain or do something, it is even worse. Ignoring is what we have to do.

Pumawari shared the same viewpoint. He shared,

I believe ignoring is a better approach. It is better to let it roll off your back. It is also avoiding conflict. Like being slapped on the right cheek and turn the other. It is better to ignore. It is better to avoid getting into trouble.

Setting boundaries: Another response that involved an internal struggle by

students was intuitively establishing a line that defined their tolerance to a racial hostility. Jarawi said, "I tolerate harassment to a certain extent. If the hostility goes beyond my tolerance level, then, I respond." However, during the interview, Jawari was not able to 
describe the specific line of his tolerance level. Trying to establish a tolerance limit was also an internal struggle for Yurak. She said, "Sometimes it is like they take you to your limit, and then you react and say, 'Hey, what is wrong with you? Why do you mistreat me? " Intiawki was able to identify the borderline between ignoring an aggressive behavior against him and responding to it. He said, "I have a limit. When people reach my limit, I respond. Sometimes people are very overt and rude when saying things and that is my limit."

Positive Emotions. Interviewees also recalled responses that helped them affirm their identity as Indigenous people. In some cases, racial hostilities generated a resilient response where students were able to recover quickly. Their responses also demonstrated toughness toward pursuing their goals and succeeding for themselves and their families. Furthermore, students mentioned that their self-esteem was enhanced after being microaggressed.

Pride. As members of a group that has been systematically marginalized, answers provided by interviewees demonstrated microaggressions made them even more proud of their ethnicity. Their responses were in terms of upholding their background despite facing racial hostilities. Pumawari remembered an occasion where he perceived having been harassed. His response was,

Well, on my side, it did not impact me because whatever they say stays with them. I am Indigenous and I value my culture. It might affect other Indigenous people. They may even grow in resentment and shame when people discriminate them. In my case, it does not touch me. I am who I am, and I will fight for my family and me.

Pumawari's response of ethnic validation was, "When they call me Indio, I respond, "Yes, I am Indio." When they call me Indigenous I respond, "Yes, I am 
Indigenous." Pumawari's statement is remarkable because it encompassed the honor and dignity he gave to his ethnicity because, despite the negative connotation of the word Indio, he still felt proud of being Indigenous. Similarly, Yurak's response to microaggressions was one of pride of her ethnicity. She shared, "When someone wants to put me down, I try to not to give much importance to those negative comments. I am absolutely proud of my race. I am certainly proud of my race.” Finally, Tamya said, "When they tell me I am Indigenous, I respond, "Of course I am." I know for sure where I come from and I do not feel embarrassed about that."

Resilience. This subtheme emerged from Indigenous students' accounts of having the capacity to recover from the hostilities they face in their daily college activities. Responses were a demonstration of toughness, resistance, and tenacity. Resilience was evident in Koya's answer,

When these things happen to me, I have never cried. I remember once I was about to cry but I did not. I felt that courage and bravery inside me. I told to myself, 'you can do this'. I also thought if they are capable to do things, I am capable as well. I always look for my inner strength. These thoughts help me calm down and face situations.

Atuq voiced his resilient response as,

Obviously, you feel bad but at the same time you try to forget the event and move forward. As a student, I do not care about others' comments. I just want to be better than those who despise me.

Jawari's response to racial hostilities was also one of consolidating his strength.

Jawari said,

When someone puts me down, I start saying to myself 'why should this person's comment make me feel bad if I know who I am. When we know our capabilities, then people's comments do not have an impact on us. 
Students also associated their responses with their motivation to succeed in college. Mallki said that his encounters with hostilities had sometimes pushed him to abandon his studies. However, his response has been to resist despite the circumstances. He shared, "I have thought of giving up college several times. I have continued despite how people make me feel. I have decided to continue studying because I am a person of courage." Mallki's resilience resided in the fact that his family has expectations on him. He described, "Right now, my goal is to succeed. My family has put a lot of hope in me. I am the first one in college. They want me to graduate. I will graduate because of them.” Resilient responses were associated with students' aspiration to thrive in their studies. Inkillay stated, "I will continue to study even though they mistreat me. No matter what happens, I will succeed. I will succeed because that is my goal, that is what I want and that is what my parents want." Likewise, Pumawari's responses were of resilience and attempts to improve. He stated, "My experience in college has been quite difficult with peers who tell me things that hurt. Despite those words, I have tried to succeed. I have tried to progress in the sense that I have done a lot of effort to overcome my own limitations."

Demonstrating integrity. Indigenous students appealed to their moral and ethical convictions to respond properly to adverse hostile circumstances. In one of the accounts, an interviewee described how his ethnicity and the way he was raised dictated his response. Ninawillka highlighted his values as an Indigenous man. He said, "If I decide to respond to a person's negative comment, I try to have a conversation with the person. Because of my values as Indigenous man, I do not react aggressively." Ninawillka further explained, "I respond peacefully because I am a person of values. We have been taught to 
ignore." Indigenous students demonstrated their integrity by putting others first. Intiawki said, "I sometimes respond verbally but not in a bad way. I do it kindly because I do not want to offend others." Similarly, Mallki reported, "I do not respond because I do not like hurting anybody." He reiterated his statement twice: "I do not like hurting anybody." Pacha said that she usually evaluates the situation before responding. Her response demonstrated her view of the different set of values Indigenous people have compared to Mestizos. Pacha reflected,

If I start questioning them or arguing, they will blame me even more. As Indigenous, it is hard for us to insult others because we were not educated in that manner. However, it is easy for them to offend others. They are used to insulting.

Finally, although Jawari exhibited integrity in the way he responded, he did not deny the impact microaggressions had on him. Jawari shared, "I respond calmly because I am a peaceful person. Of course, I feel bad about it but then I just do not give much importance."

Negative Emotions. Participants voiced their responses as a replete of negative emotions such as sadness, discomfort, shame, and pessimistic acceptance to their faith. One common expression to most participants when describing their negative emotions was "I feel bad." Participants at the request of the researcher explained what this expression meant.

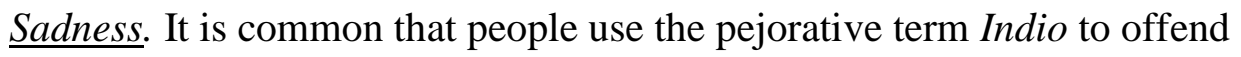
Indigenous people. This is Ninawillka's response when he heard people calling him Indio: "When they call us Indios, I feel bad." When asked to describe what feeling bad meant to him, he said, "I feel isolated, offended, hurt, uncomfortable and sad." In his description, he used intense terms to transmit his emotions when being disrespected by 
the word Indio. Inkillay also used the expression "I feel bad" several times when describing her response to an insult. She explained what "feeling bad" was to her, Well, to me it is feeling sad. Sometimes you think and say 'I want to leave this place. I do not want to come back here anymore. I want to go to another place. I want to look for other possibilities.'

Intiawki described his feeling of sadness in response to encounters with racial hostilities. He shared,

When I made a pronunciation mistake in class, they laughed at me. That made me feel very sad. I asked myself, 'why do they discriminate me? Why don't they tell me the correct word instead of laughing at me?'

Finally, Jawari indicated, "I feel sad when people make fun of my last name. I start thinking that I hate my last name, I would like to change it.”

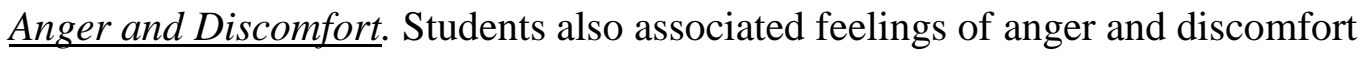
as responses to racial hostilities. Pumawari's account was remarkable. He explained that on campus, his peers called him by his nickname Longo. As stated before in this account of experiences with microaggressions, Longo is an appellative term used to describe people with Indigenous heritage spotted by their darker skin, use of Spanish language and appearance. The term is pejorative indicating rejection and hostility from White mestizos to Indigenous people. He shared he did not feel offended when people called him Longo because he was used to it. He said that Longo actually reflected his peers' appreciation to him. However, when asked if he responded differently if people called his daughter Longa, he said, "If someone called my little girl Longa, I would get annoyed. I would respond angrily for sure. I would try to defend her. I would try to protect my little girl." It was clear that although he said he did not get angry when people called him by his 
nickname, his response would change if this term was used for his closest family. His answer demonstrated that Pumawari was aware of the harm the word Longo has on people.

Ninawilla described his feelings of anger and discomfort when being mistreated. He said, "It is feeling bad, insecure, and angry_-very angry." Pacha responded with discomfort. She said, "When they mistreat me, that makes me feel bad. For them that is kind of normal, but it really hurts me. It makes me feel bad." Tamya said that at some point in her program of studies, students mistreated her. She shared,

I used to feel lonely because it was only me as Indigenous student. Mestizos were the majority, so they befriended with each other. I felt uncomfortable and scared. I was scared of being discriminated. I was scared that my opinion was not valued.

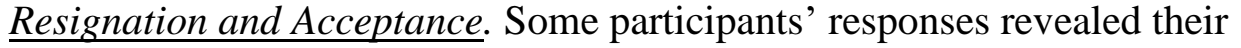
acceptance of the undesirable but inevitable aggressive behaviors of people around them. Jawari gave an overwhelming answer that reflected the resignation he has created as a response to microaggressions. Jawari shared, "Eventually, it did not hurt anymore. I got numb. I no longer heard what they said, so it did not hurt." Intiawki went through a long process before reaching his resignation line. His response to hostilities was "At some point in my life, I had negative thoughts. It does not happen anymore. I just do not give importance to what people say about me." A response with a taste of giving up was also present in Inquillay's answer. She said, "I take it as it is. I keep myself calm. I do not say anything”. Finally, Ninawillka's response was one that combined resignation and resilience. Ninawillka reported, "One is used to be discriminated. One is used to be ignored, but one has to demonstrate that one is a person of value." 
Behavioral Responses. Aside from emotional responses, a few Indigenous students stated they went further to respond with actions. These responses mainly consisted of verbal interactions to try to explain their viewpoints, enhance harmony in the environment, and maintain their balance. Behavioral responses included voiced responses where victims sought to educate the offender, demonstrate empathy, and ask for the respect they deserve. Table 13 summarizes the subthemes for behavioral responses.

\section{Table 13}

Summary of Themes and Subthemes Behavioral Responses to Racial Microaggressions

\begin{tabular}{ll}
\hline Theme & \multicolumn{1}{c}{ Subtheme } \\
\hline Behavioral Responses & 1. Voicing feelings \\
& a. Educating the offender \\
& b. Demonstrating empathy \\
& c. Asking for respect \\
\hline
\end{tabular}

Voicing Feelings. Indigenous students said that they mainly responded to microaggressions verbally. In their responses, it was evident that Indigenous students did not intend to hurt or embarrass the person or give the offense back. They aimed to convey their true feelings, be honest, and reconcile the environment.

Educating the offender. Three students acknowledged they responded to racial microaggressions verbally. They said they did not respond angrily but gently. A subtle intention to educate their offenders emerged from their accounts. Yurak said that a couple of times she has tried to talk with the peer who mistreated her. She said she would appeal to her classmates' reflection of their improper behavior. She reported saying, "Why do you treat me like this? Don't you realize that you have Indigenous ancestry as well? Do 
you think because you have a nice last name you are superior to me?" Ninawillka provided a similar answer. He said, "When someone is hostile with me, first, I ignore it. I listen passively, and then I try to explain them how uncomfortable I feel with their comments." Koya explained she hardly ever responded orally. However, when she did, she tried to establish a dialogue with the peer. She said, "When I respond, I start asking questions like why you behaved like that. I explain the importance of respecting people no matter who they are. I believe that is a good option."

Demonstrating Empathy. Indigenous students' responses also leaned toward demonstrating empathy for the people who offended them. Inkillay said, "Sometimes when I respond, I tell the person "Please do not mistreat me because you would not like any of your relatives or your children to suffer what I am suffering." Koya's response was twofold. She first asked herself the motive that triggered the person's behavior and then she tried to rationalize the person's feelings. She shared,

I ask myself why is this person behaving this way? Then, I assume that maybe the person is going through difficult times. Sometimes I ask 'Are you okay?' At the same time, I reconsider the situation and think if this person is experiencing a hard moment. It is not my fault.

$\underline{\text { Asking for respect. }}$. Albeit scarce, students' verbal responses pleaded for respect to their ethnicity. Pumawari's response described a feeling of anger when people called him Indio. He disputed the difference between being called Indio and Indigena. Pumawari said,

Sometimes I react a bit angrily when they call me Indio. I say, 'you cannot call me Indio. You can call me Indígena. Indio is as if you are lazy, you do not work, and you do not have knowledge. Indio is an offense. It is like if the person is a criminal. I believe it is an offense. 
Another participant shared an experience where he raised his voice to support a peer who was being bullied. Atuq described his response as "feeling good because I was able to express what I felt that moment. I just sought for some more respect for us as Indigenous."

\section{Mechanisms Students use to Cope with Microaggressions}

This final section of the qualitative analysis presents findings related to students' mechanisms to cope with racial microaggressions. Interviews revealed that students engage in the adoption of actions that help them mediate the stress created by hostilities.

Two main themes for coping strategies emerged from students' accounts. The first was identified as seeking interpersonal support and the second one was opting for stress relief activities. Students seeking interpersonal support would search for parental and family support, as well as friends' support. Stress relief activities involved numerous activities ranging from practicing sports to reading the Bible. Table 14 presents a summary of themes and subthemes for coping mechanisms.

\section{Table 14}

Summary of Themes and Subthemes for Coping Mechanisms

\begin{tabular}{cl}
\hline Theme & \multicolumn{1}{c}{ Subtheme } \\
\hline Interpersonal Support & 1. Parental/immediate family support \\
& 2. Friends'support \\
Stress Relief Activities & 1. Easing up anxiety \\
\hline
\end{tabular}

Interpersonal Support. Indigenous participants discussed the importance of having their parents and close family members support as generators of the necessary support they need to cope with racial hostilities. 
Parental and Immediate Family Support. Indigenous students' accounts suggested they found the strength and support they needed in their immediate family. Strong bonds among family members were also clear in students' stories. Participants' accounts gave parents a crucial role in helping them cope with oblivious demonstrations of racism. Mallki shared,

I talk about these issues with my parents because I trust them. My parents tell me I can achieve my goals if I fight for what I want. They say I should do not let people think I am weak. I should show people my strength. They keep telling me that I just have to continue with my studies. That is how they support me.

Koya cited examples of her parents' advice that give her the strength she needs to continue her studies and cope with racial hostilities. She said,

I talk about these issues with my parents because I trust them. My parents tell me I can achieve my goals if I fight for what I want. They say I should do not let people think I am weak.

Koya denied needing support other than that of her parents. She emphasized, "I

have never thought of going to a psychologist. To me, talking to my parents is enough to get back to normal. I do not need anybody other than my parents.”

Atuq wanted to find answers that explain motives for Indigenous people being mistreated so it is easier for him to cope with them. He said,

I have not talked about these issues with people other than my parents or close relatives. Sometimes I ask my parents, 'Why does this happen to us? 'They say, 'Look, things are not as bad as before...We are not as powerless as we used to be...Indigenous people have also succeeded.'

Finally, Tamya established the role of parents and classmates when talking about racial concerns. She acknowledged, "I talk about these issues with my parents but not with my classmates. I trust my parents more. I really feel their support. I also talk to my siblings. Classmates are just friends here at the university. They are friends but not to talk 
about these issues." Tamya believed that her parents had earned her trust because of their unconditional support and advice when she had faced hostilities.

In looking for minimizing the impact of racial microaggressions, the Indigenous students reported that they also appeal to their siblings. Inquillay trusts her siblings since they share the same experiences as she has. She gave reasons for not trusting other people. Inquillay said, "I talk to my siblings because I live with them. I just trust my siblings. I can talk with my siblings about what happens to me and what happens to them. We talk about the way we are treated by people." She continued to explain her doubts about trusting classmates or teachers. Inquillay stated,

I do not talk with my classmates or with my teachers. I just do not trust them. Sometimes when I tell something to a classmate, then this person tells it to others. I do not like it when rumors are spread. If I trust someone, that someone has to be discreet. I do not talk with teachers either. When I tell them something, they spread the word.

Friends' Support. Only one student reported trusting friends as a mechanism to cope with racial hostilities. However, he mentioned it could not be any friend but an Indigenous one. Intiawki said,

I look for support in my Indigenous peers. They have lived the same experiences as I have. I believe we Indigenous people have more life experience than Mestizos do. I have looked for support in people who have lived what I have lived.

Intiawki continued to provide more reasons for trusting his Indigenous peers. $\mathrm{He}$ shared, "My Indigenous peers can tell me what to do. My Indigenous peers always recommend me not to give up."

Stress Relief Activities. Analysis of data suggested that Indigenous students used a variety of active strategies to deal with racism besides talking to their family and friends. Participants shared a mix of activities that help them forget the hostile events, 
reduce stress and support their spiritual and mental health. These activities were for the most part beneficial for students in terms of promoting their growth and positive feelings.

Easing up Anxiety. Microaggressions boost stress levels in victims. Consciously or subconsciously, Indigenous students involve themselves in actions to build up their tolerance to stress. The following examples illustrate how students ease up anxiety. Inquillay stated, "I release my stress by watching movies. Sometimes I listen to motivational reflections, then I think to myself I will be fine." Mallki shared, "Sometimes I watch videos on YouTube. I watch videos of people who were poor but succeeded despite adversity. It really motivates me."

Appealing to spiritual actions that contribute to ease students' suffering were options seen as assets for students. Pacha claimed,

I am a Christian. Therefore, when something negative happens to me, I read the Bible. The Bible says that, in God's eyes, we are all equal. God does not see color or race. In the Bible I find the peace I need. I read and reflect. To me, what God says is more important than what people say.

Finally, a wide range of alternative activities to release stress was considered as an option for students. Intiawki stated, "When people offend me, I prefer going out with a friend to play, go for a walk, or have some food.” Jawari mentioned, “Oftentimes, I go out with my friends. We go to play soccer or to do something that I enjoy doing. I try to forget the incident by engaging in different activities." Similarly, Yurak said, "Sometimes I try to let it out by writing. This is my only way to release stress." Pumawari mentioned, "I listen to or play music when I want to release my stress. I sometimes go running. It really helps clear my mind. While running, I feel free.” 


\section{CHAPTER FIVE}

\section{DISCUSSION}

This chapter elaborates on results that emerged in Chapter Four. The chapter starts with a discussion of major findings as they relate to the existing literature on microaggressions. This chapter also discusses the limitations of the study, recommendations and implications, and suggestions for further research. This chapter closes with a conclusion of the research.

Existing research recognizes that as brief and commonplace daily indignities, racial microaggressions take a toll on people's mental and physical health (Gomez et al., 2011; Pierce et al., 1978; Reid, 2017; Sue et al., 2007). A search of the literature also reveals that college campuses are not exempted from issues associated to racism. This study examined racial microaggressions of Indigenous students in a public higher education institution. This study aimed to answer the following research questions:

1. What are the most/least common types of racial microaggressions that Indigenous students experience while pursuing a degree in a public higher education institution?

2. What is the association between microaggressions Indigenous students' selfesteem?

3. What is the association between microaggressions Indigenous students' selfefficacy?

4. How do Indigenous students experience racial microaggressions while pursuing a degree in a public higher education institution on issues of responses to microaggressions and mechanisms they use to cope with microaggressions? 


\section{Occurrence of Racial Microaggressions}

The first research question in this study explored the most/least common types of racial microaggressions that Indigenous students experience while pursuing a degree in a public higher education institution. Pierce (1988) contended that implicit racial derogatory messages are present in all kind of settings such as workplaces, campuses, schools, and public spaces. Previous studies concur in that college campuses are places where underrepresented people face a myriad of hardships that affect their success, academic performance, and their mental and physical well-being because of racial motives (Grahame, 2004; McCabe, 2009; Samuel \& Park, 2015; Vaccaro, 2010). Despite its prevalence, the literature states that, unfortunately, racism is so deep-rooted in colleges that, at times, it is difficult to recognize (Chesler \& Crowfoot, 1989; Harwood et al., 2012; McCabe, 2009; Solórzano et al., 2000; Solórzano et al., 2009).

A major finding in this study suggests the prevalence of racial microaggressions affecting Indigenous students at their college campus. Results indicate that students encounter all the types of racial microaggressions contained in the REMS. This study uncovered that assumptions of inferiority and microinvalidations were the most common hostilities perceived by participants, followed by workplace and school microaggressions, exoticization/assumptions of similarity, and environmental microaggressions, respectively. The second class and assumptions component of criminality was perceived as the least common hostile occurrence students confronted.

As mentioned in the literature review, Sue et al (2007) and Nadal (2011) suggested that racial and ethnic underrepresented groups encounter certain forms of microaggressions more commonly than others do. The authors also suggested that 
experiences with racial hostilities differ among groups. Forrest and Jenson (2015) used the REMS to analyze Asian, Latino/Hispanic, Black, and White undergraduate students' perceptions of microaggressions. All groups reported differences in the type of microaggressions they perceived as the most and least common. In reference to the frequency that students reported the racial occurrences, of these three groups, Black students showed the highest encounters with microaggressions, followed by Latino/Hispanic, Asian, and White students. Aligned with the notion that experiences of microaggressions vary among groups, this current research determined that Indigenous students' identification of the most and least common microaggressions is dissimilar to the groups studied by Forrest and Jenson. Likewise, the frequency of microaggressive incidents in this study suggests variations among groups, Indigenous students being those who reported the highest occurrence compared with the groups analyzed by Forrest and Jenson.

\section{Microaggressions and Self-esteem}

A major component of well-being is self-esteem. Feeling confident and satisfied with ourselves is essential because such influences beliefs we create about our skills, abilities, and social relationships (Sedikides \& Gress, 2003). Although self-esteem mostly develops as an individual and internal process, it is also associated with the view that others have of the person (Baumeister \& Leary, 1995; Yeung \& Martin, 2003). The second research question in this study sought to establish the relationship between racial microaggressions and Indigenous students' self-esteem.

Correlation results show that assumptions of inferiority, second class and assumptions of criminality and workplace/school microaggressions have a significant 
negative association with students' self-esteem. These results indicate that an increase of these three types of racial hostilities may result in the decrease of individuals' reported self-esteem. Examples of the types of microaggressions that may be linked to a lessening of students' self-esteem are:

- Questioning Indigenous students’ intelligence,

- Assigning them low educational level because they did not have the opportunity to study in urban schools,

- Relating poverty with low academic performance,

- Sending disapproval messages because of ethnicity.

In accordance with the present results, previous studies have stated associations between experiences of racial microaggressions and self-esteem that can be detrimental for student's well-being (Harris-Britt et al., 2007; Nadal et al., 2014; Tawa et al., 2012; Thai et al., 2017).

Findings of the current study revealed negative association between microinvalidations and exoticization/assumptions of similarity; however, the association was not significant. Additionally, environmental microaggressions reported a positive non-significant correlation with self-esteem. Although these results differ from some published studies (Harris-Britt et al., 2007; Umaña \& Updegraff, 2006), this discrepancy could be attributed to Indigenous students' misunderstanding of some of the items in these specific components of the REMS or the inability of the instrument to capture all the nuances associated to racial microaggressions (McCullagh, 2007). Another possible explanation for these results might be that students are unaware of hidden racialized messages around them; or even worse, they may assume these hostilities as everyday 
occurrences they have to live with for the sake of their ethnicity. However, it is necessary to highlight that differences in significance levels for each component of the REMS in this study are congruent with Nadal's (2014) results. Nadal's results showed that selfesteem was negatively correlated with only two of the six REMS components namely second class citizen and assumptions of criminality. Nadal suggested that it is likely there are other mediating variables that may contribute to the relationship between the two constructs. Overall, on the basis of the results of this study, it can be argued that microaggressions are part of the lives of Indigenous students and that these hostilities have an impact on their self-esteem. However, since these hostilities go unnoticed by students, they may cause even more profound and long lasting effects on students' selfesteem. More research on the specific impact of racial microaggressions on Indigenous student's self-esteem is needed to properly address this issue.

\section{Microaggressions and Self-efficacy}

Self-efficacy is an important component of the self-system that plays a role in how we behave and address different situations in life (Bandura, 1977). Believing in our potential to do things is crucial because it determines how we feel about ourselves as well as our capabilities to achieve goals. According to Bandura (1995), the environment where people develop their activities acts as a catalyst to affirm or nullify self-efficacy. As mentioned in the literature review, self-efficacy is not only crucial for well-being, motivation, and personal attainment but for student's academic and social adjustment (Linnenbrink \& Pintrich, 2003; Nichols, 2007; Williams \& Rhodes, 2014; Zimmerman, 1995). The purpose of the third question in this study was to examine the associations between microaggressions and Indigenous students' self-efficacy. 
Results showed that the correlation between racial microaggressions and students' self-efficacy was negative and significant for five of the six components of the racial microaggressions survey: assumptions of inferiority, second class/assumptions of criminality, microinvalidations, exoticization/assumptions of similarity, and workplace/school microaggressions. These negative correlations indicate that an increase of these five types of racial hostilities results in the potential decrease of students' reported self-efficacy. It is worth noting that workplace and school microaggressions showed the highest negative correlation. This finding may suggest that hostile behaviors such as being unfriendly with students because of their ethnicity, overlooking their opinion, ignoring them, neglecting their opinions, assuming their work as inferior, and being treated differently than their peers contribute to undermining Indigenous students' self-efficacy.

An interesting result emerging from the correlation analysis between the six REMS components and self-efficacy inventory is that environmental microaggressions was the only component of the REMS that reported a positive relationship. There are a number of possible explanations for this result. A potential reason may be that as it was stated for self-esteem results, participants may have had trouble understanding the message implied in some of the questions in the REMS. However, another possible explanation for this is that students may not give much importance to seeing people of their ethnicity portrayed positively in movies, magazines, television or occupying major positions in big companies. It also seems possible that students are not aware of the value of being represented as an ethnic group in major spheres. A noteworthy remark of this discussion is the identification of similar behaviors in the results obtained for self-esteem 
and self-efficacy. Results in this study revealed that the positive association between environmental microaggressions and self-efficacy equated with the one obtained with self-esteem. The observed similarity in the association between both variables may be partly explained by the fact that, apparently, daily direct interpersonal hostilities have more impact on students' lives than those indirect occurrences happening at a macrolevel. This assertion might be valid because, from the nine categories of microaggressions identified by Sue et al. (2007), environmental microaggressions seem to be the most difficult to identify and remedy.

Another similar behavior in the results was that self-efficacy and self-esteem reported the highest negative significant correlation values for workplace and school microaggressions. In other words, the more incidence of workplace and school hostilities, the more detrimental the consequences for students' self-esteem and self-efficacy. This finding illustrates how unwelcoming behaviors - for example, pathologizing students’ personal and academic abilities, giving them different treatment, bullying, or ignoring them — can take a toll on students' psychological well-being. Results for the associations between self-esteem, self-efficacy, and microaggressions in this study suggest that closer attention should be payed to school racial hostilities since they may translate into students' experiencing negative satisfaction in the school environment and difficulty in establishing bonds to their institutions (Bailey 2016; Dogan, 2015; Forrest-Bank \& Jenson, 2015; Laurent, 2016; Linnenbrink \& Pintrich, 2003; Nadal et al., 2014; Warner, 2019).

A final similarity between self-esteem and self-efficacy results deals with the average scores reported by Indigenous students for these constructs. Results indicate that 
the overall scores for self-esteem and self-efficacy were higher than expected. These results may be explained by the fact that Indigenous students may have developed specific approaches that help them tackle racial discrimination. It also seems plausible that their upbringing prepares them to shield against hostilities because their parents usually warn them of the hostile environment they may encounter once they leave their Indigenous communities. These rather surprising results may also be related to Indigenous students developing mechanisms of resilience that help them resist and recover easily from the difficulties they encounter in racialized environments.

In the view of these results, further work is required to address additional questions that emerged throughout this study. For instance, there is room to determine the impact of racial microaggressions on students' academic self-efficacy. A need for more literature that examines variables such as students' motivation, achievement, and students' academic and social adjustment to college is necessary for a better understanding of the impact of microaggressions on Indigenous students. It is my belief that a thorough analysis of issues regarding microaggressions may result in institutions adopting adequate policies that protect and boost underrepresented students' self-esteem and self-efficacy so they are able to navigate college more effortlessly.

The first part of this analysis discussed the findings from the quantitative component in this study. These quantitative findings provided insights to the understanding of racial microaggressions in aspects such as occurrence, most and least prevalent types of hostilities students encounter, and the associations between microaggressions and self-esteem and self-efficacy in a numerical fashion. However, this first approximation to racial microaggressions needed live examples that illustrate the 
experiences of Indigenous students with the phenomenon under study. This mixed method study employed a sequential transformative strategy to exemplify racial microaggressions. Creswell (2008) recommends this approach for studies exploring issues related to inequality, injustice or oppression as in this study. What follows is the narrative of Indigenous students' lived experiences with microaggressions in their institution. Their accounts revealed the details of the hidden and intimate feelings of participants while dealing with racial hostilities. These accounts uncover the oppressive, discriminatory, and inequitable expression of microaggressions.

\section{Microaggressions Experiences}

Despite the fact that the initial goal of question four was to look at responses and coping mechanisms students adopt to fight hostilities, this section starts with a review of students' experiences with racial microaggressions. Analyzing participants' accounts is essential to have a clear picture of the phenomenon examined in this study.

In the qualitative component, the researcher interviewed 12 Indigenous undergraduate students who provided their ideas, thoughts, and feelings regarding their experience with microaggressions, their responses when being targeted by hostilities, and the coping mechanisms they adopt to battle aggressions. Students' lived accounts supported the presence of racial microaggressions in powerful quotes that evidenced their oppression. This study demonstrated that Indigenous participants experience all kind of microaggressions on campus. This finding is congruent with the previous literature in that college campuses can be uninviting and threatening places for underrepresented students where institutions have not been able to properly address issues of oppression, marginalization, and inequity (Solórzano et al., 2000; Solórzano et al., 2009) 
Students concurred in that they are seen as inferior in several aspects.

Interviewees said they are deemed as academically and intellectually inferior for the sake of their ethnicity and place of origin. Indigenous students are constantly marginalized when peers and teachers question the quality of education they receive in the countryside and their scholastic abilities. Another source for Indigenous students to be considered as inferior is language. Difficulties students have in pronouncing Spanish puts them at a disadvantage and makes them targets of ridicule in the classroom.

Indigenous students' opinions coincided in that teachers and peers address them as second-class citizens since they receive differential treatment compared to their Mestizo counterparts. Interviewees mentioned having difficulties in making friends on campus, as well as receiving obvious verbal and behavioral signals of people feeling uncomfortable when dealing with them. Students acknowledged that the negative differential treatment they received did not have a reason other than their ethnicity. However, students' opinions indicated that, although they are treated as second-class citizens, they are not seen as criminals or dangerous. This assertion may be explained by the fact that Indigenous people are still stereotyped as peaceful and submissive. Interviewees reported suffering from constant microinvalidations. Two main types of microinvalidations were identified in their responses. One was related to Indigenous students' feeling as "different" especially when they wear their traditional attire on campus. They referred to people staring at them or making apparent "innocent" comments like "You look beautiful in your Indigenous outfit." Although these comments may seem racially innocuous and well-meaning, there is a hidden message of segregation and disadvantage for recipients (Sue, et al., 2008). 
The other type of microinvalidations mentioned by students was colorblindness. Students criticized the naïve position of peers and teachers in negating the blatant racial stratification and the privileges of Mestizos over Indigenous students on campus. Students believe that the claim of equity to all declared in the constitution is far from being implemented to its fullest. According to Mazzocco (2017), because of its apparent neutrality, colorblindness is a detrimental ideology that hides the impact of race on underrepresented groups and precludes addressing the issues associated to racism.

Respondents described exoticization of Indigenous culture as a sort of diversity of convenience. Interviewees perceived that they were welcomed as Indigenous only in festivals and celebrations where the contribution of their language, music, dances, and costumes were valued. However, the amiable behavior of Mestizo peers vanished after festivals ended. According to participants, this apparent inclusiveness depends on specific circumstances rather than a real appreciation of Indigenous people and culture. In regard to assumptions of similarity, students indicated that Mestizos believe incorrectly that all Indigenous students have the same difficulties in the pronunciation and use of Spanish. This explains why Mestizos are surprised when they hear an Indigenous person using the language in a proper manner. In relation to environmental microaggressions, Indigenous students described them as the scarcity of representation of Indigenous culture on campus. Interviewees agreed in that the school environment does not contribute toward making their culture visible. They expressed the lack of events that support them enhancing their values, discussing issues, and making people more knowledgeable about their culture. Students insisted in that the discrimination they face is the result of people's ignorance and the misunderstanding of Indigenous cultural philosophy. 
In terms of school microaggressions, participants described these types of hostilities as common events on campus. School microaggressions were in the form of being bullied because of their looks or last name that to some extent make students repudiate their Indigenous origin. Students also concurred that people ignore their efforts toward succeeding in their studies despite the adverse circumstances they face in their daily lives. Students provided examples of multiple difficulties they experience that go unnoticed by peers, teachers, and authorities. Students mentioned that financial and family issues push them to looking for jobs even if doing so only yields minimum wage. Combining work and studies is challenging for students because they have to juggle the time they allocate to attending to class and completing their assignments. Additionally, Indigenous students face the hassles of having to commute every day, sometimes for more than two hours from their parishes to the city or rent a room that results in their experiencing feelings of loneliness for being far from their families. School microaggressions also included being ignored in their opinions and invisible in their needs that result in students' profound disappointment and frustration. Students referred to scarcity of spaces where everybody is involved in critical conversations regarding racism. They mentioned that the curriculum does not support their need to enhance their culture.

In light of students' perceptions of microaggressions occurrences on campus, I believe that these racial hostilities operate as mechanisms that result in the oppression of Indigenous students. In this particular case, Indigenous students suffer from what Young (2004) calls structural oppression which refers to common, every day, vast, and profound inequities oppressed groups suffer as a result of dominant groups unconscious 
assumptions that incorrectly stereotype, minimize, and relegate oppressed groups because of their race, class, gender, sexuality, and/or other particular affiliation.

The analysis of manifestations of racial microaggressions; associations between microaggressions, self-efficacy, and self-esteem; and the experiences of students with racial hostilities led to the identification of race as a structure of oppression for Indigenous students in four out of five faces of oppression proposed in Young's (2004) model.

\section{Marginalization}

Although marginalization is associated with material deprivation and lack of financial autonomy, it is also related to issues of marginality in the form of uselessness, boredom, and lack of self-respect. (Young, 2004). Indigenous students' stories were prolific in describing acts that relegate them to a lower standing in their institutions. However, they prefer to remain silent in the margins because, as they mentioned several times, it is safer for them although doing so precludes their rights as human beings. Silence was exemplified by one of the interviewee's who said, "I pretend I did not notice it. I pretend I did not hear, like if nothing happened. Life is like that. If we complain or do something, it is even worse. Ignoring is what we have to do."

Students are also constantly minimized in their needs and to an extent, obliged to remain silent under the premise of preserving harmony in their school environment. Remaining silent is definitely the main cause of their marginalization because as Young (2004) states, "it also involves the deprivation of cultural, practical and institutionalized conditions for exercising capacities in a context of recognition and interaction" (p. 51). 


\section{Powerlessness}

Results from this study are quite revealing of Indigenous students' lack of power by virtue of their "underclass" ethnicity. Powerlessness is manifested in their scanty participation in decision making that affects their lives and in actions they take that yield space and authority to other groups (Young, 2004). As a group with little or no power, Indigenous students are controlled by others and therefore subjected to take orders. Another manifestation of powerlessness is Indigenous students' silence. In their accounts, students mentioned that they preferred to remain silent when a hostility occurred to them. Students' silence was also accompanied by a feeling of resignation to their fate as in this narrative "I take it as it is. I do not say anything. One is used to be discriminated, one is used to be ignored." Unfortunately, by remaining silent, they lose the capacity to raise their voice to ask for justice.

Additionally, interviews with participants made evident that some of them remain unaware of the multiple manifestations of racism, especially those that are more elusive such as jokes and nicknames. Although apparently innocuous, jokes and nicknames convey detrimental messages Indigenous students are sometimes not aware of. An example of this lack of awareness was the student who said that his peers called him Longo as a sign of acceptance to him. Silence and lack of awareness are unequivocal signs of students losing spaces of power in their environment. Freire (2000) states that powerlessness is dangerous especially when people are not aware of their own position as oppressed or when they are scared of talking about their oppression because they are afraid of subsequent retaliations. When people are not aware of their fate and even worse, when they are scared of raising their voice, they are condemned to everlasting ignominy. 


\section{Cultural Imperialism}

This study suggests that the clearest manifestation of oppression of Indigenous students is cultural imperialism. Participants complained of being culturally deceived because their cultural manifestations receive little or no importance. Students contemplate that their culture draws attention only in the context of festivities or religious parades. However, after the celebrations people resume their everyday roles where cultural separation between Indigenous and Mestizos is manifested. Indigenous students' testimonies evidenced that there is a clear dominant group whose cultural expressions receive more relevance and is assumed as the paragon to be emulated. Some examples provided by students in the interviews were the following: adopting Spanish language, modifying their pronunciation to minimize Quichua language traces, changing outfits, embracing behaviors similar to those of their Mestizo peers, and even adjusting their eating habits.

The adoption of the culture of the majority is consistent with Martinez and de la Torre's (2010) perspective of how educational systems contribute to eradicating Indigenous culture. The authors explain that Indigenous students are compelled towards mestizaje to be accepted and gain more legitimacy in their institutions. Young (2004) explains that cultural imperialism means "[experiencing] how the dominant meanings of a society render the particular perspective of one' group invisible at the same time as they stereotype one's group and mark it out as the Other." Young indicates that the dominant group assumes the role as the normal or standard to imitate by other groups if they want to fit. In other words, Mestizo culture permeates the identity of Indigenous people but the reverse process does not occur. 


\section{Violence}

Although subtle, microaggressions are attacks against oppressed people who live under constant threaten that erodes their physical and mental health. These unprovoked assaults are consciously or unconsciously oriented to damage, humiliate, and destroy the person in the form of harassment, intimidation, or ridicule (Young, 2004). Indigenous students are victims of racial hostilities for the sole reason of their race. For instance, students receive remarks that humiliate them because of their Indigenous last names as the account provided by one student who complained being harassed because of his family name "I certainly believe this is an indirect way of being racist." According to Young, violence is a phenomenon of social injustice that prevents people from their full realization as human beings both for themselves and for the society. Violence is a social practice where everyone knows that happens but anybody takes steps to stop it.

\section{Responses to Microaggressions and Coping Mechanisms}

Indigenous students' responses to microaggressions revealed two main categories identified: emotional and behavioral. Emotional responses included internal struggles, positive emotions and negative emotions. Internal struggles implied students' asking themselves a series of questions to make sense of the incident; most of these questions attempted to figure out what they as victims did to trigger the hostility. Additional evidence of students going through internal struggles was their effort to ignore or dismiss the hostile event because of two reasons, protecting themselves from further hostilities and avoiding more conflict in the already racialized environment. Internal struggles also involved students' trying to establish their tolerance limits to a racial hostility as in the following example: "I tolerate harassment to a certain extent. If the hostility goes beyond 
my tolerance level, then, I respond." However, in many cases, students were unable to define their tolerance limit. Defining their tolerance level was crucial because it would determine the occurrence of a verbal or even a physical reaction.

Although racial hostilities convey unfriendly messages toward Indigenous people, their responses are not necessarily negative. Results suggested that racial hostilities generated positive responses in Indigenous students. The majority of respondents agreed that racial microaggressions generated a sense of pride and affirmation of Indigenous identity in them. They took the negative behaviors or comments in their environment as reasons for them to be prouder of their Indigenous background, their places of origin, and their families. They even took the word Indio not as an offense but as a powerful reason to continue in their search for success. In this study, Indigenous students demonstrated that a solid ethnic identity both mitigated the negative effects of discrimination and made them less vulnerable to the impact of racism. It is clear that throughout their experience of oppression, these students develop strong attachments and a sense of belonging to their ethnic group that shields them against hostilities. This finding is crucial since research indicates that ethnic identity operates as a protective factor that helps people contest detrimental behavioral and social experiences augmenting their self-esteem, decreasing depressive symptoms, and improving academic adjustment (Neblett et al., 2012; Phinney, 1996; Shawn \& Neblett, 2016, Stein et al., 2014).

Responses to racial indignities also increased Indigenous students' resilience. They demonstrated ability to transform racialized occurrences in opportunities to re-adapt themselves in the face of adversity. Indigenous students concurred in that they had to fight harder than their peers in demonstrating to everyone that they were also capable of 
achieving high goals. Indigenous students found their families as the main source for their resilience. Their family's moral and spiritual support gave students the strength to find a purpose and go beyond themselves. Families were the motive to persist and accomplish their academic goals. This assertion was supported with opinions such as: "Right now, my goal is to succeed. My family has put a lot of hope in me. I am the first one in college. They want me to graduate. I will graduate because of them," and "I will continue to study even though they mistreat me. No matter what happens, I will succeed. I will succeed because that is my goal, that is what I want and that is what my parents want."

Another positive response that students annotated in their accounts was the importance of demonstrating integrity despite offenses against them. Indigenous students highlighted that their parents have taught them to take offenses and respond with the respect with tolerance and respect. Students emphasized that their parents educated them in a way that regardless of the offenses they received, they would always try to avoid hurting people back. Indigenous students' response was one of holding on their cultural values, philosophies, and ideas upon which they exist. The following testimony summarizes Indigenous students' integrity: “As Indigenous, it is hard for us to insult others because we were not educated in that manner. However, it is easy for them to offend others. They are used to insulting."

Although the majority of Indigenous students affirmed responding positively to racial hostilities, some interviewees agreed in that microaggressions provoked negative emotions as responses in them. Sadness was the most common response provided by participants. Indigenous students developed feelings of isolation, discomfort, and defeat 
as in "I feel isolated, offended, hurt, uncomfortable and sad." Sadness reflected students" frustration for not having a clue to explain the constant discrimination they suffered because of their ethnicity. A second response to racial hostilities was anger. Students did not deny that they felt offended when people mistreated them especially with the use of the pejorative word Longo/a or Indio/a. Respondents explained the anger racial microaggressions generated in their lives using terms such as frustration, annoyance and irritation. Students' anger evidenced intense emotional states that eroded their spirit as in this short but resentful statement "It is feeling bad, insecure, and angry—very angry." However, no interviewee mentioned providing a verbal or physical aggressive reaction to an offender. A third negative response given by Indigenous students after being microaggressed was identified as resignation. Participants shared that at some point they developed a kind of numbness to racial hostilities that ultimately left them defenseless. The following narrative illustrates this claim "Eventually, it did not hurt anymore. I got numb. I no longer heard what they said, so it did not hurt.” They mentioned that eventually they got used to be harassed and discriminated. This is particularly dangerous because they become unable to feel, reflect or react to racialized occurrences.

Most Indigenous students reacted to racial microaggressions emotionally;

however, some of them described responding behaviorally. Behavioral responses yielded only one subtheme identified as voicing feelings. Although a few students admitted to responding to racial hostilities verbally, their attitude was one of looking for reconciliation by educating the offender, demonstrating empathy or asking for respect. According to interviewees, they tried to educate their offenders in the sense that mutual consideration was paramount to maintain a good atmosphere. Indigenous students would 
also try to make offenders realize that mistreating people for the sake of ethnicity is not acceptable because to an extent, everybody shares the same Indigenous descent. Additionally, students responded by demonstrating empathy with their offenders. Interviewees wanted to figure out if offenders had suffered discrimination or harassment that made them act aggressively. This response let Indigenous students resolve the conflict and move forward. Students would also raise their voice to ask for respect to their persona and culture, especially when people call them Indios. Students claimed that being called Indio is disrespectful because of the implications associated with this word.

Overall, students' responses to microaggressions were in the form of passive behaviors where victims decided on either remaining silent or ignoring the hostility. On one hand, Indigenous students believed that adopting a passive response was the best approach to avoid more conflict and to stay safe in their environment. On the other hand, when students verbalized a response, they did not want to contest or argue with the offenders but rather make them understand their behavior was misleading.

Concerning coping mechanisms Indigenous students adopt to deal with the effects racial microaggressions, respondents' answers were classified as: seeking interpersonal support and involving in activities that helped them relieve their stress.

Interpersonal support was found to be the most common coping mechanism Indigenous students reported. Students did not engage themselves in intentionally seeking a mentor or a psychologist to help them overcome their emotions. On the contrary, tackling racial hostilities for Indigenous students was a natural spontaneous action that interviewees engaged in by looking for support in their immediate circle. Students found the sustain they needed in their family. For the most part, students believed that only their 
parents or siblings were able to understand how they felt. Indigenous students assumed that since their parents and siblings have gone through similar adverse conflicts, they were able to provide the best advice for resolving challenging situations. Participants stated that their beloved ones encouraged them to ignore hostilities and move forward with their studies as in the lines below,

I talk about these issues with my parents because I trust them. My parents tell me I can achieve my goals if I fight for what I want. They say I should do not let people think I am weak. I should show people my strength. They keep telling me that I just have to continue with my studies. That is how they support me.

Students also described talking to their parents or siblings as a reciprocal support. Participants said they were not able to develop trust in people other than their family. A few interviewees mentioned they relied on the support of friends to cope with microaggressions. However, they considered that counting on Indigenous friends was more beneficial than counting on Mestizo friends since their Indigenous counterparts equipped to understand their feelings and frustrations. Indigenous students' opinion in this respect was depicted as:

I look for support in my Indigenous peers. They have lived the same experiences as I have. I believe we Indigenous people have more life experience than Mestizos do. I have looked for support in people who have lived what I have lived.

Besides interpersonal mechanisms, Indigenous students also engaged in other types of stress relief activities to tackle microaggressions such as going out with friends in their free time, doing exercise, writing, reading, and listening to music. Interviewees highlighted that listening to or reading motivational stories of people who have succeeded despite adversities inspires them to advance with their personal and educational pursuits. Stress relief activities were aimed to stimulate reflection and forgiveness. 


\section{Recommendations}

This study found that Indigenous students endure all kinds of microaggressions on campus. Interviews revealed that students lack awareness of ways to identify racial hostilities, respond, and deal with them. This lack of understanding makes the impact of microaggressions more oppressive and detrimental for victims. In that racial hostilities are harmful, the literature assigns institutions a pivotal role in truly addressing racial issues to support racially and ethnically diverse students succeed and critically navigate college (Espinosa et al., 2019; Gudeman, 2000; Helm, 2013; Marin, 2000; Maruyama \& Moreno, 2000; Milem \&Hakuta, 2000). Helm (2013) recommends higher education institutions first acknowledge the existence of subtle demonstrations of racism on campus to further design appropriate strategies that provide underrepresented students with inclusive and safe environments. Nadal et al., (2014) also emphasize the importance of creating consciousness regarding racial awareness and urge institutions to guide vulnerable students in identifying racial hostilities and building shields to protect themselves against racial occurrences. They also call for institutions to take agency in the creation of programs that promote harmony and inclusion to all students. Similarly, Umana and Updegraff (2007) support the idea of institutions demonstrating their interest in supporting ethnically and diverse students by performing appropriate assessments of the racialized dynamics present in educational settings that lead to creating mechanisms that prevent them.

Given the importance of creating awareness regarding racial issues, Thai et al., (2017) offered a suitable strategy for sensitizing the college community. They recommended that institutions use socialization messages that enlighten all students on 
the transmission of cultural traditions of battling oppression. The authors maintained that these messages might help underrepresented students navigate racialized environments with dignity and pride of their culture. Additionally, the authors suggested the importance of racial socialization as a protective factor of self-esteem. Similarly, Blackmon et al (2015) acknowledge the importance of racial-socialization in institutions and encourage them to adopt measures that foster cross-racial relationships and intercultural understanding that improve students' coping, resilience and adjustment to college.

Throughout this study, Indigenous students' opinions revealed the presence of subtle forms of prejudice, discrimination, and biases in the classroom, not only from peers but also from teachers. Teachers either ignore racial hostilities, treat Indigenous students differently, or address Indigenous culture incorrectly. Respondents also complain about teachers not giving enough spaces to engage in difficult discussions about race, social justice, and approaches to reducing the negative effects of racism. In their accounts, Indigenous students assign their teachers a paramount role in both educating other students regarding racism and supporting them in fighting against this phenomenon. Indigenous students demand that teachers be more knowledgeable about Indigenous people's ways of knowing, doing, and learning. In this respect, Mallinger et al. (2016) suggest that an adequate intervention from faculty is crucial to alleviating the stress and discomfort created by racial microaggressions in the classroom. Including relevant content in the curriculum, selecting appropriate materials that provide with multiple perspectives, and creating inclusive classroom communities are some of the recommendations provided by the authors for teachers toward helping students tackle racial microaggressions. 
Indigenous students complained of cultural invisibility on campus and the lack of spaces that help them navigate college critically. Students demanded a meaningful representation of Indigenous culture in every sphere of their institution. Participants agreed in that the institution maintains superficial policies and that there is fictitious pluralism where diversity of convenience is the norm rather than the exception. Evidence of Indigenous students' apparent representation is the fact that the university participates with ethnic foods and festivals such as the Inti Raymi while failing to provide equal access and opportunity to students. Participants provided examples of actions their institution can take to give Indigenous culture more relevance on campus. Students wanted their language to be present on the institutional website especially in the mission and vision statements. Students also demanded Indigenous representation in the university council so that authorities hear their voices. They believed that having Indigenous representatives would result in the adoption of policies that benefit them as a group, especially with respect to aspects such as scholarships and the support they need to succeed.

Students also suggested that the institution should thrive for having more instructors with Indigenous background. Respondents believed that having Indigenous instructors on campus would motivate them to pursue their goals, contribute to improving their education, and benefit from a culturally responsive pedagogy. Finally, Indigenous students require that their institutions support them in the creation of counter spaces outside the classroom where they can meet, befriend, and share their experiences. These spaces might help students build a sense reflective community where they can support each other, battle isolation, discuss issues affecting them and create group resilience. 
In addition to creating awareness of racial issues, fostering relevant discussions, and including Indigenous culture as part of the college's life, this study calls for higher education institutions adopt of policies that improve the recruitment, retention, and graduation rates of Indigenous students. In relation to recruitment, institutions should create programs that support students making a smooth transition from high school to college that may include guidance for career choice, appropriate assistance for adjustment to campus life, and the discussion of sensitive topics that foster students' awareness regarding difficulties they may encounter in college. Institutions can also work on the design of retention strategies that create spaces where students receive academic and social support as well as activities that create bonds between the institution and students. Lastly, institutions should work on improving Indigenous students' graduation rates by adopting a more culturally sensitive instruction that empowers students in their realization and values the potential they bring to college. Institutions should commit to serve all students; especially those who have been systematically underrepresented by adopting a comprehensive approach that helps everybody navigate college safely and successfully.

\section{Limitations}

This study has discussed important implications regarding racial microaggressions affecting Indigenous undergraduate students. There are a few salient limitations to this study. Although this research used quantitative and qualitative methods to have a more thorough understanding of the phenomenon, the following considerations should be taken regarding its results and findings. Participants enrolled in one public higher education institution were selected in this study; since the sample was drawn from only one 
institution, generalizations to other colleges in Ecuador should be carefully considered. It is also advisable to consider the specific social, political, and environmental characteristics of the population in this study. Respondents represented the individualities of the target group that may differ with other Indigenous populations in Ecuador and other countries. Additionally, this study calls for recruiting larger populations of Indigenous students attending other college campuses all over the country to obtain a more accurate perception of this phenomenon.

Another limitation in this study was the use of the Racial and Ethnic Microaggressions Scale (REMS). This scale has been used in numerous contexts; however, the REMS may not have been the most accurate scale for assessing racial microaggressions for the population in this study. Nadal (2011) acknowledges this limitation, suggesting "It may be difficult to measure microaggressions for people of various racial and ethnic groups because of their racial and phenotypic appearances may yield different types of microaggressive incidents" (p. 478). Furthermore, the author advocates for a more detailed analysis that examines alternative factor models by modifying items to the original REMS scale or creating innovative measuring tools that better capture experiences of people in other settings. For example, Indigenous students' voices in this study revealed acculturation and isolation patterns that may be included in the design of a microaggressions scale tailored for this population.

A final limitation to this study is associated with the data collection methods utilized. The sole use of interviewing technique restricted the information to the participants' ability to recall their lived experiences truthfully. Additionally, this recall may not be accurate enough to capture details such as circumstances, people's 
interactions, and behaviors that may be neglected consciously or subconscious by participants in their accounts. The use of interviews and observation would be appropriate to a deeper and rich understanding of racial microaggressions.

\section{Implications for Further Research}

This study is pioneer in examining racial microaggressions in underrepresented college populations in Ecuador. Its findings have opened up a promising future for looking at this phenomenon in more detail. Interviews conducted in this current study revealed that Indigenous students experience racial microaggressions that to some extent are different from those contained in the REMS. These differences attend to the particular sociocultural context of Ecuadorian society. In the view of these variations, we recommend further research in the definition of the components and items in the original REMS scale that better identify the unique nuances that microaggressions take in Indigenous populations. Further inquiry on microaggressions may lead to the development of such an innovative microaggressions instrument that responds to the specific attributes of Ecuadorian Indigenous students.

Although most interviewees said they were proud of their indigenous background, some female participants mentioned having to leave aside their Indigenous attire by adopting a more Mestiza appearance that consists of wearing jeans and jackets. Female Indigenous students say that they adopt this look to gain more acceptance and avoid being discriminated on campus. Some of these students abandoned their traditional costumes permanently while others said they would wear as Mestizas on campus and as Indigenous in their communities. Further research should be undertaken to understand the process of acculturation Indigenous students undergo when switching to Mestizaje. It 
would be useful to determine the impact this change has on their identity and the relationships they establish with close relatives, friends, and members of their Indigenous communities once they have started their process of acculturation.

\section{Conclusion}

This study sought to give voice to Indigenous students with regard to racial microaggressions. The lens adopted throughout the study helped to determine that the oppression of Indigenous has not been eradicated despite the propaganda of equity, justice and inclusion in higher education institutions.

This study has been one of the first attempts in examining racial microaggressions in a public higher education Ecuador. The findings indicate that Indigenous students experience all type of racial microaggressions in their campus and that these hostilities are systemic and systematic. Although subtle, racial hostilities take a toll in the lives of students in multiple ways. Despite the numerous examples provided by students in their accounts and the prevalence of racial microaggressions in all spheres, the phenomenon is overlooked and even rationalized. I believe that the first step in tackling this issue is acknowledging that there is subtle racism affecting the lives of Indigenous students. The next step is to create awareness of the occurrence of racial microaggressions to both victims and perpetrators. A final stage is the adoption of institutional measures that contribute to create positive, successful and inclusive environments to all students. 


\section{REFERENCES}

Abouserie, R. (1995). Self-esteem and achievement motivation as determinants of students' approaches to studying. Studies in higher education, 20(1), 19-26. https://doi.org/10.1080/03075079512331381770

Albo, J., Núñez, J., Navarro, J., \& Grijalvo, F. (2007). The Rosenberg Self-Esteem Scale: translation and validation in university students. The Spanish Journal of Psychology, 10(2), 458-467.

Allen, Q. (2010). Racial microaggressions: The schooling experiences of Black middleclass males in Arizona's secondary schools. Journal of African American Males in Education, 1(2), 125-143.

Almeida, J. (1996) El racismo en el Ecuador: un problema de identidad. (Unpublished Masters Thesis). Flacso. Quito, Ecuador.

Ari, D., Cheser, L., Asghar, R. (2002). Introduction to research in education.Wadsworth Thomson Learning.

Arshad, M., Imran, S. S., \& Mahmood, K. (2015). Self-esteem and academic performance among university students. Journal of Education and Practice, 6(1), 156-162.

Avalos, S. (2012). The Indigenous quest for power-sharing in post-colonial Ecuador. Intercultural Communication Studies, 21(1), 140-153.

Bagley, C., Bolitho, F., \& Bertrand, L. (1997). Norms and construct validity of the Rosenberg Self-Esteem Scale in Canadian high school populations: implications for counseling. Canadian Journal of Counselling, 31(1), 82-93.

Bailey, K. (2016). Racism within the Canadian university: Indigenous students' experiences. Ethnic and Racial Studies, 39(7), 1261-1279.

Bandura, A. (1977). Self-efficacy: Toward a unifying theory of behavioral change. Psychological Review, 84(2), 191-215.

Bandura, A. (1982). Self-efficacy mechanism in human agency. American Psychologist, $37,122-147.4$

Bandura, A. (1994). Self-efficacy. In V. Ramachandran Encyclopedia of human behavior (Vol. 4, pp. 71-81). New York: Academic Press.

Bandura, A. (1995). Self-efficacy in changing societies. Cambridge University Press. 
Baumeister, R., \& Leary, M. (1995). The need to belong: desire for interpersonal attachments as a fundamental human motivation. Psychological Bulletin, 117(3), 497-529.

Beck, S., Mijeski, K., \& Stark.M. (2011). Que es racismo? Awareness of racism and discrimination in Ecuador. Latin American Research Review, 46(1), 102-125.

Bishop, G. F., Fischer, B. (1995). Secret ballots and self-reports in an exit-poll experiment. Public Opinion Quarterly (56), 568-588.

Bogdan, R., \& Knopp, S. (2016). Qualitative research for education: an introduction to theories and methods (5th ed.). Pearson Education.

World Bank Group. (2015). The World Bank Group Report: Indigenous Latin America in the twenty-first century: the first decade.

http://documents1.worldbank.org/curated/en/145891467991974540/pdf/Indigenou s-Latin-America-in-the-twenty-first-century-the-first-decade.pdf

Basco, L., \& Han, S. (2016). Self-esteem, motivation, and anxiety of Korean university students. Journal of Language Teaching and Research, 7(6), 1069-1078.

Baumeister, R., Leary, M. The need to belong: Desire for interpersonal attachments as a fundamental human motivation. Psychological Bulletin, 117(3), 494-529.

Beverly, D. (1992). Talking about race, learning about racism: The application of racial identity development theory in the classroom. Harvard Educational Review, 62(1). https://equity.ucla.edu/wp-content/uploads/2017/01/Tatum-Talking-AboutRace.pdf.

Blascovich, J., \& Tomaka, J. (1993). Measures of self-esteem. In J. Robinson, P. Shaver, \& L. Wrightsman (Eds), Measures of personality and social psychological attitudes (3rd ed., pp. 115-160). Ann Arbor: Institute for Social Research.

Bourdieu, P., \& Passeron, J.-C. (1977). Reproduction in education: Society and Culture. SAGE.

Bowen, G. (2008). Naturalistic inquiry and the saturation concept: A research note. Qualitative Research, 8(1), 137-152.

Branden, N. (1969). The psychology of Self-esteem: A revolutionary approach to selfunderstanding that launched a new era in modern psychology. Jossey-Bass.

Brenlla, M., Aranguren, M., Florencia, M., \& Vásquez, N. (2010). Adaptación para Buenos Aires de la escala de autoeficacia general. Interdisciplinaria, 27(1), 7794. http://www.scielo.org.ar/pdf/interd/v27n1/v27n1a06.pdf. 
Brown, D. (2004). Fighting racism in the twenty-first century. Washington and Lee Law Review,61(4), 1485-1499.

Brown, J., Dutton, K., \& Cook, K. (2001). From the top down: Self-esteem and selfevaluation. Cognition and Emotion, (15), 615-631.

https://psycnet.apa.org/record/2001-11208-004

Burdsey, D. (2011). That joke isn't funny anymore: Racial microaggressions, color-blind ideology and the mitigation of racism in English men's first-class cricket. Sociology of Sport Journal, 28(3), 261-283.

Burgoon, J. (1981). Learning theory approaches to persuasion. Human Communication Research, 7(2), 161-179.

Canel, D., \& Yohani, S. (2019). Indigenous Canadian university students' experiences of microaggressions. International Journal of Advanced Counselling (41), 41-60.

Chavez, E. (2008). Ser Indígena en la educación superior ¿Desventajas reales o asignadas? Revista de la Educación Superior, 37(4), 31-55.

Chesler. M., Crowfoot, J. (1989). Racism in higher education: An organizational analysis. PCMA Working Paper Series. The program Conflict Management Alternatives at the Univesity of Michigan.

Clark, R., Anderson, N., V., C., \& Williams, D. (1999). Racism as a stressor for African Americans: a biopsychosocial model. American Psychology, (54), 805-816.

Constantine, M. (2007). Racial microaggressions against African American clients in cross-racial counseling relationships. Journal of Counseling Psychology, 54 (1), $1-16$.

Creamer, E. (2018). An introduction to fully integrated mixed methods research. Thousand Oaks. Sage.

Creswell, J. (2008). Educational Research. Planning, conducting, and evaluating quantitative and qualitative research. Pearson.

Creswell, J. (2009). Research design: Qualitative, quantitative, and mixed methods approaches ( $3^{\text {rd }}$ Ed.). Sage.

Creswell, J., \& Plano-Clark, V. (2011). Designing and conducting mixed methods research. Sage. 
Crispen, C. (2017). The relationship between the campus climate and underrepresented students' experiences on campus and the influences on fit, self-efficacy, and performance: A qualitative study. (Publication No. 10820638). [Doctoral Dissertation, University of Southern California].ProQuest Dissertations \& Theses Global.

De-Cuir-Gunby, J., \& Gunby, N. (2016). Racial microaggressions in the workplace: A critical race analysis of the experience of African American Educators. Urban Education, 51(4), 390-314.

De-Cuir-Gunby, J., Johnson, O., Womble, C., McCoy, W., White, A. (2019). African American professionals in higher education: Experiencing and coping with racial microaggressions. Race Ethnicity and Education. (1)17. https://www.tandfonline.com/doi/full/10.1080/13613324.2019.1579706

Delgado, R., \& Stefanic, J. (2001). Critical Race Theory. New York University Press.

Dogan, U. (2015). Student engagement, academic self-efficacy, and academic motivation as predictors of academic performance. Anthropologist, 20(3), 553-561.

Doyle, S. (2007). Member checking with older women: A framework for negotiating meaning. Health Care for Women International, 28(10), 888-908.

Drywater-Whitekiller. (2017). We belong to the land: native Americans experiencing and coping with racial microaggressions. The Canadian Journal of Native Studies, XXXVII(1), 153-174.

Erel, D. (2000). The concept of self-efficacy and self-efficacy-performance relationship. Ankara University SBF (55)4. http://10.1501/SBFder_0000001924

Etikan I., Abubakar, S., Alkassim, S. (2016). Comparison of Convenience Sampling and Purposive Sampling. American Journal of Theoretical and Applied Statistics. (5)1, pp. 1-4. http://article.sciencepublishinggroup.com/html/10.11648.j.ajtas.20160501.11.html

Fabara, E. (2013). Estado del arte de la formación docente en el Ecuador. Cuadernos del contrato social por la educación. Contrato Social por la Educación Ecuador. http://contratosocialecuador.org/images/publicaciones/cuadermos/8.pdf

Fairclough, Sh. (2005). Self-esteem and academic achievement: A comparative study of African American students in a traditional public school and a magnet school. [Doctoral Dissertation, The University of South Florida. t: http://scholarcommons.usf.edu/etd 
Fisher, A., \& Shaw, C. (1999). African Americans' mental health and perceptions of racist discrimination: the moderating effects of racial socialization experiences and self-esteem. Journal of Counseling Psychology (46), 395-407.

Franklin, J., Smith.W., \& Hung, M. (2014). Racial battle fatigue for Latina/o students: a quantitative perspective. Journal of Hispanic Higher Education, 13(4), 303-322.

Freire, P. (2000). Pedagogy of the oppressed. (1st Ed.). Continuum International Publishing Group.

Friedlaender, C. (2018). On microaggressions: cumulative harm and individual responsibility. Journal of Feminist Philosophy, 33(1), 5-21.

Gachet, I., Grijalva, D., Ponce, P., \& Rodriguez, D. (2017). Vertical and horizontal inequality in Ecuador: the lack of sustainability. Social Indicators Research. (1)40. https://doi.org/10.1007/s11205-017-1810-2

Galletta, A. (2013). Mastering the semistructured interview and beyond: From research design to analysis and publication. New York University Press.

Golding, J., Aneshensel, C., \& Potts, M. (1991). Stress exposure among Mexican Americans and non-Hispanic whites. Journal of Community Psychology, 19(1), 37-59.

Gomez, M., Freitag, M., Khurshid, A., Johnson, A. (2011). Microaggressions in graduate students' lives: How they are encountered and their consequences. Teaching and Teacher Education. (27), 1189-1199.

Gonzalez, K. (2002). Campus culture and the experiences of Chicano students in a predominantly white university. Urban Education, 37(2), 193-218.

Grahame, K. (2004). Contesting diversity in the academy: Resistance to women of color teaching race, class, and gender. Race, Gender \& Class, 11(3), 1-19.

Guba, E. (1990). The paradigm dialog. Sage.

Guba, E. (1981). Criteria for assessing the trustworthiness of naturalistic inquiries. Educational Communication and Technology Journal, 29(2), 75-91.

Harper, M., \& Cole, P. (2012). Member checking: Can benefits be gained similar to group therapy? The qualitative report, 17(2), 1-8.

Harris-Britt, A., Valrie, C., Kurtz-Costes, B., \& Rowley, S. (2007). Perceived racial discrimination and self-esteem in Africa American youth: Racial socialization as a protective factor. Journal of Research on Adolescence, (17), 669-682. 
Helm, C. A. (2013). Relationship between racial microaggressions and psychological wellbeing of African American college students. (Publication No. 3596324) [Doctoral Dissertation, University of Minnesota] ProQuest Dissertations and Thesis.

Hewit. (2002). The social construction of self-esteem. In C. Snyder, \& S. Lopez (EDS.), Handbook of positive psychology (pp. 135-147). Oxford University Press.

Holder, A., Jackson, M., \& Ponterotto, J. (2015). Racial microaggression experiences and coping strategies of black women in corporate leadership. Qualitative Psychology, 2(2), 164-180.

Hollenstein, P. (2008) La reproducción de la dominación racial: experiencias de una familia Indígena en Quito. Tesis FLACSO. (38), 173-175

Iannelli, C. (2013). The role of the school curriculum in social mobility. British Journal of Sociology in Education, 34(5-6), 907-928.

http://doi.org/10.1080/01425692.2013.816031

Instituto Ecuatoriano de Estadistica y Censos. (2016). Reporte de pobreza por consumo Ecuador 2006-2014. http://www.ecuadorencifras.gob.ec/documentos/web

Johnson, B., Christensen, L. (2000). Educational research: Quantitative and qualitative approaches. Pearson Education.

Johnson, B., Onwuegbuzie, A., \& Turner, L. (2007). Toward a definition of mixed methods research. Journal of Mixed Methods Research, 1(2), 112-133.

Kessler, R., Davis, C., \& Kendler, L. (97). Childhood adversity and adult psychiatric disorder in the U.S. national comorbidity survey. Psychological Medicine, 27, 1101-1119.

Kilby, J. (2013). Introduction to special issues: Theorizing Violence. European Journal of Social Theory, 16(3), 261-272.

Klaufus, C. (2012). Urban residence: housing and social transformations in globalizing Ecuador. Berghahn Books.

Kohli, R., \& Solórzano, D. (2012). Teachers, please learn our names!: Racial microaggressions and the K-12 classroom. Race Ethnicity and Education, 15(4), 441-462. https://doi.org.10.1080/13613324.2012.674026.

Ladson-Billings, G., \& Tate, W. (1995). Toward a critical race theory of education. Teachers College Record, 97(1). 
Lantz, P., House, J., Mero, R., \& Williams, D. (2005). Stress, life events and socioeconomic disparities in health: results from the Americans' changing lives study. Journal of Health and Social Behavior, 46(3), 274-288.

Ledesma, M., \& Calderon, D. (2015). Critical Race Theory in education: A review of past literature and look to the future. Qualitative Inquiry, 21(3), 206-222.

Lewis, J., Mendenhall, R., Harwood, S., \& Browne, M. (2013). Coping with gendered racial microaggressions among black women college students. Journal of African American Students, (17), 51-73.

Liao, H., Hong, Y., \& Rounds, J. (2016). Perception of subtle racism: the role of group status and legitimizing ideologies. The Counseling Psychologist, 44 (2), 237-266.

Linnenbrink, E., \& Pintrich, P. (2003). The role of self-efficacy beliefs in student engagement and learning in the classroom. Reading and Writing Quarterly, 19(2), 119-137. https://doi.org/10.1080/10573560308223

Louise, P. (2003). A psychometric study of the Rosenberg Self-Esteem Scale: an investigation of gender. (Publication No. 13809912)[Doctoral Dissertation, The University of British Columbia]. ProQuest Dissertations and Theses Global.

Lowe, S., Okubo, Y., \& Reilly, M. (2012). A qualitative inquiry into racism, trauma, and coping: Implications for supporting victims of racism. Professional Psychology: Research and Practice, 43(3), 190-198.

Luhtanen, R., \& Crocker, J. (1992). A collective Self-Esteem Scale: Self-evaluation of one's social identity. Personality and Social Psychology Bulletin 18(3), 302-318.

Miller, s., \& Kirkland, D. (2010). Change matters. Peter Lang Publishing.

Macedo. D. (2000). Introduction. In P. Freire (Ed.). Pedagogy of the oppressed. Continuum International.

Mathers, N., Fox, N., \& Hunn, A. (2007). Surveys and Questionnaires. The National Institute for Health Research. https://www.rds-yh.nihr.ac.uk/wpcontent/uploads/2013/05/12

McCullagh, J. (2007). Racial microaggressions, college self-efficacy, and the persistence of students of color in predominantly white, four-year institutions of higher education. (Publication No. 10277694) [Doctoral dissertation, University of North Dakota]. https://commons.und.edu/cgi/viewcontent.cgi?article=2931\&context=theses 
McKay, R., Breslow, M., Sangster, R., Gabbard, S., Reynolds, R., \& Nakamoto, J. T. (1996). Translating survey questionnaires: lessons learned. In M. Braverman, \& J. Slater, Advances in Survey Research (Vol. 70, pp. 93-104). John Wiley and Sons.

Mann, M., Hosman, C., \& Schaalma, N. (2004). Self-esteem in a broad-spectrum approach for mental health promotion. Health Education Research, 19(4), 357372.

Martinez, A. (2002). The perils of poverty. In D. Narayan, \& Petesch, Voices of the poor from many lands (pp. 395-420). Oxford University Press and World Bank.

Martínez, C., \& de la Torre, C. (2010). Racial discrimination and citizenship in Ecuador educational system. Latin American and Caribbean Ethnic Studies, 5(1), 1-26.

Maslow, A. (1954). Motivation and Personality. Harper \& Row Publishers.

Mazzocco, P. (2017). The Psychology of racial colorblindness: A critical review. Palgrave Macmillan. https://10.1057/978-1-137-59302-3

Mohammad, A. (2010). Relationship between self-esteem and academic achievement amongst pre-university students. Journal of Applied Sciences, 10(20), 2474-2477

Morse, J., \& Field, P. (1995). Qualitative methods for health professionals (2nd ed.). Sage Publication.

Moore, K. M. (2012). The mental health implications of experiencing racial/ethnic microaggressions among Latina/os: Cognitive, affective, and behavioral components. (Publication No. 3523271). [Doctoral Dissertation, Marquette University]. ProQuest Dissertations and Theses Global.

Mueller, J. (2017). Producing colorblindness: everyday mechanisms of white ignorance. Social Problems (64), 219-238. https://doi.org/10.1093/socpro/spx012

Murphy, C., Stosny, S., \& Morrel, T. (2005). Self-esteem and emotional health in adolescents-gender and age as potential moderators. Journal of Family Violence, (53), 483-489.

Nadal, K., Griffin, K., Wong, Y., \& Davidoff, K. D. (2017). The injurious relationship between racial microaggressions and physical health: Implications for social work. Journal of Ethnic \& Cultural Diversity in Social Work, 26(1-2), 6-17.

Nadal, K., Wong, Y., Griffin, K., \& Sriken, J. (2014). The adverse impact of racial microaggressions on college students' self-esteem. Journal of College Student Development, 55(5), 461-474. 
Nadal, K. (2008). Preventing racial, ethnic, gender, sexual minority, disability, and religious microaggressions: Recommendations for promoting positive mental health. Prevention in Counseling Psychology: Theory, Research, Practice and Training, (2), 2-27.

Nadal, K. (2011). The racial and ethnic microaggressions scale (REMS): Construction, reliability, and validity. American Psychological Association, 58(4), 470-480.

Nadal, K. G., Wong, Y., Hamit, S., \& Rasmus, M. (2014). The impact of racial microaggressions on mental health: counseling implications for clients of color. Journal of Counseling \& Development, (92), 52-66.

Neurath, O. (1973). The scientific conception of the world: The Vienna Circle. In M. Neurath, \& R. Cohen, Empiricism and sociology. Reidel.

Nichols, L. (2007). Self-efficacy of first-generation college students and the relationship to academic performance and college adjustment. Journal of College Counseling, 10(1), 6-18.

Novo, C., \& De la Torre, C. (2010). Racial discrimination and citizenship in Ecuador's educational system. Latin American and Caribbean Ethnic Studies, 5(1), 1-26.

Ong, A., Burrow, A., Fuller-Rowel, T., Ja, N., \& Sue, D. (2013). Racial microaggressions and daily well-being among Asian Americans. Journal of Counseling Psychology, 60(2), 188-199.

Ormrod, J., Anderman, E., \& Anderman, L. (2014). Educational Psychology: Developing learners. Pearson.

Oviedo, A., \& Wildemeersch, D. (2008). Intercultural education and curricular diversification: The case of Ecuadorian intercultural bilingual education model. $A$ Journal of Comparative Education (38), 455-470.

Ozoling, U. (2009). Back translation as a means of giving translators a voice. The International Journal for Translation \& Interpreting Research, (1)2.

Pearlin, L., \& Schooler, C. (1978). The structure of coping. Journal of Health and Social Behavior, (19), 2-21.

Pedditzi, M., \& Passarelli, M. (2018). School social context, students' self-efficacy and satisfaction in high school. The Open Psychology Journal (11), 249-260.

Perez, L., \& Solórzano, D. (2015). Racial microaggressions as a tool for critical race research. Race, Ethnicity and Education, 18(3), 297-320. 
Pierce, C. (1969). Is bigotry the basis of the medical problem of the ghetto? In J. Norman, Medicine in the Ghetto (pp. 301-314). Meredith Corporation.

Pierce, C. (1970). Offensive mechanisms. In F. Barbour (Eds.), The Black seventies (pp.265-282). Porter Sargent.

Pierce, C. (1988). Stress in the workplace. In A. Coner-Edwards, \& J. Spurlock, Black families in crisis: The middle class (pp. 27-34). Brunner/Mazel.

Pierce, C., Carew, J., Pierce-Gonzalez, D., \& Willis, D. (1978). An experiment in racism: TV commercials. In C. Pierce, Television and education (pp. 62-88). Sage.

Pizarro, M., Kohli, R. I stopped sleeping: Teachers of color and the impact of racial battle fatigue. Urban Education.(1)25.

Post, D. (2011, September 2018). Ecuador: Who benefits from free higher education?

University World News.

https://www.universityworldnews.com/post.php?story=20110916210900699.

Prus, R. (1996). Symbolic interaction and ethnographic research: Intersubjectivity and the study of human lived experience. State University of New York Press.

Rahman, S. (2017). The advantages and disadvantages of using quantitative and qualitative approaches and methods in language testing and assessment research: a literature review. Journal of Education and Learning, 6(1), 102-113.

Reay, D., \& Vincent, C., Brown, P (2013). Education and Social Mobility. British Journal of Sociology of Education, 34(5-6), 637-643.

https://doi.org/10.1080/01425692.2013.826414

Reid, E. (2017). How racial microaggressions impact the mental health of black women of different occupational prestige. [Doctoral Dissertation, University of Massachusetts]

https://scholarworks.umb.edu/cgi/viewcontent.cgi?article=1026\&context=honors_ theses

Reja, U., Lozar, K., Hlebec, V., \& Vehovar, V. (2003). Open-ended vs. close-ended questions in web questionnaires. Developments in Applied Statistics, 159-177.

Rice, K., Ashby, J. \& Slaney, R. (1998). Self-Esteem as a mediator between perfectionism and depression: A structural equations analysis. American Psychological Association, 45(3), 304-314. 
Rodgers, W., Wilson, P., Hall, C., Fraser, S., \& Murray, T. (2008). Evidence of a multidimensional self-efficacy for exercise scale. Research Quarterly for Exercise and Sport, 79(2), 1-13.

Roitman, K., Oviedo, A. (2017) Mestizo racism in Ecuador. Ethnic and Racial Studies, (40)15. https://doi.ort/10.1080/01419870.2016.1260749

Rojas, C., Zegers, B., \& Foster, C. (2009). La escala de autoestima de Rosenberg: Validacion para Chile en una muestra de jóvenes adultos, adultos y adultos mayores. Revista de Medicina Chile(137), 791-800.

Rollock, D., \& Gordon, E. (2000). Racism and mental health into the 21st century: perspectives and parameters. American Journal of Orthopsychiatry, (70) 5-13.

Rosenberg, M. (1965). Society and the adolescent self-image. Princeton University Press.

Rumbaugh, N., Mitchell, C., \& Spicer, P. (2009). A longitudinal study of self-esteem, cultural identity, and academic success among American Indian adolescents. Cultural Diversity \& Ethnic Minority Psychology, 15(1), 38-50.

Salazar, C. (2009). Strategies to survive and thrive in academia: The collective voices of counseling faculty of color. International Journal of Advancement Counselling (31), 181-198.

Saldaña, J. (2009). The coding manual for qualitative researchers. Sage.

Samuel, M., Park, J. (2015). The continuing significance of racism in lives of Asian American college students. Journal of College Student Development, 56(6), 551569.

Sánchez, J. (2006). Inequality, ethnicity and social disorder: the Ecuadorian case. III World Bank Conference on Inequality: Inequality, politics, and power. Washington, DC.: World Bank.

Sánchez-Hucles, J. (1999). Racism: Emotional abusiveness and psychological trauma for ethnic minorities. Journal of emotional abuse, 1 (2), 69-87.

Santillana, A. (2013). Indagaciones sobre lo Indio, campo intelectual mestizo, intelectuales organicos e intelectuales Indígenas en el contexto ecuatoriano. CLACSO. http://biblioteca.clacso.edu.ar/clacso/becas/20131024070835/Santillanatrabajofina 1.pdf

Sarango, L. (2016, Enero-Abril). Educación Indígena propia: lo que pudo haber hecho un gobierno de "izquierda" lo hizo el gobierno de la derecha. Amawtay revista digital 
de la pluriversidad "amawtay wasi", 2(4). https://amawtaywasi.org/wpcontent/uploads/2018/08/AMAWTAY-4-final-peque.pdf

Sears, D. (1988). Symbolic racism. In K. Phyllis, \& T. Dalmas, Eliminating Racism: Profiles in controversy. Plenum Press.

Secretaria Técnica del Frente Social (2005). Racismo y discriminación racial en Ecuador. Sistema de indicadores sociales del pueblo Afroecuatoriano. SISPAE.

Seidman, I. (2013). Interviewing as qualitative research. Teachers College Press.

Sedikides, C., Gress, A. (2003). Portraits of the self. In M. A. Hogg \& J. Cooper (Eds.), Handbook of Social Psychology. Sage.

Schunk, D. (2012). Learning theories: an educational perspective. Pearson Education.

Schunk, D., \& Mullen, C. (2012). Self-efficacy as an engaged learner. In S. Christenson, A. Reschly, \& C. Wylie, Handbook of research on student engagement (pp. 219235). Stringer.

Schwarzer, R., \& Jerusalem, M. (1995). Generalized Self-Efficacy Scale. In J. Weinman, S. Wright, \& M. Johnston, Measures in health psychology: A user's portfolio. Casual and control beliefs (pp. 35-37).NFER-NELSON.

Sedikides, C., \& Gress, A. (2003). Portraits of the self. In M. Hogg, \& J. Cooper, Sage handbook of social psychology (pp. 110-138). Sage.

Seidman, I. (2013). Interviewing as qualitative research (4 $4^{\text {th }}$ ed.).Teachers College Press.

Shakouri, N. (2014). Qualitative research: incredulity toward metanarrativeness. Journal of Education and Human Development, 3(2), 671-680.

Sigler, R. (1995). The cost of tolerance for violence. Journal of Health are of the Poor and Underserved, 6(2), 124-134.

Sinclair, S., Gansler, D., Blais, M., Sandberg, E., Bistis, K., LoCicero, A. (2010). Psychometric properties of the Rosenberg Self-Esteem Scale: Overall and across demographic groups living within the United States. Evaluation \& The Health Professions, 33(1), 56-80.

Smith, L., \& Mao, S. D. (2016). Talking across worlds: Classist microaggressions and higher education. Journal of Poverty, 20(2), 127-151. 
Smith, M. (2017). Relationships among race, racial microaggressions, academic motivation, and academic self-efficacy. (Publication No. 10277694) [Masters Thesis, Texas A\&M University]. ProQuest Dissertations \& Theses Global.

Solórzano. (1997). Images and words that wound: Critical race theory, racial stereotyping, and teacher education. Teacher Education Quarterly, 5(19), 5-19.

Solórzano, D., Ceja, M., \& Yosso, T. (2000). Critical race theory, racial microaggressions, and campus racial climate: The experiences of African American college students. Journal of Negro Education, (69), 60-73.

Son, J. (2018). Back translation as a documentation tool. The International Journal for Translation \& Interpreting Research, 10(2), 89-100.

Sue, D. (2010). Microaggressions and marginality: Manifestation, dynamics, and impact. Wiley \& Sons.

Sue, D. Capodilupo, C., Torino, G., Bucceri, J., Holder, A., Nadal, K., \& Esquilin, M. (2007). Racial microaggressions in everyday life: implications for clinical practice. American Psychological Association, 62(4), 271-286.

Sue, D., Nadal, K., Capodilupo, C., Lin, A., Torino, G., Rivera, D. (2008). Racial microaggressions against Black Americans: Implications for counseling. Journal of Counseling \& Development, (86), 330-338.

Sweetman, D., Badiee, M., Creswell, J. (2010). Use of the transformative framework in mixed methods studies. Quality Inquiry, 16 (6), 441-454.

Tavakol, M., \& Zeinaloo, A. (2004). Medical research paradigms: positivistic inquiry paradigm versus naturalistic inquiry paradigm. Journal of Medical Education, (5), $75-81$.

Tawa, J., Suyemoto, K., \& Roemer, L. (2012). Implications of perceived interpersonal and structural racism for Asian Americans' self-esteem. Basic and Applied Social Psychology, 34(4), 349-358.

Thai, C., Lyons, H., Lee, M., \& Iwasaki, M. (2017). Microaggressions and self-esteem in emerging Asian American adults: the moderating role of racial socialization. Asian American Journal of Psychology, 8 (2), 83-93.

Tran, A., \& Lee, R. (2010). Perceived ethnic-racial socialization, ethnic identity, and social competence among Asian American late adolescents. Cultural Diversity \& Ethnic Minority Psychology, 16 (2), 169-178. 
Umaña, A., \& Updegraff, K. (2007). Latino adolescents' mental health: Exploring the interrelations among discrimination, ethnic identity, cultural orientation, selfesteem, and depressive symptoms. Journal of Adolescence, (30), 549-567.

Universidad San Francisco de Quito. (2017). Micro Agresiones. Revista Enfoque (41). USFQ.

https://www.usfq.edu.ec/publicaciones/enfoque/Documents/2017/enfoque_2017_ 07.pdf.

Vaccaro, A. (2010). What lies beneath seemingly positive campus climate results: institutional sexism, racism, and male hostility toward equity initiatives and liberal bias. Equity \& Excellence in Education, 43(2), 202-215.

VandenBos, G. (2015). APA dictionary of Psychology. American Psychological Association.

Wang, J., Leu, J., \& Shoda, Y. (2011). When the seemingly innocuous "stings": Racial microaggressions and their emotional consequences. Personality and Social Psychology Bulletin, 20(10), 1-13.

Weynand, T. (2016, June 29). Cultural Imperialism. Encyclopedia Britannica. https://www.britannica.com/topic/cultural-imperialism.

Williams, D., \& Rhodes, R. (2014). The confounded self-efficacy construct: review, conceptual analysis, and recommendations for future research. Health Psychology review, 10(2), 113-128.

Williams, D., Neighbors, H., \& Jackson, J. (2008). Racial/ethnic discrimination and health: findings from community studies. American Journal of Public Health, 98(1), 29-37.

Wong, G., Derthick, A., David, E., Saw, A., \& Okazaki, S. (2014). The what, the why, and the wow: A review of racial microaggressions research in Psychology. Race and Social Problems, (3),1-21.

Wright, N. (2016). An exploration of sense of belonging among black males attending a predominately white institution. [Masters Theses, Eastern Illinois University]. https://thekeep.eiu.edu/cgi/viewcontent.cgi? article=3472\&context=theses

Yeung, K. \& Martin, J. (2003. The looking glass self: An empirical test and elaboration forces. Social Forces, 81(3), 843-879.

Yosso, T. (2005). Whose culture has capital? A critical race theory discussion of community cultural wealth. Race, Ethnicity and Education, 8(1), 69-91. 
Young, I. (2004). The five faces of oppression. In L. Heldke, \& P. O’Connor, Oppression, privilege, \& resistance: Theoretical perspectives on racism, sexism, and heterosexism (pp. 37-63). McGraw-Hill.

Young, J., Nguyen, D., \& Block, C. (2018). The 360-degree experience of workplace microaggressions: Who commits them? How do individuals respond? What are the consequences? In G. Torini, D. Rivera, C. Capodilupo, K. Nadal, \& D. Sue, Microaggression theory: Influence and implications (pp. 159-177). John Willey \& Sons, Inc.

Zimmerman, B. (1995). Self-efficacy and educational development. In A. Bandura, Selfefficacy in changing societies (pp. 202-231). Cambridge University Press.

Zoabi, K. (2012). Self-esteem and motivation for learning among minority students: a comparison between students of pre-academic and regular programs. Creative Education, 3(8), 1-7.

Zoller, M., \& Gerard, J. (2011). Self-esteem and academic achievement: a comparative study of adolescent students in England and the United States. Compare: A Journal of Comparative and International Education, 41(5), 629-648. 


\section{APPENDIX A}

\section{RACIAL MICROAGGRESSIONS SURVEY ORIGINAL VERSION BY COMPONENTS}

Constructs included in the survey:

- Assumptions of inferiority

- Microinvalidations

- Assumptions of second class

- Exocitization of Indigenous people

- Strategies to cope with microaggressions

Components of the survey:

- Racial microaggressions questionnaire

- Student Demographic information 


\section{Racial and Ethnic Microaggressions Scale Original Version by Components}

Part 1. Instructions: Please think about your experiences as a student and indicate the number of times that the described events occurred to you in the last 6 months. Rate your answers as follows:

$5=\mathrm{I}$ experienced this event 10 or more times in the past six months

$4=\mathrm{I}$ experienced this event 7-9 times in the past six months

$3=\mathrm{I}$ experienced this event $4-6$ times in the past six months

$2=\mathrm{I}$ experienced this event 1-3 times in the past six months

1 = I did not experienced this event in the past six months

\begin{tabular}{|c|c|c|c|c|c|c|}
\hline \multicolumn{7}{|c|}{ Construct 1: Assumptions of Inferiority } \\
\hline & & $\begin{array}{l}\text { I experienced } \\
\text { this event } 10 \\
\text { or more } \\
\text { times in the } \\
\text { past six } \\
\text { months } \\
5\end{array}$ & $\begin{array}{l}\text { I experienced } \\
\text { this event 7-9 } \\
\text { times in the } \\
\text { past six } \\
\text { months } \\
4\end{array}$ & $\begin{array}{l}\text { I experienced } \\
\text { this event } 4-6 \\
\text { times in the } \\
\text { past six } \\
\text { months } \\
3\end{array}$ & $\begin{array}{l}\text { I experienced } \\
\text { this event } 1-3 \\
\text { times in the } \\
\text { past six } \\
\text { months } \\
2\end{array}$ & $\begin{array}{c}\text { I did not } \\
\text { experience } \\
\text { this event in } \\
\text { the past six } \\
\text { months } \\
1\end{array}$ \\
\hline$\underline{32}$ & $\begin{array}{l}\text { Someone assumed } \\
\text { that I would have a } \\
\text { lower education } \\
\text { because of my } \\
\text { race/ethnicity. }\end{array}$ & & & & & \\
\hline 38 & $\begin{array}{l}\text { Someone assumed } \\
\text { that I was poor } \\
\text { because of my } \\
\text { race/ethnicity. }\end{array}$ & & & & & \\
\hline 21 & $\begin{array}{l}\text { Someone assumed } \\
\text { that I would not be } \\
\text { educated because of } \\
\text { my race/ethnicity. }\end{array}$ & & & & & \\
\hline 17 & $\begin{array}{l}\text { Someone acted } \\
\text { surprised at my } \\
\text { scholastic or } \\
\text { professional success } \\
\text { because of my } \\
\text { race/ethnicity. }\end{array}$ & & & & & \\
\hline 9 & $\begin{array}{l}\text { Someone assumed } \\
\text { that I would not be } \\
\text { intelligent because } \\
\text { of my race/ethnicity. }\end{array}$ & & & & & \\
\hline 36 & $\begin{array}{l}\text { Someone assumed } \\
\text { that I held a lower } \\
\text { paying job because } \\
\text { of my race/ethnicity. }\end{array}$ & & & & & \\
\hline
\end{tabular}




\begin{tabular}{|l|l|l|l|l|l|l|}
\hline 5 & $\begin{array}{l}\text { Someone assumed } \\
\text { that I grew up in a } \\
\text { particular } \\
\text { neighborhood } \\
\text { because of my } \\
\text { race/ethnicity }\end{array}$ & & & & & \\
\hline 22 & $\begin{array}{l}\text { Someone told me } \\
\text { that I was } \\
\text { "articulate" after } \\
\text { she/he assumed I } \\
\text { wouldn't be. }\end{array}$ & & & & & \\
\hline
\end{tabular}

\begin{tabular}{|c|c|c|c|c|c|c|}
\hline \multicolumn{7}{|c|}{ Construct 2: Second-Class Citizenry and Assumptions of Criminality } \\
\hline & & $\begin{array}{l}\text { I experienced } \\
\text { this event } 10 \\
\text { or more } \\
\text { times in the } \\
\text { past six } \\
\text { months } \\
5\end{array}$ & $\begin{array}{l}\text { I experienced } \\
\text { this event } 7-9 \\
\text { times in the } \\
\text { past six } \\
\text { months } \\
4\end{array}$ & $\begin{array}{l}\text { I experienced } \\
\text { this event } 4-6 \\
\text { times in the } \\
\text { past six } \\
\text { months } \\
3\end{array}$ & $\begin{array}{l}\text { I } \\
\text { experienced } \\
\text { this event 1- } \\
3 \text { times in } \\
\text { the past six } \\
\text { months } \\
2\end{array}$ & $\begin{array}{c}\text { I did not } \\
\text { experience } \\
\text { this event } \\
\text { in the past } \\
\text { six } \\
\text { months } \\
1\end{array}$ \\
\hline 6 & $\begin{array}{l}\text { Someone avoided } \\
\text { walking near me on the } \\
\text { street because of my } \\
\text { race/ethnicity. }\end{array}$ & & & & & \\
\hline 31 & $\begin{array}{l}\text { Someone clenched } \\
\text { her/his purse or walled } \\
\text { upon seeing me } \\
\text { because of my } \\
\text { race/ethnicity. }\end{array}$ & & & & & \\
\hline 8 & $\begin{array}{l}\text { Someone avoided } \\
\text { sitting next to me in a } \\
\text { public space (e.g., } \\
\text { restaurants, movie } \\
\text { theaters, subways, } \\
\text { buses) because of my } \\
\text { race/ethnicity. }\end{array}$ & & & & & \\
\hline 40 & $\begin{array}{l}\text { Someone avoided eye } \\
\text { contact with me } \\
\text { because of my } \\
\text { race/ethnicity. }\end{array}$ & & & & & \\
\hline 2 & $\begin{array}{l}\text { Someone's body } \\
\text { language showed they } \\
\text { were scared of me, } \\
\text { because of my } \\
\text { race/ethnicity. }\end{array}$ & & & & & \\
\hline 34 & $\begin{array}{l}\text { Someone assumed that } \\
\text { I would physically hurt }\end{array}$ & & & & & \\
\hline
\end{tabular}




\begin{tabular}{|l|l|l|l|l|l|}
\hline & $\begin{array}{l}\text { them because of my } \\
\text { race/ethnicity. }\end{array}$ & & & & \\
\hline 11 & $\begin{array}{l}\text { I received substandard } \\
\text { service in stores } \\
\text { compared to customers } \\
\text { of other racial groups. }\end{array}$ & & & & \\
\hline
\end{tabular}

\begin{tabular}{|c|c|c|c|c|c|c|}
\hline \multicolumn{7}{|c|}{ Construct 3: Microinvalidations } \\
\hline & & $\begin{array}{l}\text { I experienced } \\
\text { this event } 10 \\
\text { or more times } \\
\text { in the past six } \\
\text { months } \\
5\end{array}$ & $\begin{array}{c}\text { I } \\
\text { experienced } \\
\text { this event } 7- \\
9 \text { times in } \\
\text { the past six } \\
\text { months } \\
4\end{array}$ & $\begin{array}{l}\text { I experienced } \\
\text { this event } 4-6 \\
\text { times in the } \\
\text { past six } \\
\text { months } \\
\quad 3\end{array}$ & $\begin{array}{l}\text { I experienced } \\
\text { this event } 1-3 \\
\text { times in the } \\
\text { past six } \\
\text { months } \\
2\end{array}$ & $\begin{array}{c}\text { I did not } \\
\text { experience } \\
\text { this event } \\
\text { in the past } \\
\text { six } \\
\text { months } \\
1\end{array}$ \\
\hline 27 & $\begin{array}{l}\text { Someone told me that } \\
\text { they "don't see color." }\end{array}$ & & & & & \\
\hline 30 & $\begin{array}{l}\text { Someone told me that } \\
\text { they do not see } \\
\text { race/ethnicity. }\end{array}$ & & & & & \\
\hline 39 & $\begin{array}{l}\text { Someone told me that } \\
\text { people should not } \\
\text { think about } \\
\text { race/ethnicity } \\
\text { anymore. }\end{array}$ & & & & & \\
\hline 7 & $\begin{array}{l}\text { Someone told me that } \\
\text { she or he was color- } \\
\text { blind. }\end{array}$ & & & & & \\
\hline 26 & $\begin{array}{l}\text { I was told that people } \\
\text { of color do not } \\
\text { experience racism } \\
\text { anymore. }\end{array}$ & & & & & \\
\hline 33 & $\begin{array}{l}\text { Someone of a different } \\
\text { racial group has stated } \\
\text { that there is no } \\
\text { difference between the } \\
\text { two of us. }\end{array}$ & & & & & \\
\hline 4 & $\begin{array}{l}\text { I was told that I should } \\
\text { not complain about } \\
\text { race/ethnicity. }\end{array}$ & & & & & \\
\hline 14 & $\begin{array}{l}\text { I was told that people } \\
\text { of all racial/ethnic } \\
\text { groups experience the } \\
\text { same obstacles. }\end{array}$ & & & & & \\
\hline 10 & $\begin{array}{l}\text { I was told that I } \\
\text { complain about race } \\
\text { too much. }\end{array}$ & & & & & \\
\hline
\end{tabular}




\begin{tabular}{|c|c|c|c|c|c|c|}
\hline \multicolumn{7}{|c|}{ Construct 4: Exoticization/Assumptions of Similarity } \\
\hline & & $\begin{array}{l}\text { I experienced } \\
\text { this event } 10 \\
\text { or more times } \\
\text { in the past six } \\
\text { months } \\
5\end{array}$ & $\begin{array}{l}\text { I experienced } \\
\text { this event 7-9 } \\
\text { times in the } \\
\text { past six } \\
\text { months } \\
4\end{array}$ & $\begin{array}{l}\text { I experienced } \\
\text { this event 4-6 } \\
\text { times in the } \\
\text { past six } \\
\text { months } \\
3\end{array}$ & $\begin{array}{l}\text { I experienced } \\
\text { this event } 1-3 \\
\text { times in the } \\
\text { past six } \\
\text { months } \\
2\end{array}$ & $\begin{array}{l}\text { I did not } \\
\text { experience } \\
\text { this event in } \\
\text { the past six } \\
\text { months } \\
1\end{array}$ \\
\hline 3 & $\begin{array}{l}\text { Someone assumed } \\
\text { that I spoke a } \\
\text { language other than } \\
\text { Spanish because of } \\
\text { my race/ethnicity. }\end{array}$ & & & & & \\
\hline 29 & $\begin{array}{l}\text { Someone asked me to } \\
\text { teach them words in } \\
\text { my "native } \\
\text { language." }\end{array}$ & & & & & \\
\hline 45 & $\begin{array}{l}\text { Someone assumed } \\
\text { that I speak similar } \\
\text { languages to other } \\
\text { people in my race. }\end{array}$ & & & & & \\
\hline 35 & $\begin{array}{l}\text { Someone assumed } \\
\text { that I ate foods } \\
\text { associated with my } \\
\text { race/culture every } \\
\text { day. }\end{array}$ & & & & & \\
\hline 42 & $\begin{array}{l}\text { Someone told me that } \\
\text { all people in my } \\
\text { racial group look } \\
\text { alike. }\end{array}$ & & & & & \\
\hline 23 & $\begin{array}{l}\text { Someone told me that } \\
\text { all people in my } \\
\text { racial group are all } \\
\text { the same. }\end{array}$ & & & & & \\
\hline 13 & $\begin{array}{l}\text { Someone wanted to } \\
\text { date me only because } \\
\text { of my race. }\end{array}$ & & & & & \\
\hline 20 & $\begin{array}{l}\text { Someone did not } \\
\text { believe me when I } \\
\text { told them I was born } \\
\text { in the U.S. }\end{array}$ & & & & & \\
\hline 43 & $\begin{array}{l}\text { Someone objectified } \\
\text { one of my physical } \\
\text { features because of } \\
\text { my race/ethnicity. }\end{array}$ & & & & & \\
\hline
\end{tabular}




\begin{tabular}{|c|c|c|c|c|c|c|}
\hline \multicolumn{7}{|c|}{ Construct 5: Environmental Microaggressions } \\
\hline & & $\begin{array}{c}\text { I experienced } \\
\text { this event } 10 \\
\text { or more times } \\
\text { in the past six } \\
\text { months } \\
5\end{array}$ & $\begin{array}{c}\text { I } \\
\text { experienced } \\
\text { this event } \\
7-9 \text { times in } \\
\text { the past six } \\
\text { months } \\
4\end{array}$ & $\begin{array}{l}\text { I } \\
\text { experienced } \\
\text { this event } \\
4-6 \text { times in } \\
\text { the past six } \\
\text { months } \\
\quad 3\end{array}$ & $\begin{array}{c}\text { I } \\
\text { experienced } \\
\text { this event } \\
1-3 \text { times in } \\
\text { the past six } \\
\text { months } \\
2\end{array}$ & $\begin{array}{l}\text { I did not } \\
\text { experience } \\
\text { this event } \\
\text { in the past } \\
\text { six } \\
\text { months } \\
1\end{array}$ \\
\hline 37 & $\begin{array}{l}\text { I observed } \\
\text { people of my } \\
\text { race/ethnicity } \\
\text { portrayed } \\
\text { positively in } \\
\text { movies. }\end{array}$ & & & & & \\
\hline 24 & $\begin{array}{l}\text { I observed } \\
\text { people of my } \\
\text { race/ethnicity } \\
\text { portrayed } \\
\text { positively in } \\
\text { magazines. }\end{array}$ & & & & & \\
\hline 19 & $\begin{array}{l}\text { I observed } \\
\text { people of my } \\
\text { race/ethnicity } \\
\text { portrayed } \\
\text { positively on } \\
\text { television. }\end{array}$ & & & & & \\
\hline 28 & $\begin{array}{l}\text { I read popular } \\
\text { books or } \\
\text { magazines in } \\
\text { which a } \\
\text { majority of } \\
\text { contributions } \\
\text { featured people } \\
\text { from my racial } \\
\text { group. }\end{array}$ & & & & & \\
\hline 18 & $\begin{array}{l}\text { I observed that } \\
\text { people of my } \\
\text { race/ethnicity } \\
\text { were the CEOs } \\
\text { of major } \\
\text { corporations. }\end{array}$ & & & & & \\
\hline 41 & $\begin{array}{l}\text { I observed that } \\
\text { someone of my } \\
\text { race/ethnicity is } \\
\text { a government } \\
\text { official in my } \\
\text { state. }\end{array}$ & & & & & \\
\hline
\end{tabular}




\begin{tabular}{|l|l|l|l|l|l|}
\hline 12 & $\begin{array}{l}\text { I observed } \\
\text { people of my } \\
\text { race/ethnicity in } \\
\text { prominent } \\
\text { positions at my } \\
\text { workplace or } \\
\text { school. }\end{array}$ & & & & \\
\hline
\end{tabular}

\begin{tabular}{|c|c|c|c|c|c|c|}
\hline \multicolumn{7}{|c|}{ Construct 6: Workplace and School Microaggressions } \\
\hline & & $\begin{array}{l}\text { I experienced } \\
\text { this event } 10 \\
\text { or more times } \\
\text { in the past six } \\
\text { months } \\
5\end{array}$ & $\begin{array}{l}\text { I experienced } \\
\text { this event } 7- \\
9 \text { times in the } \\
\text { past six } \\
\text { months } \\
4\end{array}$ & $\begin{array}{l}\text { I experienced } \\
\text { this event } 4- \\
6 \text { times in the } \\
\text { past six } \\
\text { months } \\
\quad 3\end{array}$ & $\begin{array}{l}\text { I } \\
\text { experienced } \\
\text { this event } 1- \\
3 \text { times in } \\
\text { the past six } \\
\text { months } \\
2\end{array}$ & $\begin{array}{l}\text { I did not } \\
\text { experience } \\
\text { this event in } \\
\text { the past six } \\
\text { months } \\
1\end{array}$ \\
\hline 25 & $\begin{array}{l}\text { An employer or co- } \\
\text { worker was } \\
\text { unfriendly or } \\
\text { unwelcoming toward } \\
\text { me because of my } \\
\text { race/ethnicity. }\end{array}$ & & & & & \\
\hline 15 & $\begin{array}{l}\text { My opinion was } \\
\text { overlooked in a group } \\
\text { discussion because of } \\
\text { my race/ethnicity. }\end{array}$ & & & & & \\
\hline 1 & $\begin{array}{l}\text { I was ignored at } \\
\text { school or at work } \\
\text { because of my } \\
\text { race/ethnicity. }\end{array}$ & & & & & \\
\hline 16 & $\begin{array}{l}\text { Someone assumed } \\
\text { that my work would } \\
\text { be inferior to people } \\
\text { of other racial groups. }\end{array}$ & & & & & \\
\hline 44 & $\begin{array}{l}\text { An employer or co- } \\
\text { worker treated me } \\
\text { differently than White } \\
\text { co-workers. }\end{array}$ & & & & & \\
\hline
\end{tabular}

Source: Nadal (2011) 


\author{
APPENDIX B \\ RECRUITMENT ADVERTISEMENT
}

|FLYER INVITING STUDENTS TO PARTICIPATE IN STUDY
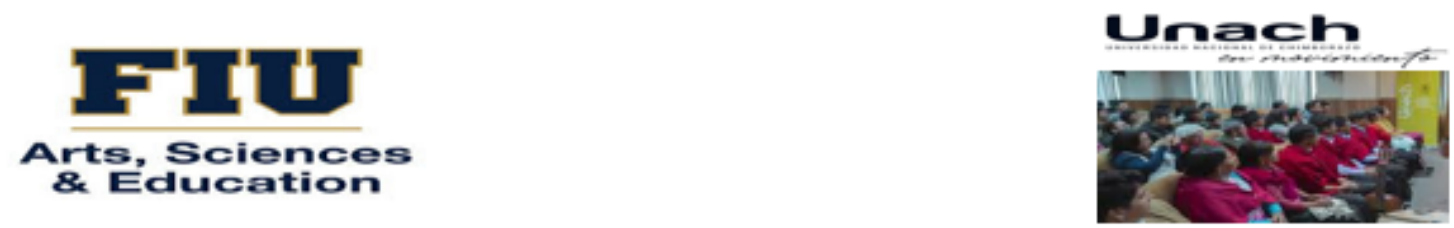

\title{
RACIAL MICROAGGRESSIONS OF INDIGENOUS STUDENTS IN AN ECUADORIAN PUBLIC HIGHER EDUCATION INSTITUTION
}

\section{INVITATION TO PARTICIPATE IN RESEARCH STUDY}

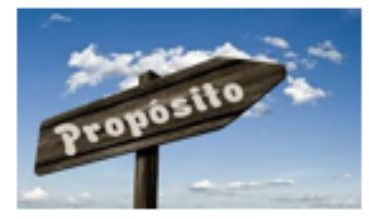

Purpose: To examine microaggressions affecting Indigenous undergraduate students pursuing a bachelor's degree in a public higher education institution in Ecuador and the associations with self-esteem and self-efficacy. To explain the experience of Indigenous students with respect to how they respond/react to and deal with microaggressions.

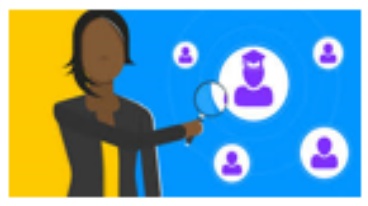

Importance: this study contributes to understand the experiences of Indigenous students with microaggressions. Explains how students experience racial hostilities to creating a welcoming environment that guarantees equal opportunities to all students.

\section{PARTICIPANTS AND TASKS}

* Students who self-identify as Indigenous enrolled in any school at the university are welcome to participate in this study.

* Participants will take three surveys. They will need approximately one hour to complete the three surveys.

* Surveys are anonymous and do not compromise your academic duties.

This studx is heine conducted bx Adriana Cundar, She is a faculty member at Universidad Nacional de Chimborazo and PhD.

Candidate at Florida International University. If you should have any questions please contact the researcher via email or phone ath. acund003@fiu.edu
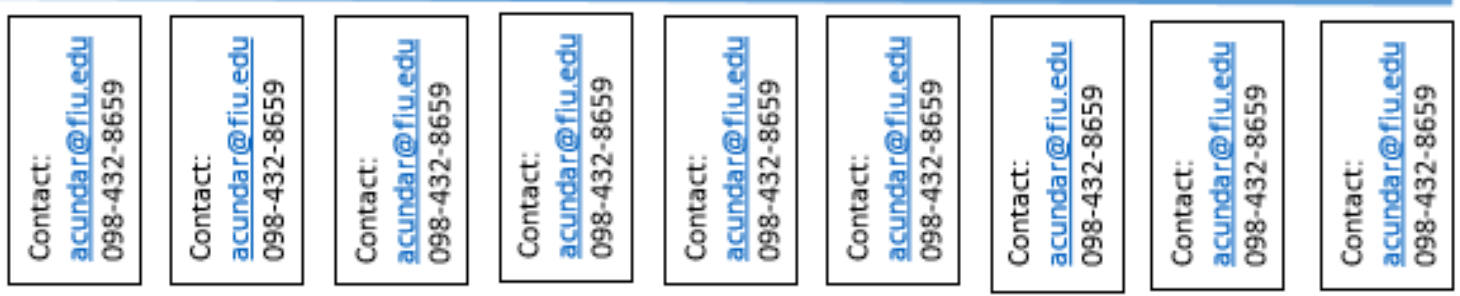


\section{VOLANTE PARA INVITAR A ESTUDIANTES A PARTICIPAR EN EL ESTUDIO \\ Unach}

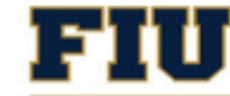

Arts, Sciences

\& Education

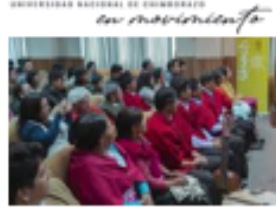

\section{MICROAGRESIONES RACIALES EN ESTUDIANTES INDÍGENAS DE PREGRADO EN UNA UNIVERSIDAD PUBLICA ECUATORIANA}

\section{INVITACION A PARTICIPAR EN ESTUDIO}

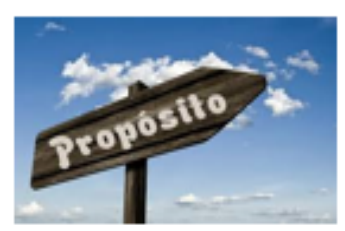

Propósito: Examinar las micro agresiones que afectan a estudiantes Indigenas que cursan sus estudios de pregrado en una institución pública de educación superior en el Ecuador y las asociaciones con su autoestima y autoeficacia. Explicar la experiencia de los estudiantes Indígenas con respecto a sus respuestas/reacciones y manejo de las micro agresiones.

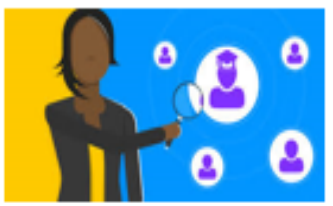

Importancia: este estudio contribuye a entender las experiencias de estudiantes Indígenas en referencia a micro agresiones. Pretende explicar las maneras en las que los estudiantes indígenas experimentan las hostilidades raciales para crear ambientes universitarios más saludables que garanticen igualdad de oportunidades para todos.

\section{QUIENES PUEDEN PARTICIPAR Y LAS TAREAS A CUMPLIR}

* Estudiantes que se auto identifiquen como Indígenas que se encuentran cursando cualquiera de las carreras de la universidad son bienvenidos a participar en este estudio.

* Los participantes llenarán tres encuestas. El tiempo aproximado para completar las tres encuestas es de una hora.

* Las encuestas son anónimas y no comprometen su actividad académica.

Este estudio está siendo desarrollado por Adriana Cundar. Docente de la Universidad Nacional de Chimborazo y candidata a PhD. en la Universidad Internacional de la horida. Si tiene preguntas puede contactar al investigador por email a: acund003@fiu.edu
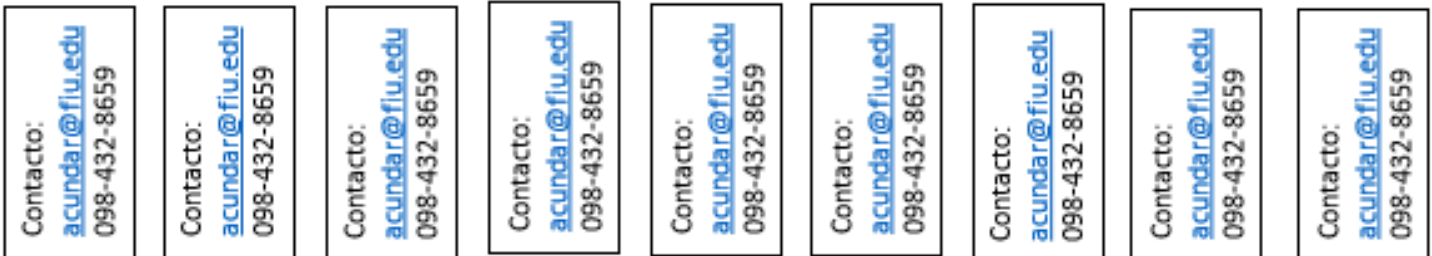


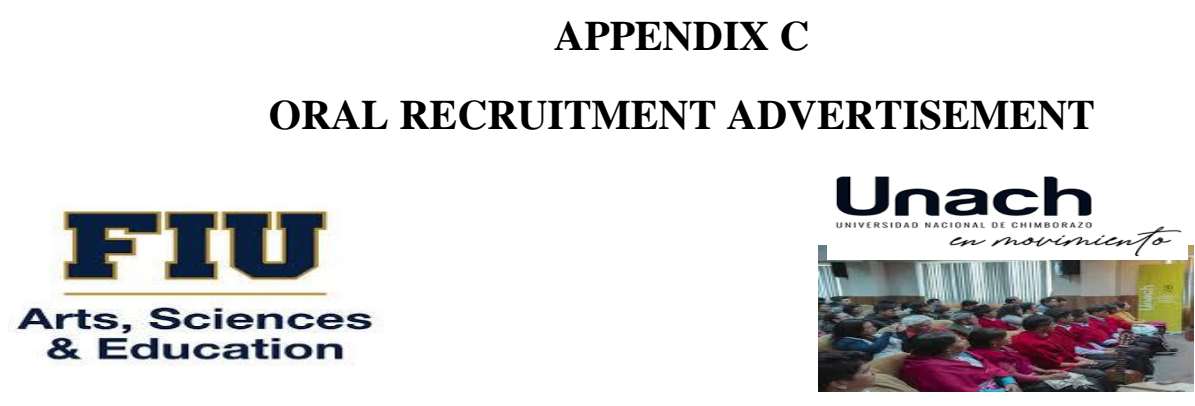

\section{Oral Invitation to Participate in Study}

I am a researcher interested in learning about racial microaggressions of Indigenous undergraduate students in an Ecuadorian higher education institution. I am a faculty member at Universidad Nacional de Chimborazo and I am currently writing my dissertation at Florida International University. For this study, I am recruiting students who identify themselves as Indigenous. Prospective participants will take three surveys related to racial microaggressions, self-esteem, and self-efficacy. The surveys will take no more than one hour and will be anonymous. There are no foreseeable risks in participating in this research. If you did participate, you will be helping the researcher understand how microaggressions manifest in Indigenous populations who pursue a university degree in a public higher education institution. I will greatly appreciate your participation in this research study. Your teacher will now pass around a sign-up sheet. If you are interested in participating, please print your name and an email address where I can best reach you. Once I receive this list I will send you an email informing you about the location, date and time the surveys will take place that will not interfere with your schedule. Thank you so much for your willingness to participate in this study. I am looking forward to contacting you soon. 

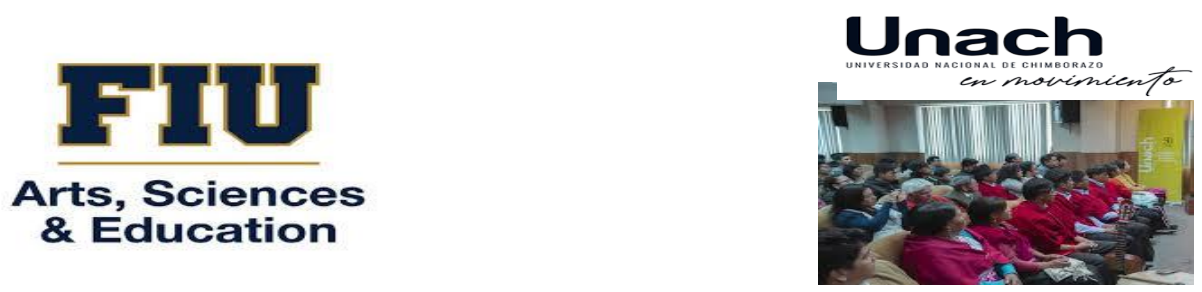

\section{Invitación Oral para Participar en Estudio}

Soy una investigadora interesada en entender sobre las micro agresiones raciales en estudiantes indígenas de pregrado en una institución de educación superior Ecuatoriana. Soy docente de la Universidad Nacional de Chimborazo y actualmente estoy escribiendo mi tesis de graduación en la Universidad Internacional de la Florida. En este momento, estoy reclutando estudiantes que se auto identifican como Indígenas para mi investigación. Los interesados en participar tomarán tres encuestas relacionadas con las micro agresiones raciales, su autoestima y su autoeficacia. Las encuestas no tomarán más de una hora y serán anónimas. No hay riesgos previsibles al participar en esta investigación. Si usted decide participar, usted estará ayudándome como investigadora a comprender cómo se manifiestan las micro agresiones en los estudiantes Indígenas que buscan obtener un título universitario en una institución pública. Aprecio enormemente su participación en este estudio de investigación. Su profesor (a) pasará una hoja de registro. Si está interesado en participar, escriba su nombre y una dirección de correo electrónico donde podamos contactarlo mejor. Una vez que yo reciba esta lista, le enviare un correo electrónico informándole sobre la ubicación, la fecha y la hora en que se realizarán las encuestas que no interferirán con su horario. Muchas gracias por su disposición a participar en este estudio. Espero contactarlos muy pronto. 
APPENDIX D

AUTHORIZATION TO CONDUCT RESEARCH

VICERRECTORADO ACADÉMICO

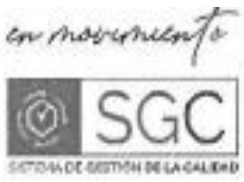

Riobamba, 24 de junio de 2019

Oficio No. 1997-V.-Académico-UNACH-2019

Doctora

Adriana Cundar

DOCENTE DE LA UNACH

Presente

De mi consideración:

En atención a su pedido formulado mediante oficio S/N de fecha 21 de junio de 2019, mediante el cual solicita autorización para el desarrollo de varias encuestas a nivel de estudiantes de nuestra institución como parte de su proyecto de tesis "Micro agresiones Raciales de Estudiantes Incigenas en una Institución Mestiza Ecuatoriana", el Vicerrectorado Académico en su afán siempre de contribuir con el avance académico de nuestros docentes, AUTORIZA su requerimiento, agradecere cocrdinar con las partes pertinentes para la ejecución de su propuesta, constituye este documento su aprobación a las encuestas solicitadas.

Particular que comunico para los fines pertinentes.

Atentamente,

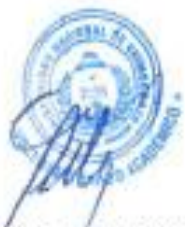

Dra. Ángela Calderón Tobar, PhD.

VICERRECTORA ACADEMICA

UNIVERSIDAD NACIONAL DE CHIMBORAZO

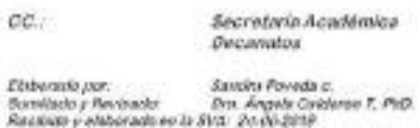




\section{APPENDIX E \\ RACIAL MICROAGGRESSIONS SURVEY RANDOMIZED ITEMS}

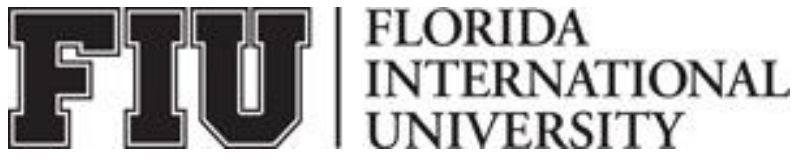

\section{Survey on Racial and Ethnic Microaggressions in Ecuadorian Public Higher Education Institutions}

Dear Participant:

Thank you for accepting to participate in this survey. The purpose of this instrument is to expand knowledge regarding racial microaggressions as experienced by Indigenous students while pursuing a college degree. We understand microaggressions as "everyday verbal, nonverbal, and environmental slights, snubs, or insults, whether intentional or unintentional, which communicate hostile, derogatory, or negative messages to target persons based solely upon their marginalized group membership" (Sue, D., 2010). This survey has been adapted to the reality of Ecuadorian Public Higher Education Institutions that host indigenous populations. The questions invite you to think about your experiences as a student on microaggressions because of your ethnicity or status as a minority group on campus. Your sincere and thoughtful answers will give voice to minority Indigenous students and will help to better understand racial issues in Ecuadorian universities.

Your participation in this study is voluntary and does not interfere with your academic work. The information you provide will be kept confidential. You are allowed to refuse to take the survey without any consequence. If you decide to participate, you will be asked to complete two parts in the questionnaire: the first explores students' experiences with microaggressions and the second gathers demographic information. No risks are identified for participants taking this questionnaire.

Please read each question carefully, take your time to reflect before providing an answer. Questions are based on a scale from 1 to 5. Please indicate your opinion as follows:

$5=$ I experienced this event 10 or more times in the past six months

$4=$ I experienced this event 7-9 times in the past six months

3 = I experienced this event 4-6 times in the past six months

$2=$ I experienced this event 1-3 times in the past six months

$1=\mathrm{I}$ did not experienced this event in the past six months

Please follow instructions carefully and do not hesitate to ask a question to the researcher if needed.

We greatly appreciate your collaboration. 


\section{Racial and Ethnic Microaggressions Scale}

Part 1. Instructions: Please think about your experiences as a student and indicate the number of times that the described events occurred to you in the last 6 months. Rate your answers as follows:

$5=\mathrm{I}$ experienced this event 10 or more times in the past six months

$4=$ I experienced this event 7-9 times in the past six months

$3=$ I experienced this event 4-6 times in the past six months

$2=\mathrm{I}$ experienced this event $1-3$ times in the past six months

i. = I did not experienced this event in the past six months

\begin{tabular}{|c|c|c|c|c|c|c|}
\hline & & $\begin{array}{l}\text { I experienced } \\
\text { this event } 10 \\
\text { or more times } \\
\text { in the past six } \\
\text { months } \\
5\end{array}$ & $\begin{array}{l}\text { I } \\
\text { experienced } \\
\text { this event 7- } \\
9 \text { times in } \\
\text { the past six } \\
\text { months } \\
4 \\
\end{array}$ & $\begin{array}{c}\text { I } \\
\text { experienced } \\
\text { this event 4- } \\
6 \text { times in } \\
\text { the past six } \\
\text { months } \\
3 \\
\end{array}$ & $\begin{array}{c}\text { I } \\
\text { experienced } \\
\text { this event 1- } \\
3 \text { times in } \\
\text { the past six } \\
\text { months } \\
2 \\
\end{array}$ & $\begin{array}{l}\text { I did not } \\
\text { experience } \\
\text { this event in } \\
\text { the past six } \\
\text { months } \\
1\end{array}$ \\
\hline$\underline{1}$ & $\begin{array}{l}\text { I was ignored at } \\
\text { school or at work } \\
\text { because of my } \\
\text { race/ethnicity. }\end{array}$ & & & & & \\
\hline 2 & $\begin{array}{l}\text { Someone's body } \\
\text { language showed } \\
\text { they were scared of } \\
\text { me (felt } \\
\text { uncomfortable), } \\
\text { because of my } \\
\text { race/ethnicity. }\end{array}$ & & & & & \\
\hline 3 & $\begin{array}{l}\text { Someone assumed } \\
\text { that I spoke a } \\
\text { language other than } \\
\text { Spanish because of } \\
\text { my ethnicity }\end{array}$ & & & & & \\
\hline 4 & $\begin{array}{l}\text { I was told that I } \\
\text { should not complain } \\
\text { about race/ethnicity. }\end{array}$ & & & & & \\
\hline 5 & $\begin{array}{l}\text { Someone assumed } \\
\text { that I grew up in a } \\
\text { particular } \\
\text { neighborhood or in } \\
\text { the countryside } \\
\text { because of my } \\
\text { race/ethnicity. }\end{array}$ & & & & & \\
\hline
\end{tabular}









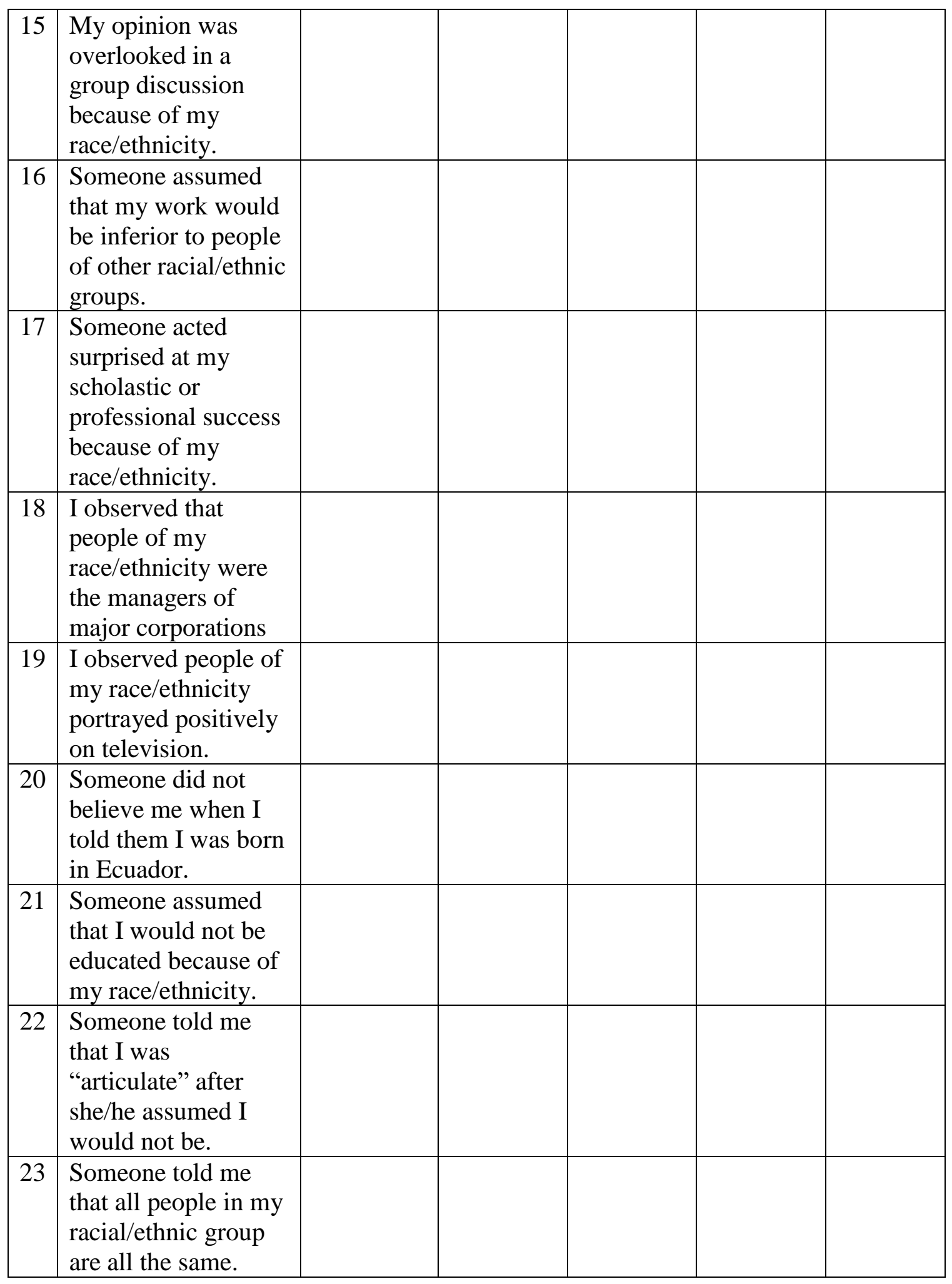




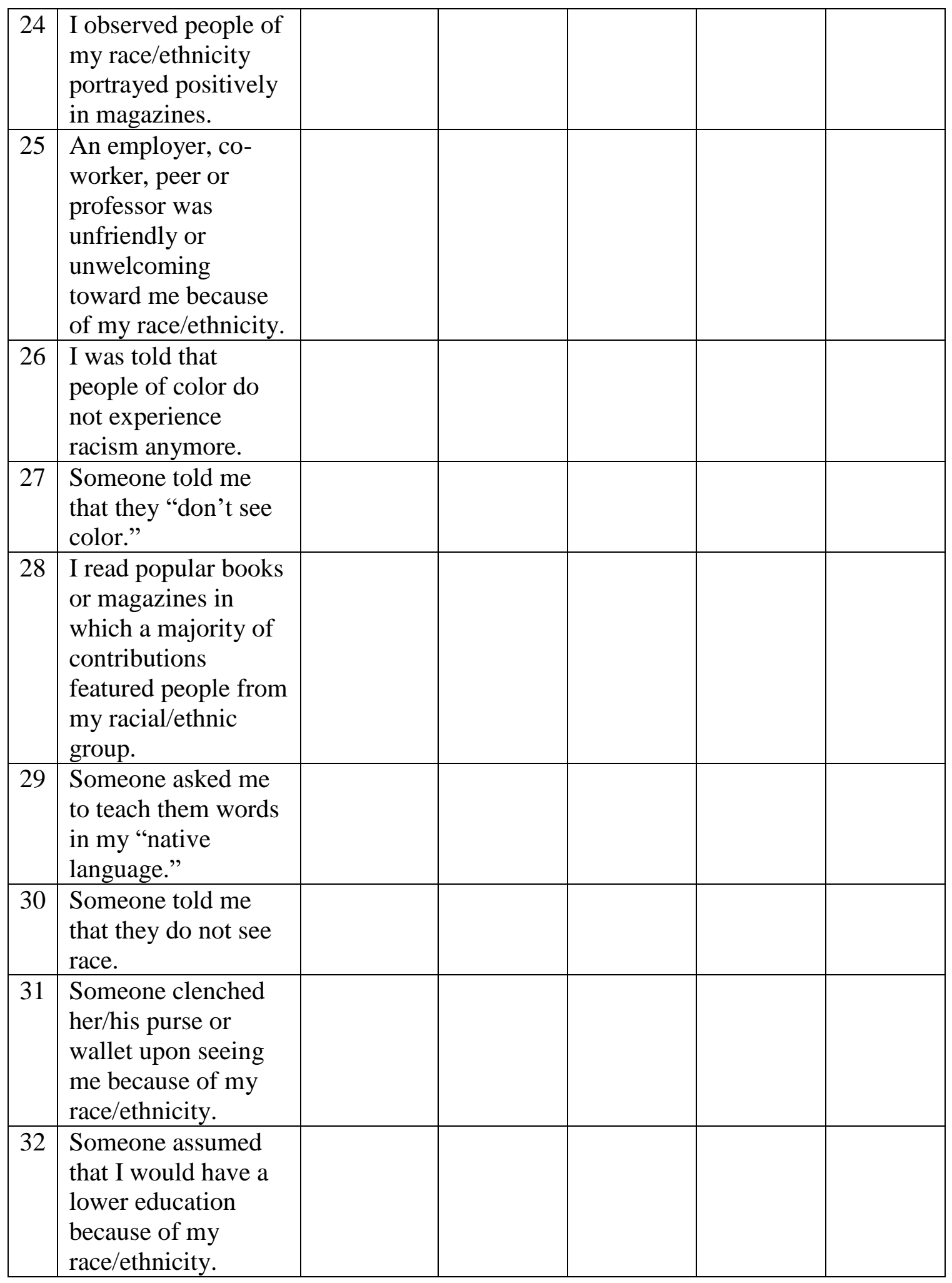









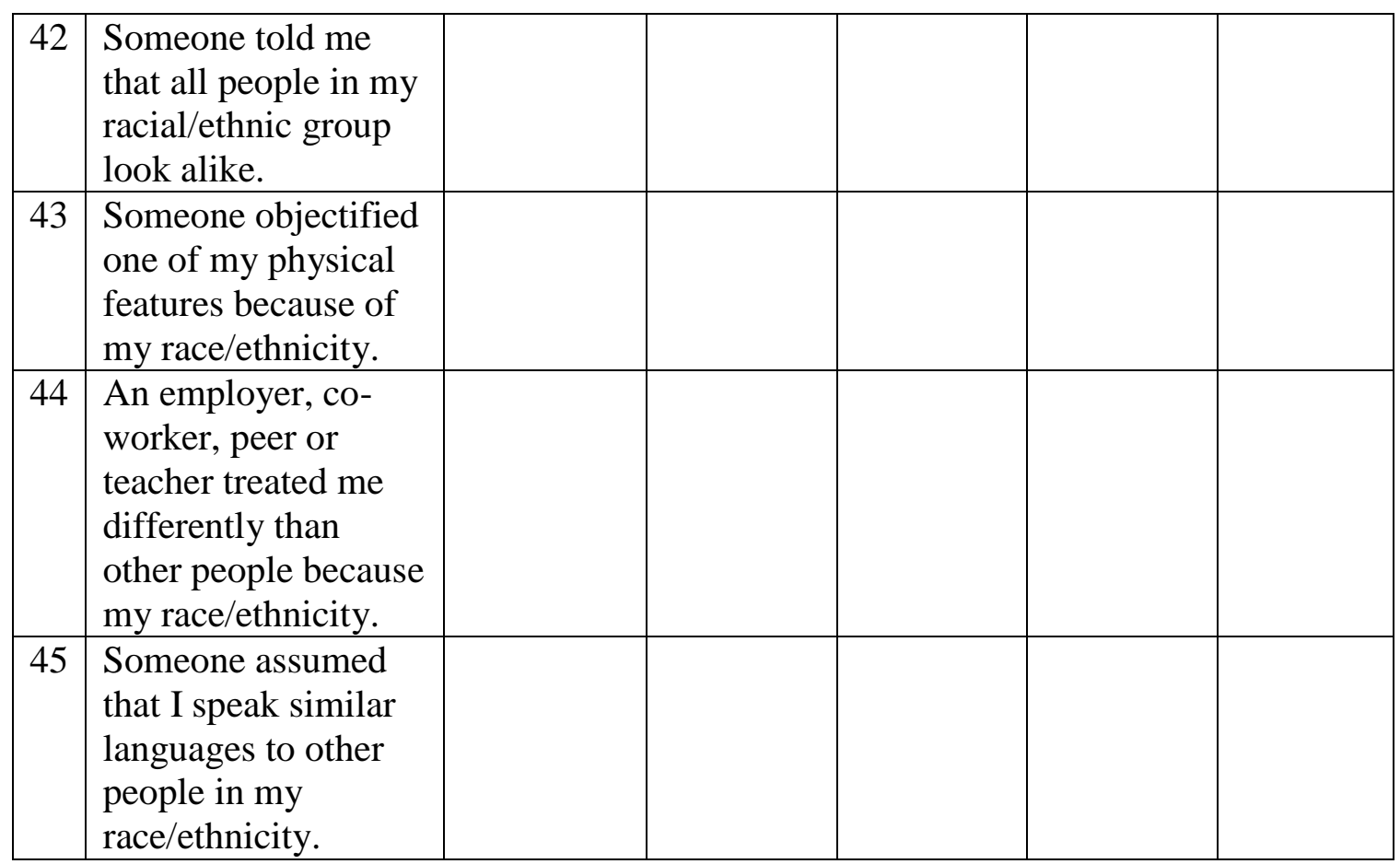

\section{Part 2: Demographic Information}

Instructions: Please answer the following questions. Mark with an $\mathrm{X}$ the box that applies to you.

1. Gender:

2. Age in years:

Male----

$18-20----$

24-26----

3. Marital status:

beyond----

Single----

Divorced----

Other------------

4. Monthly Income:

5. Residence:
\$50-200----

\$351-500----

Other----
Female----

21-23----

26 and

Married----

Separated----

\$201-350----

\$501-650----

In town----

Outside town- 
6. Housing:

Owned by you or your parents but with mortgage or loan---

Owned by you or your parents without mortgage or loan----

Rented----

7. Employment type:

Other---------------------------------

Employed for a private party or business----

Employed for a public or government institution----

Self-employed----

Out of work and looking for work----

A homemaker----

Occasionally employed----

Other-

8. How do you identify by your race/ethnicity?

Indigenous----

Mestizo----

Mulato----

Afro Ecuadorian----

Other

9. Languages you speak

Spanish----

Indigenous language---

Other-------------------------------

10. Languages you write

Spanish----

Other (s) specify

11. Languages spoken by your parents

Spanish----

Other (s) speficy

12. Parents level of education:

Primary completed----

Secondary completed----

University completed with a degree----

Other-------------------------------- 


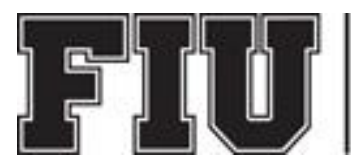

\section{FLORIDA \\ INTERNATIONAL UNIVERSITY}

\section{ROSENBERG SELF-ESTEEM SCALE}

Instructions: below is a list of statements dealing with our general feelings about yourself. If you strongly agree, mark SA. If you agree with the statement, circle A. If you disagree, circle D. If you strongly disagree, circle SD. Please read the following statements and mark the box that corresponds to your opinion with an (X).

\begin{tabular}{|l|l|c|c|c|c|}
\hline \multicolumn{1}{|c|}{ Items } & $\begin{array}{c}\text { Strongly } \\
\text { Agree } \\
\text { SA } \\
\mathbf{3}\end{array}$ & $\begin{array}{c}\text { Agree } \\
\text { A }\end{array}$ & $\begin{array}{c}\text { Disagree } \\
\text { D }\end{array}$ & $\begin{array}{c}\text { Strongly } \\
\text { disagree } \\
\text { SD } \\
\text { 0 }\end{array}$ \\
\hline $\mathbf{1}$ & $\begin{array}{l}\text { I feel that I am a person of worth, at } \\
\text { least on an equal plane with others. }\end{array}$ & & & & \\
\hline $\mathbf{2}$ & $\begin{array}{l}\text { I feel that I have a number of good } \\
\text { qualities. }\end{array}$ & & & & \\
\hline $\mathbf{3}$ & $\begin{array}{l}\text { All in all, I am inclined to feel that I } \\
\text { am a failure. }\end{array}$ & & & & \\
\hline $\mathbf{4}$ & $\begin{array}{l}\text { I am able to do things as well as most } \\
\text { other people. }\end{array}$ & & & & \\
\hline $\mathbf{5}$ & $\begin{array}{l}\text { I feel I do not have much to be proud } \\
\text { of. }\end{array}$ & & & & \\
\hline $\mathbf{6}$ & $\begin{array}{l}\text { I take a positive attitude toward } \\
\text { myself. }\end{array}$ & & & & \\
\hline $\mathbf{7}$ & Overall, I am satisfied with myself. & & & & \\
\hline $\mathbf{8}$ & $\begin{array}{l}\text { I wish I could have more respect for } \\
\text { myself. }\end{array}$ & & & & \\
\hline $\mathbf{9}$ & I certainly feel useless at times & & & & \\
\hline $\mathbf{1 0}$ & At times, I think I am no good at all. & & & & \\
\hline
\end{tabular}




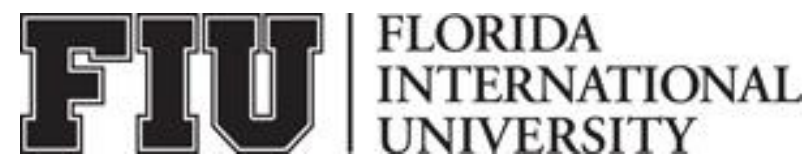

\section{ESCALA DE AUTOESTIMA DE ROSENBERG}

Instrucciones: a continuación usted tiene una lista de afirmaciones referentes a los sentimientos o pensamientos que tiene sobre usted. Si usted está completamente de acuerdo marque CA. Si está de acuerdo A. Si está en desacuerdo D y si está completamente en desacuerdo marque $\mathrm{CD}$. Por favor, lea las afirmaciones y marque con una $(\mathrm{X})$ el casillero que corresponda a su opinión.

\begin{tabular}{|c|c|c|c|c|c|}
\hline & Items & $\begin{array}{l}\text { Completamente } \\
\text { de acuerdo }\end{array}$ & $\begin{array}{c}\text { De } \\
\text { acuerdo }\end{array}$ & $\begin{array}{c}\begin{array}{c}\text { En } \\
\text { desacuerdo }\end{array} \\
\end{array}$ & $\begin{array}{l}\text { Completamente } \\
\text { en desacuerdo }\end{array}$ \\
\hline 1 & $\begin{array}{l}\text { Siento que soy una persona digna de } \\
\text { aprecio, al menos en igual medida } \\
\text { que los demás. }\end{array}$ & & & & \\
\hline 2 & $\begin{array}{l}\text { Creo que tengo un buen número de } \\
\text { cualidades }\end{array}$ & & & & \\
\hline 3 & $\begin{array}{l}\text { En general, me inclino a pensar que } \\
\text { soy un/una fracasado/a }\end{array}$ & & & & \\
\hline 4 & $\begin{array}{c}\text { Soy capaz de hacer las cosas tan bien } \\
\text { como la mayoría de la gente }\end{array}$ & & & & \\
\hline 5 & $\begin{array}{l}\text { Siento que no tengo muchos } \\
\text { motivos para sentirme orgulloso/a } \\
\text { de mí. }\end{array}$ & & & & \\
\hline 6 & $\begin{array}{l}\text { Tengo una actitud positiva hacia mí } \\
\text { mismo/a }\end{array}$ & & & & \\
\hline 7 & $\begin{array}{l}\text { En general, estoy satisfecho/a } \\
\text { conmigo mismo/a. }\end{array}$ & & & & \\
\hline 8 & $\begin{array}{l}\text { Desearía valorarme más a mí } \\
\text { mismo/a }\end{array}$ & & & & \\
\hline 9 & $\begin{array}{l}\text { A veces me siento verdaderamente } \\
\text { inútil }\end{array}$ & & & & \\
\hline 10 & $\begin{array}{l}\text { A veces pienso que no soy bueno/a } \\
\text { para nada }\end{array}$ & & & & \\
\hline
\end{tabular}




\section{APPENDIX G}

\section{GENERAL SELF-EFFICACY SCALE}

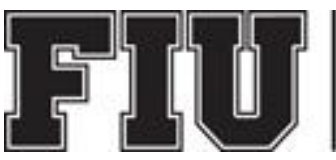

\section{FLORIDA}

INTERNATIONAL

UNIVERSITY

\section{GENERAL SELF-EFFICACY SCALE}

Instructions: below is a list of statements dealing with emotion, optimism, and work satisfaction.Please mark the option that best describes your opinion. Mark exactly true 4; moderately true 3 ; moderately untrue 2 ; not at all true 1 . Please read the statements and mark the box that corresponds to your opinion with an $(\mathrm{X})$.

\begin{tabular}{|c|c|c|c|c|c|}
\hline & Items & $\begin{array}{c}\text { Exactly true } \\
4\end{array}$ & $\begin{array}{c}\text { Moderately } \\
\text { true } \\
3\end{array}$ & $\begin{array}{c}\text { Moderately } \\
\text { untrue } \\
2\end{array}$ & $\begin{array}{c}\text { Not at all } \\
\text { true } \\
1\end{array}$ \\
\hline 1 & $\begin{array}{l}\text { I can always manage to solve } \\
\text { difficult problems if I try hard } \\
\text { enough }\end{array}$ & & & & \\
\hline 2 & $\begin{array}{l}\text { If someone opposes me, I can find } \\
\text { the means and ways to get what I } \\
\text { want }\end{array}$ & & & & \\
\hline 3 & $\begin{array}{l}\text { It is easy for me to stick to my aims } \\
\text { and accomplish my goals. }\end{array}$ & & & & \\
\hline 4 & $\begin{array}{l}\text { I am confident that I could deal } \\
\text { efficiently with unexpected events. }\end{array}$ & & & & \\
\hline 5 & $\begin{array}{l}\text { Thanks to my resourcefulness, I } \\
\text { know how to handle unforeseen } \\
\text { situations. }\end{array}$ & & & & \\
\hline 6 & $\begin{array}{l}\text { I can solve most problems if I invest } \\
\text { the necessary effort. }\end{array}$ & & & & \\
\hline 7 & $\begin{array}{l}\text { I can remain calm when facing } \\
\text { difficulties because I can rely on my } \\
\text { coping abilities. }\end{array}$ & & & & \\
\hline 8 & $\begin{array}{l}\text { When I am confronted with a } \\
\text { problem, I can usually find several } \\
\text { solutions }\end{array}$ & & & & \\
\hline 9 & $\begin{array}{l}\text { If I am in trouble, I can usually think } \\
\text { of a solution }\end{array}$ & & & & \\
\hline 10 & $\begin{array}{l}\text { I can usually handle whatever comes } \\
\text { my way. }\end{array}$ & & & & \\
\hline
\end{tabular}




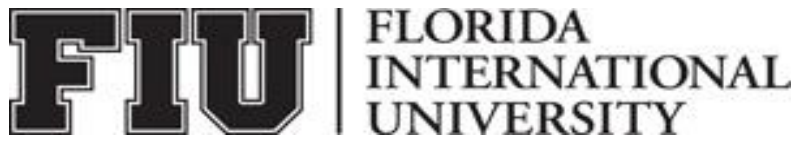

\section{ESCALA DE AUTOEFICACIA GENERAL}

Instrucciones: a continuación, usted tiene una lista de afirmaciones referentes a emociones, optimismo y satisfacción laboral. Si usted considera el enunciado verdadero marque 4; moderadamente verdadero 3; moderadamente no verdadero 2 ; no verdadero 1. Marque sus respuestas con una (X) en el casillero que corresponda.

\begin{tabular}{|c|c|c|c|c|c|}
\hline & Items & $\begin{array}{c}\text { Verdadero } \\
4\end{array}$ & $\begin{array}{l}\text { Moderadamente } \\
\text { verdadero } \\
\mathbf{3}\end{array}$ & $\begin{array}{l}\text { Moderadamente } \\
\text { no verdadero } \\
2\end{array}$ & $\begin{array}{c}\text { No } \\
\text { verdadero } \\
1\end{array}$ \\
\hline 1 & $\begin{array}{l}\text { Puedo resolver problemas } \\
\text { difíciles si hago el esfuerzo } \\
\text { necesario. }\end{array}$ & & & & \\
\hline 2 & $\begin{array}{l}\text { Aunque alguien se me oponga, } \\
\text { puedo encontrar los medios y la } \\
\text { forma de obtener lo que quiero. }\end{array}$ & & & & \\
\hline 3 & $\begin{array}{l}\text { Me resulta fácil enfocarme en los } \\
\text { fines que persogo y lograr mis } \\
\text { objetivos. }\end{array}$ & & & & \\
\hline 4 & $\begin{array}{l}\text { Tengo confianza en que podría } \\
\text { manejar eficazmente situaciones } \\
\text { imprevistas }\end{array}$ & & & & \\
\hline 5 & $\begin{array}{l}\text { Gracias a mis capacidades y } \\
\text { recursos, se cómo manejar } \\
\text { situaciones inesperadas. }\end{array}$ & & & & \\
\hline 6 & $\begin{array}{l}\text { Puedo resolver la mayoría de mis } \\
\text { dificultades si me esfuerzo lo } \\
\text { suficiente. }\end{array}$ & & & & \\
\hline 7 & $\begin{array}{l}\text { Puedo mantener la calma cuando } \\
\text { me enfrento a dificultades porque } \\
\text { confío en mis habilidades para } \\
\text { afrontarlas. }\end{array}$ & & & & \\
\hline 8 & $\begin{array}{l}\text { Cuando me enfrento a un } \\
\text { problema, generalmente puedo } \\
\text { encontrar varias soluciones } \\
\text { alternativas. }\end{array}$ & & & & \\
\hline 9 & $\begin{array}{l}\text { Si tengo problemas, generalmente } \\
\text { se me ocurre una solución. }\end{array}$ & & & & \\
\hline 10 & $\begin{array}{l}\text { Por lo general puedo manejar } \\
\text { cualquier situación que se me } \\
\text { presente. }\end{array}$ & & & & \\
\hline
\end{tabular}




\section{APPENDIX H}

\section{RACIAL MICROAGGRESSIONS INTERVIEW PROTOCOL}

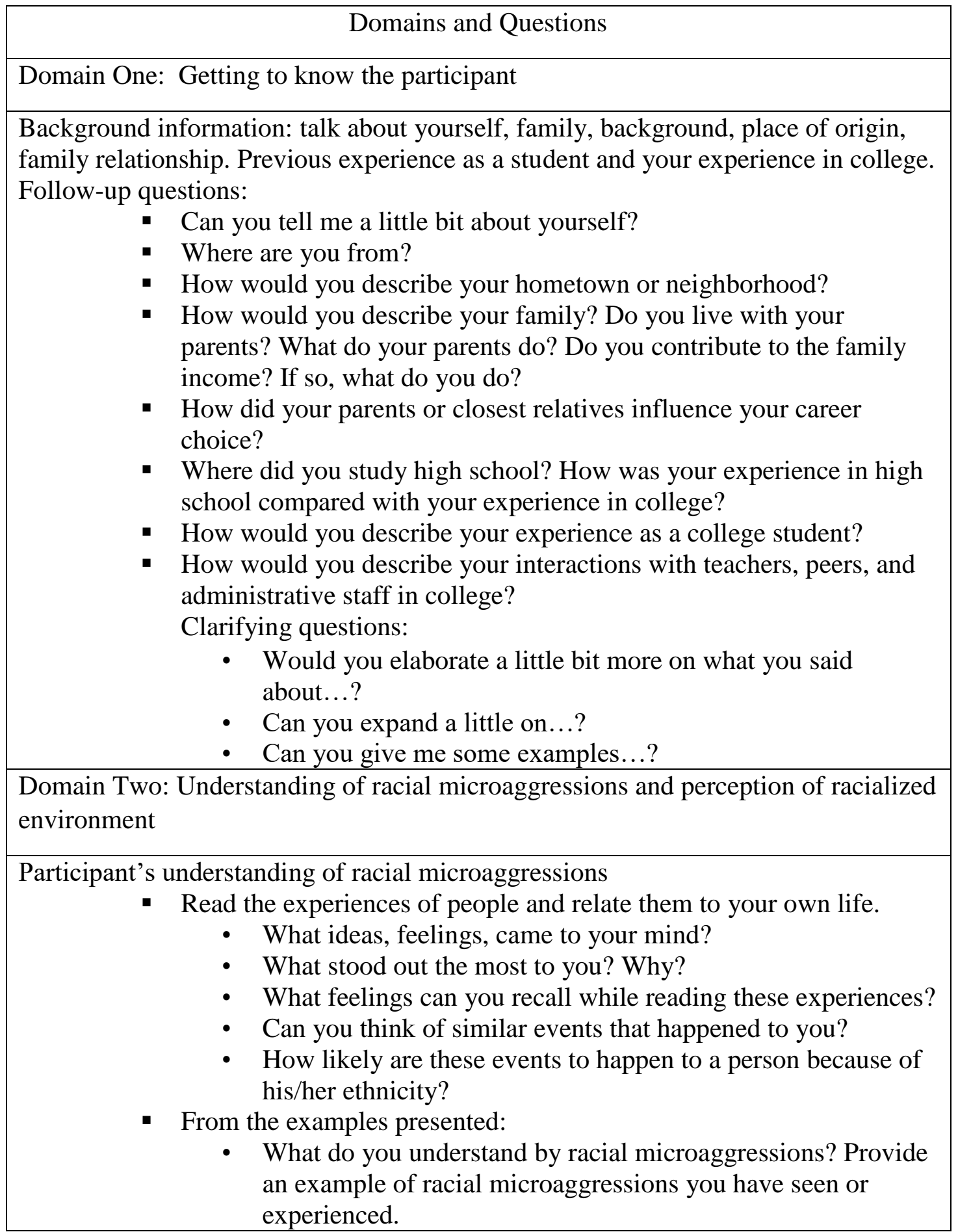




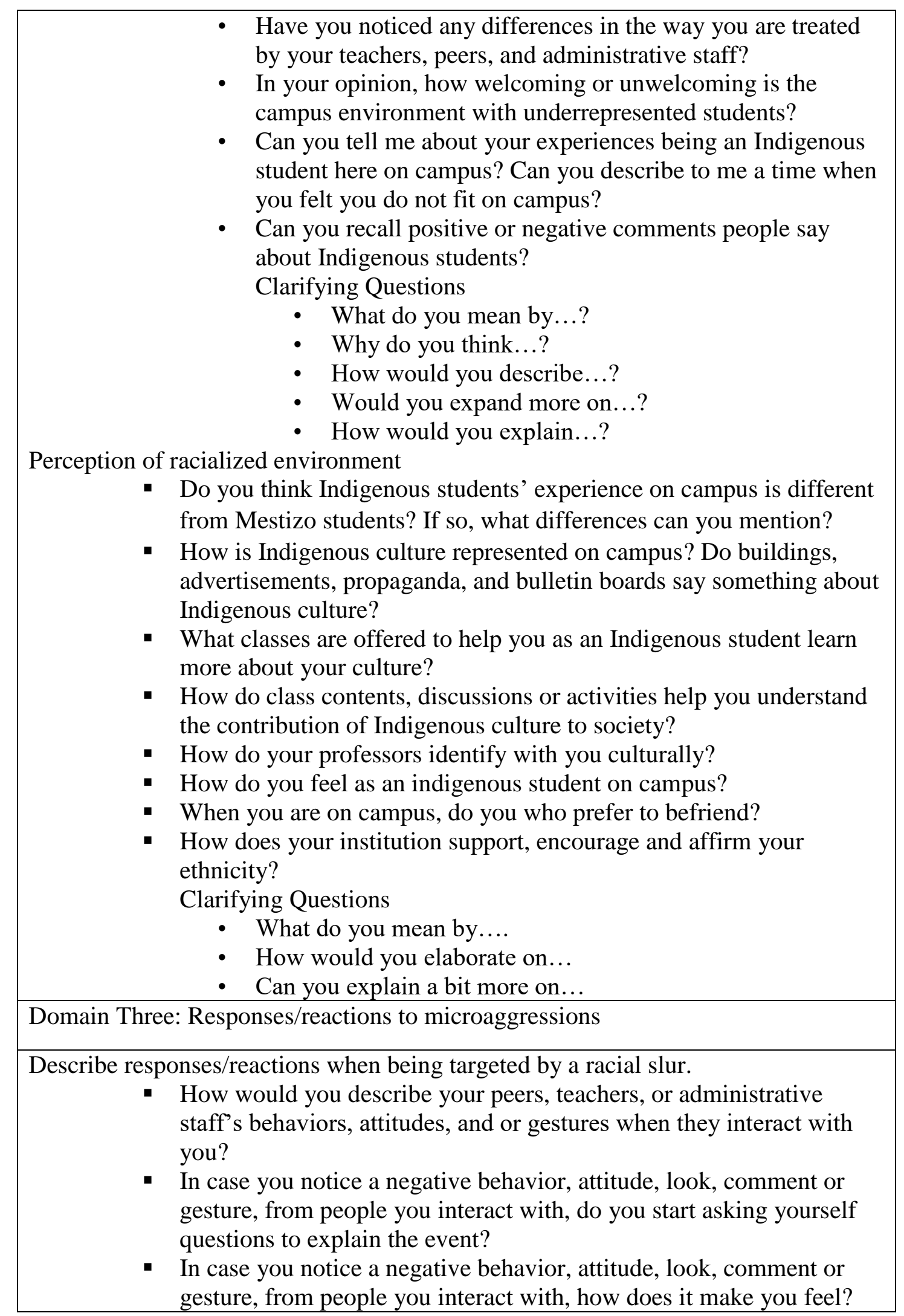


- In case you notice a negative behavior, attitude, look, comment or gesture, from people you interact with, how do you respond or react? Don't you respond? Do you respond verbally? Do you respond physically? Do you ignore the person's action? Do you feel depressed or stressed? Do you feel angry?

Clarifying Questions

- Why do you think you feel...?

- Why do you think...?

- Why do you feel...?

- Can you add more details on...?

\section{Domain Four: Coping with racial microaggressions}

Description of how participants address microaggressions

- When you think you have been mistreated because of your ethnicity, do you ever talk about the incident with your peers, teachers, parents?

- When you think you have been mistreated because of your race, do you seek for peer who have experienced the same issues? Do you look for help from experts like psychologists or counselors?

- When you think you have been mistreated because of your race, do you try to relief your stress by exercising, going shopping, reading, spending time with friends?

- When you think you have been mistreated because of your race, do you oversleep, procrastinate, eat unhealthy, drink alcohol or any other negative behavior?

- To what extent do you think Indigenous students create spaces that help them navigate campus life?

- To what extent do you seek a sense of family or intimacy with your Indigenous counterparts?

- How does your knowledge about your Indigenous culture help you navigate your college experience?

- Do you think Indigenous students have created a sense of community on campus where they can discuss issues that affect them?

- Do you ever use your Quichua language as a tool to show cultural resistance?

- How often do you gather with other Indigenous partners to cultivate a sense of Indigenous community?

- Clarifying Questions

- Is there something else you can tell?

- Would you like to add some more on what you just said? 
PROTOCOLO DE ENTREVISTA PARA MICROAGRESIONES RACIALES

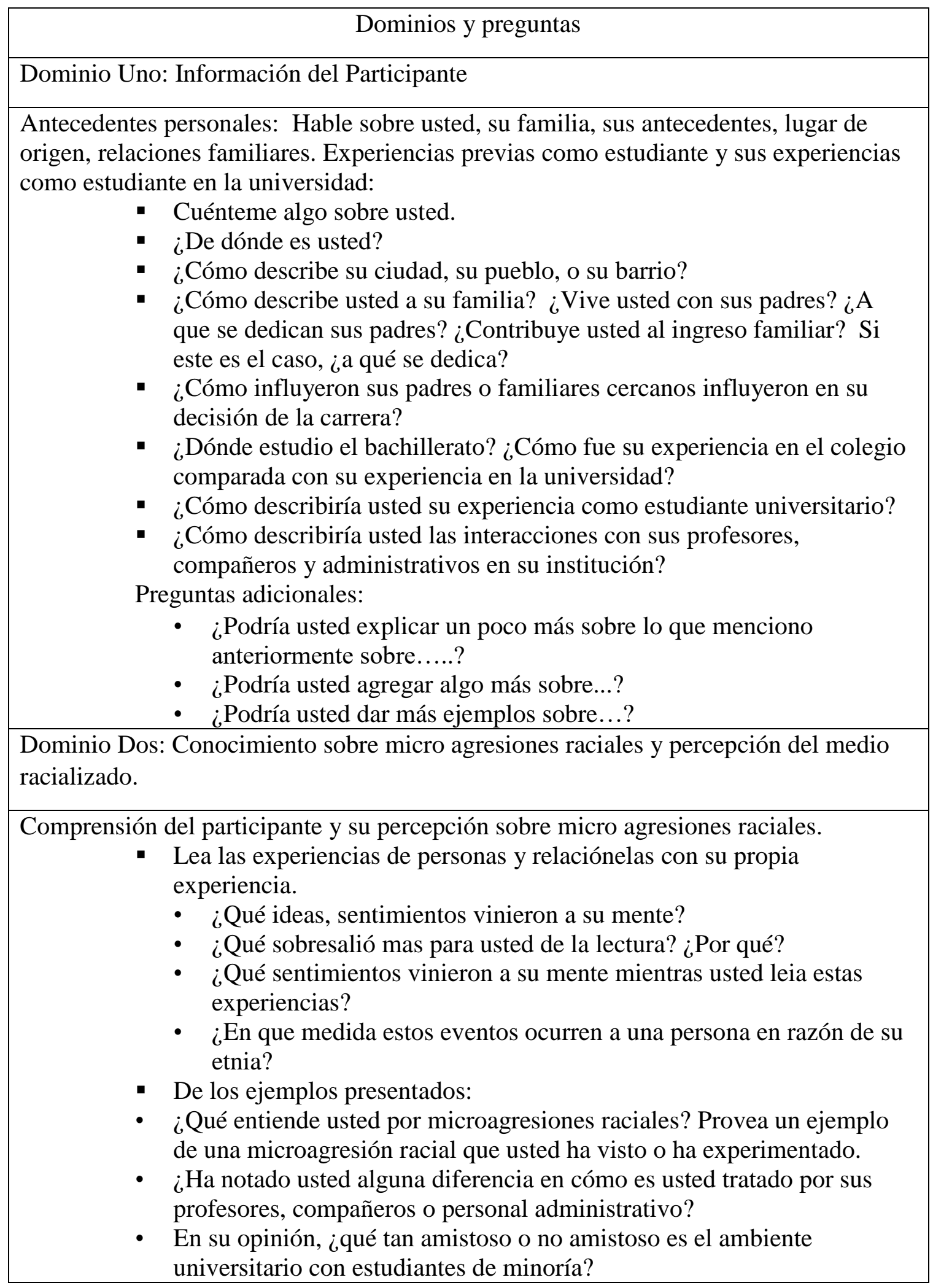







- En el caso que usted note un comportamiento, actitud, comentario o gesto negativo en las personas con quienes usted interactúa, ¿cómo responde usted o cómo reacciona?, ¿usted no responde?, ¿responde usted verbalmente?, ¿responde usted físicamente?, ¿ignora usted la acción de la persona?, ¿se siente usted deprimido o estresado?, ¿se siente usted enojado?

Preguntas adicionales:

- ¿Por qué piensa usted que usted siente...?

- ¿Por qué cree usted...?

- ¿Puede usted añadir más detalles sobre...?

\section{Dominio Cuatro: Manejo de las micro agresiones}

Descripción de como los participantes manejan las micro agresiones

- Cuando usted piensa que ha sido maltratado en relación a su raza, ¿alguna vez habla usted sobre el incidente con sus compañeros, profesores, padres?

- Cuando usted piensa que ha sido maltratado en relación a su raza, ¿busca usted a algún amigo o compañero que ha tenido la misma experiencia?, ¿busca usted la ayuda de expertos tales como psicólogos o consejeros?

- Cuando usted piensa que ha sido maltratado en relación a su raza, ¿trata usted de disminuir su estrés ejercitándose, yendo de compras, leyendo, pasando su tiempo con amigos?

- Cuando usted piensa que ha sido maltratado en relación a su raza, ¿elije usted dormir más, postergar sus actividades, comer comida no saludable, beber alcohol o cualquier otra conducta negativa?

- ¿En qué medida cree usted que los estudiantes Indígenas crean espacios en el campus que les ayudan a navegar la vida universitaria?

- ¿En qué medida busca usted un sentido de familiaridad o intimidad con sus compañeros Indígenas?

- ¿Cómo su conocimiento sobre la cultura Indígena le ayuda a navegar su experiencia en la universidad?

- ¿Cree usted que los estudiantes Indígenas han creado un sentido de comunidad en el campus donde ellos puedan discutir sobre aspectos que los afectan?

- ¿Alguna vez usa usted su idioma Quichua como un instrumento para mostrar resistencia cultural?

- ¿Con que frecuencia se reúne usted con otros compañeros Indígenas para cultivar un sentido de comunidad Indígena?

Preguntas adicionales:

- ¿Hay algo más que usted quiera manifestar?

- ¿Quisiera usted añadir algo más a lo que ya ha dicho? 


\section{APPENDIX I}

\section{ADULT CONSENT FORM TO PARTICIPATE IN THE STUDY}

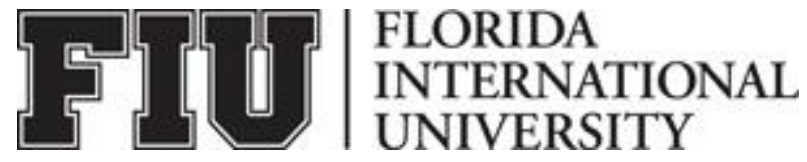

\section{ADULT CONSENT TO PARTICIPATE IN A RESEARCH STUDY}

Racial Microaggressions of Indigenous Undergraduate Students in Higher Education Institution

\section{SUMMARY INFORMATION}

The purpose of the study is to examine the types of microaggressions affecting Indigenous undergraduate students while pursuing a bachelor's degree in a public higher education institution and the associations to their self-esteem and self-efficacy. It also aims to explain students' experiences on how they respond/react and deal with microaggressions.

Things you should know about this study:

- Purpose: The purpose of the study is to examine the types of microaggressions affecting Indigenous undergraduate students while pursuing a bachelor's degree in a public higher education institution and the associations to their self-esteem and self-efficacy. It also aims to explain students' experiences on how they respond/react and deal with microaggressions.

- Procedures: If you choose to participate, you will be asked to answer to a semistructured interview. The interview will take place in two meetings within a week.

- Duration: It will take approximately two hours to complete the interview.

- Risks: Any risk or discomfort from this study is anticipated.

- Benefits: The main benefit to you from this research is to create awareness of racial microaggressions affecting Indigenous students while pursuing a bachelor's degree.

- Alternatives: There are no known alternatives available to you other than not taking part in this study

- Participation: Taking part in this research project is voluntary.

Please carefully read the entire document before agreeing to participate.

\section{PURPOSE OF THE STUDY}

The purpose of this study is to examine the types of microaggressions affecting Indigenous undergraduate students while pursuing a bachelors' degree in a public higher education institution in Ecuador and the associations these microaggressions have on 
their self-esteem and self-efficacy. It also aims to explain the experience of Indigenous students with respect to how they respond/react to and deal with microaggressions.

\section{NUMBER OF STUDY PARTICIPANTS}

If you decide to be in this study, you will be one of ten people in this research study.

\section{DURATION OF THE STUDY}

Your participation will involve approximately two hours.

\section{PROCEDURES}

If you agree to be in the study, we will ask you to do the following things:

1. You will be asked to provide answers to a semistructured interview on your responses/reactions and coping mechanisms when facing microaggressions.

2. You will answer the questions in the interview in two interview meetings. Both interviews will take place within a week. The first interview will include background information about yourself your family, your place of origin, your previous and school experience, the nature of your relationships with teachers and peers, how respected, visible and supported you feel in your institution. The second interview will ask for your awareness regarding microaggressions, perceptions of racialized environment, your responses/reactions to microaggressions, and how you cope with racial hostilities.

3. Your interview will be audio-recorded for further transcription. Any reference to your personal information will be referenced during transcription. Once transcriptions are completed, the recording will be deleted.

\section{RISKS AND/OR DISCOMFORTS}

There are no foreseeable risks for your participation in this research study. However, the study may imply the following implications for you: reflecting on topics related to race may generate some discomfort for the participant. This risk is minimal. If at some point in the interview you consider you do not want to continue, you have the right to withdraw from this study. Withdrawing the study does not imply any penalty. Please do not hesitate to express any adverse reaction you may experience throughout the interview.

\section{BENEFITS}

The study has the following possible benefits to you: it is hoped that your participation will help you to reflect about racial issues that affect you as an Indigenous student. Another benefit is that your reflections will increase awareness to identify racial hostilities in the environment where you develop your activities. We believe that by creating awareness, educational institutions can take action to guarantee a safe and healthy educational environment to all students.

\section{ALTERNATIVES}

There are no known alternatives available to you other than not taking part in this study Any significant new findings developed during the course of the research which may relate to your willingness to continue participation will be provided to you. 


\section{CONFIDENTIALITY}

The records of this study will be kept private and will be protected to the fullest extent provided by law. In any sort of report we might publish, we will not include any information that will make it possible to identify you. Research records will be stored securely, and only the researcher will have access to the records. However, your records may be inspected by authorized University or other agents who will also keep the information confidential.

\section{COMPENSATION \& COSTS}

There are no costs to you for participating in this study.

\section{RIGHT TO DECLINE OR WITHDRAW}

Your participation in this study is voluntary. You are free to participate in the study or withdraw your consent at any time during the study. You will not lose any benefits if you decide not to participate or if you quit the study early. The investigator reserves the right to remove you without your consent at such time that they feel it is in the best interest.

\section{RESEARCHER CONTACT INFORMATION}

If you have any questions about the purpose, procedures, or any other issues relating to this research study you may contact Adriana Cundar Ruano at $11200 \mathrm{SW} 8^{\text {th }}$ Street, ZEB 232, 305-988-9221 or 593-032-960-9673, acund003@ fiu.edu.

\section{IRB CONTACT INFORMATION}

If you would like to talk with someone about your rights of being a subject in this research study or about ethical issues with this research study, you may contact the FIU Office of Research Integrity by phone at 305-348-2494 or by email at ori@fiu.edu.

\section{PARTICIPANT AGREEMENT}

I have read the information in this consent form and agree to participate in this study. I have had a chance to ask any questions I have about this study, and they have been answered for me. I understand that I will be given a copy of this form for my records.

Signature of Participant

Date

Printed Name of Participant

Signature of Person Obtaining Consent

Date 


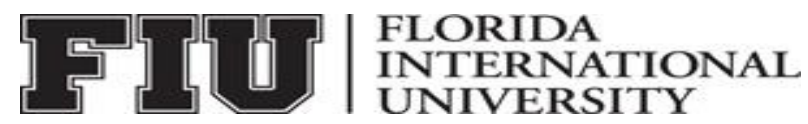

\section{CONSENTIMIENTO DEL ADULTO PARA PARTICIPAR EN UN ESTUDIO DE INVESTIGACION}

Microagresiones Raciales en Estudiantes Indígenas de Pregrado en una Institución de Ecuatoriana de Educación Superior

\section{RESUMEN DE LA INFORMACION}

El propósito de este estudio es examinar los tipos de microagresiones que afectan a estudiantes Indígenas de pregrado mientras realizan sus estudios en una institución pública de educación superior y las asociaciones existentes entre las microagresiones y la autoestima y auto eficiencia de los estudiantes. El estudio también se propone explicar las experiencias de los estudiantes sobre sus respuestas/reacciones y como los estudiantes manejan las microagresiones.

Lo que debe saber usted de este estudio:

- Propósito: El propósito de este estudio es examinar los tipos de microagresiones que afectan a estudiantes Indígenas de pregrado mientras realizan sus estudios en una institución pública de educación superior y las asociaciones existentes entre las microagresiones y la autoestima y auto eficiencia de los estudiantes. El estudio también se propone explicar las experiencias de los estudiantes sobre sus respuestas/reacciones y como los estudiantes manejan las microagresiones.

- Procedimientos: si usted elije participar, se le requerirá responder a una entrevista semiestructurada. La entrevista se llevará a cabo en dos reuniones en el lapso de una semana.

- Duración: para completar la entrevista, se requerirá aproximadamente dos horas.

- Riesgos: ningún riesgo o dificultad se prevén en este estudio.

- Beneficios: el mayor beneficio para usted en relación a este estudio es crear conciencia sobre las microagresiones raciales que afectan a los estudiantes Indígenas mientras cursan su programa de pregrado.

- Alternativas: no existen alternativas para usted a no ser que usted decida no tomar parte en este estudio

- Participación: su participación en este estudio es voluntaria.

Por favor, lea detenidamente este documento antes de consentir su participación.

\section{PROPOSITO DEL ESTUDIO}

El propósito de este estudio es examinar los tipos de microagresiones que afectan a estudiantes Indígenas de pregrado mientras realizan sus estudios en una institución pública de educación superior y las asociaciones existentes entre las microagresiones y la autoestima y auto eficiencia de los estudiantes. El estudio también se propone explicar las experiencias de los estudiantes sobre sus respuestas/reacciones y como los estudiantes manejan las microagresiones. 


\section{NUMERO DE PARTICIPANTES}

Si usted decide participar en este estudio, usted será uno de diez participantes en este estudio de investigación.

\section{DURACION DEL ESTUDIO}

Su participación se llevará a cabo en aproximadamente dos horas.

\section{PROCEDIMIENTOS}

Si usted está de acuerdo en participar en este estudio, se le requerirá realizar las siguientes tareas:

1. Se le pedirá proveer respuestas a una entrevista semiestructurada sobre sus respuestas/reacciones y los mecanismos que usted usa para hacer frente a las microagresiones.

2. Usted responderá a preguntas previstas en la entrevista en dos sesiones. Las dos sesiones se llevarán a cabo en el lapso de una semana. La primera sesión le requerirá proveer información personal, sobre su familia, su lugar de origen, su experiencia previa en instituciones educativas, detalles sobre sus relaciones con profesores, compañeros, cuan respetado, visible y apoyado se siente usted en su institución. En la segunda sesión se le requerirá contestar sobre su conocimiento sobre microagresiones, sus percepciones en relación a un medio ambiente racializado, sus respuestas frente a las microagresiones y como usted maneja las hostilidades raciales.

3. Su entrevista será grabada con propósitos de transcripción. Ninguna referencia a su información personal será revelada durante la transcripción. Una vez las transcripciones hayan sido completadas, la grabación será borrada.

\section{RIESGOS Y/O DESCONOCIMIENTOS}

Ningún riesgo previsible está asociado a su participación en este estudio de investigación. Sin embargo, el estudio puede implicar los siguientes efectos para usted: reflexionar sobre temas relacionados con la raza puede generar cierta incomodidad para el participante. Este riesgo es mínimo. En caso de que en algún punto de la entrevista usted sienta que no desea continuar, usted tiene derecho a retirarse del estudio. Retirarse del estudio no implica ninguna sanción. Por favor, no dude en expresar cualquier reacción adversa durante la entrevista.

\section{BENEFICIOS}

El estudio implica los siguientes beneficios para usted: se espera que su participación lo ayude a reflexionar sobre los problemas raciales que lo afectan como estudiante Indígena. Otro beneficio es que sus reflexiones pueden aumentar su conciencia para identificar las hostilidades raciales presentes en el entorno donde desarrolla sus actividades. Creemos que, al crear conciencia, las instituciones educativas pueden tomar medidas para garantizar un ambiente educativo seguro y saludable para todos los estudiantes

\section{ALTERNATIVAS}

No hay alternativas conocidas disponibles para usted aparte de no participar en este estudio. Se le proporcionará cualquier hallazgo nuevo significativo desarrollado durante 
el curso de la investigación que pueda estar relacionado con su disposición a continuar participando.

\section{CONFIDENCIALIDAD}

Los registros de este estudio se guardarán de manera privada y serán protegidos en la mayor medida prevista por la ley. En cualquier tipo de reporte que se publique, no incluiremos ninguna información que posibilite la identificación del participante. Los registros de investigación se almacenarán de forma segura y solo el investigador tendrá acceso a los mismos. Sin embargo, sus registros pueden ser revisados para fines de auditoría por la Universidad autorizada u otros agentes que estarán sujetos a las mismas disposiciones de confidencialidad.

\section{COMPENSACION Y COSTOS}

There are no costs to you for participating in this study.

\section{DERECHO PARA DECLINACION O RETIRO}

Su participación en este estudio es voluntaria. Usted es libre de participar en el estudio o retirar su consentimiento en cualquier momento durante el mismo. Su retiro o falta de participación no afectará ningún beneficio al que tenga derecho. El investigador se reserva el derecho de eliminarlo sin su consentimiento en el momento en que considere que es lo mejor para usted.

\section{INFORMACION DE CONTACTO DEL INVESTIGADOR}

Si usted tiene preguntas sobre el propósito, procedimientos o cualquier otro aspecto relacionado con este estudio, usted puede contactar a Adriana Cundar Ruano en la siguiente dirección 11200 SW $8^{\text {th }}$ Street, ZEB 232, 305-988-9221 o acund003@ fiu.edu.

\section{INFORMACION DE CONTACTO DEL IRB}

Si desea hablar con alguien sobre sus derechos como participante en este estudio de investigación o sobre cuestiones éticas con este estudio de investigación, puede comunicarse con la Oficina de Integridad de Investigación de la Universidad International de Florida (FIU) por teléfono al 305-348-2494 o por correo electrónico a ori@fiu.edu.

\section{CONVENIO DEL PARTICIPANTE}

He leído la información en este formulario de consentimiento y acepto participar en este estudio. He tenido la oportunidad de hacer cualquier pregunta que tenga sobre este estudio, y me la han respondido. Entiendo que me darán una copia de este formulario para mis registros.

Firma del Participante

Nombre Impreso del Participante

Firma de la persona que obtiene el consentimiento
Fecha

Fecha 
VITA

\section{ADRIANA CUNDAR RUANO}

Born, Quito, Ecuador

B.A., English

Universidad Técnica Particular de Loja

Loja, Ecuador

2003

M.S., University Teaching and Research Universidad Nacional de Chimborazo

Riobamba, Ecuador

2012

M.S., Linguistics Applied to English Learning Universidad Nacional de Chimborazo

Riobamba, Ecuador

2012-2013

Hubert Humphrey Fellowship - Fulbright

Pennsylvania State University

State College, Pennsylvania

2016-2018

Fulbright Scholarship

Florida International University

Miami, Florida

2018-2020

Teaching Assistant

College of Education

Florida International University

Miami, Florida

2020

Doctoral Candidate

Florida International University

\section{PUBLICATIONS AND PRESENTATIONS}

Cundar, A. (2020, July 14). Racial Microaggressions of Indigenous Students in Ecuador. Presentation at the Global Gateways Teacher Training Institute. Florida International University.

Cundar, A. (2020, June 19-20). Indigenous undergraduate students: Experiences of Racial Microaggressions at a Public Higher Education Institution in Ecuador. [Conference session]. 11 ${ }^{\text {th }}$ American Indian Teacher Education Conference AIITEC. Flagstaff, AZ, United States. (Conference Canceled). 
Mathews, S.; Stanislaus, E.; Cundar, A.; Alberte, J.; Magoulas, A.; Lapaix, G. (2020, April 17-21). Exploring intersectionalities of cultural and civic identities with undergraduate students attending a Hispanic-serving institution. Citizenship and Education in an Era of Displacement and Migration. American Educational Research Association AERA. San Francisco, CA, United States. (Conference Canceled).

Mathews, S.; Alberte, J.; Naranjo, J.; Rosenberg, G.; Lapaix, G.; Stanislaus, E.; Page, A.; Cundar, A.; Pereira, R. (2020, March 22-26). Citizenship and Education in an Era of Displacement and Migration. [Conference session]. Comparative and International Education Society Conference CIES. Miami, Fl, United States. (Conference Canceled).

Cundar, A. (2018, October 21). Culturally responsive teaching to second language students. [Conference session]. TESOL International Association. Miami, FL, United States.

Cundar, A. (2016). Critical Reflections from Peer Observation of Teaching: A meaningful experience for professional development. SATHIRI Journal. 11, 290-299.

Cundar, A. (2015, April 16-20). Peer Observation and Reflection of Teaching. Strategies to Enhance Professional Development, Cooperation and Collegiality. [Conference session]. $9^{\text {th }}$ International Conference on Linguistics. José A. Portuondo Literature and Linguistics Institute. Habana-Cuba. 